IN-SITU SPECTROSCOPY ANDNANOSCALE ELECTRONICS IN SUPERCONDUCTOR-TOPOLOGICAL INSULATOR HYBRID DEVICES

A combined thin film growth end quantum transport study 


\section{IN-SITU SPECTROSCOPY AND NANOSCALE ELECTRONICS IN SUPERCONDUCTOR-TOPOLOGICAL INSULATOR HYBRID DEVICES}

A combined thin film growth and quantum transport study 


\section{Graduation committee}

\section{Chairman and secretary}

prof. dr. H. B. J. Karperien

University of Twente

\section{Supervisors}

prof. dr. ir. A. Brinkman

University of Twente

prof. dr. ir. J. W. M. Hilgenkamp

University of Twente

\section{Members}

prof. dr. Thomas Schäpers

Forschungsinstitut Jülich, Germany

prof. dr. Mark S. Golden

University of Amsterdam

prof. dr. ir. Gertjan Koster

University of Twente

prof. dr. R. M. van der Meer

University of Twente

assoc. prof. dr. ir. Michel de Jong

University of Twente

\section{ice. $\quad$ UNIVERSITY OF TWENTE.}

MESA+

The research described in this dissertation was performed in the quantum transport in matter (QTM), and the interfaces and correlated electron systems (ICE) groups of the MESA ${ }^{+}$Institute for nanotechnology, and the faculty of science and technology of the University of Twente, The Netherlands.

\section{FOM}

This research work was funded by the Dutch Foundation for Fundamental Research on Matter (FOM), which is part of Dutch Organization for Scientific Research (NWO), and by the European Research Council (ERC).

Cover: Atomic resolution image (top) of a $\mathrm{Bi}_{2} \mathrm{Te}_{3}$ film. An angle resolved photoemission spectroscopy image (bottom) of the same $\mathrm{Bi}_{2} \mathrm{Te}_{3}$ film. Designed by Prosper Ngabonziza.

Printed by: Gildeprint drukkerijen, Enschede.

ISBN: 978-90-365-4168-8

DOI: $10.3990 / 1.9789036541688$

(C) P. Ngabonziza, 2016 


\title{
IN-SITU SPECTROSCOPY AND NANOSCALE ELECTRONICS IN SUPERCONDUCTOR-TOPOLOGICAL INSULATOR HYBRID DEVICES
}

A COMBINED THIN FILM GROWTH AND QUANTUM TRANSPORT STUDY

\section{DISSERTATION}

\author{
to obtain \\ the degree of doctor at the University of Twente, \\ on the authority of the rector magnificus, \\ prof. dr. H. Brinksma, \\ on account of the decision of the graduation committee \\ to be publicly defended \\ on Friday $02^{\text {nd }}$ September 2016 at $16: 45$ hrs
}

by

Prosper Ngabonziza

born 09-November-1981

in Huye, Rwanda 
This dissertation has been approved by:

prof. dr. ir. A. Brinkman

prof. dr. ir. J. W. M. Hilgenkamp 


\section{Contents}

1 Combining the material science and the physics of topological insulators $\quad 1$

1.1 Technological motivation . . . . . . . . . . . . . . . . . 2

1.2 Topological insulators . . . . . . . . . . . . . . . . . . . . . . . 4

1.3 This Dissertation: Topological insulator thin films and topological Josephson devices * 10

2 Growth and surface morphology of $\mathrm{Bi}_{2} \mathrm{Te}_{3}$ topological insulator thin films

2.1 Substrate selection and treatment . . . . . . . . . . . . . . . . . 22

2.2 Thin films deposition and characterization . . . . . . . . . . . . . . . 24

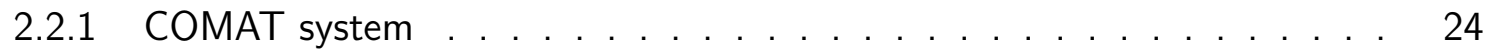

2.2.2 Electron beam evaporation . . . . . . . . . . . . . . . . . . . . . . . . 24

2.2.3 In-situ X-ray photoemission spectroscopy . . . . . . . . . . . . . . . . . 27

2.2 .4 Vacuum suitcase . . . . . . . . . . . . . . . . . . . . . . . . . . . . . . . 28

2.2.5 In-situ angle resolved photoemission spectroscopy . . . . . . . . . . . 30

2.2.6 In-situ scanning tunnelling microscopy . . . . . . . . . . . . . . . . . . . . 31

2.3 Growth dynamics . . . . . . . . . . . . . . . . . . . . . . . . . . . 32

2.3.1 Flux calibration and flux ratio optimization . . . . . . . . . . . . 33

2.3.2 Surface passivation: Two-step growth . . . . . . . . . . . . . . 35

2.3.3 Substrate temperature . . . . . . . . . . . . . . . 35

2.4 Surface morphology of $\mathrm{Bi}_{2} \mathrm{Te}_{3}$ thin films . . . . . . . . . . . . . 37 
3 Magnetoresistance in $\mathrm{Bi}_{2} \mathrm{Te}_{3}$ topological insulator thin films 45

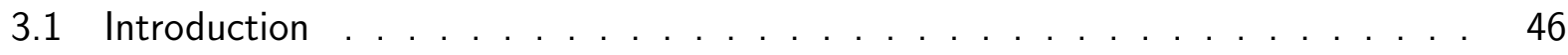

3.2 Magnetotransport in $\mathrm{Bi}_{2} \mathrm{Te}_{3}$ thin films . . . . . . . . . . . . . 46

3.2.1 Weak antilocalization and non-linear Hall effect in $\mathrm{Bi}_{2} \mathrm{Te}_{3}$ thin films . . . 47

3.2.2 Shubnikov-de-Haas oscillations in $\mathrm{Bi}_{2} \mathrm{Te}_{3}$ thin films . . . . . . . . . . . 50

3.2.3 Linear magnetoresistance in $\mathrm{Bi}_{2} \mathrm{Te}_{3}$ thin films . . . . . . . . . . . . . . 54

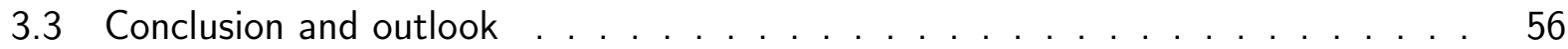

4 In-situ spectroscopy of intrinsic $\mathrm{Bi}_{2} \mathrm{Te}_{3}$ thin films and impact of extrinsic defects 61

4.1 Introduction . . . . . . . . . . . . . . . . . . . 62

4.2 Films growth and characterization . . . . . . . . . . . . . . . 63

4.3 In-situ spectroscopy f . . . . . . . . . . . . . . . . . . . . . 67

4.4 Model calculation of density of states and dispersion relations . . . . . . . . . 71

4.5 Impact of extrinsic defects . . . . . . . . . . . . . . . . . . . . 72

4.6 Conclusion and outlook . . . . . . . . . . . . . . . . . . . . . . 75

5 Gate-tunable transport properties of in-situ capped $\mathrm{Bi}_{2} \mathrm{Te}_{3}$ topological insulator $\begin{array}{ll}\text { thin films } & 81\end{array}$

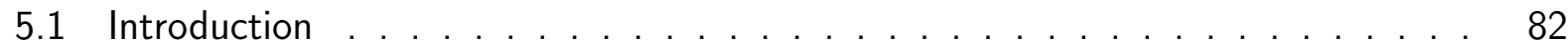

5.2 In-situ surface characterization f . . . . . . . . . . . . . . . 85

5.3 Gate-tunable transport characteristics . . . . . . . . . . . . . . . . . 86

5.3.1 Back-gate dependence of in-situ $\mathrm{Al}_{2} \mathrm{O}_{3}$-capped $\mathrm{Bi}_{2} \mathrm{Te}_{3}$ film . . . . . . . . 89

5.3.2 Back-gate dependence of the in-situ Te-capped $\mathrm{Bi}_{2} \mathrm{Te}_{3}$ film . . . . . . . 92

5.4 Conclusion and outlook . . . . . . . . . . . . . . . . . . . . . . . . . . . 94

6 Combined gate-tunable Josephson supercurrent and normal state transport $\begin{array}{ll}\text { in } \mathrm{Nb}^{-\mathrm{Bi}_{2}} \mathrm{Te}_{3}-\mathrm{Nb} \text { hybrid devices } & 101\end{array}$

6.1 Introduction . . . . . . . . . . . . . . . . . . . 102 
6.2 Thin films quality and Josephson junctions characteristics . . . . . . . . . 103

6.3 Gate dependence of Josephson supercurrent and normal state transport . . . . . 106

6.4 Discussion on unusual gate-dependence of Josephson supercurrent . . . . . . . . 109

6.5 Conclusion and outlook . . . . . . . . . . . . . . . . . . . 111

7 Future directions $\quad 119$

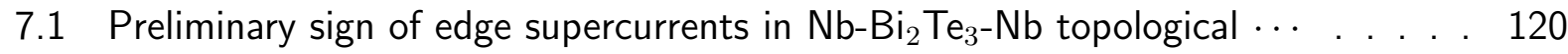

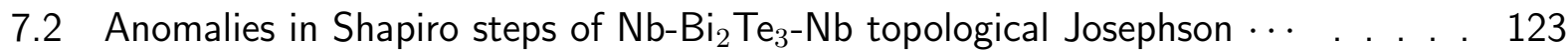

$\begin{array}{ll}\text { Summary } & 131\end{array}$

Samenvatting (Summary in Dutch) 135

$\begin{array}{ll}\text { Acknowledgements } & 139\end{array}$ 

1

\section{Combining the material science and the physics of topological insulators}

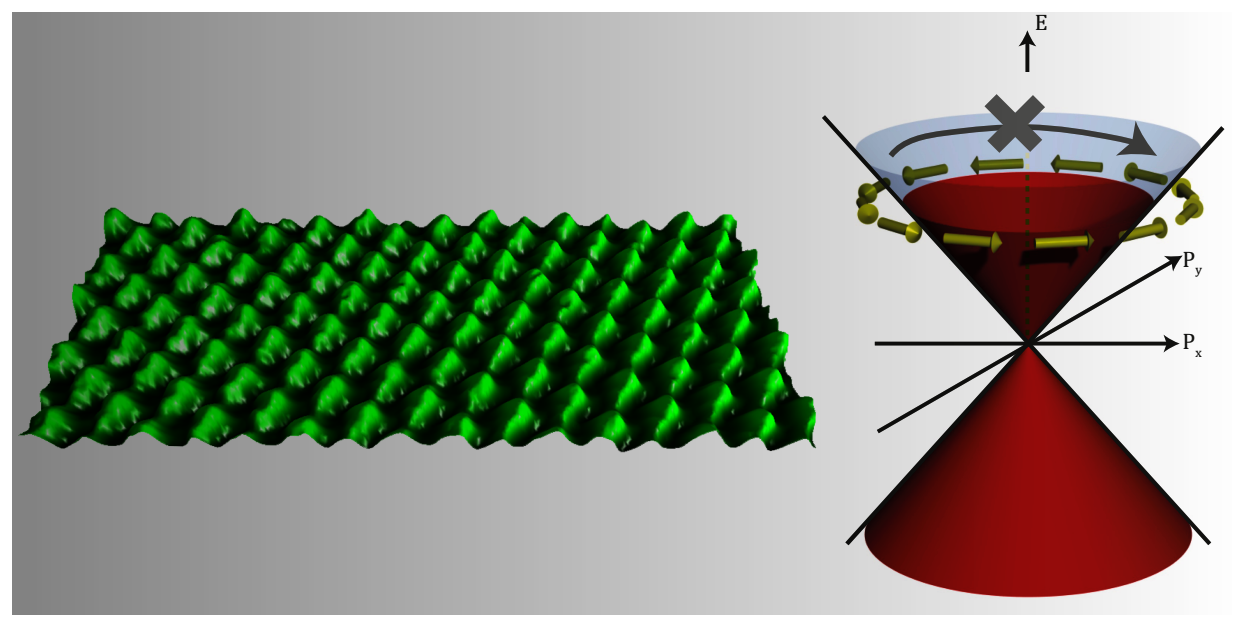

This first chapter introduces the essential concepts of topological insulators that generated enthusiasm in the field of condensed matter physics. In this chapter, we aim not only to present briefly the underling physics of topological materials from a fundamental point of view, but also to explore their potential technological applications. Special attention is paid to the practical necessity of using thin films of topological insulators and the combination of these thin films with s-wave superconductors in hybrid topological Josephson junctions devices. At the end of this chapter, a short overview of the subsequent chapters is also presented. 
Page 2 Chapter 1 . Combining the material science and the physics of topological insulators

\subsection{Technological motivation}

In the field of condensed matter physics, interface and surface physics have become important disciplines. They are studied with the purpose of discovering and designing new materials, mostly emphasising on solids, with the objective of understanding the physics underlying these new materials and their interfaces. These novel materials often exhibit unconventional electronic properties. Due to the advanced miniaturization in nanotechnology, a more clear comprehension of these novel materials and their interfaces at the atomic scale is required. An in-depth grasp of the electronic properties of these materials is extremely useful in delivering lasting impact in broad areas of technological importance, paving the way to numerous practical applications in electronic, spintronic and optoelectronic devices.

The goal is to address existing challenges in present day semiconductor technology. For example, modern semiconductor devices rely on the manipulation of electronic charge (or electrical current) to encode "information". However, to stay competitive, the micro- and nano-electronics industry is following the trajectory defined by a relentless formula, often popularly known as Moore's law, stating that the microprocessors double in power every approximately two years as electronic devices shrink and more logic is packed onto every chip [1]. Yet, Moore's law will run out of momentum sooner or later, since the size of individual bits will eventually approach the quantum limit (dimension of atoms). For this reason and also because the transport of electrons is dissipative (they are scattered by static defects or phonons), research is shifting focus onto another degree of freedom of the electron, known as spin (see Fig. 1.1). The spin of the electron is an intrinsic property of the electron, which can be interpreted as the magnetic moment of a single electron. Still, in order to significantly reduce the power dissipation of these chips due to scattering of electrons, there is a need to increase the crystal quality of semiconductor devices.

In recent years, devices were invented that are capable of manipulating and detecting electron spin. Giant magnetoresistance (GMR) [2, 3] and tunneling magnetoresistance (TMR) [4] devices were engineered to easily measure changes in the electron spin by measuring changes in the resistance of a material under the action of a magnetic field. This marked the beginning of the emerging field of spin-electronics, named "spintronics". While metallic spintronics has already delivered functional systems, for example the use of GMR materials in read heads for highdensity magnetic (data) recording, current research efforts in spintronics are mostly focusing on semiconductor spintronic devices with multiple functionality (for example carrying out processing and data storage on the same chip) [5]. The developments in semiconductor spintronics open the possibility of engineering devices that consume less power and have higher performance 
(a) Electron

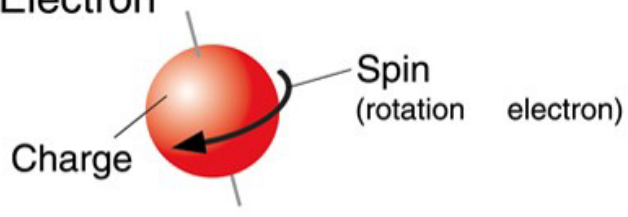

(b) Charge current

(c) Spin current

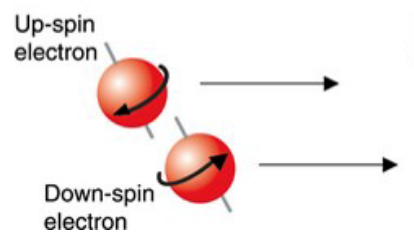

Figure 1.1: Electron's charge and spin. (a) The charge and spin degree of freedoms of an electron. (b) A flow of charge leads to an electric (charge) current. In an ordinary electric current, there is an equal number of up- and down-spin electrons, leading to zero net spin current. (c) To generate a spin current, it requires a clever method of creating a "counter flow" of the up and down spins of electron.

(fast switching) for certain types of computations than is possible with electron-charge-based semiconductor devices. Furthermore, the long term goal of the field of spintronics is to achieve the integration of electronic, optoelectronic and magneto-electronic multifunctionality in a single device, with no external (charge) current needed to manipulate the spin information (up or down) [6]. Such devices will outperform current micro- and nano-electronic systems both in speed and integration density.

To meet this goal, there needs to be a deep understanding of how spins move through the material, of the role of the interfaces between different materials, and how to create and detect spin polarization. One way to test these ideas is by directly injecting an electric current with ferromagnetic contacts, which induces a net spin imbalance in an otherwise nonmagnetic semiconductor [7]. However, experiments indicated that the typical spin polarization is essentially smaller than inside the ferromagnetic spin injector (the achieved spin-polarized electrons were only $\sim 10 \%$ of all flowing electrons) [8]. An interesting solution to circumvent this limitation may be achieved when a new class of recently discovered materials, namely topological insulators (Tls), is used. These materials feature a new electronic state of matter. They behave as ordinary insulators in their interior (bulk), but they are conducting at the surface. Topological surface states have been proposed theoretically as an intrinsic spin filter and spin separator with a spin-polarization of more than $50 \%[9,10]$. Furthermore, because of their exceptionally low electron-phonon coupling, they hold promising application for future room temperature spintronic devices $[11,12]$. 


\subsection{Topological insulators}

A topological insulator $(\mathrm{TI})$ is a material that has an insulating interior (bulk) while its boundaries are conducting. The bulk of topological insulators is characterized by an energy gap in the electronic band structure, whereas the edges or surfaces host non-gapped states which cross the bulk band gap, thus enabling conduction on the boundaries of the material [13]. The surface states of a TI mimic relativistic Dirac electrons [14] because of their linear energy-momentum $E(k)$ relation. The appearance of metallic surface/edge states is due to the strong spin-orbit coupling (SOC) in the material, which causes a band inversion. Figure 1.2 depicts a schematic illustration of the band structure of a normal insulator (see Fig. 1.2 A), of a topological insulator (see Fig. 1.2 C) and an analogy illustrating the concept of topology in the context of electronic band structure (see Fig. 1.2 B). The term "topological" is used because the band structure of TI materials have a different topology compared to normal band insulators. Starting from the band structure of an ordinary insulator, the special topology of the 2D and 3D Tls is realized as a consequence of an inverted band gap of the bulk when the surface of a $\mathrm{Tl}$ is surrounded by a topologically trivial material, such as an ordinary insulator or vacuum. It is the strong SOC that causes this band inversion, and due to the different orbital character of the conduction and valence bands in material with spin-orbit coupling, the final band structure cannot be "deformed" into that of the non-inverted without first closing the band gap at the interface and then reopening it with the reversed sign of the effective band mass [13]. The topology ${ }^{1}$ analogy, from two different topological objects (a sphere and a torus), is drawn below the band structure (see lower panel Fig. 1.2 B). The closing of the band gap in the electronic band structure corresponds to creating a hole in the sphere, which changes its topology since just by simple deformation of the sphere there is no way it can be transformed into a torus without cutting a hole into it. The two objects have different topology [15]. Thus, due to the resemblance with normal insulators on one hand and the topological difference on the other hand, these materials are called topological insulators.

A topological invariant ${ }^{2}$ can be defined in the context of band structures, which enables an identification of the material belonging either to the class of trivial insulators or topological insulators $[14,17]$. It has been shown that the number of times the surface states intersect

\footnotetext{
${ }^{1}$ In mathematics, topology deals with the properties of space that are preserved under continuous deformations, such as stretching and bending, but tearing is not allowed [15]. A sphere is topologically different from a torus since the torus has a hole in it while the sphere does not.

${ }^{2}$ In topology, the term "genus" is used as a topological invariant and it basically gives the number of holes in a three dimensional object. Topological invariants are quantities or properties that are shared by different objects of the same topological "character", but distinct from that of objects belonging to another topological phase [15].
} 
(A)

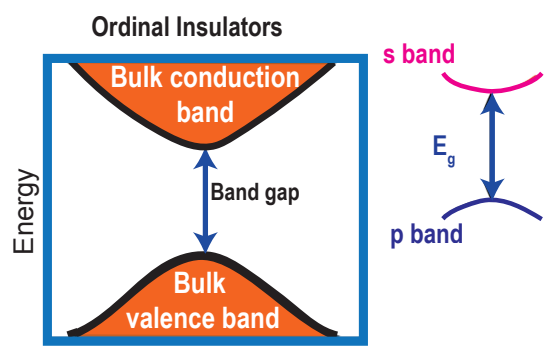

Momentum
(B)

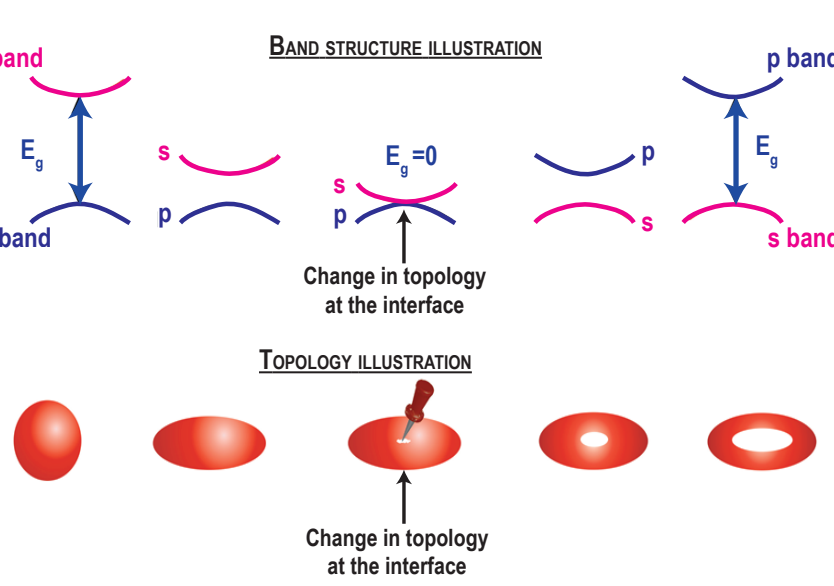

(C)

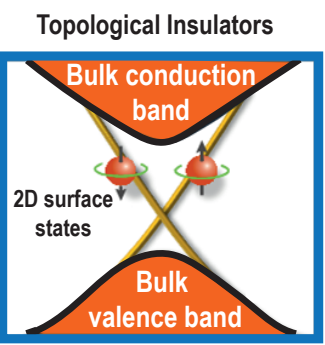

Momentum

Figure 1.2: Topology in condensed matter physics. (A) Drawing of the band structure of a normal insulator with a band gap. (B) Illustration of change in topology in the band structure from an ordinary insulator to a topological insulator. There is a topological change in the band structure at the interface and due to the strong spin orbit coupling in the $\mathrm{TI}$ material: the band order is reversed at the TI side. Similarly, a topological change takes place when coming from a sphere to a torus. (C) Illustrative band structure of ideal three dimensional Tls with 2D surface states with spin up and down crossing from bulk valence band to bulk conduction band.

the Fermi level $\left(\mathrm{E}_{\mathrm{F}}\right)$ is related to the change in the topological invariant $(\Delta \nu)$ when crossing the interface between the TI material and its surroundings trivial insulators $[18,19]$. In the topological insulator context, this is known as the bulk-boundary correspondence. Roughly speaking, the topological invariant $\nu$ is given by [18]:

$$
\nu=N_{K} \bmod 2
$$

where $N_{K}$ is the number of times the surface states intersect the Fermi level along the line in the Brillouin Zone (BZ) between two Kramers degenerate points [19]; $\nu=0$ for trivial band insulators whereas $\nu=1$ for non-trivial band insulators.

The TI surface states are thus a result of the topology of the bulk band structure. Therefore, they are intrinsic to the $\mathrm{TI}$ phase and robust, i.e, they cannot be removed unless there is a change in the topology. Figure 1.3 (a) and 1.3 (b) show a schematic drawing and an experimental angle resolved photoemission (ARPES) spectrum of the surface states of a TI system, respectively. A special feature of these surface states is that they form the so-called Dirac cone, i.e, the surface carrier energy depends linearly on the momentum. The states are protected by time-reversal symmetry and are thus robust against non-magnetic back-scattering [14, 18, 19]. Furthermore, another key aspect of the surface states is that the electron spin is locked to the direction of 
Page 6 Chapter 1. Combining the material science and the physics of topological insulators
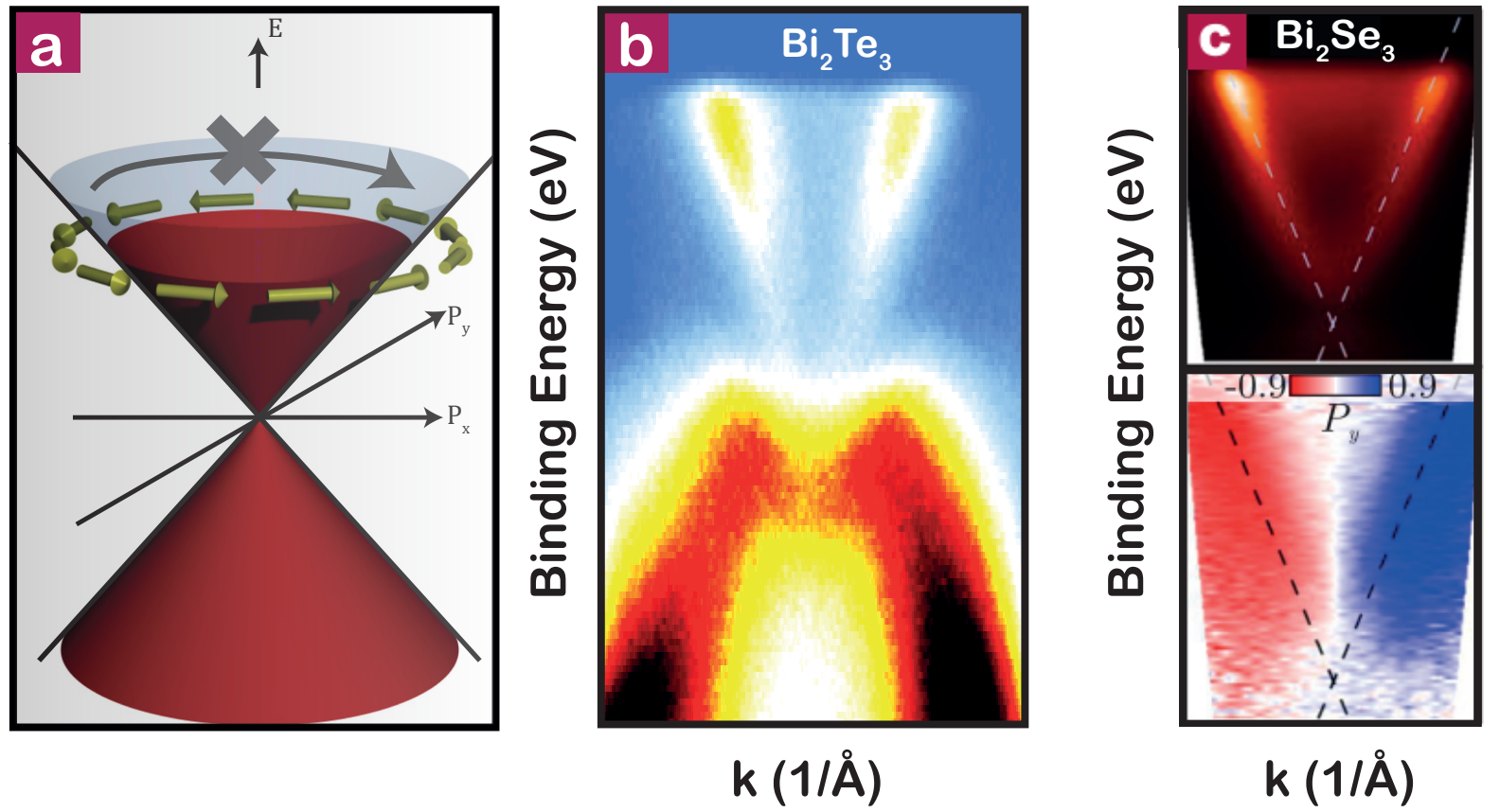

Figure 1.3: Spin helicity in exotic band structure of topological insulators. (a) Helical Dirac cone at zero magnetic field of an ideal TI. Backscattering is not allowed in such a system. (b) Experimental angle-resolved photoemission spectroscopy spectra of the 3D $\mathrm{TI} \mathrm{Bi}_{2} \mathrm{Te}_{3}$. The linear surface bands are clearly resolved. However, the Dirac point is shadowed by bulk valence band states (Fig. is from Ref. [16]). (c) ARPES spectrum of $\mathrm{Bi}_{2} \mathrm{Se}_{3}$ (top) with visible Dirac point and the corresponding (below) spin-polarized spectrum showing the helicity of the surface states. The color scale along $P_{y}$ represents the different spin-polarization (Fig. from Ref. [12]).

motion, as depicted by the spin-polarized ARPES data shown in Fig. 1.3 (c). Electrons moving in the positive momentum direction will be described by the state $\mathrm{E}(k, \uparrow)$ (blue in the lower panel of Fig. 1.3 (c)) whereas the ones moving in the other direction will be described by the state $\mathrm{E}(-k, \downarrow)$ (red in the lower panel of Fig. $1.3(\mathrm{c})$ ). The non-magnetic back-scattering of an electron described by $\mathrm{E}(k, \uparrow)$ into the state $\mathrm{E}(-k, \uparrow)$ is prevented due to the orthogonality of the states $[12,21]$. As briefly introduced above (Fig. 1.1), the advantage of exploiting not only the charge

\footnotetext{
${ }^{3}$ Here, it should be clarified that Majorana zero-energy state in condensed matter physics is not a fermion, but an anyon [22]. The terminology 'Majorana fermions' to refer to Majorana zero-energy modes is misleading. Nevertheless, it is extensively used in literature, thus we use this term here.

${ }^{4}$ The key ingredient for this proposal is based on the braiding (moving particles around in order to perform a quantum computation) of anyons. Not all anyons are directly useful in topological quantum computation; only non-Abelian anyons are useful. Majorana bound states are realization of non-Abelian anyons. A qubit can be realized from two isolated Majorana fermions since each Majorana state is half an electron (an occupied state that would correspond to $|1\rangle$ state) and half a hole (an empty state that would corresponding to $|0\rangle$ state) $[22,23,24]$.
} 


\begin{tabular}{|l|l|}
\hline TI characteristics & Possible technological applications \\
\hline $\begin{array}{l}\text { Helical spin-momentum coupling of the } \\
\text { TI surface states. }\end{array}$ & $\begin{array}{l}\text { Spintronic devices that exploit the spin on the sur- } \\
\text { face. Such devices are believed to reach higher } \\
\text { performance (faster switching) and consume less } \\
\text { energy [6, 20]. }\end{array}$ \\
\hline $\begin{array}{l}\text { Majorana fermion }{ }^{3}: \text { combination of Tls } \\
\text { with ordinary superconductors. }\end{array}$ & $\begin{array}{l}\text { Topological quantum computation, which is a } \\
\text { promising proposal to realize a quantum computer } \\
\text { with robust qubits }{ }^{4}[19] .\end{array}$ \\
\hline
\end{tabular}

Table 1.1: Characteristics of Tls surface/edge states and their potential technological applications.

current, but also the spin current in electronic solid-state devices is something which is attractive in device technology. Table 1.1 gives the proposed applications of topological insulators.

From another perspective, research on TI systems has been motivated by theoretical proposals. The combination of a TI with a superconductor is predicted to lead to the creation of Majorana bound states (MBS) $[28,29]$. The MBS are emergent condensed matter quasi-particles that are analogues to the putative elementary spin-1/2 particles originally proposed by Ettore Majorana in the context of neutrino in particle physics [30]. They possess the intriguing property of being their own anti-particle. The Majorana quasi-particle bound state in condensed matter systems could potentially be used as topological qubit to perform fault-tolerant computation due to the fact that they obey non-abelian statistics [22, 31]. Thus, if Majorana bound states can be engineered, manipulated and their states measured in well-controlled experiments, this could pave the way towards the realisation of a topological quantum computer. A promising route in the search for these exotic fermions is to use 3D Tl-based Josephson junctions $(\mathrm{JJ})$. Figure 1.4 (a) illustrates a JJ composed of two s-wave superconducting electrodes and a TI weak link. In such junctions, the helical nature of the topological surface states is coupled to a conventional s-wave pairing superconductor, which, in turn, induces a spinless p-wave superconducting order parameter component on the $\mathrm{TI}$ surface $[28,32]$. It was shown theoretically that topologically protected, gapless Andreev bound states (ABS), form in this case [19, 28].

Figure 1.4 (b) depicts a typical ABS spectrum for a superconductor-topological insulator Josephson junction. The energy bands of these gapless ABS have $4 \pi$-periodicity in the superconducting phase difference across the junction (blue curve in Fig. 1.4(b)) [33]. This feature is often regarded as the signature of a Majorana bound state (MBS) in a Josephson junction between two s-waves superconductors with a TI barrier. The most experimental progress in looking for MBS signatures 
Page 8 Chapter 1 . Combining the material science and the physics of topological insulators

(a)

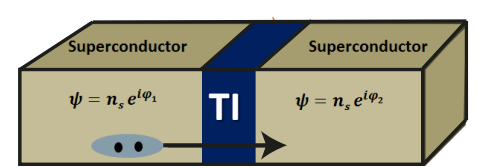

(c)

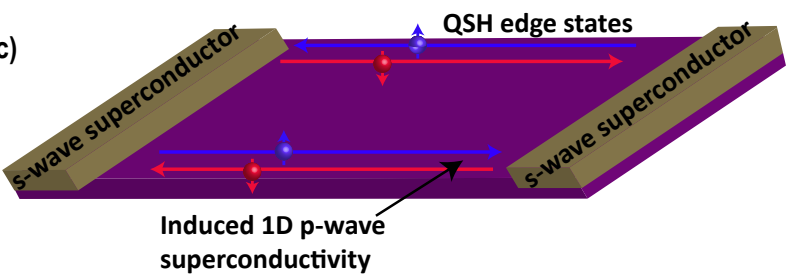

(b)

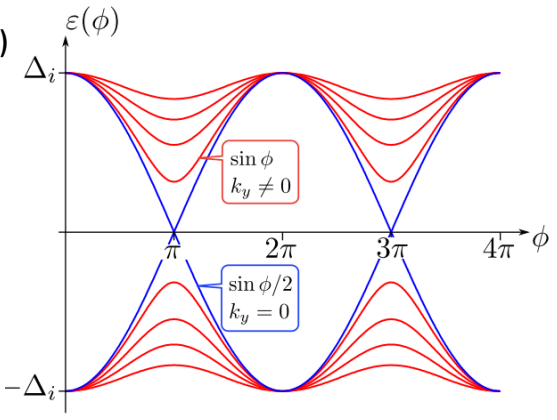

(d)

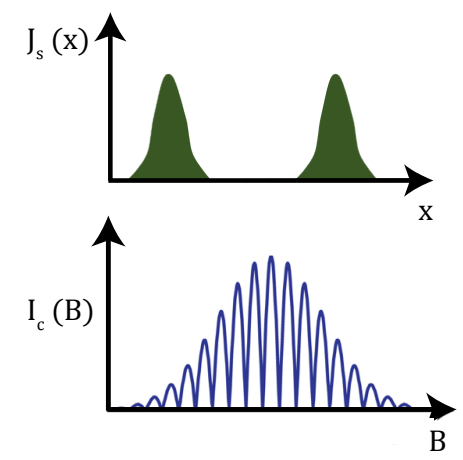

(e)

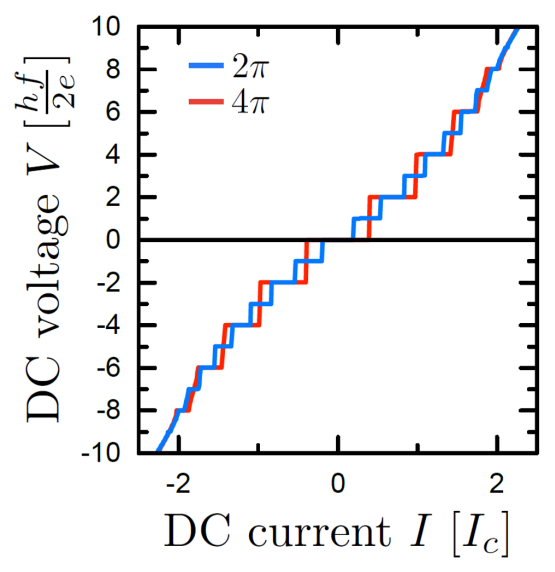

Figure 1.4: Physics of topological insulator Josephson devices (TIJDs). (a) Illustration of a TIJD contacted with two s-wave superconductors. The phase difference between the two superconductors is $\Delta \varphi=\varphi_{2}-\varphi_{1}$. (b) An Andreev bound state spectrum for a 3D TIJD. The blue curve gives the spectrum of the gapless $4 \pi$-periodic topological mode, contributing to the $4 \pi$-supercurrent $I_{4 \pi} \sin \varphi / 2$; whereas the red curve gives the gapped modes contributing to the $2 \pi$-supercurrent $I_{2 \pi} \sin \varphi$ (Fig. from Ref. [25]) (c) Predicted edge suprcurrent in ultra-thin film regime with $p$-wave superconductivity for a topological quantum well system. (d) As the system approach the 2D TI regime with QSHE behaviour of reduced/depleted bulk carriers, the supercurrent density $\left(J_{S}(x)\right)$ develops peaks due to the presence of the helical edge states. When there is a full opening of the gap in the $\mathrm{TI}$ band structure, the supercurrent will be carried only along the helical edge states. The dc-SQUID diffraction pattern is expected. (Fig. from Ref. [26]). (e) I-V characteristics in the presence of microwave ( $\mathrm{rf}$ ) excitation with a $4 \pi$-periodic (red curve) and $2 \pi$-periodic Shapiro steps behaviours (Fig. from Ref. [27]). 
has been on $\ln A$ s or $\operatorname{lnS} b^{5}$ nanowires [34, 35] and $\mathrm{HgTe}$ quantum wells [25]. Even though the preliminary signature of MBS have been recently reported in these systems, another promising alternative route is to use $\mathrm{Bi}$-based $\mathrm{Tls}$ in $2 \mathrm{D}$ regime with quantum spin Hall effect behaviour ${ }^{6}$. To realize this regime, TI thin films would be particularly advantageous: when ultra-thin films in quantum spin Hall insulator regime are used, electrons flow in two counter-propagating 1D edge states of opposite spins (see Fig. 1.4(c)). The $4 \pi$-periodicity features would then appear strongly in TIJDs fabricated on such 2D TI systems, as it has recently been proposed theoretically $[36,37]$ and preliminarily demonstrated experimentally for $\mathrm{JJs}$ fabricated on strained $\mathrm{HgTe}$ films [26, 27]. This is because, the $4 \pi$-periodic ABS in 3D TIJDs is a single-channel effect out of many trivial $2 \pi$-periodic ABS [38]; thus the probability of detecting this topological mode is much more enhanced in 2D TIJDs. Two notable signatures are expected to indicate the presence of MBS on the edges of such 2D TIJDs as illustrated in Fig. 1.4(d) and Fig. 1.4(e). First, in the ac Josephson effect, when phase-locking occurs between the junction dynamics and an external applied microwave radiation, Shapiro steps appear with a voltage equal to $n \hbar \omega / e$, where $n$ is the step index and $\omega$ is the frequency of the applied microwave $[42,43,44]$. In the presence of a $4 \pi$-periodic supercurrent, an unconventional sequence of even steps (see red line in Fig. 1.4(e)), with missing odd steps, is anticipated $[45,46]$. A conventional $2 \pi$-periodic ABS will result in Shapiro steps at $V=n \hbar \omega / 2 e$, which is half the step size of the $4 \pi$-periodic bound state (see blue line in Fig. 1.4(e)). Second, the edge-dominated transport behaviour will be manifested in the interference pattern. When the supercurrent flows only along the helical edge states, the response of the critical current to a magnetic field applied perpendicular to the sample plane is expected to result in a sinusoidal double-slit pattern like a dc-SQUID (superconducting quantum interference device) diffraction pattern [26, 36] (see Fig. 1.4(d)).

Nevertheless, before exploiting these remarkable properties of the TI surface states, one needs first to realize samples with conduction coming from the surface states alone (the chemical potential needs to be placed inside the bulk band gap), which has been proved difficult in $\mathrm{Bi}_{2} \mathrm{Se}_{3}$ and $\mathrm{Bi}_{2} \mathrm{Te}_{3}$, since it requires a careful reduction of the amount of bulk defects in TI materials. These defects are mainly due to vacancies ( $\mathrm{Te}$ or $\mathrm{Se}$ vacancies in the case of $\mathrm{Bi}_{2} \mathrm{Te}_{3}$ and $\mathrm{Bi}_{2} \mathrm{Se}_{3}$, respectively) and anti-site defects in the bulk, thus rendering these materials often electron doped. One approach to overcome this bulk conduction issue in $3 \mathrm{D}$ Tls is to prepare high quality thin films that are

\footnotetext{
${ }^{5}$ These systems go through a topological phase transition under a strong magnetic field [39]. However, there is still an ongoing debate about the topological origin of the observed phenomena since the helical transport in the normal state has not been demonstrated in these systems.

${ }^{6}$ Due to the coupling between the surface states, a finite energy gap is opened in the 2D limit of the 3D TI; and this 2D energy gap is predicted to oscillate between the ordinary insulator gap and the quantum spin Hall gap as a function of thickness $[40,41]$.
} 
Page 10 Chapter 1 . Combining the material science and the physics of topological insulators insulating in the bulk. This dissertation focuses first on the preparation of bulk insulating 3D TI thin films; and then on the investigation of the electronic transport properties of their surface states and the physics of hybrid structures of TI thin films interfaced to superconductors.

\subsection{This Dissertation: Topological insulator thin films and topological Josephson devices}

Bulk conduction in Tls complicate direct exploitation of the surface effects. Furthermore, the existence of strong scatterers (e.g point defects) might create a virtual scattering channel from the surface to the bulk states. To obtain high quality, bulk insulating samples, different research groups have tried several approaches. For single crystals of TI materials, the first strategy was counter-doping of the bulk in $\mathrm{Bi}_{2} \mathrm{Se}_{3}$ and $\mathrm{Bi}_{2} \mathrm{Te}_{3}$ single crystals $[47,48]$ and later the combination of different $\mathrm{TI}$ materials in order to decrease the number of vacancies and defects in prepared single crystals. The materials $\mathrm{Bi}_{2} \mathrm{Te}_{2} \mathrm{Se}[49]$ and $\mathrm{Bi}_{2-x} \mathrm{Sb}_{x} \mathrm{Te}_{3-y} \mathrm{Se}_{y}[50,51]$ are examples where low bulk carrier concentration crystals were obtained using this combination. However, the approach of substitution of $\mathrm{Te}$ and Se with other atoms (like $\mathrm{Sn}$ or $\mathrm{Ca}$ ) and the formation of a nonstoichiometric alloy often resulted in single crystals that have very low surface carrier mobilities, which is a disadvantage for applications. Furthermore, the degradation of the surface transport properties prevents the direct observation of quantum oscillations which are often used to probe details of the TI surface states [52]. Only recently, single crystals of stoichiometric BiSbTeSe $\mathrm{Be}_{2}$ showed distinct surface quantum oscillations [53].

On the other hand, in order to increase the crystal quality and obtain samples with decreased intrinsic defects and high surface mobilities, another approach is to employ evaporation techniques such as molecular beam epitaxy (MBE) which allows the growth of bulk insulating thin films. Using this method, it has been possible to investigate the thickness dependent transport properties $[54,55]$ and band structure behaviour of TI thin films; from which a crossover from $3 \mathrm{D}$ to 2D TI phase have been successively observed in the band structure [40]. Furthermore, MBE grown thin films allowed a direct investigation of the quantum anomalous Hall effect (QAHE) in magnetically doped TIs $[56,57]$. Thus, the high flexibility in selecting and combining different elements, the good control over the film thickness, the possibility of in-situ preparing films and capping them right after growth to avoid extrinsic defects and also the facility of interfacing films with other materials, enabling the creation of several devices and multi-layers on the same film, render MBE grown thin films much more advantageous than bulk single crystals. On this basis, this dissertation focuses on preparing high quality bulk insulating $\mathrm{TI}$ samples in thin film form 
using $\mathrm{MBE}$, and probing their physics through cryogenic electronic transport measurements. We choose to concentrate on the growth of the stoichiometric $\mathrm{Bi}_{2} \mathrm{Te}_{3}$ thin films. This choice was partly motivated by the fact that in contrast to naturally-grown $\mathrm{Bi}_{2} \mathrm{Se}_{3}$ single crystals, which are always electron doped ${ }^{7}$ [58]; $\mathrm{Bi}_{2} \mathrm{Te}_{3}$ crystals can be grown in both $\mathrm{n}$ - and $\mathrm{p}$-types [52, 58]. This is because the $\mathrm{Te}$ anti-site defects $\mathrm{Te}_{\mathrm{Bi}}^{+}$( $\mathrm{Te}$ ion sitting on Bi lattice) or Bi anti-site defects $\mathrm{Bi}_{\mathrm{Te}}^{-}$ (Bi ion sitting the Te lattice) are formed in Te-rich or Bi-rich conditions, respectively [58]. The $\mathrm{Te}_{\mathrm{Bi}}^{+}$is a donor while $\mathrm{Bi}_{\mathrm{Te}}^{-}$is an acceptor, suggesting that through a careful control of the growth conditions, it would be possible to prepare bulk insulating $\mathrm{Bi}_{2} \mathrm{Te}_{3}$ thin films.

$\mathrm{Bi}_{2} \mathrm{Te}_{3}$ has a rhombohedral crystal structure, which consists of quintuple layers bound to each other (atomic layers of Te[1]-Bi-Te[2]-Bi-Te[1]) through weak Van der Waals forces, giving easy access to the (111) surface by cleavage. Fig. 1.5(a) depicts the crystal structure of $\mathrm{Bi}_{2} \mathrm{Te}_{3}$. Strong evidence of topological surface states in $\mathrm{Bi}_{2} \mathrm{Te}_{3}$ has been obtained mainly using surfacesensitive techniques like angle-resolved photoemission spectroscopy (see Fig. 1.5(b)) and scanning tunneling microscopy (see Fig. 1.5(c)). While many topological insulator materials have been theoretically predicted and experimentally proven so far [58], the stoichiometric bismuth chalcogenides, especially $\mathrm{Bi}_{2} \mathrm{Te}_{3}$, is one of the most extensively studied, not only for its thermoelectric potential applications [59,60], but also for its $\mathrm{TI}$ behaviour $[61,62,63]$. However, even though this system is largely investigated (both in single crystals and thin film form), only few studies have unambiguously managed to show intrinsic conduction behaviour through topological surface states of insulating $\mathrm{Bi}_{2} \mathrm{Te}_{3}$ samples $[16,63,64,65]$. It is only recently that good bulk insulator $\mathrm{Bi}_{2} \mathrm{Te}_{3}$ thin films with metallic surface states have been prepared and their transport and electronic band structure were investigated using in-situ four-point probe conductivity and angle resolved photoemission spectroscopy, respectively [65]. Compared to its prototypical material $\mathrm{Bi}_{2} \mathrm{Se}_{3}$, even though both materials share the same rhombohedral crystal structure and show a warping of the surface state Dirac cone along the $\Gamma-\mathrm{M}$ direction away from the Dirac point, the warping in $\mathrm{Bi}_{2} \mathrm{Te}_{3}$ is much more pronounced [66], rendering the band structure of $\mathrm{Bi}_{2} \mathrm{Te}_{3}$ to be richer. Also, the Dirac point of $\mathrm{Bi}_{2} \mathrm{Te}_{3}$ lies in a local minimum of the bulk valence band [61, 63].

In the first place, this dissertation aims to identify the conditions under which molecular beam epitaxy gives stable and reproducible results for obtaining stoichiometric and bulk-insulating $\mathrm{Bi}_{2} \mathrm{Te}_{3}$ thin films. Chapter 2 presents details on setting up the MBE growth system, optimization of the growth parameters and the growth mode of $\mathrm{Bi}_{2} \mathrm{Te}_{3}$ thin films on different substrates. Here, the essential point is to prepare films in a Te rich environment, to achieve a full distillation ${ }^{8}$

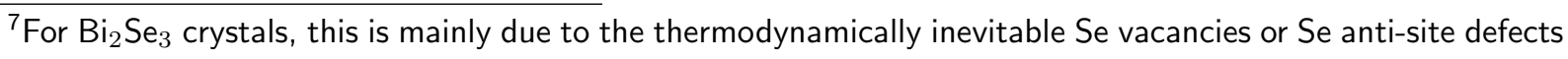
that have low formation energies $[67,68]$.

${ }^{8}$ We used the MBE distillation conditions [70]. With this procedure, the excess Te is re-evaporated from the
} 
(a)

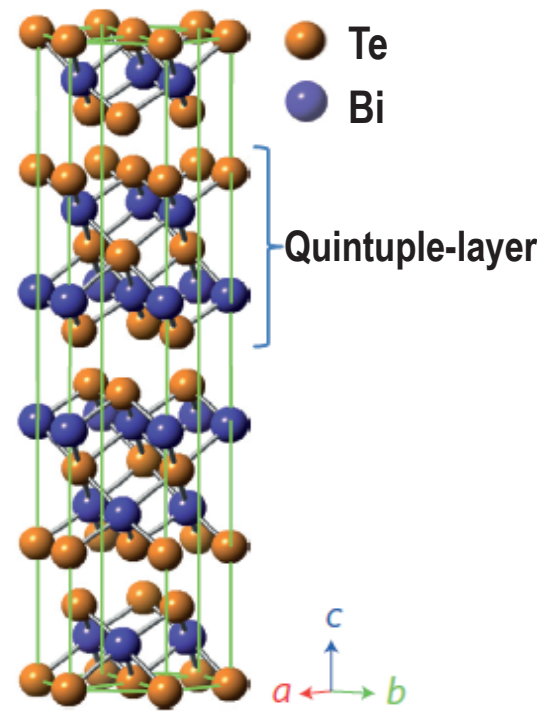

(b)
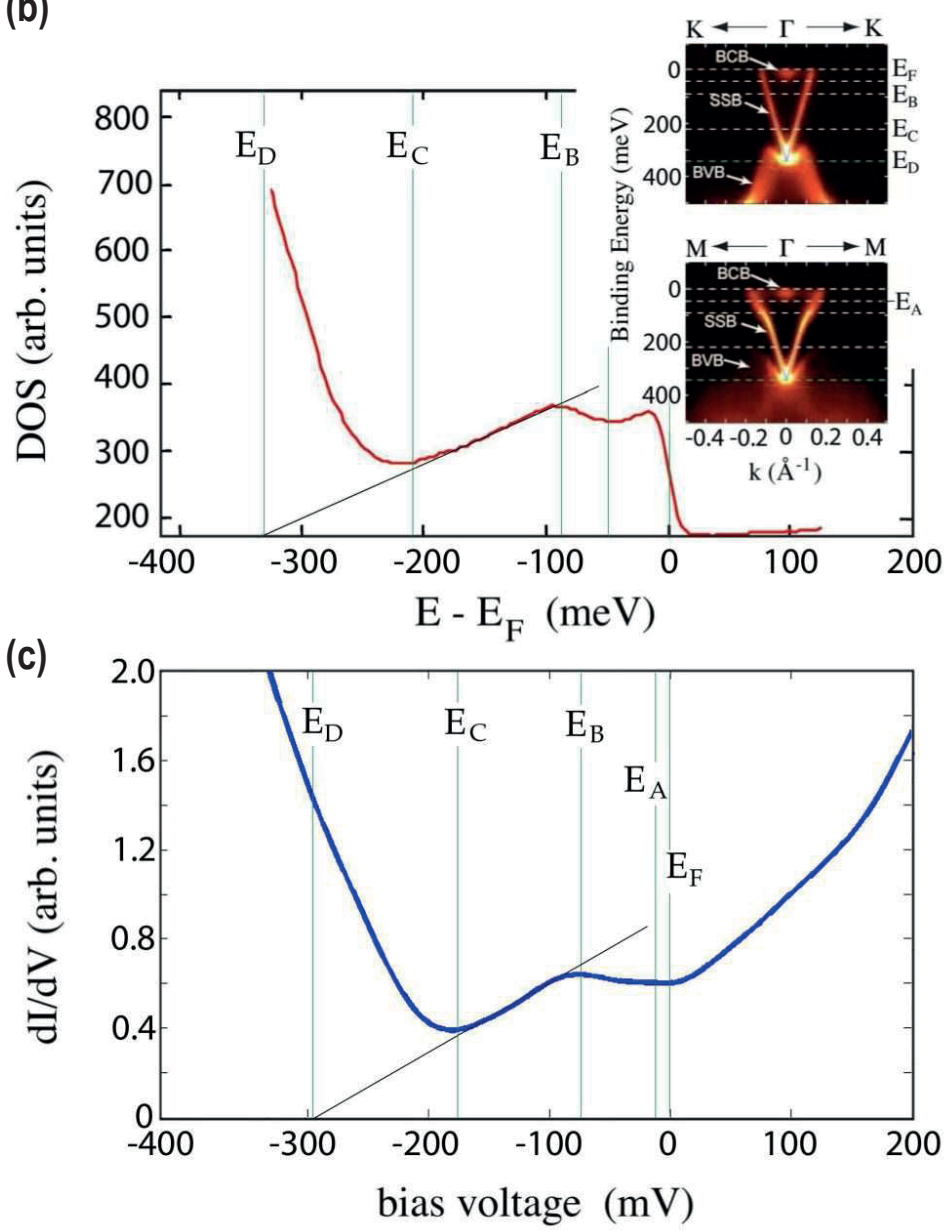

Figure 1.5: Evidence of topological surface states in $\mathrm{Bi}_{2} \mathrm{Te}_{3}$ samples. (a) The rhombohedral crystal structure of $\mathrm{Bi}_{2} \mathrm{Te}_{3}$, which consists of a stack of quintuple layers (QL) bonded together by Van der Waals forces. The height of each QL is $1 \mathrm{~nm}$ (Fig. from Ref. [69]). (b) Integrated density of states from ARPES. The linear behaviour in DOS is related to the topological linear Dirac surface states. (right inset) ARPES spectra along the $\Gamma-M$ and the $\Gamma-K$ directions. Along the $\Gamma-M$ direction, there is a clear outward bending of the Dirac surface states due to the hexagonal warping effect in $\mathrm{Bi}_{2} \mathrm{Te}_{3}$. (c) Typical scanning tunnelling spectroscopy STS spectrum of $\mathrm{B}_{2} \mathrm{Te}_{3}$ sample, also showing clear linear Dirac surface states (Fig. from Ref. [62]). These data are from a $0.27 \% \mathrm{Sn}$-doped single crystal of $\mathrm{Bi}_{2} \mathrm{Te}_{3}$. It is clear that even after the counter doping procedure, the Fermi level lies at the bottom of the conduction band.

process for the $\mathrm{Te}$, and also to employ a two-temperature growth procedure in order to avoid disordered interfacial layers at the interfaces between the films and the substrate so that high film by choosing an appropriate elevated substrate temperature. 
quality, single domain crystalline films can be obtained.

To investigate the physics of the obtained thin films, in Chapter 3 we present a magnetotransport study of $\mathrm{Bi}_{2} \mathrm{Te}_{3}$ thin films exposed to ambient conditions. The trends observed in magnetotransport reflect the coexistence of bulk and surface channels in measured samples. A fit to the two band model for the measured Hall signal $R_{x y}$ did not correctly reproduce the longitudinal resistance behaviour. Using the helical magnetoresistance model, we are able to fit the longitudinal and transverse conductivity data simultaneously. Analysis of observed quantum oscillations suggest a significant contribution of bulk states to the measured quantum oscillations.

In the second place, this dissertation focuses on performing a systematic in situ-characterization of intrinsic $\mathrm{Bi}_{2} \mathrm{Te}_{3}$ thin films in order to investigate the effect of aging/degradation due to any vacancies or antisite defects for films stored in vacuo. Furthermore, using in-situ surface sensitive techniques, the effect of pure oxygen exposure at atmospheric pressure; and then the effect of ex-situ contamination in air at the surface of intrinsic $\mathrm{Bi}_{2} \mathrm{Te}_{3}$ was investigated. This study is relevant since samples are exposed to ambient conditions for most magnetotransport studies and fabrication processes. Chapter 4 presents details on in-situ spectroscopy of our thin films and the impact of extrinsic defects. Here, we demonstrate the absence of a conducting bulk shunt in our $\mathrm{Bi}_{2} \mathrm{Te}_{3}$ thin films grown on different substrates $\left(\mathrm{SrTiO}_{3}\right.$ [111] and $\left.\mathrm{Al}_{2} \mathrm{O}_{3}[0001]\right)$ by a combination of in-situ scanning tunnelling microscopy and in-situ angle resolved photoemission spectroscopy. We find that the surface morphology and electronic band structure of our films are not affected by in-vacuo storage and exposure to oxygen, whereas major changes are observed when the sample is exposed to ambient conditions. Thus, there is a need to protect the surface states from degradation and unintentional doping due to extrinsic defects.

Backed by the conclusions of Chapter 4 , in Chapter 5 we determine the proper in-situ capping procedure of intrinsic $\mathrm{Bi}_{2} \mathrm{Te}_{3}$ thin films grown on different insulating substrates. Using two different capping materials $\left(\mathrm{Al}_{2} \mathrm{O}_{3}\right.$ or Te layer), we present magnetotransport characterizations together with a gate-dependent electronic transport study for in-situ capped films. We find that both capping materials help protecting $\mathrm{TI}$ surface states and achieving full depletion of bulk carriers, thus allowing access to the topological transport regime dominated by surface state conduction. In addition, when the Fermi level is placed in the bulk band gap, we observe the presence of two coherent conduction channels associated with the two decoupled surfaces.

In the third place, motivated by the fact that we can prepare high quality $\mathrm{Bi}_{2} \mathrm{Te}_{3}$ thin films on different insulating substrates with a remarkable low level of intrinsic doping in the bulk (Chapter 4 and Chapter 5); and inspired by recent theoretical proposals about the detection of unconventional Josephson effects related to Majorana physics in gapped TIJDs [36, 37]; we set 
Page 14 Chapter 1. Combining the material science and the physics of topological insulators out to investigate the Josephson physics in hybrid superconductor-TI structures. We used thinner $(\leq 15 \mathrm{~nm})$ thin films of $\mathrm{Bi}_{2} \mathrm{Te}_{3}$ as weak link since a crossover from 3D TI to a $2 \mathrm{D} \mathrm{TI}$ with helical edge states between two superconductors is expected in this regime. In Chapter 6, we present an in-depth analysis and discussion on combined gate-tunable TIJDs and normal state transport in $\mathrm{Bi}_{2} \mathrm{Te}_{3} \mathrm{TI}$ films. Since the samples were grown on insulating substrates with a high relative dielectric constant $\left(\mathrm{SrTiO}_{3}[111]\right)$, we investigate the modulation of both the supercurrent and the sample's carrier density as the films are bottom-gated using STO substrate as a back gate. One of the advantages of using thin films over single crystals is their design flexibility allowing the fabrication of several JJs alongside Hall bar devices on the same sample. The final Chapter 7 is an epilogue, in which the results are discussed in a wider perspective and an outlook on future experiments is presented. 


\section{Bibliography}

[1] G. E. Moore, Proc. of the IEEE 86, (1998).

[2] M. N. Baibich, J. M. Broto, A. Fert, F. Nguyen Van Dau, F. Petroff, P. Etienne, G. Creuzet, A. Friederich, and J. Chazelas, Phys. Rev. Lett. 61, 2472 (1988).

[3] G. Binasch, P. Grünberg, F. Saurenbach, and W. Zinn. Phys. Rev. B 39, 4828 (1989).

[4] J .S. Moodera, L. R. Kinder, T.M. Wong, R. Meservey. Phys. Rev. Lett. 74, 3273 (1995).

[5] D. D. Awschalom and M. E. Flatté, Nat. Phys. 3, 153 (2007).

[6] I. Žutić, J. Fabian, and S. D. Sarma, Rev. Mod. Phys. 76, 323 (2004).

[7] G. Schmidt and L. W. Molenkamp, Semicond. Sci. Technol. 17, 310 (2002).

[8] G. Schmidt, J. Phys. D: Appl. Phys. 38, R107 (2005).

[9] O. V. Yazyev, J. E. Moore and S. G. Louie, Phys. Rev. Lett. 105, 266806 (2010).

[10] M. Zeng and G. Liang, J. Appl. Phys. 112, 073707 (2012).

[11] D. Hsieh, Y. Xia, D. Qian, L. Wray, J. H. Dil, F. Meier, J. Osterwalder, L. Patthey, J. G. Checkelsky, N. P. Ong, A. V. Fedorov, H. Lin, A. Bansil, D. Grauer, Y. S. Hor, R. J. Cava and M. Z. Hasan, Nature 460, 1101 (2009).

[12] C. Jozwiak, C. -H. Park, K. Gotlieb, Ch. Hwang, D. -H. Lee, S. G. Louie, J. D. Denlinger, C. R. Rotundu, R. J. Birgeneau, Z. Hussain and A. Lanzara, Nature Phys. 9, 293 (2013).

[13] C. L. Kane and E. J. Mele, Phys. Rev. Lett. 95, 226801 (2005).

[14] L. Fu, C. L. Kane, and E. J. Mele, Phys. Rev. Lett. 98, 106803 (2007).

[15] M. Nakahara, Geometry, Topology and Physics, $2^{\text {nd }}$ edition, Institute of Physics Publishing, Bristol (UK) and Philadelphia (USA) 2003. 
[16] P. Ngabonziza, R. Heimbuch, N. de Jong, R. A. Klaassen, M. P. Stehno, M. Snelder, A. Solmaz, S. V. Ramankutty, E. Frantzeskakis, E. van Heumen, G. Koster, M. S. Golden, H. J. W. Zandvliet, and A. Brinkman, Phys. Rev. B 92, 035405 (2015).

[17] J. E. Moore and L. Balents, Phys. Rev. B 75, 121306 (2007).

[18] L. Fu and C. L. Kane, Phys. Rev. B 76, 045302 (2007).

[19] M. Z. Hasan and C. L. Kane, Rev. Mod. Phys. 82, 3045 (2010).

[20] S. Das Sarma, Spintronics. American Scientist 89, 516 (2000).

[21] Z.-H. Pan, E. Vescovo, A. V. Fedorov, D. Gardner, Y. S. Lee, S. Chu, G. D. Gu, and T. Valla, Phys. Rev. Lett. 106, 257004 (2011).

[22] C. Nayak, S. H. Simon, A. Stern, M. Freedman, and S. Das Sarma, Rev. Mod. Phys. 80, 1083 (2008).

[23] S. Das Sarma, M. Freedman and C. Nayak, Nat. Quant. Inf. 1, 15001 (2015).

[24] S. -H. Ho, S. -P. Chao, C. -H. Chou and F. -L. Lin, New J. Phys. 16, 113062 (2014).

[25] J. Wiedenmann, E. Bocquillon, R. S. Deacon, S. Hartinger, O. Herrmann, T. M. Klapwijk, L. Maier, C. Ames, C. Brüne, C. Gould, A. Oiwa, K. Ishibashi, S. Tarucha, H. Buhmann and L. W. Molenkamp, Nat. Commun. 7, 10303 (2016).

[26] S. Hart, H. Ren, T. Wagner, P. Leubner, M. Mühlbauer, C. Brüne, H. Buhmann, L. W. Molenkamp and A. Yacoby, Nat. Phys. 10, 638 (2014).

[27] E. Bocquillon, R. S. Deacon, J. Wiedenmann, P. Leubner, T. M. Klapwijk, C. Brüne, K. Ishibashi, H. Buhmann, and L. W. Molenkamp, http://arxiv.org/abs/1601.08055 (2016).

[28] L. Fu and C. L. Kane. Phys. Rev. Lett. 100, 096407 (2008).

[29] A. R. Akhmerov, Johan Nilsson, and C. W. J. Beenakker, Phys. Rev. Lett. 102, 216404 (2009).

[30] E. Majorana, Nuovo Cim. 14, 171 (1937).

[31] A. Y. Kitaev, Ann. Phys. 303, 2 (2003).

[32] A. C. Potter and P. A. Lee Phys. Rev. B 83, 184520 (2011). 
[33] Y. Tanaka, T. Yokoyama, and N. Nagaosa, Phys. Rev. Lett. 103, 107002 (2009).

[34] V. Mourik, K. Zuo, S. M. Frolov, S. R. Plissard, E. P. A. M. Bakkers, and L. P. Kouwenhoven, Science, 336, 1003 (2012).

[35] L. P. Rokhinson, X. Liu, and J. K. Furdyna, Nat. Phys. 8, 795 (2012).

[36] G. Tkachov, P. Burset, B. Trauzettel, and E. M. Hankiewicz, Phys. Rev. B 92, 045408 (2015).

[37] J. Song, H. Liu, J. Liu, Y. Li, R. Joynt, Q. -F. Sun, and X. C. Xie, Phys. Rev. B 93, 195302 (2016).

[38] M. Snelder, M. Veldhorst, A. A. Golubov, A. Brinkman Physical Review B 87, 104507 (2013).

[39] C. W. J. Beenakker, An. Rev. of Cond. Mat. Phys., 4, 113 (2013) and references therein.

[40] Y. Zhang, K. He, C. Z. Chang, C. L. Song, L. L. Wang, X. Chen, J. F. Jia, Z. Fan, X. Dai, W. Y. Shan, S. Q. Shen, Q. Niu, X. L. Qi, S. C. Zhang, X. C. Ma and Q. K. Xue, Nat. Phys. 6, 584 (2010).

[41] C. -X. Liu, H. Zhang, B. Yan, X. -L. Qi, T. Frauenheim, X. Dai, Z. Fang, and S. -C. Zhang, Phys. Rev. B 81, 041307(R) (2010).

[42] L. Fu and C. L. Kane, Phys. Rev. Lett. 102, 216403 (2009).

[43] D. M. Badiane, M. Houzet and J. S. Meyer, Phys. Rev. Lett. 107, 177002 (2011).

[44] S. Shapiro, Phys. Rev. Lett. 11, 80 (1963).

[45] P. San-Jose, E. Prada and R. Aguado, Phys. Rev. Lett. 108, 257001 (2012).

[46] M. Houzet, J. S. Meyer, D. M. Badiane and L.I. Glazman, Phys. Rev. Lett. 111, 046401 (2013).

[47] J. G. Checkelsky, Y. S. Hor, M. -H. Liu, D. -X. Qu, R. J. Cava, and N. P. Ong, Phys. Rev. Lett. 103, 246601 (2009).

[48] S. S. Hong, J. J. Cha, D. Kong and Y. Cui, Nat. Commun. 3, 757 (2012).

[49] Z. Ren, A. A. Taskin, S. Sasaki, K. Segawa, and Y. Ando, Phys. Rev. B 82, 241306(R) (2010). 
[50] Z. Ren, A. A. Taskin, S. Sasaki, K. Segawa, and Y. Ando, Phys. Rev. B 84, 165311 (2011).

[51] T. Arakane, T. Sato, S. Souma, K. Kosaka, K. Nakayama, M. Komatsu, T. Takahashi, Z. Ren, K. Segawa and Y. Ando, Nat. Commun. 3, 636 (2012).

[52] D. -X. Qu, Y. S. Hor, J. Xiong, R. J. Cava, N. P. Ong, Science 329, 821 (2010).

[53] Y. Xu, I. Miotkowski, C. Liu, J. Tian, H. Nam, N. Alidoust, J. Hu, C.-K. Shih, M. Z. Hasan and Y. P. Chen, Nat. Phys. 10, 956 (2014).

[54] Y. S. Kim, M. Brahlek, N. Bansal, E. Edrey, G. A. Kapilevich, K. lida, M. Tanimura, Y. Horibe, S.-W. Cheong, and S. Oh, Phys. Rev. B 84, 073109 (2011).

[55] N. Bansal, Y. S. Kim, M. Brahlek, E. Edrey, and S. Oh, Phys. Rev. Let. 109, 116804 (2012).

[56] C. -Z. Chang, J. Zhang, X. Feng, J. Shen, Z. Zhang, M. Guo, K Li, Y. Ou, P. Wei, L. -L. Wang, Z. -Q. Ji, Y. Feng, S. Ji, X. Chen, J. Jia, X. Dai, Z. Fang, S. -C. Zhang, K. He, Y. Wang, L. Lu, X. -C. Ma, Q. -K. Xue, Science 340, 167 (2013).

[57] C. -Z. Chang, W. Zhao, D. Y. Kim, H. Zhang, B. A. Assaf, D. Heiman, S. -C. Zhang, C. Liu, M. H. W. Chan and J. S. Moodera, Nat. Mater. 14, 473 (2015).

[58] Y. Ando, J. Phys. Soc. Jpn. 82, 102001 (2013), and references therein.

[59] M. Scheele, N. Oeschler, K. Meiern, A. Kornowski, C. Klinke, H. Weller, Adv. Funct. Mater. 19, 3476 (2009).

[60] W. Xie, X. Tang, Y. Yan, Q. Zhang, T. M. Tritt, J. Appl. Phys. 105, 113713 (2009).

[61] Y. L. Chen, J. G. Analytis, J. -H. Chu, Z. K. Liu, S. -K. Mo,X. L. Qi, H. J. Zhang, D. H. Lu, X. Dai, Z. Fang, S. C. Zhang, I. R. Fisher, Z. Hussain, Z. -X. Shen, Science 325, 178 (2009).

[62] Z. Alpichshev, J. G. Analytis, J. -H. Chu, I. R. Fisher, Y. L. Chen, Z. X. Shen, A. Fang, and A. Kapitulnik, Phys. Rev. Lett. 104, 016401 (2010).

[63] Y. Li, G. Wang, X. Zhu, M. Liu, C. Ye, X. Chen, Y. Wang, K. He, L. Wang, X. Ma, H. Zhang, X. Dai, Z. Fang, X. Xie, Y. Liu, X. Qi, J. Jia, S. Zhang, and Q. Xue, Adv. Funct. Mater. 22, 4002 (2010).

[64] G. Wang, X. Zhu, Y. Sun, Y. Li, T. Zhang, J. Wen, X. Chen, K. He, L. Wang, X. Ma, J. Jia, S. B. Zhang, and Q. Xue, Adv. Funct. Mater. 23, 2929 (2011). 
[65] K. Hoefer, C. Becker, D. Rata, J. Swanson, P. Thalmeier, and L. H. Tjeng, Proc. Natl. Acad. Sci. USA 111, 14979 (2014).

[66] M. Nomura, S. Souma, A. Takayama, T. Sato, T. Takahashi, K. Eto, K. Segawa, and Y. Ando, Phys. Rev. B 89, 045134 (2014).

[67] D. O. Scanlon, P. D. C. King, R. P. Singh, A. de la Torre, S. M. Walker, G. Balakrishnan, F. Baumberger, and C. R. A. Catlow, Adv. Mater. 24, 2154 (2012).

[68] L. -L. Wang, M. Huang, S. Thimmaiah, A. Alam, S. L. Budko, A. Kaminski, T. A. Lograsso, P. Canfield, and D. D. Johnson: Phys. Rev. B 87, 125303 (2013).

[69] D. Kong, Y. Chen, J. J. Cha, Q. Zhang1, J. G. Analytis, K. Lai, Z. Liu, S. S. Hong, K. J. Koski, S.-K. Mo, Z. Hussain, I. R. Fisher, Z. -X Shen, and Y. Cui, Nat. Nanot. 6, 705 (2011).

[70] R. Sutarto, S. G. Altendorf, B. Coloru, M. Moretti Sala, T. Haupricht, C. F. Chang, Z. Hu, C. Schüßler-Langeheine, N. Hollmann, H. Kierspel, H. H. Hsieh, H. -J. Lin, C. T. Chen, and L. H. Tjeng, Phys. Rev. B 79, 205318 (2009). 



\section{2}

\section{Growth and surface morphology of $\mathrm{Bi}_{2} \mathrm{Te}_{3}$ topological insulator thin films}

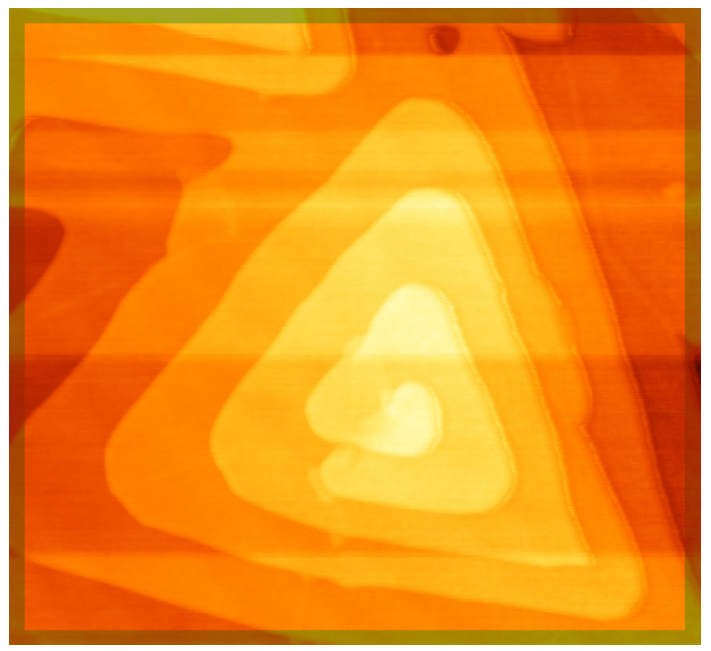

This chapter introduces the molecular beam epitaxy growth and morphology of bismuth telluride topological insulator thin films using molecular beam epitaxy. We discuss the choice of substrates and surface treatment scheme. A detailed description of the molecular beam epitaxy system and of the optimization process towards growth of high quality films are given. In-situ experimental tools employed for thin films characterization are introduced. Additionally, the morphology of $\mathrm{Bi}_{2} \mathrm{Te}_{3}$ thin films is assessed using atomic force microscopy.

Part of the work discussed in this chapter will be published as a book chapter in the Springer book series on "In-situ Characterization Techniques for Nanomaterials" as:

P. Ngabonziza, M. P. Stehno, G. Koster, and A. Brinkman "In-situ Characterization Tools for Topological Insulator Nanomaterials" 
Page 22 Chapter 2. Growth and surface morphology of $\mathrm{Bi}_{2} \mathrm{Te}_{3}$ topological insulator thin films

\subsection{Substrate selection and treatment}

The lattice constant mismatch between the substrate and the film is a major challenge in realizing various kinds of epitaxial heterostructures [1]. A large lattice mismatch between the constituent materials leads to defects and worsens the crystal quality of the epitaxially grown film. However, for $\mathrm{Bi}_{2} \mathrm{Te}_{3}$ and other 3D TI systems, the lattice-match criteria are drastically relaxed since the unit cells are coupled by Van der Waals interactions. This growth preference is known as Van der Waals epitaxy $[1,2,3]$. Thus, a variety of substrates can be chosen for the growth of $\mathrm{TI}$ thin films, and relatively good films have been obtained. Substrates reported so far include CdS [0001] [4, 5], Si [111] $[6,7,8] \mathrm{Al}_{2} \mathrm{O}_{3}[0001][9,10,11], \mathrm{SrTiO}_{3}[11,12,13,14]$, GaAs [111]B [68], GaN [16], InP [17, 18, 19], graphene [20], $\mathrm{CaF}_{2}$ [21] and many others [22, 23].

To avoid the contribution of conduction from the substrate, the substrate should be insulating. Thus, for the growth of $\mathrm{Bi}_{2} \mathrm{Te}_{3}$ thin films, we chose to use substrates that remain insulating after all the processing steps so that we avoid possible parallel conductance paths. In addition, insulating substrates with a high relative dielectric constant at low temperatures, offer the opportunity of gate-tuning the film transport properties using the substrates as a back gate [24]. Films presented in this dissertation were grown on insulating substrates, either $\mathrm{Al}_{2} \mathrm{O}_{3}$ [0001] (sapphire) or $\mathrm{SrTiO}_{3}$ [111] (STO). The lattice mismatch between $\mathrm{Bi}_{2} \mathrm{Te}_{3}$ and sapphire is $8.7 \%$ and $-10.8 \%$ for $\mathrm{SrTiO}_{3}[111][22,23]$.

Even though the atomically well-defined single-termination of the substrates surface is not a requirement, proper surface preparation is desirable to reach atomically flat surfaces. This helps in obtaining sharp interfaces between substrate and film, reducing the film roughness and thus resulting in better transport characteristics. The protocol used to obtain atomically well-defined $\mathrm{Al}_{2} \mathrm{O}_{3}$ [0001] surface starts with ultrasonic immersion of the as-received substrate in acetone for 5$10 \mathrm{~min}$ to remove particulates or residues at the surface from the dicing process. The substrates were then rinsed with ethanol and dried using a nitrogen gas gun. Thereafter, the substrates were annealed at a temperature of $1050{ }^{\circ} \mathrm{C}$ for 1 hour. Longer annealing time (2.5 and 5 hours) produced insignificant changes to the surface topography of the $\mathrm{Al}_{2} \mathrm{O}_{3}$ substrates. Figure 2.1(a) shows the surface of the thermally annealed $\mathrm{Al}_{2} \mathrm{O}_{3}$ [0001] substrate. We observe a smooth surface with step-terrace structures of a width of $\sim 100 \mathrm{~nm}$. On the other hand, to obtain smooth STO [111] substrates, we combined chemical etching and thermal annealing as described in Ref. [25]. The configuration of the STO [111] surface is constituted of alternately stacked charged $\mathrm{SrO}_{3}^{4-}$ and $\mathrm{Ti}^{4-}$ atomic layers (see Fig. 2.1(b)). It is possible to obtain single termination from this configuration by using a separation of Sr- and Ti-related layers, followed by a selective etching 


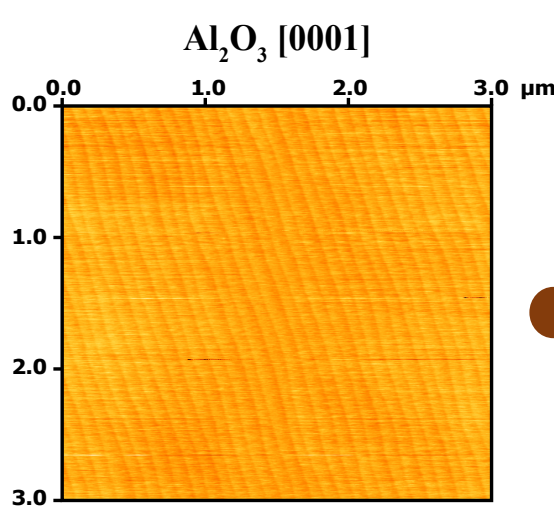

(a)

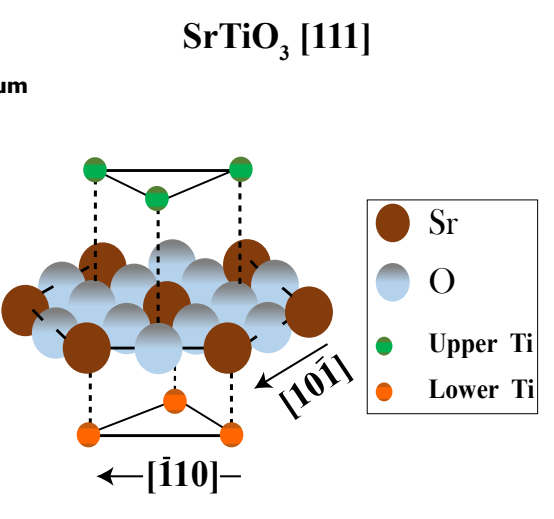

(b)

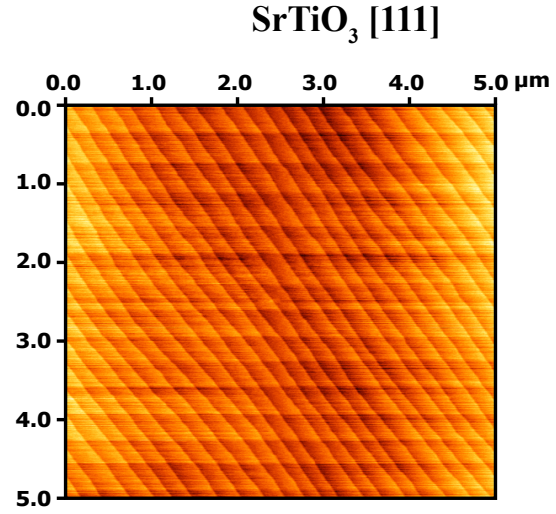

(c)

Figure 2.1: Substrate treatment (a) $3 \times 3 \mu \mathrm{m}$ atomic force microscopy (AFM) image of $\mathrm{Al}_{2} \mathrm{O}_{3}$ [0001] substrate after annealing for 1 hour. (b) An illustration of the atomic configuration of an STO [111] surface. The upper and the lower $\mathrm{Ti}$ indicate $\mathrm{Ti}^{4+}$ ions located above and below the $\mathrm{SrO}_{3}^{4-}$ plane, respectively. The distance between the upper and lower $\mathrm{Ti}^{4+}$ planes is $0.23 \mathrm{~nm}$ [25]. (c) $5 \times 5 \mu \mathrm{m} \mathrm{AFM} \mathrm{image} \mathrm{of} \mathrm{SrTiO}_{3}$ [111] after combined chemical etching and thermal annealing procedure. All substrates show atomically smooth terraces of predominantly unit-cell step heights.

step. This selectiveness is because the Sr-related compounds formed in water are known to be easily soluble in water and acids, whereas the Ti-related compounds are not expected to react with water or most acids (except with $\mathrm{H}_{2} \mathrm{SO}_{4}$ ) [26]. First, the as-received substrates are cleaned in an ultrasonic bath in ethanol for 5 minutes to remove particulates. Subsequently, substrates are dipped in ultrasonically agitated hot $\left(80^{\circ} \mathrm{C}\right)$ de-ionized water for 20-30 min to realize the selective etching of the $\mathrm{SrO}_{3}^{4-}$ layer. Next, the substrates are placed in an ultrasonically agitated buffered hydrogen fluoride (BHF) solution for 30 seconds to completely remove Sr-related compounds. Lastly, substrates are annealed in a tube furnace at $950{ }^{\circ} \mathrm{C}$ in $\mathrm{O}_{2}$ flow for 1 to 2 hours in order to facilitate the diffusion of the surface atoms. Figure 2.1(c) depicts an atomic force microscope (AFM) image of the $\mathrm{SrTiO}_{3}$ [111] substrate after this combined chemical and thermal annealing treatment. The resulting substrates show a clear step-and-terrace structure, with uniform step height of about $2.7 \mathrm{~nm}$, signifying a single-terminated STO [111] surface. For all data presented in this dissertation, the sapphire and STO [111] substrates were cleaned using the procedure presented above. The existence of clean surfaces with single atomic termination, investigated using ex-situ AFM, was a condition for selecting substrates employed later for thin film growth. 
Page 24 Chapter 2. Growth and surface morphology of $\mathrm{Bi}_{2} \mathrm{Te}_{3}$ topological insulator thin films

\subsection{Thin films deposition and characterization}

\subsubsection{COMAT system}

To grow our thin films, we used the molecular beam epitaxy (MBE) technique. The MBE system, we used to prepare samples is part of a multi-purpose cluster tool, called COMAT (Complex Oxide MATerials) system at the University of Twente (see Fig. 2.2). This multi-techniques system is composed of different ultra-high vacuum chambers for thin film growth: MBE and pulsed laser deposition (PLD); and thin film characterizations: X-ray photoelectron spectroscopy (XPS) and low-temperature scanning four-probe microscope (SPM). The individual UHV growth and analysis chambers are connected via a distribution chamber (DC), which facilitates an in-situ growth and subsequent characterization without breaking the vacuum. The distribution chamber is also used to store various thin films in vacuo before being transferred to another in-situ setup for subsequent analysis or taken ex-situ for additional films characterizations and measurements. Here, we focus on describing the e-beam evaporator chamber used for growing $\mathrm{TI}$ thin films. For more details on the other experimental techniques (PLD, XPS and SPM), a short introduction is presented below (section 2.2), and the interested reader can consult available references $[27,28,29,30]$ on these techniques.

To load samples (mainly fresh substrates on which to grow films) into the system, they are first introduced into the load lock chamber (LLC) and the load lock is pumped down to a pressure of $10^{-7}$ to $10^{-8}$ mbar. The sample can then be transferred into the DC. Once in the DC, using a wobble stick (labelled (a) in Fig. 2.2), they can be placed on a sample heater and then moved to the evaporation system using a magnetic transfer stick (labelled (b) in Fig. 2.2). The MBE chamber is equipped with a turbo molecular pump backed by a nonevaporable getter (NEG) pump [31], (labelled (c) in Fig. 2.2) and it is kept at a base pressure lower than $5 \times 10^{-10}$ mbar. In order to have relatively accurate and homogeneous temperature control on the substrate, an UHV screw-driver (labelled (d) in Fig. 2.2) is used to properly tighten the substrate holder onto the heater surface. The heater together with the substrate clamped on it are then place onto the heater stage.

\subsubsection{Electron beam evaporation}

Thin films of $\mathrm{Bi}_{2} \mathrm{Te}_{3}$ have been synthesized recently, as three-dimensional Tls, using different experimental methods. The most widely used techniques are MBE [22, 32, 33], PLD [34, 35, 36], 


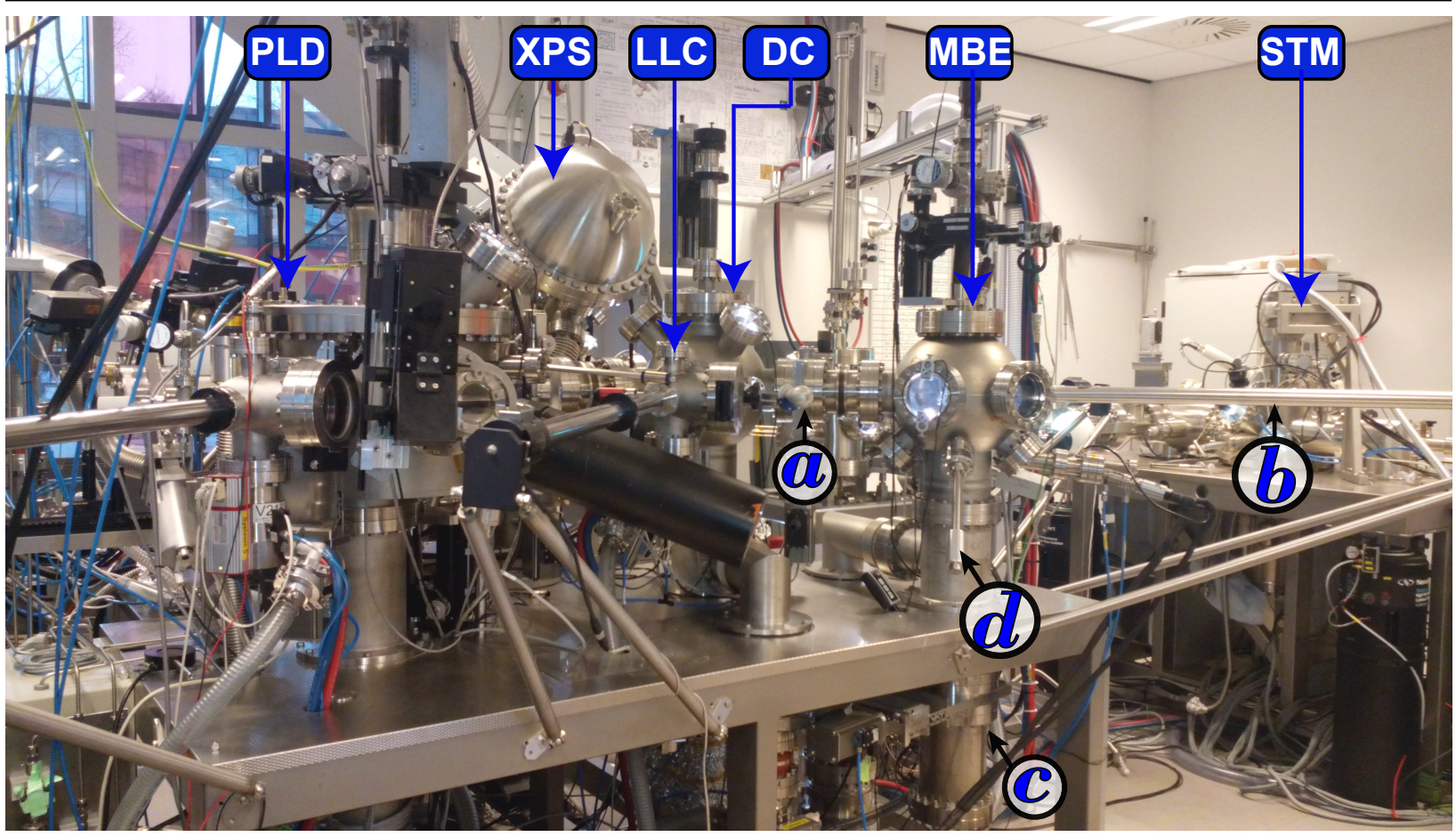

Figure 2.2: The COMAT system. Photograph of the combined vacuum system where different vacuum chambers are indicated. The base pressure of each system is: $\mathrm{MBE}\left(<5 \times 10^{-10} \mathrm{mbar}\right)$, PLD $\left(5 \times 10^{-8} \mathrm{mbar}\right)$, XPS $\left(<5 \times 10^{-11} \mathrm{mbar}\right), \operatorname{STM}\left(<1.2 \times 10^{-11} \mathrm{mbar}\right)$ and the DC $\left(5 \times 10^{-10} \mathrm{mbar}\right)$.

metal-organic chemical vapour deposition (MOCVD) [37] and hot wall epitaxy (HWE) [38]. Amongst these techniques, MBE has been especially established to grow high-quality crystalline TI thin films with surface-dominated conduction $[11,39,40]$. The first phase of this work was dedicated to designing an ultra-high vacuum chamber for thin films growth.

Molecular Beam Epitaxy (MBE) was developed in the late 1960s at Bell Labs by J.R. Arthur and A.Y. Cho [41, 42]. During MBE growth, elemental or compound materials are generally evaporated from sources contained in electrically heated furnaces or crucibles. The evaporated species pass through an ultra-high vacuum (UHV) environment and are deposited on a substrate that is hundreds of millimeter away from the evaporated materials. The temperature of the sources is set according to the type of material used since different materials have different melting points and vapor pressure. The UHV conditions as well as the absence of carrier gases result in high purity thin films. One way to realize MBE deposition is to employ an electron-beam evaporator (e-beam evaporator). Figure 2.3(a) shows the inside view of the evaporation chamber, illustrating the mutual positions of e-beam evaporator copper head $(A)$ and the substrate onto the heater (B). The substrate is at $\sim 10 \mathrm{~cm}$ from the evaporating source point (C). The evaporant beam 
Page 26 Chapter 2. Growth and surface morphology of $\mathrm{Bi}_{2} \mathrm{Te}_{3}$ topological insulator thin films

(a)

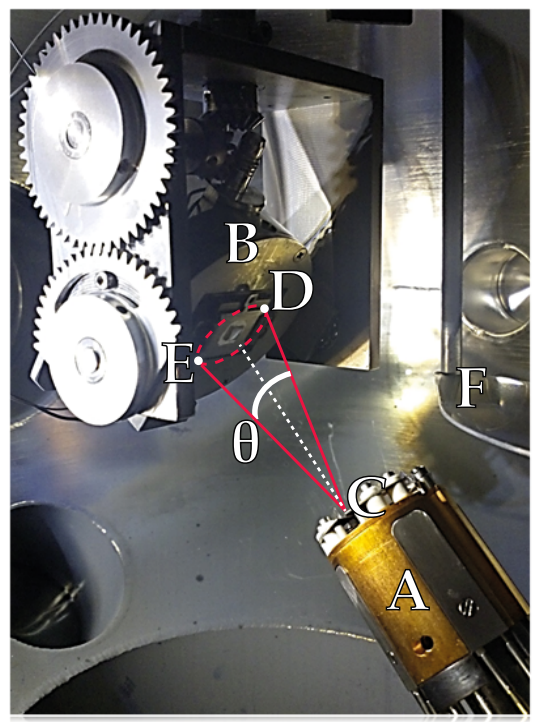

(b)

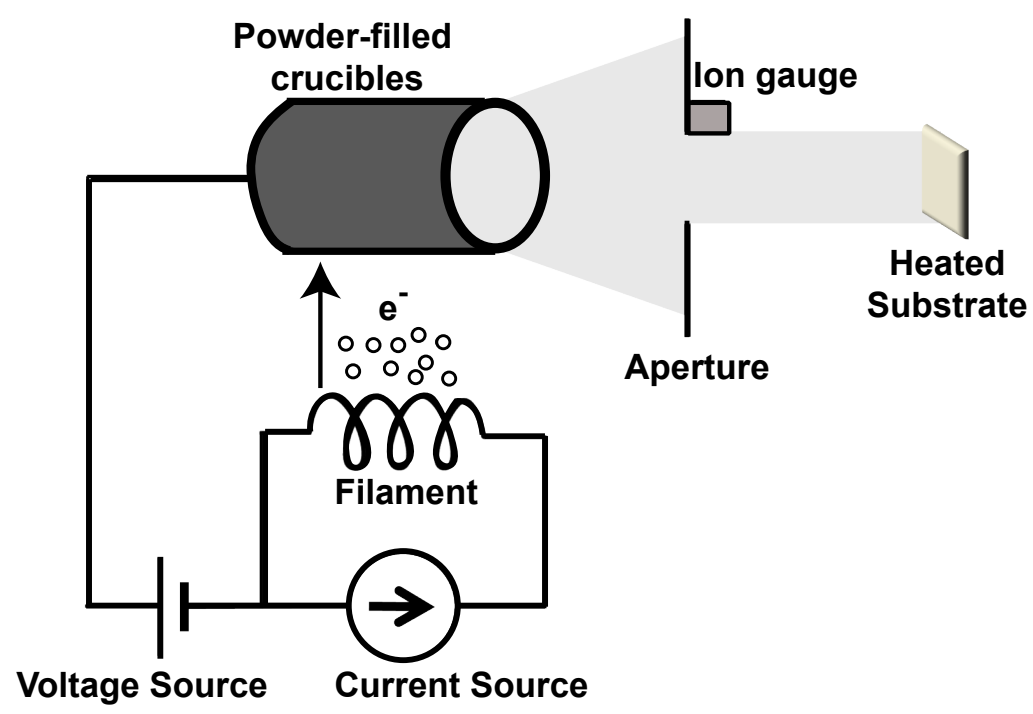

Figure 2.3: Electron beam evaporator. (a) Photograph of the inside view of the evaporation chamber. (b) Schematic illustration of the basic principle of an electron-beam evaporation process.

can cover an area of $2 \times 2 \mathrm{~cm}^{2}$ (dotted red line connecting points $\mathrm{D}$ and $\mathrm{E}$ ) with a uniformity better than $\sim 98 \%$ [43]. The central deposition profile of the source is identical to the $\cos ^{2} \theta$ distribution of a point source (C) [43]. The substrate shutter (F) can be used to shield the sample from the evaporant. Only $5 \times 5 \mathrm{~mm}^{2}$ and $10 \times 10 \mathrm{~mm}^{2}$ substrates were used to grow $\mathrm{TI}$ films. We used the EGCO4, Oxford applied research mini e-beam evaporator of $200 \mathrm{~W}$ e-beam power, equipped with 4 separate pockets sources of $0.2 \mathrm{~cm}^{3}$ assembled in the main copper body. Each pocket has its own dedicated filament that generate electron beam [44]. Pockets were loaded with powder materials of either $\mathrm{Bi}$, Te or Sb (Alfa Aesar of purity 99.999\%) inside molybdenum crucibles of size $393 \mathrm{~mm}^{3}$. This allows simultaneous co-evaporation of two or more materials onto the substrate.

Figure 2.3(b) illustrates the working principle of an electron-beam evaporator. The source material is heated by highly energetic electrons emitted by a filament and accelerated by the high voltage applied between the filament and the crucible $(2 \mathrm{kV})$. As the temperature rises, the source material starts to evaporate and the aperture shapes a well-defined beam of molecules leaving the evaporator. By controlling the filament current for the different crucibles, each crucible can be heated according to the type of material used, and its desired evaporation rate. The mini e-beam evaporator is equipped with an ion flux gauge that measures the ion current in the beam using flux monitoring plates in a feedback loop, so that one can regulate the power applied to the 
evaporant. Once calibrated in this way, it is possible to determine the deposition rate without using a thin film thickness monitor during growth. All $\mathrm{Bi}_{2} \mathrm{Te}_{3}$ thin films presented in this work were grown by co-evaporating high purity $\mathrm{Te}$ and $\mathrm{Bi}$ elements.

\subsubsection{In-situ X-ray photoemission spectroscopy}

Surface elemental composition, stoichiometry, and contamination effects were investigated using X-ray photoemission spectroscopy (XPS). The XPS technique is based on the detection of the photoelectrons when relatively high photon energies, ranging from $100 \mathrm{eV}$ to $2000 \mathrm{eV}$, are used to probe core electrons of the atoms comprising the solids. The XPS system is connected to the growth chamber via a distribution chamber (see Fig. 2.2). This was advantageous since XPS scan were being acquired right after growth without breaking the UHV conditions.

We used an omicron nanotechnology surface analysis system equipped with a monochromatic aluminium source $\left(K_{\alpha}\right.$ X-ray source XM1000) of a kinetic energy of $1486.7 \mathrm{eV}$. The kinetic energy of electrons emitted from the sample were analysed using a 7 channel EA 125 (energy analyser) operated in constant analyser energy (CAE) scan mode [45]. The EA 125 features an energy resolution better than $10 \mathrm{meV}$ (full width half-maximum: FWHM) and the resolution obtained in the CAE scan mode is constant throughout the whole kinetic energy range. The EA, in the CAE scan mode, scans the kinetic energy of the $\mathrm{X}$-ray generated electrons from the sample due to the photoelectric effect $[46,47]$.

The XPS technique is a surface sensitive technique because of the small escape depth of the electrons. The mean free path of the unscattered electrons is very small ( $5 \mathrm{~nm}$ for kinetic energies in the $1000 \mathrm{eV}$ ranges) [48]. This implies that an important fraction of the photoemission intensity is representing only the topmost surface layer. The inelastically scattered electrons, often called secondary electrons, are the most important factors that limit the electron escape depth [49]. This is mainly due to electron-electron and electron-phonon interactions. The secondary electrons give rise to an inelastic background that is present in almost all photoemission experiments, which typically decreases as kinetic energy increases. The background contains also information about the sample.

For the analysis of the photoemission spectra (e.g: determination of the surface stoichiometry), it is necessary to carefully select the background type and peak lineshape functions in order to obtain quantitative information from measurements. There exist numerous backgrounds that can be used in the peak fitting of XPS data. Nevertheless, each of the available backgrounds has its specific drawbacks and potency. The commonly used ones are the basic linear and the Shirley 
Page 28 Chapter 2. Growth and surface morphology of $\mathrm{Bi}_{2} \mathrm{Te}_{3}$ topological insulator thin films

backgrounds. Both of these background are purely mathematical and have no physical origin. We used the Shirley background to fit the XPS lineshape [50]. The Shirley background was introduced in lineshape fittings in order to take into account the asymmetry at the low kinetic energy side of the photoemission peaks. This asymmetry is due to the inelastic scattering that the photoelectrons experience before leaving the sample [50]. To fit the lineshape peak, we used a Voigt function [51]; which is a combination of a Gaussian and Lorentzian lineshapes. In the Voigt functional form, the Gaussian contribution takes into account of the line broadening due to the experimental resolution, while the Lorentzian contribution is due to the lifetime of the core excited hole. The Voigt profile is given by [51]:

$$
V(x ; \sigma, \gamma)=\int_{-\infty}^{\infty} G\left(x^{\prime} ; \sigma\right) L\left(x-x^{\prime} ; \gamma\right) \mathrm{d} x^{\prime}
$$

where $x$ is the energy difference from the line center, $G\left(x^{\prime} ; \sigma\right)$ the centred Gaussian profile

$$
G\left(x^{\prime} ; \sigma\right)=\frac{\mathrm{e}^{-x^{\prime 2} /\left(2 \sigma^{2}\right)}}{\sigma \sqrt{2 \pi}} ;
$$

and $L\left(x-x^{\prime} ; \gamma\right)$ the centred Lorentzian profile given by:

$$
L(x ; \gamma)=\frac{\gamma}{\pi\left(x^{2}+\gamma^{2}\right)} .
$$

For the determination of the surface stoichiometry, after background subtraction, and for fitting the area under the Te and Bi peaks, the atomic ratio was calculated using the expression [28, 52]:

$$
\frac{n_{T e}}{n_{B i}}=\frac{I_{T e} / I_{B i}}{S_{T e} / S_{B i}}
$$

where $n_{T e}$ and $n_{B i}$ are atomic density of Te and $\mathrm{Bi}$, respectively. $I_{T e}$ and $I_{B i}$ are the integrated Te and $\mathrm{Bi}$ peak intensity, respectively. $S$ is the atomic sensitivity factor, which depend on the X-ray source and the element of interest; it converts the relative peak areas to the relative numbers of atoms in the detected area $^{1}$ [52].

\subsubsection{Vacuum suitcase}

The low temperature scanning tunnelling microscopy (STM) and angle resolved photoemission spectroscopy (ARPES) systems that we used, are not connected to the COMAT setup. To transport thin films to these systems, we used a vacuum suitcase. The transport of samples in UHV conditions avoids contamination.

\footnotetext{
${ }^{1}$ For the fitting of the XPS peaks presented in section 4.2 of chapter 4 , we used these atomic sensitivity factor $S$ of $S_{3 d_{5 / 2}}^{T e}=5.4, S_{4 f_{7 / 2}}^{B i}=4.25, S_{4 f_{5 / 2}}^{B i}=3.4$ [28].
} 


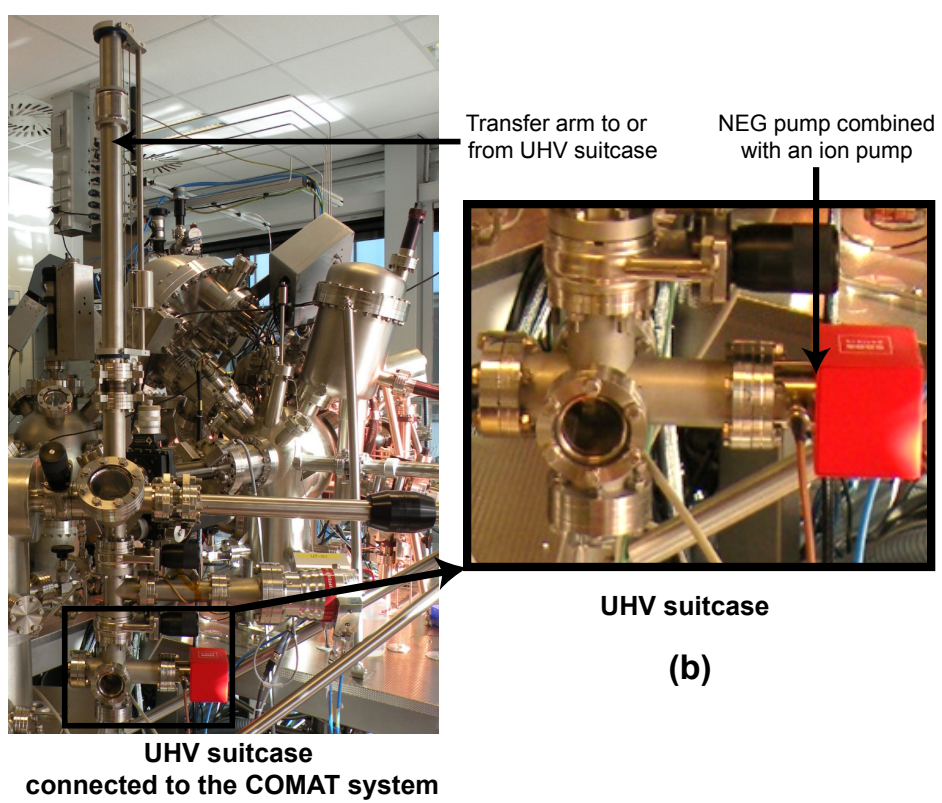

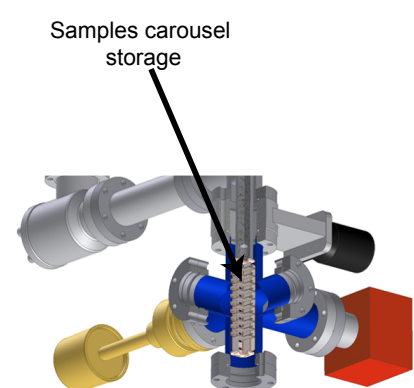

Inside view UHV suitcase

(c)

(a)

Figure 2.4: In-situ transport of samples. (a) The UHV suitcase connected to the combined system for thin films growth and in-situ characterization (b) Picture of the vacuum suitcase. (c) Schematic illustration of the inside view of the UHV suitcase.

Figure 2.4(a) shows the UHV suitcase connected to the COMAT system. After growth and insitu XPS characterizations, samples can be transferred to the UHV suitcase via the DC chamber using a magnetic transfer stick together with a vertical transfer arm. Figure 2.4(b) displays the vacuum case layout, consisting of a 6 -ways cross with pumps installed onto one single flange. This vacuum suitcase is a compact system with the capability to keep UHV conditions for weeks with no power during transportation [53]. The combination of the nonevaporable getter (NEG) pump ${ }^{2}$ with an ion pump allows the pumping of the entire spectrum of gases [31]. The hydrogen is very efficiently pumped by the NEG element ( $100 \mathrm{I} / \mathrm{s}$ pumping speed for $\mathrm{H}_{2}$ and $\mathrm{H}_{2} \mathrm{O}$ ), as well as all the other chemically active gases; whereas the argon and methane, not pumped by the NEG alone, are well pumped by the ion element [55]. This pumping capability helps in keeping the pressure lower than $2 \times 10^{-10}$ mbar throughout sample transfer and transportation. Inside the suitcase, samples are stored on a carousel which can contain up to 10 samples (see Fig. 2.4(c)). A detailed review on the description and working principle of compact non-evaporable getter and

\footnotetext{
${ }^{2}$ The pumping process is done by sorbing active gases in the vacuum chamber using a NEG material, which is based on the getter strip St707, produced by SAES getters [54]. The getter material contained in the pump cartridge does not need to be evaporated, it only needs to be reactivated (heated), under dynamic vacuum, to diffuse the passivation layer covering the surface of each getter particle into the bulk of the getter material. More than 100 reactivations are possible [55].
} 


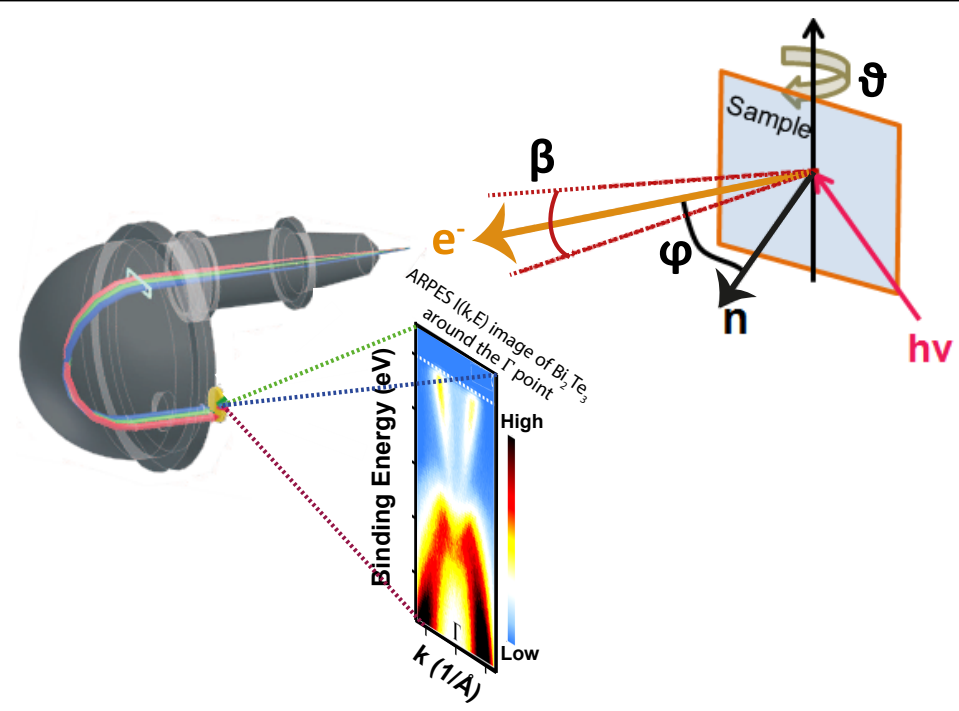

Figure 2.5: A schematic layout of an ARPES experimental geometry (adapted from Ref. [56]).

small ion pumps for in-situ sample transportation is given in references $[31,55,57,58]$

\subsubsection{In-situ angle resolved photoemission spectroscopy}

ARPES is one of the major surface-sensitive techniques employed to study the conducting surface states of Tls. We used in-situ ARPES to characterize and study the novel properties of $\mathrm{Bi}_{2} \mathrm{Te}_{3}$ thin films. Here, we introduce briefly the basic working principle of this technique.

ARPES is a refinement of the photoemission spectroscopy technique. It is the most direct method that measures simultaneously the kinetic energy and the angular distribution (or momentum) of the photoemitted electrons from a sample illuminated by light [49]. Figure 2.5 gives an illustration of the geometry of an ARPES experiment. From measured ARPES spectra, one obtains information on both the energy and momentum of the electrons inside the crystal, necessary for getting a detailed understanding of the electronic structure of materials. The main objective of an ARPES experiment is to determine the band dispersion relations $E(\mathbf{k})$ for the electrons in the solid using the kinetic energy and momentum of the measured photoelectrons in vacuum [49]. In practice, in order to get information on electrons inside the solid, one first measures both kinetic energy $E_{k i n}$ and momentum $\mathbf{K}$ of the photoelectrons in vacuum, that are collected by an energy analyser. From these values, it is then possible to gain information on binding energy $E_{B}$ and the momentum $\mathbf{k}$, of the electrons inside the solid using equations (3) in Ref. [49]. Upon shining photons $(h \nu)$ on the sample, the emission direction of the photoelectrons is specified by the polar $\varphi$ angle (emission angle) and azimuthal $\vartheta$ (manipulator angle). The vector $n$ is the sample surface normal and the angle $\beta$ is the analyser acceptance angle of the outgoing photoelectrons in momentum space. Often in an ARPES experiment, 2D colour maps are used because they 
represent data more conveniently (see Fig. 2.5). The acquired 2D colour map represents photoelectron intensity $\mathrm{I}\left(\mathrm{E}_{k i n}, \varphi, \vartheta\right)$ as a function of kinetic energy $\mathrm{E}_{k i n}$ and emission angle $\varphi$ along a particular direction given by $\vartheta$.

We acquired ARPES spectra in the vicinity of the Fermi level of $\mathrm{Bi}_{2} \mathrm{Te}_{3}$ thin films grown on different substrates. This was done in order to reveal the topological Dirac surface states and to confirm the absence of bulk states at the Fermi energy. The spectra were acquired both at low $(\sim 17 \mathrm{~K})$ and room temperature using a Scienta VUV5000 helium photon source of $21.2 \mathrm{eV}$ photon energy (He I line) and $40.8 \mathrm{eV}$ ( He II line) for valence band spectra. A Scienta 2002 hemispherical electron analyser was employed for 2D colour map acquisition.

\subsubsection{In-situ scanning tunnelling microscopy}

STM played a key role in the discovery of TI materials. For example, it was used to visualize the 2D Dirac surface states and to demonstrate some of the novel properties of TI materials, such as the absence of backscattering from non-magnetic impurities and a high transmission of TI surface states through barriers that reflect or absorb conventional surface states $[59,60]$. We used STM to probe the Dirac surface states of $\mathrm{Bi}_{2} \mathrm{Te}_{3}$ thin films.

Figure 2.6 illustrates the STM working principle. STM is an instrument that functions based on the principle of electronic tunnelling in order to image the surface of (conducting) samples at the atomic level. An atomically sharp tip (mostly chemically etched tungsten) is scanned over the surface of the sample at a distance of $10^{-9} \mathrm{~m}$. Roughly, the tunnelling current $I$ depends exponentially on the distance $(Z)$ between the STM tip and the sample surface; and it can be described by [61]:

$$
I \propto \frac{V}{Z} \exp (-2 \kappa Z)
$$

where $V$ is the applied voltage between the STM tip and the sample surface, and $\kappa$ the decay length. By applying a constant voltage between the STM tip and the sample surface, a tunneling current will start to flow. The size of this current depends on the bias voltage, the tip-sample separation, the lateral position of the tip with respect to the sample and the local density of states (LDOS). The atomic corrugation of the surface gives rise to variations in the tunnelling current with distance $Z$ between the sample and the tip. Thus, scanning the tip across the surface while using a feedback loop (in the z-direction) to keep this tunnelling current constant, the surface topographic images of the sample can be traced. This fulfils the role of microscopy of this instrument.

Alongside the surface topographic imaging, STM is widely used for spectroscopy. For the scanning 
Page 32 Chapter 2. Growth and surface morphology of $\mathrm{Bi}_{2} \mathrm{Te}_{3}$ topological insulator thin films

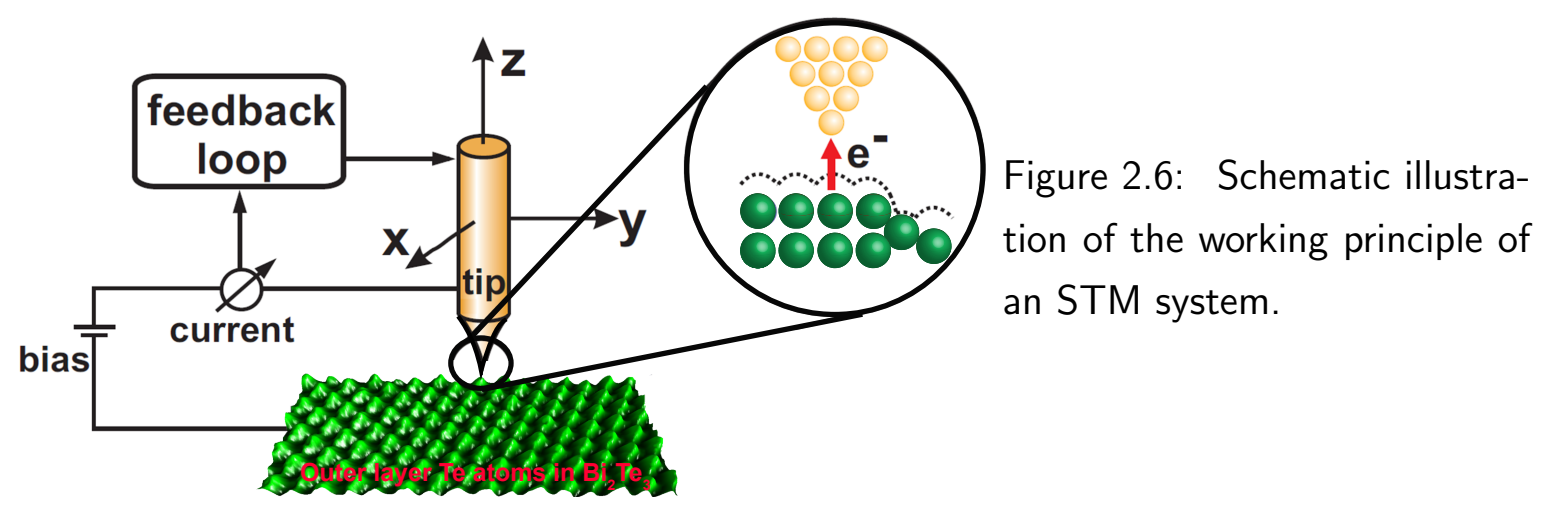

tunnelling spectroscopy (STS) measurement method, the STM tip is first brought above the desired position on the sample surface. Then, the tunnelling current is measured either in currentdistance spectroscopy mode $(\mathrm{I}(Z)$ as function of the tip-sample separation), in current-time spectroscopy mode $(\mathrm{I}(t))$ or as function of the bias voltage $(\mathrm{I}(V)$ : current-voltage mode). While the $\mathrm{I}(Z)$ spectroscopy is useful for the determination of the work function of the material, the $\mathrm{I}(t)$ spectroscopy mode is advantageous for the investigation of dynamics events on the samples surface, whereas the current-voltage $\mathrm{I}(V)$ spectroscopy provide information over the electronic properties of the measured sample with STM.

The topography and spectroscopy measurements, presented later in this dissertation (chapter 4), were acquired using an Omicron single probe low temperature scanning tunnelling microscopy. To achieve a permanent pressure of $<10^{-11}$ mbar, the STM measuring chamber was equipped with an ion getter pump together with a titanium sublimation pump. The sample was cooled down to $77 \mathrm{~K}$ using liquid Nitrogen. We used STM to study the surface topography and adsorbate layers, after exposure to ambient conditions, with atomic resolution in real space. STS measurements contributed to the investigation of the Dirac surface states.

\subsection{Growth dynamics}

The MBE growth process of TI films is mainly affected by the atomic flux ratio of the elements, the UHV conditions in the growth environment and the substrate temperature. To achieve highquality TI films, we first focus on optimizing these growth conditions. 


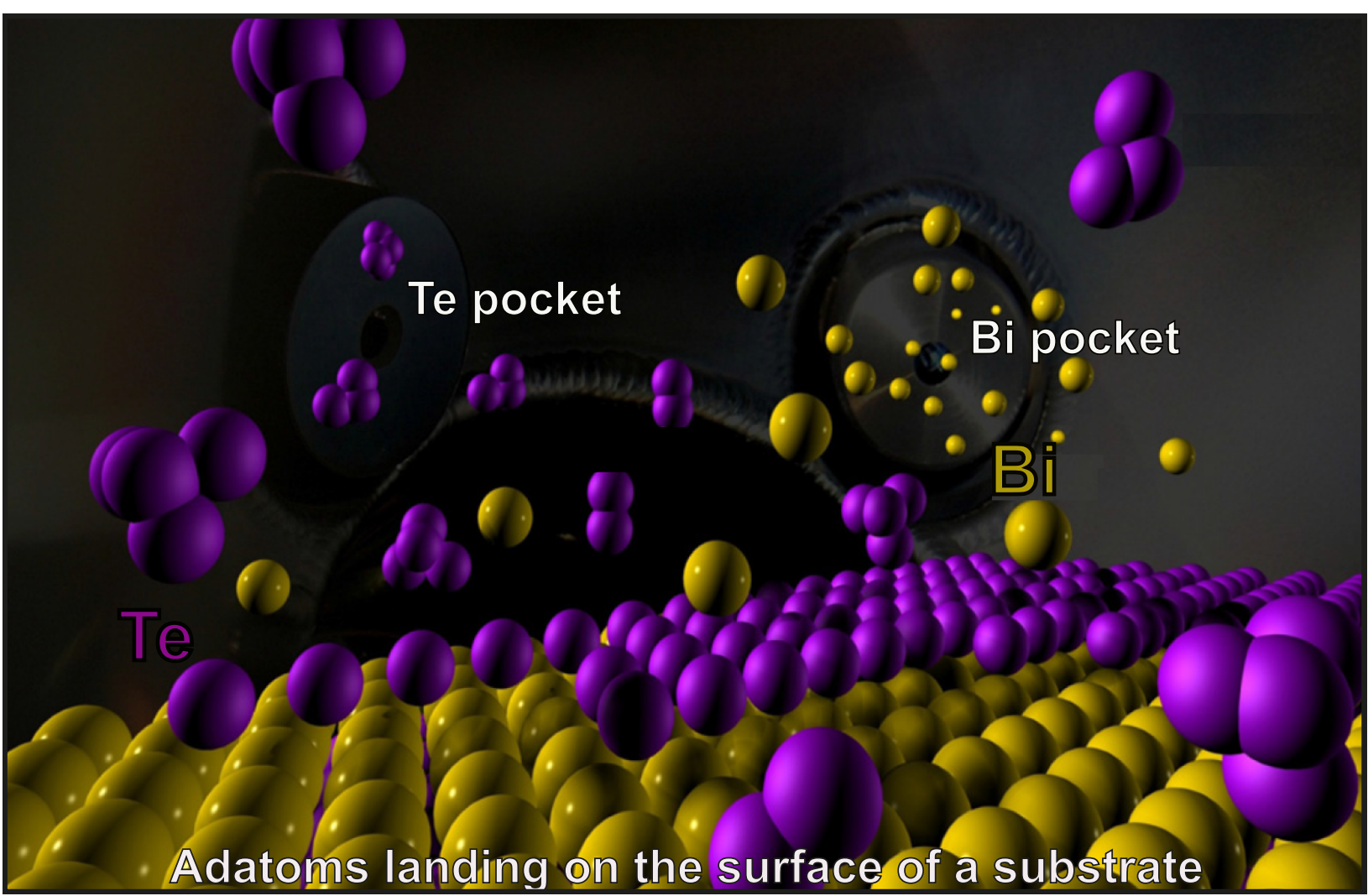

Figure 2.7: MBE growth dynamics. The Te-rich growth environment, which is the unique condition for obtaining the high-quality $\mathrm{Bi}_{2} \mathrm{Te}_{3}$ topological insulator thin films using standard MBE. Figure adapted from Ref. [62]

\subsubsection{Flux calibration and flux ratio optimization}

Figure 2.7 illustrates the Te-rich growth dynamics required for preparing $\mathrm{Bi}_{2} \mathrm{Te}_{3}$ films using $\mathrm{MBE}$ technique. For 3D TI growth, so as to achieve high-quality films, it is necessary to control the atomic flux ratio of the $\mathrm{V}(\mathrm{Bi}$ and $\mathrm{Sb})$ and $\mathrm{VI}(\mathrm{Se}$ and $\mathrm{Te})$ elements $[22,23]$. In the practice

\begin{tabular}{|c|l|l|l|l|l|}
\hline Material & $\begin{array}{l}\text { High volt- } \\
\text { age }(\mathbf{k V})\end{array}$ & $\begin{array}{l}\text { Filament cur- } \\
\text { rent }(\mathbf{A})\end{array}$ & $\begin{array}{l}\text { Emission cur- } \\
\text { rent } \mathbf{( m A )}\end{array}$ & $\begin{array}{l}\text { Typical } \\
\text { Flux (nA) }\end{array}$ & $\begin{array}{l}\text { Deposition } \\
\text { rate } \\
\mathbf{( A / s e c )}\end{array}$ \\
\hline $\mathrm{Bi}$ & 1.96 & 3.95 & 6.88 & 3.00 & 0.08 \\
\hline $\mathrm{Te}$ & 1.96 & 3.85 & 4.97 & 30.05 & 0.27 \\
\hline
\end{tabular}

Table 2.1: A summary of typical evaporator settings (high voltage, filament and emission currents), resulting flux and calibrated deposition rates used for $\mathrm{Bi}_{2} \mathrm{Te}_{3}$ thin films growth. 
Page 34 Chapter 2. Growth and surface morphology of $\mathrm{Bi}_{2} \mathrm{Te}_{3}$ topological insulator thin films
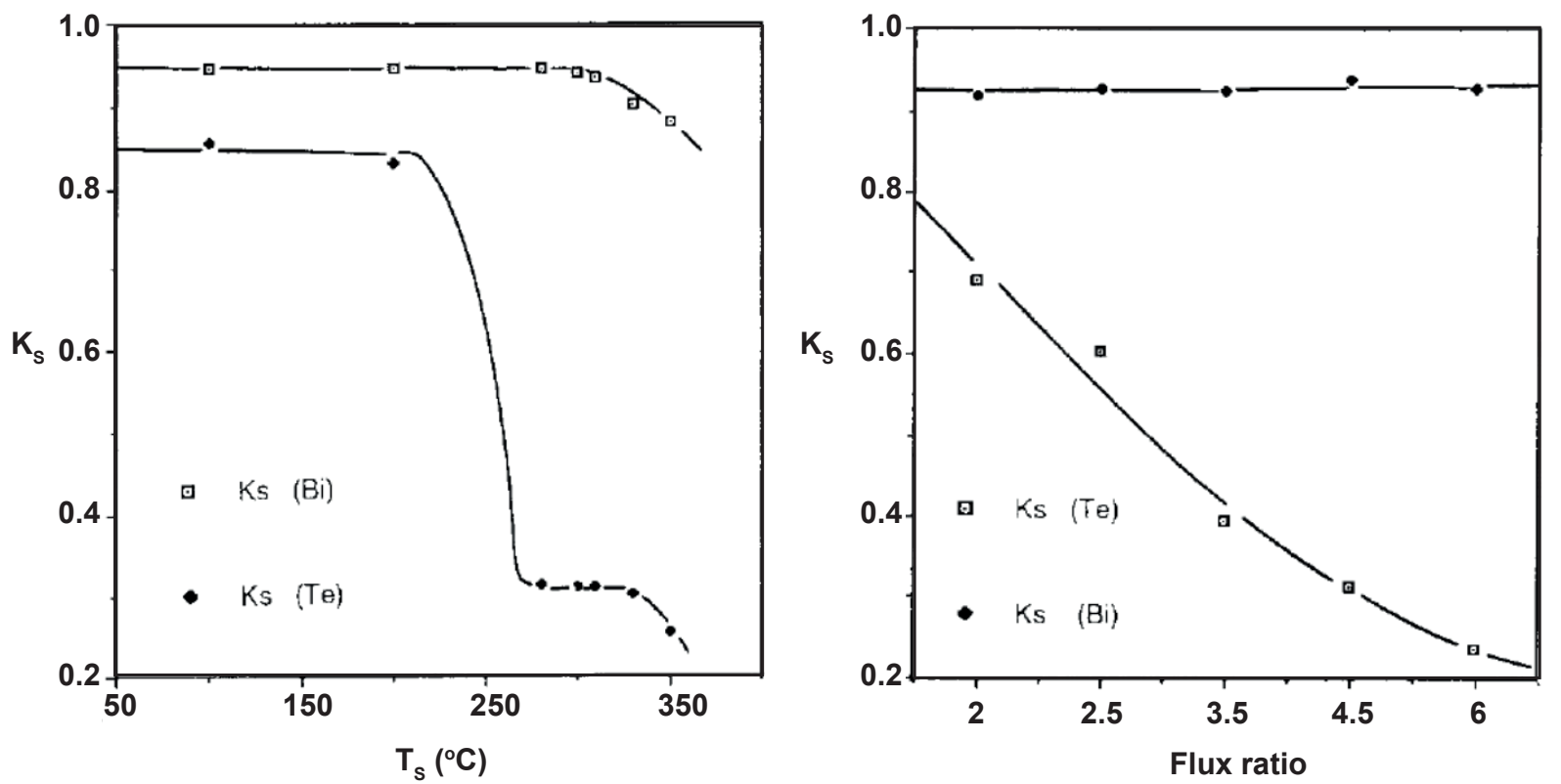

Figure 2.8: Sticking coefficient $\left(\mathrm{K}_{\mathrm{S}}\right)$ variation. (a) $\mathrm{K}_{\mathrm{S}}(\mathrm{Bi}, \mathrm{Te})$ as a function of substrate temperature at a flux ratio $(\mathrm{Te} / \mathrm{Bi})$ of 4.5 . (b) Variation of $\mathrm{K}_{\mathrm{S}}(\mathrm{Bi}, \mathrm{Te})$ as a function of flux ratio at a substrate temperature of $310^{\circ} \mathrm{C}$. Figure from Ref. [64].

of $\mathrm{Bi}_{2} \mathrm{Te}_{3}$ preparation, the growth should be performed in a Te-rich conditions in order to avoid $\mathrm{Te}$ vacancies and to achieve the right stoichiometry (a ratio of $\mathrm{Te}: \mathrm{Bi}=3: 2$ ) [63]. To obtain stoichiometric $\mathrm{Bi}_{2} \mathrm{Te}_{3}$ thin films, it is often suggested $[22,23,32]$ that the $\mathrm{Te} / \mathrm{Bi}$ atomic ratio should be in the range of 8 to 20 . Furthermore, when using thermal evaporation, the condition $\mathrm{T}_{\mathrm{Bi}}$ $>\mathrm{T}_{\text {Sub }}>\mathrm{T}_{\mathrm{Te}}\left(\mathrm{T}_{\mathrm{Bi}}, \mathrm{T}_{\text {Sub }}\right.$ and $\mathrm{T}_{\mathrm{Te}}$ are the Bi-cell, substrate and Te-cell temperatures, respectively) should be satisfied.

For the MBE growth using e-beam evaporators with different types of elements, one needs to control both the ratio of the elements and the rate of deposition on the substrate. The former is essential in order to achieve the correct stoichiometry while the latter determines the thickness of the film and influences the surface morphology. Thus, before initiating the growth of $\mathrm{Bi}_{2} \mathrm{Te}_{3}$ films, we performed a systematic atomic flux calibration so that the effective temperature of the $\mathrm{Te}$ and $\mathrm{Bi}$ crucibles should be set according to the desired rate of evaporation. Table 5.1 gives an overview of typical evaporation settings together with the resulting flux and deposition rate used in the growth of $\mathrm{Bi}_{2} \mathrm{Te}_{3}$ thin films. We performed a set of growth tests under different $\mathrm{Te} / \mathrm{Bi}$ flux ratios from which the optimal growth was found at the $\mathrm{Te} / \mathrm{Bi}$ beam flux ratio of 10 . Under the optimal substrate temperatures (discussed later) the growth rate was found to depend solely on the Bi flux, as also pointed out in previous studies [32]. This is because, contrary to the sticking coefficient of $\mathrm{Bi}$, the sticking coefficient of Te sharply drops as the substrate temperature is 
increased above $200^{\circ} \mathrm{C}$, as depicted in Fig. 2.8(a). In addition, the sticking coefficient of Te have been observed to decrease as the flux ratio $(\mathrm{Te} / \mathrm{Bi})$ is increased whereas the sticking coefficient of $\mathrm{Bi}$ is insensitive to changes in flux ratio (see Fig. 2.8(b)). The implication is that as long as $\mathrm{T}_{\text {sub }}$ is greater than the effective evaporation temperature of $\mathrm{Te}$ (around $220{ }^{\circ} \mathrm{C}$ in UHV condition), the Te molecular beam will not stick to the substrate without the simultaneous supply of Bi. These constraints set the lower limit to the growth temperature of stoichiometric deposition.

\subsubsection{Surface passivation: Two-step growth}

In section 2.1, we mentioned a variety of substrates used for Bi-based TI thin film growth. These substrates can be classified into ones with saturated bonds of the surface atoms, like graphene and hydrogen-terminated $\mathrm{Si}$, which are known to be Van der Waals type [23]. The other category is of those with dangling bonds like clean $\mathrm{Al}_{2} \mathrm{O}_{3}$ [0001], $\mathrm{InP}$ [111] and $\mathrm{SrTiO}_{3}$ [111], identified as non-Van der Waals type [23]. Because of the Van der Waals epitaxy [2, 3], the substrates are required to have an atom-terminated surface without dangling bonds. The dangling bonds on a clean surface of the substrate complicates adequate growth since they might lead to strong chemical interaction between the substrate and the TI deposit.

For the purpose of passivating the substrate surface and achieving atomically sharp interfaces between the TI film and the substrate, we used two-step growth scheme for the Van der Waals epitaxy of $\mathrm{Bi}_{2} \mathrm{Te}_{3}$. After initial treatments of the substrate, as described in section 2.1, we deposited a few nanometer ( 3 to $7 \mathrm{~nm}$ ) of $\mathrm{Bi}_{2} \mathrm{Te}_{3}$ at a slightly lower substrate temperature. This initial low-temperature layer, referred to as the nucleation layer, effectively fulfills the objective of passivating the substrate dangling bonds for the subsequent high-temperature Van der Waals epitaxy. This layer is then annealed as the substrate temperature is increased at higher growth temperatures and the second layer is epitaxially deposited on top. This two-step growth procedure resulted in $\mathrm{Bi}_{2} \mathrm{Te}_{3}$ thin films of high quality terrace-step surface morphology with less defects and large triangular mounds (Fig. 2.9 and Fig. 2.10).

\subsubsection{Substrate temperature}

For practical purposes, large terraces are preferred and the size strongly depends on the substrate temperature during growth, which needs to be chosen properly. To establish the correct substrate temperature $\left(T_{\text {sub }}\right)$ for the MBE growth of the $\mathrm{Bi}_{2} \mathrm{Te}_{3} \mathrm{TI}$ films, we performed a set of depositions at different $\mathrm{T}_{\text {sub }}$ for samples grown on both sapphire and STO substrates. These experiments showed that the optimal growth temperature of $\mathrm{Bi}_{2} \mathrm{Te}_{3}$ prepared on sapphire was at $170^{\circ} \mathrm{C}$ for 
Page 36 Chapter 2. Growth and surface morphology of $\mathrm{Bi}_{2} \mathrm{Te}_{3}$ topological insulator thin films

(a)

$170{ }^{\circ} \mathrm{C}$

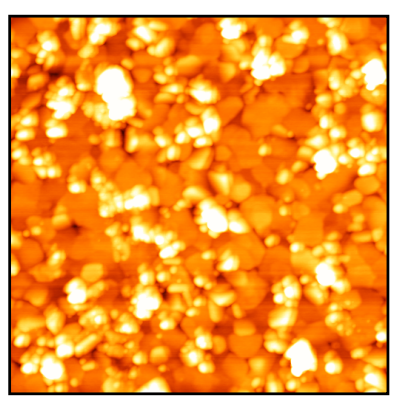

$3 \times 3 \mu m^{2}$ (b)

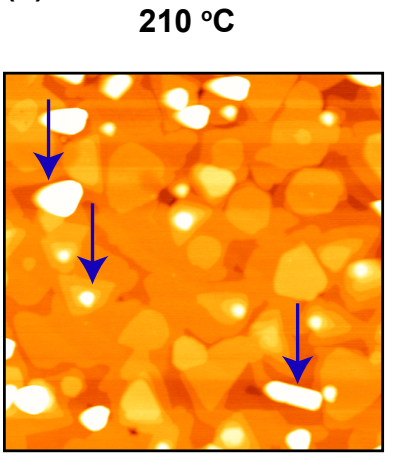

$3 \times 3 \mu m^{2}$ (c)

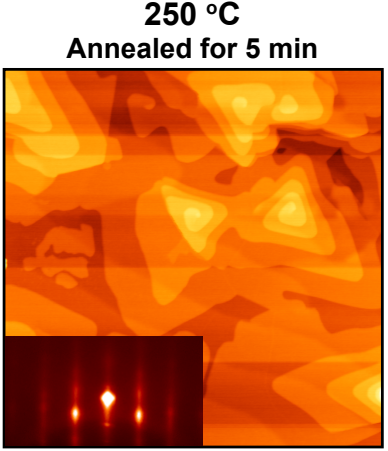

$3 \times 3 \mu m^{2}$ (d)

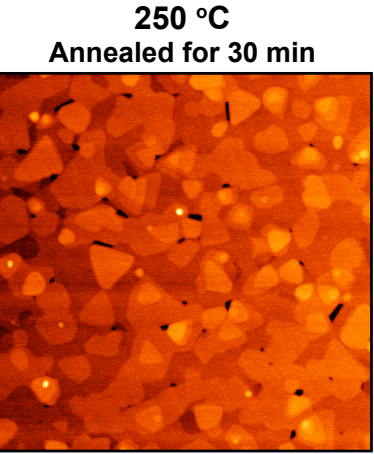

$3 \times 3 \mu m^{2}$

$15 \mathrm{~nm} \mathrm{Bi}_{2} \mathrm{Te}_{3}$ on $\mathrm{SrTiO}_{3}[111]$

Figure 2.9: Surface morphology at different substrate temperatures. The optimized growth temperature was found to be around $250{ }^{\circ} \mathrm{C}$. (b) The three blue arrows point to small and larger $3 \mathrm{D}$ defects, and quasi 1D nanostructures present at the surface. (c) The inset show the RHEED pattern of the surface of the sample taken after growth at room temperature.

the nucleation layer and $230^{\circ} \mathrm{C}$ for the subsequent layer. For samples grown on STO using the two-step method, the optimal temperature for the nucleation layer was also $170^{\circ} \mathrm{C}$, whereas the second layer was grown at a substrate temperature of $250^{\circ} \mathrm{C}$. Figure 2.9 demonstrates the improvement of the surface morphology as $T_{\text {sub }}$ is increased for four samples (prepared under the same conditions) of $15 \mathrm{~nm} \mathrm{Bi}_{2} \mathrm{Te}_{3}$ grown on STO [111].

In practice, at too low $\mathrm{T}_{\text {sub }}$, an amorphous or a polycrystalline films will be formed, as observed in the film grown at $170^{\circ} \mathrm{C}$ (Fig. 2.9(a)). This is because the adatoms will not have enough energy to diffuse and find the lowest potential energy site. As the substrate temperature is slightly, increased from $170^{\circ} \mathrm{C}$ to $210^{\circ} \mathrm{C}$, we observed an improved morphology with the appearance of triangular mound (Fig. 2.9(b)). However, at this temperature, there are still notable small and large either 3D or 1D defects, indicated by blue arrows in Fig. 2.9(b). These features were previously reported in Ref. [16]. For the sample grown at $250^{\circ} \mathrm{C}$ and annealed for $5 \mathrm{~min}$ in $\mathrm{Te}$ flux (after growth of the second layer), the 3D and 1D defects disappear and the surface is dominated by triangular mounds as depicted in Fig. 2.9(c). The corresponding reflection high energy electron diffraction (RHEED) image (inset Fig. 2.9(c)), taken after cooling down to room temperature, signals that the film is highly oriented. Annealing samples longer $(\sim 30 \mathrm{~min})$ helps in obtaining a smooth surface as observed in Fig. 2.9(d). However, it should be noted that this longer annealing step sometime resulted in re-evaporation of Te, consequently in Te deficient films or non-stoichiometric samples. This is probably due to the low sticking coefficient of Te on different substrates at high temperature (see Fig. 2.8) [64]. 
The substrate temperature also controls the growth kinetics during the MBE process via surface diffusion. The incident atoms from target materials will lose their momentum perpendicular to the substrate surface when they hit the substrate. But also, their in-plane diffusion motion will receive kinetic energy from the substrate, which depends on the substrate temperature. The diffusion of the adatoms onto the substrate can be described by a two-dimensional "random walk" model so that the mean displacement $\left(L_{M}\right)$ of diffusion will be given by:

$$
L_{M}=\alpha e^{\left(E_{\mathrm{DE}}-E_{\mathrm{DI}}\right) / 2 k_{B} T},
$$

where $E_{\mathrm{DE}}$ and $E_{\mathrm{DI}}$ are activation energies for the surface desorption and diffusion, respectively. $\alpha$ is the adsorption site spacing, determined by the lattice of the surface; $k_{B}$ the Boltzmann constant and $T$ the substrate temperature. From equation 2.3 , it is clear that the substrate temperature will play a key role in the surface diffusion process. Thus, the need to choose the optimal substrate temperature for the TI films growth carefully in order to maintain the sufficient diffusion on the surface, which leads to an improvement of the surface smoothness. The growth performed at too high temperature will mainly depend on the sticking coefficient of the material element and the films may not grow, because of a low sticking coefficient of the component on different substrates [64]. On the other hand, at low temperature, there will be randomly incident atoms frozen on the $\mathrm{Tl}$ surface, resulting in a kinetic roughness on the growth front (Fig. 2.9(a)).

\subsection{Surface morphology of $\mathrm{Bi}_{2} \mathrm{Te}_{3}$ thin films}

Subsequent to the MBE growth of $\mathrm{Bi}_{2} \mathrm{Te}_{3} \mathrm{TI}$ thin films, the surface morphology of the resulting films was investigated ex-situ using AFM. The morphology of the resulting films at optimal substrate temperatures demonstrated characteristic triangular-shaped terraces and steps as illustrated in Fig. 2.10(a) and Fig. 2.10(b). The triangular islands highlight the fcc stacking for the rhombohedral crystal structure of $\mathrm{Bi}_{2} \mathrm{Te}_{3}[16,65]$. The lateral size of these triangular mounds is $\sim 1.5 \mu \mathrm{m}$ for films grown both on sapphire and STO. The adjacent terraces were separated by an average step height of $\sim 1 \mathrm{~nm}$, consistent with the thickness of a single quintuple layer in the [001] direction of $\mathrm{Bi}_{2} \mathrm{Te}_{3}$ (see line profile in Fig. 2.10(a) and Fig. 2.10(b)) [66]. From the surface morphology point of view on a small scale, namely the lateral size of triangular mounds and the step height of terraces, the difference in growth on the two substrates is subtle.

The formation of spiral-like structures is observed at the top of the triangular island in the AFM images (see Fig. 2.10(a) and Fig. 2.10(b)). Previous studies on binary $\left(\mathrm{Bi}_{2} \mathrm{Te}_{3}\right.$ and $\left.\mathrm{Bi}_{2} \mathrm{Se}_{3}\right)$ and (off-) stoichiometric ternary Bi-based $\mathrm{TI}$ thin films have shown formation of spirals for samples grown on a variety of substrates $[8,10,13,22,67,69,70]$. The spirals are often considered 
Page 38 Chapter 2. Growth and surface morphology of $\mathrm{Bi}_{2} \mathrm{Te}_{3}$ topological insulator thin films

(a)

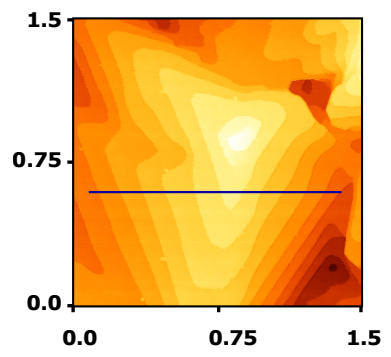

$70 \mathrm{~nm} \mathrm{Bi}_{2} \mathrm{Te}_{3}$ on $\mathrm{Al}_{2} \mathrm{O}_{3}[0001]$

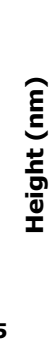

(b)

$20 \mathrm{~nm} \mathrm{Bi}{ }_{2} \mathrm{Te}_{3}$ on $\mathrm{SrTiO}_{3}[111]$
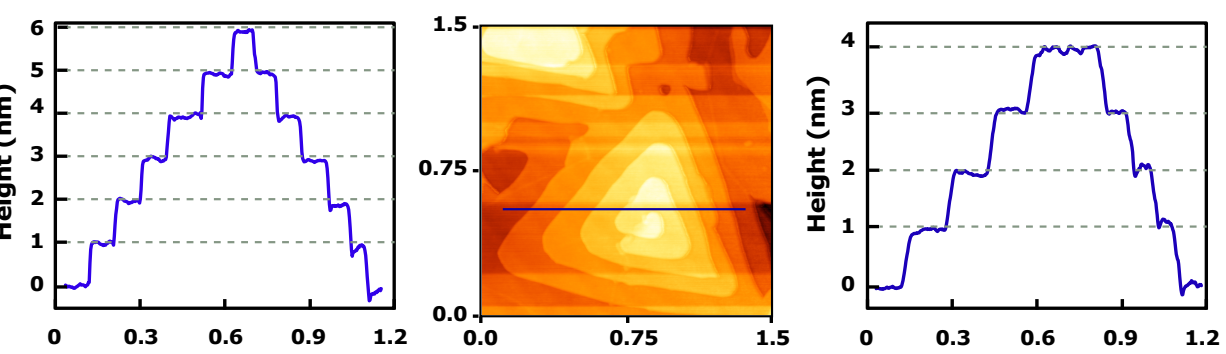

Distance $(\boldsymbol{\mu m})$

(c)

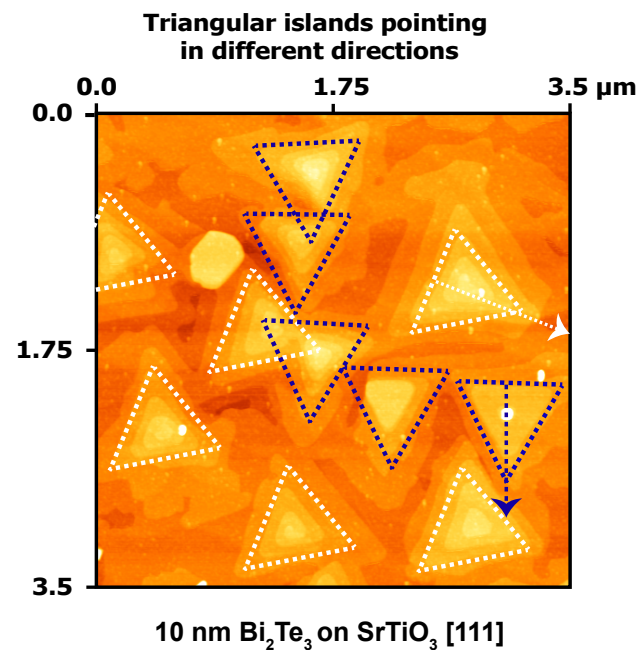

(d)

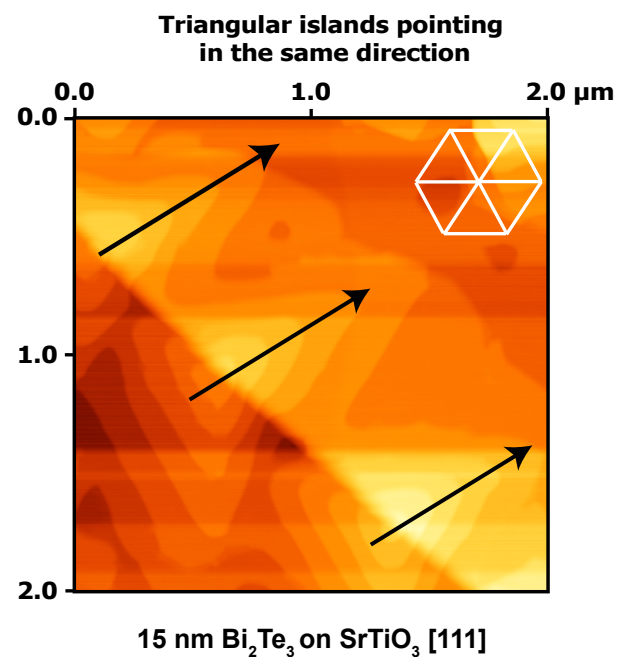

Figure 2.10: Growth morphology of $\mathrm{Bi}_{2} \mathrm{Te}_{3}$ films of different thickness. Atomic force microscopy images showing the surface morphology of $\mathrm{Bi}_{2} \mathrm{Te}_{3}$ thin films grown on (a) $\mathrm{Al}_{2} \mathrm{O}_{3}$ [0001] at the optimal substrate temperature of $230^{\circ} \mathrm{C}$ and (b) on $\mathrm{SrTiO}_{3}$ [111] at the optimal substrate temperature of $250^{\circ} \mathrm{C}$. From the line profile obtained across a series of wide steps (blue line), the step height was determined to be $1 \mathrm{~nm}$, for samples grown both on sapphire and STO. (c) Pyramids with different pointing directions, as illustrated by the blue and white triangles. The arrow indicate the in-plane orientation of the triangular mounds (d) Pyramids pointing in the same direction and having the same rotational directions. The black arrows indicate the pointing direction of the triangular islands. (Upper inset) The white hexagon at the top right corner illustrates the hexagonal crystal structure of $\mathrm{Bi}_{2} \mathrm{Te}_{3}$.

to come from misfit dislocations at the interface during heteroepitaxial growth; and when the dislocations have a screw component, they can emerge at the surface to form additional steps in order to facilitate the growth of spirals $[71,72,73,74,75]$. However, as it has been demonstrated 
in a recent study on spiral growth without dislocations of TI films [67], for Bi-based TI samples, where the adjacent QLs are only weakly bonded by Van der Waals forces, the epilayer is not expected to strongly bond to any substrate, i.e., no formation of dislocations is anticipated to seed the spiral growth. Thus, contrary to dislocation-mediated spiral growth, the observed spirals in $\mathrm{Bi}_{2} \mathrm{Te}_{3}$ thin films most likely form as a result of the pinning of the $2 \mathrm{D}$ growth fronts at the substrate steps or domain boundaries at the initial and intermediate stages of growth, respectively [67].

Figures 2.10 (c) and 2.10(d) show the surface morphology on a large scale (up to $3.5 \mu \mathrm{m}$ lateral size) for two films (10 and $15 \mathrm{~nm}$ ) grown on STO [111] using the two step procedure. The morphology reveals again predominantly triangular mounds confirming the hexagonal structure of the films. The blue and white triangles in Fig. 2.10(c) illustrate the in-plane orientation of the triangular mounds, and we see an in-plane misalignment of the islands which is often observed $[22,23]$. The islands on the substrates can also be grown to point in the same direction as indicated by black arrows in Fig. 2.10(d). Interestingly, these three triangular islands are also observed to climb over a straight terrace step of the STO substrate, while maintaining their straight edges. This morphology does not have an effect on the island coalescence. We will discuss electronic transport results, for thin films exhibiting this morphology, in the next chapter. 


\section{Bibliography}

[1] A. Koma, J. Cryst. Growth 201, 236 (1999).

[2] A. Koma, Surf. Sci. 26729 (1992).

[3] A. Koma, Thin Solid Films 216, 72 (1992).

[4] X. F. Kou, L. He, F. X. Xiu, M. R Lang, Z. M Liao, Y. Wang, A. V Fedorov, X. X. Yu, J. S. Tang, G. Huang, X. W. Jiang, J. F. Zhu, J. Zou and K. L. Wang, Appl. Phys. Lett. 98, $242102(2011)$.

[5] L. He, F. Xiu, X. Yu, M. Teague, W. Jiang, Y. Fan, X. Kou, M. Lang, Y. Wang, G. Huang, N. -C. Yeh and K. L. Wang, Nano Lett. 12, 1486 (2012).

[6] G. Zhang, H. Qin, J. Teng, J. Guo, Q. Guo, X. Dai, Z. Fang and K. Wu, Appl. Phys. Lett. 95, 053114 (2009).

[7] T. Hirahara, Y. Sakamoto, Y. Takeichi, H. Miyazaki, S. -I. Kimura, I. Matsuda, A. Kakizaki and S. Hasegawa, Phys. Rev. B 82, 155309 (2010).

[8] H. D. Li, Z. Y. Wang, X. Kan, X. Guo, H. T. He, Z. Wang, J. N. Wang, T. L. Wong, N. Wang and M. H. Xie, New J. Phys. 12, 103038 (2010).

[9] A. A. Taskin, S. Sasaki, K. Segawa and Y. Ando, Phys. Rev. Lett. 109, 066803 (2012).

[10] S. E. Harrison, S. Li, Y. Huo, B. Zhou, Y. L. Chen and J. S. Harris, Appl. Phys. Lett. 102, 171906 (2013).

[11] P. Ngabonziza, R. Heimbuch, N. de Jong, R. A. Klaassen, M. P. Stehno, M. Snelder, A. Solmaz, S. V. Ramankutty, E. Frantzeskakis, E. van Heumen, G. Koster, M. S. Golden, H. J. W. Zandvliet and A. Brinkman, Phys. Rev. B 92, 035405 (2015). 
[12] P. Ngabonziza, M. P. Stehno, H. Myoren, V. A. Neumann, G. Koster, and A. Brinkman, Adv. Electron. Mater. 2, 1600157 (2016).

[13] J. Chen, H. J. Qin , F. Yang, J. Liu, T. Guan, F. M. Qu , G. H. Zhang, J. R. Shi , X. C. Xie, C. L. Yang, K. H. Wu, Y. Q. Li and L. Lu, Phys. Rev. Lett. 105, 176602 (2010).

[14] G. Zhang, H. Qin, J. Chen, X. He, L. Lu, Y. Li and K. Wu, Adv. Funct. Mater. 21, 2351 (2011).

[15] A. Richardella, D. M. Zhang, J. S. Lee, A. Koser, D. W. Rench, A. L. Yeats, B. B. Buckley, D. D. Awschalom and N. Samarth, Appl. Phys. Lett. 97, 262104 (2010).

[16] Z. Y. Wang, H. D. Li, X. Guo, W. K. Ho and M. H. Xie, J. Cryst. Growth 334, 96 (2011).

[17] N. V. Tarakina, S. Schreyeck, T. Borzenko, C. Schumacher, G. Karczewski, K. Brunner, C. Gould, H. Buhmann and L. W. Molenkamp, Cryst. Growth Des. 12, 1913 (2012).

[18] S. Schreyeck, N. V. Tarakina, G. Karczewski, C. Schumacher, T. Borzenko, C. Brne, H. Buhmann, C. Gould, K. Brunner and L. W. Molenkamp, Appl. Phys. Lett. 102, 041914 (2013).

[19] X. Guo, Z. J. Xu, H. C. Liu, B. Zhao, X. Q. Dai, H. T. He, J. N. Wang, H. J. Liu, W. K. Ho and M. H. Xi, Appl. Phys. Lett. 102, 151604 (2013).

[20] C. -L. Song, Y. -L. Wang, Y. -P. Jiang, Y. Zhang, C. -Z. Chang, L. Wang, K. He, Xi Chen, J. -F. Jia, Y. Wang, Z. Fang, X. Dai, X. -C. Xie, X. -L. Qi, S. -C. Zhang, Q. -K. Xue and X. Ma, Appl. Phys. Lett. 97, 143118 (2010).

[21] L. Zhang, R. Hammond, M. Dolev, M. Liu, A. Palevski and A. Kapitulnik, Appl. Phys. Lett. 101, 153105 (2012).

[22] L. He, X. Kou and K. L. Wang, Phys. Status Solidi RRL 7, 50 (2013) and reference therein.

[23] M. -H. Xie , X. Guo, Z. -J. Xu and W. -K. Ho, Chin. Phys. B 22, 068101 (2013) and reference therein.

[24] X. He, T. Guan, X. Wang, B. Feng, P. Cheng, L. Chen, Y. Li and K. Wu, Appl. Phys. Lett. 101, 123111 (2012).

[25] J. Chang, Y. -S. Park and S. -K. Kim, Appl. Phys. Lett. 92, 152910 (2008).

[26] D. R. Lide, CRC Handbook of chemistry and physics, $96^{\text {th }}$ edition, CRC Press, Boca Raton, FL, 2005. 
[27] D. B. Chrisey (Editor) and G. K. Hubler (Editor), Pulsed laser deposition of thin films, $1^{\text {st }}$ Edition, John Wiley \& Sons (1994).

[28] J. F. Moulder, W. F. Stickle, P. E. Sobol and K. D. Bomben, Handbook of X-ray photoelectron spectroscopy, Physical Electronics, Inc., Minnesota, USA (1995).

[29] G. Binnig, H. Rohrer, Ch. Gerber and E. Weibel, Phys. Rev. Lett. 49, 57 (1982).

[30] G. Binnig and H. Rohrer, Rev. Mod. Phys. 59, 615 (1987).

[31] G. Firpo and A. Pozzo, Rev. Sci. Instrum. 75, 4828 (2004).

[32] Y. -Y. Li, G. Wang, X. Zhu, M. Liu, C. Ye, X. Chen, Y. Wang, K. He, L. Wang, X. Ma, H. Zhang, X. Dai, Z. Fang, X. Xie, Y. Liu, X. Qi, J. Jia, S. Zhang and Q. Xue, Adv. Funct. Mater. 22, 4002 (2010).

[33] G. Wang, X. Zhu, Y. Sun, Y. Li, T. Zhang, J. Wen, X. Chen, K. He, L. Wang, X. Ma, J. Jia, S. B. Zhang and Q. Xue, Adv. Funct. Mater. 23, 2929 (2011).

[34] H. Obara, S. Higomo, M. Ohta, A. Yamamoto, K. Ueno and T. lida, Jpn. J. Appl. Phys. 48, 085506 (2009).

[35] S. X. Zhang, L. Yan, J. Qi, M. Zhuo, Y. -Q. Wang, R. P. Prasankumar, Q. X. Jia, S. T. Picraux, Thin Solid Films 520, 6459 (2012).

[36] P. H. Le, W. -Y. Tzeng, H. -J. Chen, C. W. Luo, J. -Y. Lin and J. Leu, APL Mater. 2, 096105 (2014).

[37] R. Venkatasubramanian, T. Colpittsa, E. Watkoa, M. Lamvikb, N. El-Masryc, J. Crys. Grow. 170, 817 (1997).

[38] M. Ferhat, B. Liautarda, G. Bruna, J. C. Tedenaca, M. Nouaourab, L. Lassabatereb, J. Crys. Grow. 167, 122 (1996).

[39] K. Hoefer, C. Becker, D. Rata, J. Swanson, P. Thalmeier and L. H. Tjeng, Proc. Natl. Acad. Sci. USA 111, 14979 (2014).

[40] K. Hoefer, C. Becker, S. Wirth and L. H. Tjeng, AIP Adv. 5, 097139 (2015).

[41] A.Y. Cho., J. App. Phys. 41, 2780 (1970).

[42] J. R. Arthur, Surf. Sci. 43, 449 (1974). 
[43] Oxford Applied Reseach, General description of the Oxford applied research mini e-beam evaporators, (2015).

[44] Oxford Applied Research, Manual mini e-beam evaporator, EGCO4, Nursery Rd, Witney OX29 6SN, United Kingdom, (2015).

[45] Omicron Nanotechnology, Manual of the EA 125 Energy: User's, Version 2.1, Omicron Nanotechnology, (2002).

[46] H. R. Hertz, An. Phys. 267, 983 (1887).

[47] A. Einstein, An. Phys. 325, 199 (1906).

[48] M. P. Seah and W. A. Dench, Surf. Interface. Anal. 1, 2 (1979).

[49] A. Damascelli, Rev. Mod. Phys. 75, 2 (2003).

[50] D. A. Shirley, Phys. Rev. B 5, 4709 (1972).

[51] J. M. Luque, M. D. Calzada and M. Saez, J. Quant. Spectrosc. Radiat. Transfer 94, 151 (2005).

[52] M. P. Seah, I. S. Gilmore and S. J. Spencer, J. Electron. Spectrosc. Relat. Phenom. 120, 93 (2001).

[53] D. Sertore, P. Michelato, L. Monaco, P. Manini, F. Siviero, Proceedings of IPAC2011, San Sebastiàn, Spain (2011).

[54] SAES Getters S.p.A., Viale Italia, 77-20020 Lainate (Milano), Italy.

[55] Saes group, NEXTorr: The ideal pump for your portable vacuum case, (2016).

[56] P. Ngabonziza, Angle resolved photoemission spectroscopy study of the three-layered strontium ruthenate $\mathrm{Sr}_{4} \mathrm{Ru}_{3} \mathrm{O}_{10}$, MSc thesis University of Johannesburg, South Africa (2012).

[57] C. D. Park, S. M. Chung and P. Manini, J. Vac. Sci. Technol. A 29, 011012 (2011).

[58] D. R. Scherer, D. B. Fenner and J. M. Hensley, J. Vac. Sci. Technol. A 30, 061602 (2012).

[59] Z. Alpichshev, J. G. Analytis, J. -H. Chu, I. R. Fisher, Y. L. Chen, Z. X. Shen, A. Fang and A. Kapitulnik, Phys. Rev. Lett. 104, 016401 (2010).

[60] J. Seo, P. Roushan, H. Beidenkopf, Y. S. Hor, R. J. Cava and A. Yazdani. Nature 466, 343 (2010). 
[61] J. G. Simmons, J. Appl. Phys 34, 1793 (1963).

[62] M. Hanini (Editor), Molecular beam epitaxy: From Research to Mass Production, -p.587, Elsevier, Waltham (2013).

[63] J. Greenberg, Thermodynamic basis of crystal growth: P-T-X phase equilibrium and nonStoichiometry, Springer-Verlag, Berlin (2002).

[64] A. Mzerd, D. Sayah, J. C. Tedenac and A. Boyer, J. Cryst. Growth 140, 365 (1994).

[65] M. H. Xie, S. M. Seutter, W. K. Zhu, L. X. Zheng, H. Wu, S. Y. Tong, Phys. Rev. Lett. 82, 2749 (1999).

[66] H. Zhang, C. -X. Liu, X. -L. Qi, X. Dai, Z. Fang and S. -C. Zhang, Nat. Phys. 5, 438 (2009).

[67] Y. Liu, M. Weinert, and L. Li, Phys. Rev. Lett. 108, 115501 (2012).

[68] A. Richardella, D. M. Zhang, J. S. Lee, A. Koser, D. W. Rench, A. L. Yeats, B. B. Buckley, D. D. Awschalom and N. Samarth Appl. Phys. Lett. 97, 262104 (2010).

[69] M. Ferhat, J. C. Tedenac, and J. Nagao, J. Cryst. Growth 218, 250 (2000).

[70] F. Yang, A. A. Taskin, S. Sasaki, K. Segawa, Y. Ohno, K. Matsumoto, and Y. Ando, Appl. Phys. Lett. 104, 161614 (2014).

[71] W. K. Burton, N. Cabrera, and F. C. Frank, Philos. Trans. R. Soc. London A 243, 299 (1951).

[72] A. Karma and M. Plapp, Phys. Rev. Lett. 81, 4444 (1998).

[73] B. Heying, E. J.Tarsa, C. R. Elsass, P. Fini, S. P. DenBeaars, and J. S. Speck, J. Appl. Phys. 85, 6470 (1999).

[74] Y. Cui and L. Li, Phys. Rev. B 66, 155330 (2002).

[75] T. Akasaka, Y. Kobayashi, and M. Kasu, Appl. Phys. Lett. 97, 141902 (2010). 


\section{Magnetoresistance in $\mathrm{Bi}_{2} \mathrm{Te}_{3}$ topological insulator thin films}

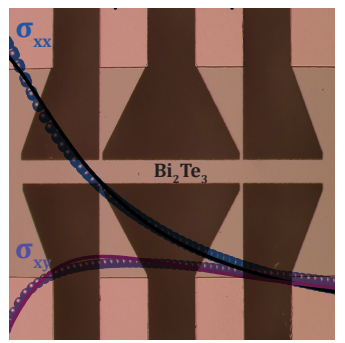

In the field of topological insulators (TIs), major emphasis is placed on investigating the TI surface states. However, there is a challenge in magnetotransport characterizations of $\mathrm{TI}$ materials, as it is necessary to carefully disentangle the electronic transport nature of the bulk from that of the surface. This is mainly because the charge transport is dominated by bulk conductivity due to residual carriers in the second generation $\mathrm{TI}$ materials $\left(\mathrm{Bi}_{2} \mathrm{Te}_{3}, \mathrm{Bi}_{2} \mathrm{Se}_{3}\right.$, and $\left.\mathrm{Sb}_{2} \mathrm{Te}_{3}\right)$, thus complicating the direct exploitation of the surface states in transport experiments. For $\mathrm{Bi}_{2} \mathrm{Te}_{3}$ samples, bulk single crystals and thin films exposed to air during fabrication processes are bulk conducting, with the chemical potential in the conduction band $[1,2]$. In this chapter, we report on the magnetoresistance of $70 \mathrm{~nm}$ thick $\mathrm{Bi}_{2} \mathrm{Te}_{3}$ films, which have been exposed to ambient conditions. The trends observed in magnetotransport reflect the topological nature of transport properties coming from bulk states.

Part of the work (section 3.2.3) discussed in this chapter was submitted for publication as: D.P. Leusink, R. G. J. Smits, P. Ngabonziza, X. L. Wang, S. Wiedmann, U. Zeitler and A. Brinkman, http://arxiv.org/abs/ 1412.4065 (2014). 


\subsection{Introduction}

The interest in nano-electronics arises from fascinating electrical transport effects that arise when the sample size $L$ is made smaller than some characteristic electronic length scales [3, 4]. One of these characteristic lengths is the carrier mean free path $l_{e}=v_{F} \tau_{e}$, which defines the average distance an electron can propagate between scattering events. Here, $\tau_{e}$ is a typical time scale electrons are scattered by impurities or phonons in a disordered system, whereas $v_{F}$ is the Fermi velocity. According to a quantum mechanical description, the phase of the electron wavefunction $\varphi$ changes after a certain time $\tau_{\varphi}$ (phase coherence time) due to various interactions, e.g inelastic scattering. The resulting phase coherence length $l_{\varphi}=v_{F} \tau_{\varphi}$ is the length scale on which quantum mechanical interference phenomena become relevant $[3,4]$. This quantity is, thus, crucial in understanding the crossover between quantum transport regime and classical effects. When an electron scatters elastically, also the spin direction has a finite lifetime to readjust, giving the characteristic spin-orbit length $l_{S O}[5]$. All three length scales are relevant in understanding the magnetotransport data presented below.

\subsection{Magnetotransport in $\mathrm{Bi}_{2} \mathrm{Te}_{3}$ thin films}

Studying transport properties in an externally applied magnetic field is one of the powerful probes for the investigation of the topological nature of the surface state. From magnetotransport experiments, interesting phenomena, like weak antilocalization $[6,7,8,9]$, nonlinear Hall effect $[10,11]$, Shubnikov-de-Haas $(\mathrm{SdH})$ oscillations $^{1}[10,12,13,14]$, and a large and sometime linear magnetoresistance (LMR) $[15,16,17,18]$, are often measured in TI materials. Here and in subsequent chapter (chapter 5), we report these phenomena in $\mathrm{Bi}_{2} \mathrm{Te}_{3}$ topological insulator thin films. The $\mathrm{Bi}_{2} \mathrm{Te}_{3}$ thin films were grown on $\mathrm{Al}_{2} \mathrm{O}_{3}$ [0001] substrates using molecular beam epitaxy (MBE) by co-evaporating high purity $\mathrm{Te}$ and $\mathrm{Bi}$ in a Te rich environment. Details on the growth procedure are discussed in chapter 2. A systematic in-situ characterization of films grown in the same conditions is presented in chapter 4 . The $70 \mathrm{~nm}$ films discussed in this chapter were structured ex-situ into Hall bars by means of optical lithography and Ar ion beam etching. Figure 3.1(a) shows an optical image of a typical Hall bar device measured in magnetotransport experiments. Both the sheet resistance and the Hall resistance were measured as function of the

\footnotetext{
${ }^{1}$ Other different types of quantum oscillations such as Aharonov-Bohm (AB) surface oscillations in nanowires, Altshuler-Aronov-Spivak (AAS) oscillations, and universal conductance fluctuations (UCF) have also been reported $[19,20,21]$.
} 
applied magnetic field in the temperature range from $300 \mathrm{~K}$ down to $2 \mathrm{~K}$. We used an excitation current of $1 \mu \mathrm{A}$ in the measurements.

\subsubsection{Weak antilocalization and non-linear Hall effect in $\mathrm{Bi}_{2} \mathbf{T e}_{3}$ thin films}

Experimentally, weak antilocalization (WAL) and nonlinear Hall effect are probably the most commonly observed effects in transport experiments on $\mathrm{TI}$ single crystals and thin films at low temperature $[6,7,8,9,10,11]$. First, we discuss the WAL effect. Even though the WAL effect ${ }^{2}$ is not exclusive to topological materials [22, 23, 24, 25], it is evidence of an important feature of the topological surface states, namely, the spin-momentum locking. The antilocalization is caused by the $\pi$-Berry phase picked up along two time-reversed self-intersecting paths. To elucidate this point, in the diffusive regime $\left(l_{e} \gg L\right)$ if the electron spin is strongly locked to its momentum and scatters around random paths to return to its initial position, then the spin direction would rotate as the momentum rotates around the loop. The spin of the electron will have rotated by $\pi$. If the same electron scatters along an opposite loop, its spin would have rotated by $-\pi$. In case that the electron travels around these loops before losing its phase information, then at the interference point, the net spin rotation would be $2 \pi$ (corresponds to a net phase rotation of $\pi$ ). The interference is thus destructive, the backscattering channel is suppressed and the total resistance is lowered when compared to the situation without spin-momentum locking [26]. In the WAL effect, an externally applied magnetic field destroys the interference effects by inducing a phase shift away from $\pi$ so that the backscattering is increased and the resistance thus increases toward its normal value. Thus, when the WAL is taking place in a system, application of an external magnetic field breaks the destructive interference and leads to a negative magnetoconductivity, i.e resistivity increase. In the absence of spin-momentum locking the total phase difference is $2 \pi$ and interference is constructive, leading to an enhancement of backscattering, which is known as weak localization (WL) $[22,27]$. This increases the probability of electrons to stay in one spatial location, which in result reduces their ability to transport currents. The WL effect will take place as long as $l_{S O}>l_{\varphi}>l_{e}$, whereas a crossover to the WAL behaviour is expected when $l_{S O}$ is reduced (increased spin orbit coupling) with $l_{S O}=l_{e}<l_{\varphi}$.

\footnotetext{
${ }^{2}$ This correction is called WAL because it is a weak correction to conductivity of the order of magnitude of the quantum of conductance $e^{2} / h$. Compared to the classical part, which is much larger in the mesoscopic regime of weak disorder, this correction is small. In addition, the WAL results in an increase of the conductance, implying that electrons will be less localized in the absence of an externally applied magnetic field compared to when a magnetic field is applied.
} 
(a)

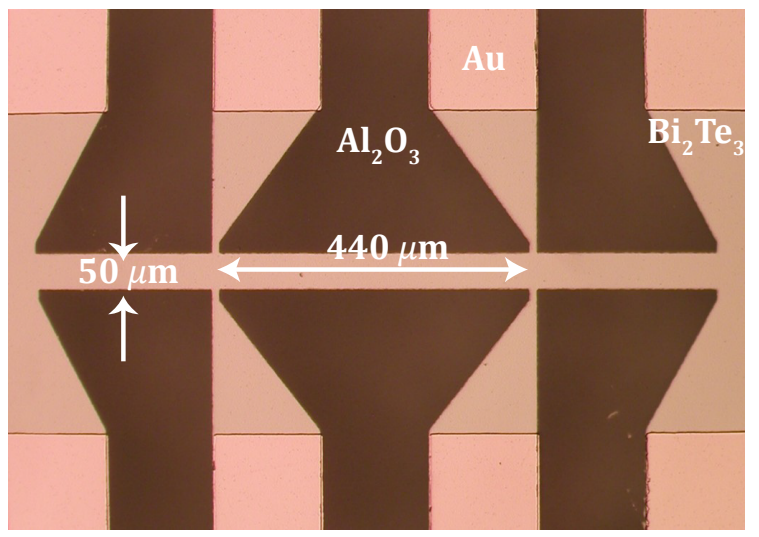

(b)

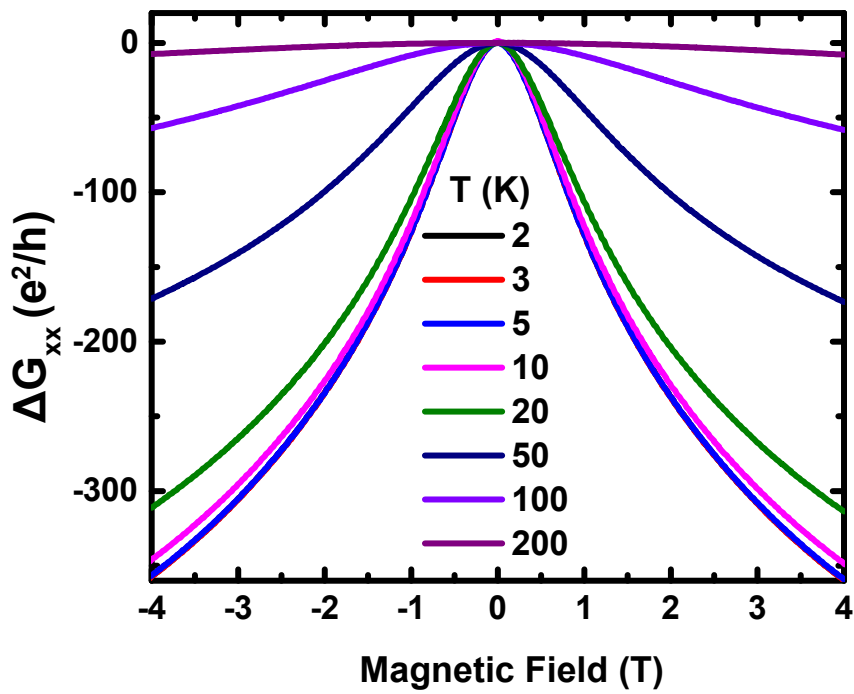

Figure 3.1: (a) A typical optical micrograph image of a structured Hall bar device from the $70 \mathrm{~nm}$ $\mathrm{Bi}_{2} \mathrm{Te}_{3}$ film grown on $\mathrm{Al}_{2} \mathrm{O}_{3}$ [0001]. Lateral dimensions of the Hall bar are indicated in the image. The ratio of length over width is: $L / W=8.8$ (b) Magnetoconductance $\Delta G_{x x}(B)$ measurements at various temperature for magnetic field applied perpendicularly to the film surface.

The full expression of the quantum correction to the conductivity in perpendicular magnetic field, given by the Hikami-Larkin-Nagaoka (HLN) model [22], is often used to described the WAL effect:

$$
\begin{aligned}
\Delta G(B)= & -\frac{e^{2}}{2 \pi h}\left[\Psi\left(\frac{B_{\varphi}}{B}+\frac{1}{2}\right)-\ln \left(\frac{B_{\varphi}}{B}\right)\right] \\
& -\frac{e^{2}}{\pi h}\left[\Psi\left(\frac{B_{S O}+B_{e}}{B}+\frac{1}{2}\right)-\ln \left(\frac{B_{S O}+B_{e}}{B}\right)\right] \\
& +\frac{3 e^{2}}{2 \pi h}\left[\Psi\left(\frac{(4 / 3) B_{S O}+B_{\varphi}}{B}+\frac{1}{2}\right)-\ln \left(\frac{(4 / 3) B_{S O}+B_{\varphi}}{B}\right)\right] ;
\end{aligned}
$$

where $\Psi$ is the digamma function, $B_{i}=\frac{\hbar}{4 e l_{i}}$ is the characteristic magnetic field corresponding to different length scales $(i=\varphi, S O$ and $e$ for the phase coherence, spin-orbit coupling and elastic scattering lengths, respectively). In systems with spin-momentum locked surface states, the $B_{S O} \sim 1 / L_{S O}^{2}$ is infinite, thus only one term in equation 3.1 survives. The quantum correction to the $2 \mathrm{D}$ conductivity due to WAL effect becomes:

$$
\Delta G(B)=\alpha \frac{e^{2}}{\pi h}\left[\Psi\left(\frac{B_{\varphi}}{B}+\frac{1}{2}\right)-\ln \left(\frac{B_{\varphi}}{B}\right)\right] .
$$

The pre-factor $\alpha$ accounts for the WAL contributions from different conducting channels. From the HLN equation, the pre-factor $\alpha$ is expected to be $-1 / 2$ for a single $2 \mathrm{D}$ conducting surface with strong spin-orbit coupling. Contributions from either a conducting bulk or disorder induced 
(a)

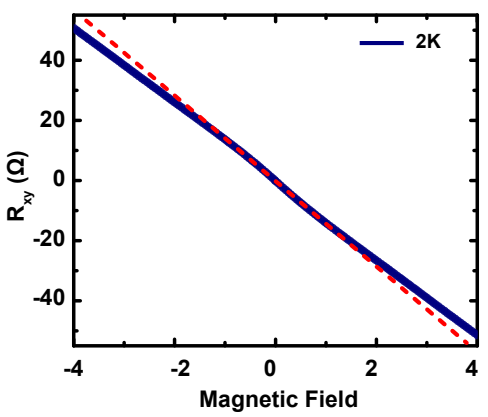

(b)

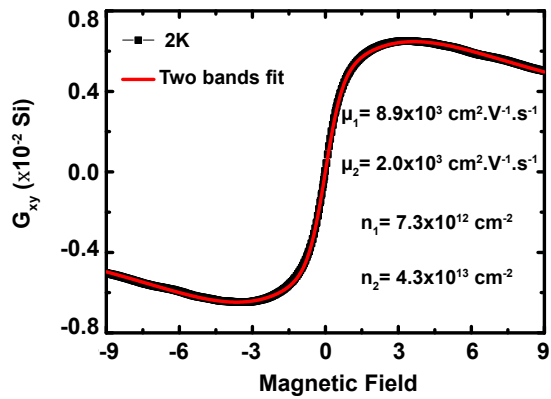

(c)

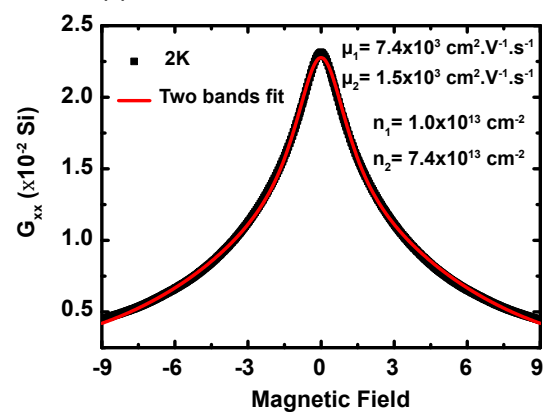

Figure 3.2: Nonlinear Hall effect in $\mathrm{Bi}_{2} \mathrm{Te}_{3}$ thin films grown on $\mathrm{Al}_{2} \mathrm{O}_{3}$ [0001]. (a) Hall resistance versus magnetic field at $2 \mathrm{~K}$ plotted together with a one-carrier model fitting in order to highlight the presence of multiple bands carrier transport in $R_{x y}(B)$. (b) and (c) Hall and longitudinal conductance data fitted independently with the two-carrier model using expressions 3.3 below.

electron-electron interactions in the system $[28,29]$ will drive the value of $\alpha$ to deviate from $-1 / 2$ towards more positive values. We will present in chapter 5 (section 5.3) an elaborated analysis on extracted values of $\alpha$ in capped thin films from the fits to the simplified HLN equation 3.2.

In Fig. 3.1(b), we plot the sheet magnetoconductance, $\Delta G_{x x}(B)=G_{x x}(B)-G_{x x}(B=0 T)$, as function of the applied perpendicular magnetic field for various temperatures. Here, the sheet conductance $G_{x x}$ is given by $\frac{R_{S}}{R_{S}^{2}+R_{x y}^{2}}$, with $R_{S}$ being the sheet resistance and $R_{x y}$ the measured Hall resistance. At low temperatures, $\Delta G_{x x}$ displays a sharp, cusp-like maximum at zero magnetic field, clearly evident at $2 \mathrm{~K}$. As the temperature increases, the $\Delta G_{x x}$ cusp at low B is broadened and, finally, disappears (above $20 \mathrm{~K})$. The measured $\Delta G_{x x}$ is much larger $\left(\sim 350 \frac{e^{2}}{h}\right.$ at $\left.4 \mathrm{~T}\right)$ than what would be expected for quantum correction to the conductivity (WAL or WL corrections). We attribute this behaviour to a multiband effect, as will be elaborated below.

Next, we discuss the nonlinear Hall effect in $\mathrm{Bi}_{2} \mathrm{Te}_{3}$ thin films. The nonlinearity in the Hall signal, $R_{x y}(B)$, have been measured in different TI materials and it was suggested to originate from the coexistence of bulk and surface transport channels [10, 11, 30, 31]. If all carriers participating in transport had the same mobility, $R_{x y}(B)$ would show linear behaviour with the slope determined by $1 /\left(e R_{H}\right)$, with $R_{H}$ being the Hall coefficient. However, when there are multiple types of carriers with different (but comparable) mobilities, nonlinearity shows up in the $R_{x y}(B)$ data. The nonlinearity in Fig. 3.2(a) suggests the presence of multiple carrier types. From the sign of the Hall signal, the majority charge carriers are found to be electrons. In applied perpendicular magnetic field, the diagonal and off-diagonal entries in the conductance tensor can be written as 
function of the carrier mobilities $\left(\mu_{1}, \mu_{2}\right)$ and the sheet carrier densities $\left(n_{1}, n_{2}\right)$ as [30, 31]:

$$
G_{x x}=e\left(\frac{n_{1} \mu_{1}}{1+\mu_{1}^{2} B^{2}}+\frac{n_{2} \mu_{2}}{1+\mu_{2}^{2} B^{2}}\right) ; G_{x y}=e B\left(\frac{n_{1} \mu_{1}^{2}}{1+\mu_{1}^{2} B^{2}}+\frac{n_{2} \mu_{2}^{2}}{1+\mu_{2}^{2} B^{2}}\right) ;
$$

where $e$ and $B$ are the electron charge and magnetic field, respectively. We apply this two-carrier model to the $70 \mathrm{~nm} \mathrm{Bi}_{2} \mathrm{Te}_{3}$ film and extract the transport parameters independently from $G_{x y}$ and $G_{x x}$. It turns out that it provides an excellent fit to our Hall resistance data as shown Fig. 3.2(b). From these fits, we extract the mobilities $\left(\mu_{1}, \mu_{2}\right)$ and carrier densities $\left(n_{1}, n_{2}\right)$ (see inset Fig. 3.2(b)). The obtained 2D carrier concentration $\left(n_{2}\right)$ is high compared to what would be expected for a $\mathrm{Bi}_{2} \mathrm{Te}_{3}$ sample with the Fermi level situated in the bulk band gap ${ }^{3}$; thus pointing to a bulk carrier contribution to transport. In Fig. 3.2(c), we plot the two carrier band fit to the longitudinal conductance $\left(G_{x x}\right)$. Unfortunately, a simultaneous fit of both the measured longitudinal and Hall conductivity was not possible within the framework of the standard twoband model. This disagreement is common in TI systems and it remains a pending issue [32]. In practice, the two-band model does not consider a B-field dependence of the scattering rates, even though the magnetoresistance $R_{x x}(B)$ is usually large and complicated in Tls. As a result, almost always the combination of parameters obtained from the two-band analyses of $R_{x y}(B)$ do not agree with the ones extracted from $R_{x x}(B)$. To address this problem, we propose an alternative model in subsection 3.2.3, which can be used to fit the longitudinal and transverse magnetoresistance data simultaneously [33].

\subsubsection{Shubnikov-de-Haas oscillations in $\mathrm{Bi}_{2} \mathrm{Te}_{3}$ thin films}

In Fig. 3.3(a), we present the magnetoresistance (MR) data for various temperatures. Here, MR is defined as $\mathrm{MR}=\frac{R(B)-R(B=0)}{R(B=0)}$. Between 1.5 and $5 \mathrm{~T}$, the $\mathrm{MR}$ is almost linear, but it starts to deviate from the linear behaviour at high magnetic field, where it shows a superposition of small oscillations together with a quasi-linear increase up to a magnetic field of $9 \mathrm{~T}$. The latter behaviour is often observed in TI samples $[15,16,17,18]$. In the semiclassical picture for two or more bands, the MR is quadratic and saturates when the carriers travel in closed orbits [34] with a MR of only a few percent. First, we discuss the quantum oscillations, then we will address the linear magnetoresistance effect in $\mathrm{Tls}$ in subsection 3.2.3.

Quantum oscillations ${ }^{4}$ such as the Shubnikov-de Haas $(\mathrm{SdH})$ oscillations are a powerful probe of

\footnotetext{
${ }^{3}$ For the Dirac surface states, the carrier density is in the $\sim 10^{12} \mathrm{~cm}^{-2}$ regime or lower $10^{13} \mathrm{~cm}^{-2}$ range. A detailed discussion on this point is presented in section 5.3 of chapter 5

${ }^{4}$ Quantum oscillations are determined by the Landau quantization of the energy levels of crystalline solids in magnetic fields due to the density of states (DOS) being periodically modulated as a function of magnetic field. In particular, the oscillations occurring in conductivity are called Shubnikov-de Haas oscillations [35].
} 
(a)

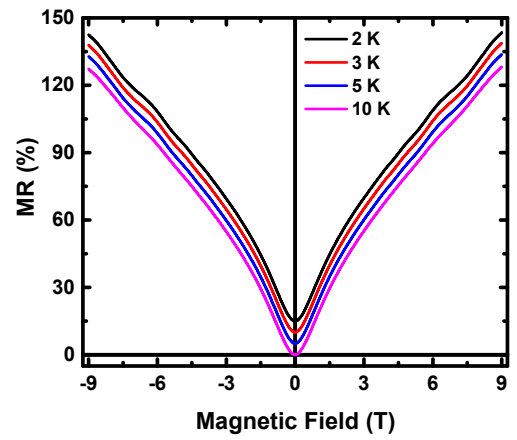

(c)

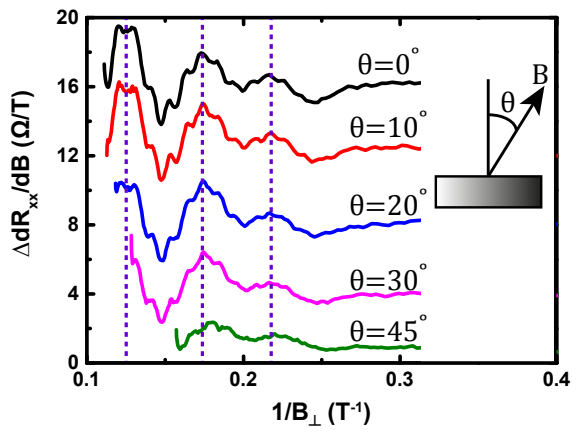

(e)

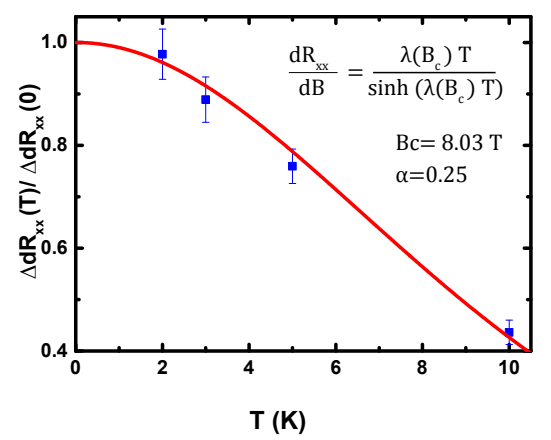

(b)

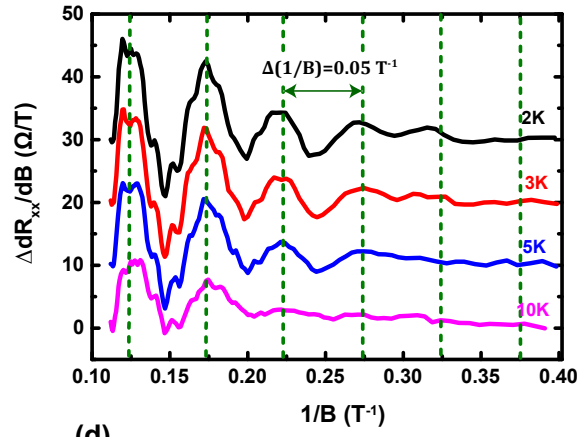

(d)

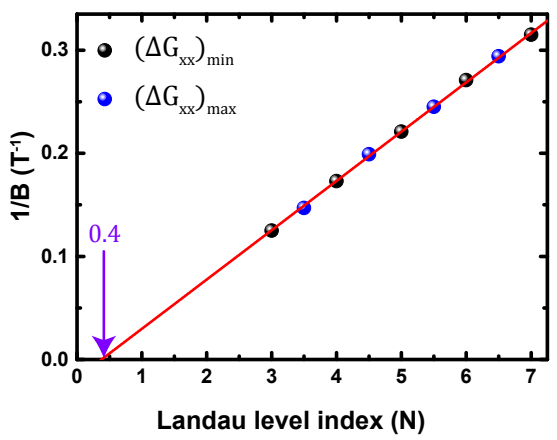

(f)

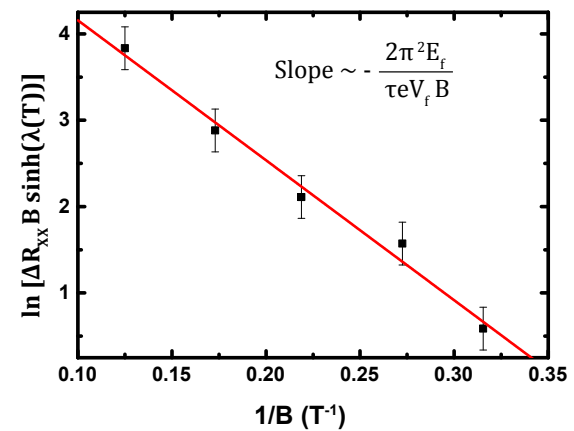

Figure 3.3: Magnetoresistance and Shubnikov-de-Haas oscillations in $\mathrm{Bi}_{2} \mathrm{Te}_{3}$ thin films grown on $\mathrm{Al}_{2} \mathrm{O}_{3}$ [0001]. (a) Magnetoresistance measured at various temperature. At high magnetic field (above $4.5 \mathrm{~T}$ ) small quantum oscillations are visible up to $10 \mathrm{~K}$. (b) After subtraction of a background, the oscillations become more visible. The extracted period of oscillations is $\Delta(1 / B)=0.05 \mathrm{~T}^{-1}$. (c) The $\mathrm{SdH}$ oscillations are plotted at different perpendicular component of applied magnetic field with $B_{\perp}=$ $B \cos (\theta)$ (see inset). For clarity, offsets are added in (b) and (c). (d) Landau-level fan diagram from $\mathrm{SdH}$ oscillations in (c). Linear fitting of the periodic maxima and minima as a function of the Landau level index $N$ gives a non-zero intersects at $\sim 0.4$. (e) Temperature dependence of the normalized longitudinal resistance. The solid red line is the best fit to $\lambda(T) / \sinh (\lambda(T)$ ). (f) The Dingle plot of $\ln \left[\Delta R_{x x} B \sinh (\lambda(T)]\right.$ versus $1 / B$ at $2 \mathrm{~K}$. The transport lifetime $\tau$, mean free path $l$ and mobility $\mu$ are calculated from the parameters obtained from the best fit to this expression. 
the nature of the metallic state in Tls $[7,10,15]$, and they have been previously interpreted either as surface or bulk oscillations in $\mathrm{TI}$ samples $[7,32,36]$. The $\mathrm{SdH}$ effect probes extrema in the cross section of the Fermi surface (FS). For example, their angular dependence in tilted magnetic fields can provide information about the size and the shape of the FS [36] and, more importantly, about the dimensionality of the FS $[7,37]$. The frequency of the magnetoresistance oscillation as a function of the inverse magnetic field is obtained from the Onsager relation [35]: $F=\frac{\hbar}{2 \pi e} A\left(\epsilon_{F}\right)$, where $A\left(\epsilon_{F}\right)$ is the maximal cross-sectional area of the FS in a plane perpendicular to the magnetic field. The full momentum dependence of the FS can be mapped by rotating the magnetic field with respect to the sample plane. The measured magnetic field-dependent longitudinal resistance $R_{x x}$ shows traces of $\mathrm{SdH}$ oscillations, which are also visible in the extracted MR data presented in Fig. 3.3(a) for four different temperatures. The oscillations survive up to $10 \mathrm{~K}$. The superimposed oscillations become clearly evident after the subtraction of a smooth polynomial background. The oscillatory part of the longitudinal resistance $\left(\Delta R_{x x}\right)$ displays periodic extrema with $1 / B$ (see Fig. 3.3(b)). For the SdH oscillations at $2 \mathrm{~K}$, the period of oscillations is $0.05 \mathrm{~T}^{-1}$. In the case of topological surface states with no spin degeneracy, the carrier density corresponding to this oscillation period would be $n=\frac{e}{h} \cdot \frac{1}{\Delta(1 / B)}=1.0 \times 10^{12} \mathrm{~cm}^{-2}$. Using the expression $n_{S d H}=\frac{k_{F}^{2}}{2 \pi}$, this carrier density would give a Fermi wave vector of $k_{F}=0.025 \AA^{-1}$. For a closed, spherical FS $\mathrm{FS}^{5}$, the $3 \mathrm{D}$ carrier density would then be $n_{S d H}^{3 D}=\frac{k_{F}^{3}}{3 \pi}=1.6 \times 10^{18} \mathrm{~cm}^{-3}$. On the other hand, if one considers a spin degenerate, cylindrical FS, the 3D carrier density is determined using the expression: $n_{S d H}^{3 D}=\frac{1}{c} n_{S d H}^{2 D}=1 \times 10^{19} \mathrm{~cm}^{-3}$, with $c$ being the $\mathrm{Bi}_{2} \mathrm{Te}_{3}$ lattice parameter. From the Hall coefficient $1 / e R_{H}=5.8 \times 10^{13} \mathrm{~cm}^{-3}$, we extract a carrier density of $n_{3 D}=n_{2 D} / d=8.2 \times 10^{18} \mathrm{~cm}^{-3}$, with $d=70 \mathrm{~nm}$ being the sample thickness. Since this carrier density is large, it is interpreted as bulk carriers. Furthermore, in comparison to the spherical and cylindrical FS carrier densities: $1.6 \times 10^{18} \mathrm{~cm}^{-3}<8.2 \times 10^{18} \mathrm{~cm}^{-3}<1 \times 10^{-19} \mathrm{~cm}^{-3}$, the data suggest that the FS would have a shape in between a spherical FS and open cylindrical FS, but more elongated towards a cylindrical one.This implies that the measured quantum oscillations would probably be coming from bulk states, an observation that is in agreement with previous reports on $\mathrm{Bi}_{2} \mathrm{Se}_{3}$ samples $[36,38]$.

Figure 3.3(c) depicts the angle dependent $\mathrm{SdH}$ oscillations at $2 \mathrm{~K}$. From these data, we observe that periodic maxima and minima, are overlapping when plotted against the perpendicular component of the magnetic field $\left(B_{\perp}=B \cos \theta\right)$, as indicated by the dashed line in Fig. 3.3(c). This suggests a two-dimensional nature of the oscillating channels, consistent with the nearly cylindri-

\footnotetext{
${ }^{5}$ When the charge carriers get high, the FS may become anisotropic such that $k_{F}$ along the $k_{z}$ direction gets considerably larger than $k_{F}$ in the $\left(k_{x}, k_{y}\right)$ plane. Thus, the FS will shift from being a closed spherical FS at low carrier densities into an open cylinder-like FS at high carrier densities. A detailed discussion on the evolution of the FS is presented in Ref. [36].
} 
cal FS, rendering the conductance very anisotropic (but still bulk) [38]. There can also be other non-TI surface channels, like the trivial non-topological 2DEG states due to band-bending, which can give rise to 2D transport features [39]. In Fig. 3.3(d), we plot the $1 / B$ values corresponding to the maxima (blue circles) and the minima (black circles) of the oscillatory part of longitudinal conductance $\left(\Delta G_{x x}\right)$ versus Landau level index $(N)$ by assigning the index as discussed in Ref. $[12,32]$. A linear fit of the data yields a finite intercept at $N=0.4$, which is consistent with a half-filled lowest Landau level expected for systems with a Dirac cone or the $\pi$-Berry phase in the bulk conduction band [27].

Now, we analyse the mobilities extracted from both $\mathrm{SdH}$ oscillations and Hall signal. At $2 \mathrm{~K}$, the zero magnetic field Hall mobility (corresponding to the above mentioned Hall carrier density) is $\mu=\frac{1}{e R s n}=2800 \mathrm{~cm}^{2} \mathrm{~V}^{-1} \mathrm{~s}^{-1}$. The surface mobility is determined through the analysis of $\mathrm{SdH}$ oscillations at different temperatures. The temperature-dependent amplitude of the oscillatory contribution to the resistance of a two-dimensional system can be described with the LifshitzKosevich expression [40]:

$$
\Delta R \propto \sum_{i} e^{-\lambda_{i} T_{D_{i}}} \frac{\lambda_{i}(T)}{\sinh \left(\lambda_{i}(T)\right)} \sin \left(\frac{2 \pi f_{i}}{B}+\phi_{i}\right) ;
$$

where $T$ is the temperature, $f_{i}$ and $\phi_{i}$ are the frequency and phase of the oscillations, respectively. $\lambda_{i}(T)$ is given by the expression: $\lambda_{i}(T)=2 \pi^{2} k_{B} T m_{c y c l} /(\hbar e B)$, with $m_{c y c l}$, $\hbar$ and $k_{B}$ being the cyclotron mass, the reduced Planck constant and the Boltzmann constant, respectively. The Dingle temperature, which has information about the quantum mobility, is given by: $T_{D_{i}}=\frac{2 \pi^{2} E_{F}}{\tau e B v_{F}^{2}}$, where $E_{F}$ is the Fermi energy, $\tau$ the transport life time, $e$ the electron charge and $v_{F}$ the Fermi velocity. At a constant magnetic field of $B_{c}$, the Lifshitz-Kosevich expression 3.4 is simplified to:

$$
\Delta R \propto \sum_{i} \frac{\alpha_{i}\left(B_{c}\right) T}{\sinh \left(\alpha_{i}\left(B_{c}\right) T\right)}
$$

with $\alpha_{i}=2 \pi^{2} k_{B} m_{c y c l} /\left(\hbar e B_{c}\right)$. Figure 3.3(e) gives the temperature dependence of the normalized longitudinal resistance oscillation amplitude extracted at a constant magnetic field $B_{c}=8.03 \mathrm{~T}$. Performing the best fit to expression 3.5 yields a value of $\alpha=0.25$ from which $m_{\text {cycl }}$ is extracted to be $0.133 m_{e}$ ( $m_{e}$ is the free electron mass). In order to estimate the transport lifetime of the surface states, we use the Dingle plot $[12,37,41]$ depicted in Fig. $3.3(\mathrm{f})$. From the slope of the Dingle plot, we can extract a transport lifetime $\tau=2.75 \times 10^{-13} \mathrm{~s}$, from which we derive the mean free path of $l=v_{F} \tau \simeq 150 \mathrm{~nm}$. Using the expression $\mu_{s}=e \tau / m_{c y c l}=e l^{S d H} / \hbar k_{F}$, the surface mobility is found to be $\mu_{s} \simeq 3600 \mathrm{~cm}^{2} \mathrm{~V}^{-1} \mathrm{~s}^{-1}$, which is close to the Hall mobility estimated above. Thus, based on the extracted mobilities (from SdH oscillations and Hall data), we further conclude that bulk states are significantly contributing to the measured $\mathrm{SdH}$ oscillations. 


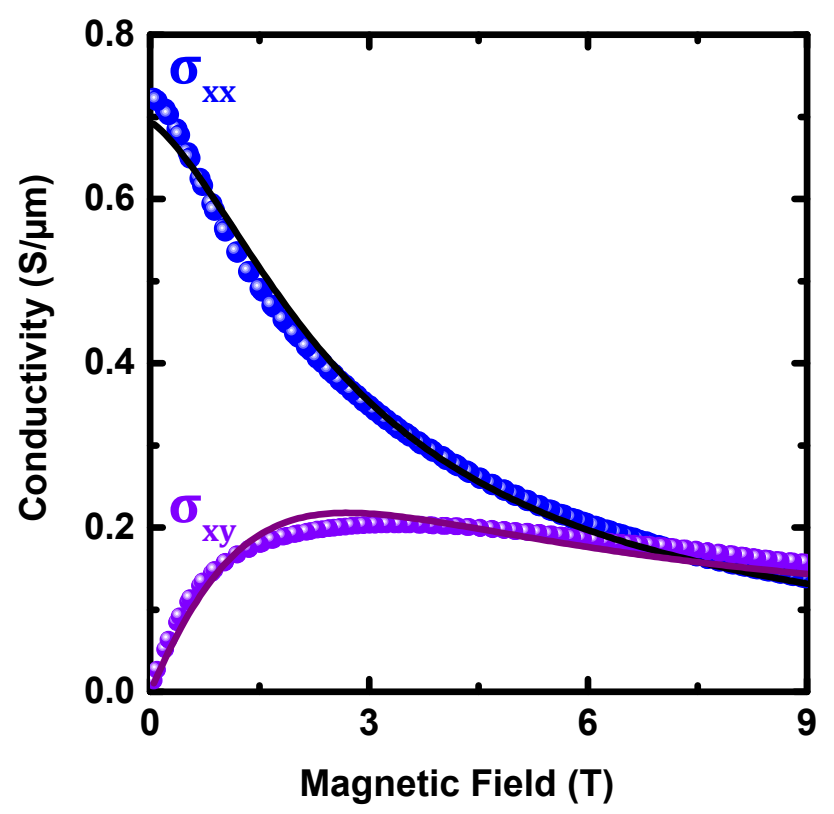

Figure 3.4: Simultaneous fits of longitudinal $\left(\sigma_{x x}\right)$ and transverse resistivity $\left(\sigma_{x y}\right)$ of a $70 \mathrm{~nm} \mathrm{Bi}_{2} \mathrm{Te}_{3}$ film. The $\sigma_{x x}$ (blue curve) and $\sigma_{x y}$ (violet curve) are obtained from the measured longitudinal and transverse resistivities. The magnetoconductance is fitted (solid lines) with the helical magnetoresistance model. With two fitting parameters and a warping parameter $\lambda=90 \mathrm{eV} \AA^{3}$, a decent fit is obtained.

\subsubsection{Linear magnetoresistance in $\mathrm{Bi}_{2} \mathrm{Te}_{3}$ thin films}

Next, we introduce the open question regarding the origin of the observed linear magnetoresistance behaviour in TI materials. In addition to Shubnikov-de Haas oscillations, which are quantum transport phenomena, a strong background magnetoresistance (MR) is often measured in 3D Tls: $\mathrm{Bi}_{2} \mathrm{Te}_{3}[10,16], \mathrm{Bi}_{2} \mathrm{Se}_{3}[17,42], \mathrm{Bi}_{2} \mathrm{Te}_{2} \mathrm{Se}$ [43], Heusler Tls [44, 45] and recently in Dirac semimetals $[46,47]$. For 3D Bi-based Tls, several groups demonstrated a magnetoresistance ranging from a few percent to more than $600 \%[16,48]$. The largest magnetoresistance measured in our $\mathrm{Bi}_{2} \mathrm{Te}_{3}$ thin films is around $150 \%$ as depicted in Fig. 3.3(a). In contrast to the negative magnetoresistive behaviors, such as giant magnetoresistance [49] and colossal magnetoresistance [50], whose mechanisms are well understood, the positive linear magnetoresistance (LMR) in Tls has been extensively reported, but its mechanism is not yet fully understood. The measured LMR in Dirac systems is positive for both field directions, thereby ruling out Hall component admixtures due to misaligned electrodes [48]. A number of mechanisms have been proposed to explain the positive LMR behaviour. Abrikosov proposed a quantum MR model to account for LMR, suggesting that the LMR behaviour arises from a linearly dispersed electron system when all the electrons are filled into the first Landau level (LL) [51, 52]. For all electrons to solely occupy the lowest $\mathrm{LL}$, both the Fermi energy $\left(E_{F}\right)$ and thermal energy $\left(k_{B} T\right)$ have to be much smaller than the energy difference between the lowest and next Landau level; which is known as the extreme quantum limit. Recently, it was proposed that, as long as the system has a positive $g$ factor, the LMR could still appear when multiple LLs are filled, even when thermal smearing is severe [53]. Apart from these descriptions that involve the LLs, also the 
inhomogeneities in disordered conductors can give rise to LMR, as described by the Parish and Littlewood model $[54,55]$. The later model was used to explain the observed LMR in $\mathrm{Ag}_{2 \pm \delta} \mathrm{Te}$ and $\mathrm{Ag}_{2 \pm \delta}$ Se systems, where these materials were modelled as a network of resistors due to disorder-induced mobility fluctuations [54].

The reported LMR in Bi-based and Heusler Tls has been sometimes associated with the Dirac surface states present in these systems $[16,18,56]$. Nevertheless, only a few MR studies on these $\mathrm{TI}$ systems are in the required extreme quantum limit regime. Thus, a full theory explaining the measured LMR behaviour in TI systems is needed.

In Ref. [33], we describe a mechanism that provides $\mathrm{MR}^{6}$, which can be applied to a wide class of systems with spin-orbit coupling (from weak to strong), either at surfaces, interfaces or in the bulk of various systems. Due to spin-momentum locking, states can be orthogonal (for backscattering) or have a reduced overlap (for other angles) upon scattering. The resulting increased conductance is destroyed when the magnetic field aligns the spins, e.g. when the Zeeman energy is larger than the spin-orbit energy [33]. This model applies for example to a topological surface state sufficiently close to the Dirac point, or to the case of Rashba spinorbit coupling. In the latter case, one would have to argue why the Rashba spin-orbit coupling survives away from the surface over the thickness of our thin film and why scattering between the two Rasbha states is suppressed. Instead, it has been shown recently [57] that the spinors of the $\mathrm{TI}$ bulk conduction band states also show a similar effect and that interband scattering is suppressed, warranting the use of this MR model. To describe the measured LMR behaviour in our $\mathrm{Bi}_{2} \mathrm{Te}_{3}$ thin films, we use this MR model [33]. In Fig. 3.4, we fit the MR model to measurement on a $70 \mathrm{~nm}$ thin film of $\mathrm{Bi}_{2} \mathrm{Te}_{3}$. Most importantly, we can fit the longitudinal and transverse conductivity data obtained from the measured longitudinal and transverse resistance simultaneously. In the case when the quadratic mass term dominates over the Zeeman and spinorbit energy, the Fermi energy is given by $E_{F}=\beta k_{F}^{2}$ where $k_{F}$ is extracted from the Hall carrier density $\left(1 / e R_{H}=5.8 \times 10^{13} \mathrm{~cm}^{-2}\right)$. Taking a literature value for the in-plane band mass of $\mathrm{Bi}_{2} \mathrm{Te}_{3}$ conduction band $\left(\beta=45 \mathrm{eV} \AA^{2}\right)[58,59]$, the Fermi energy is determined from the Hall carrier density and calculated to be $32 \mathrm{meV}$. The best fit is found for a zero-field mobility of $\mu=3250 \mathrm{~cm}^{2} \mathrm{~V}^{-1} \mathrm{~s}^{-1}$ and a Zeeman-to-spin-orbit ratio $\frac{g \mu_{B}}{\alpha k_{F}}=0.11 \mathrm{~T}^{-1}$. For a $g$-factor of 12 (which could be sample specific, judging from the range of literature values [60]), the spin-orbit parameter would be $\alpha=0.21 \mathrm{eVA}$, from which the spin-orbit energy at the Fermi energy can be extracted $E_{S O}=\alpha k_{F}$, which is higher than for the $\mathrm{Bi}_{2} \mathrm{Te}_{3}$ thicker flakes [33].

\footnotetext{
${ }^{6}$ The MR model was developed by D. P. Leusink and A. Brinkman.

The author of this $\mathrm{PhD}$ dissertation contributed to this manuscript by growing, performing electronic transport measurements and data analysis of thin film sample presented in the manuscript.
} 


\subsection{Conclusion and outlook}

In summary, we have investigated magnetotransport in $\mathrm{Bi}_{2} \mathrm{Te}_{3}$ thin films. Our magnetoresistance data demonstrate topological nature of the transport, such as a non-trivial Berry phase in $\mathrm{Bi}_{2} \mathrm{Te}_{3}$ samples. The measured nonlinearity in the Hall resistivity reveals the presence of multiple bands or scattering amplitude (mobility) that depends on field. The two-band analysis of the measured $R_{x y}$ did not correctly reproduce the longitudinal resistance behaviour. Using the recently developed MR model, we are able to fit the longitudinal and transverse conductivity data simultaneously. We measured a linear magnetoresistance of about $150 \%$ in $\mathrm{Bi}_{2} \mathrm{Te}_{3}$ thin films. The large and linear magnetoresistance behaviour in Tls has been proposed to be associated with the fact that Tls have Dirac surface states. However, there is still an ongoing debate about whether the reported large LMR don't actually originate from a spin-orbit splitting in the bulk or from the trivial nontopological surface states in TI samples [33]. The mobility $\mu_{\text {Hall }}$ extracted from Hall data is closer to $\mu_{S d H}$ estimated from $\mathrm{SdH}$ oscillations. This agreement is most likely an indication that bulk states contribute to the measured $\mathrm{SdH}$ oscillations. A promising route to reach high quality $\mathrm{TI}$ thin films with topological surface states and without a contribution from the bulk bands at the Fermi energy is to prepare the desired samples and carry out all the characterizations under ultrahigh vacuum conditions without exposing samples to ambient conditions. In the next chapter 4 , we will demonstrate that we can prepare $\mathrm{Bi}_{2} \mathrm{Te}_{3} \mathrm{TI}$ thin films with the Fermi level in the bulk band gap without counterdoping. 


\section{Bibliography}

[1] Z. Alpichshev, J. G. Analytis, J. -H. Chu, I. R. Fisher, Y. L. Chen, Z. X. Shen, A. Fang and A. Kapitulnik, Phys. Rev. Lett. 104, 016401 (2010).

[2] S. E. Harrison, S. Li, Y. Huo, B. Zhou, Y. L. Chen and J. S. Harris, Appl. Phys. Lett. 102, 171906 (2013).

[3] Thomas Ihn, Semiconductor nanostructures: quantum states and electronic transport, Oxford University Press, $1^{\text {st }}$ Edition (2010).

[4] Datta Supriyo, Electronic transport in mesoscopic systems, Cambridge University Press , $9^{\text {th }}$ printing (2012).

[5] Roland Winkler, Spin-orbit coupling effects in two-dimensional electron and hole systems, STMP 191, Springer-Verlag, Berlin (2003).

[6] H. Steinberg, J.B. Laloë, V. Fatemi, J. S. Moodera and P. Jarillo-Herrero, Phys. Rev. B 84, 233101 (2011).

[7] A. A. Taskin, S. Sasaki, K. Segawa and Y. Ando. Phys. Rev. Lett. 109, 066803 (2012).

[8] Y. S. Kim, M. Brahlek, N. Bansal, E. Edrey, G. A. Kapilevich, K. lida, M. Tanimura, Y. Horibe, S.W. Cheong and S. Oh, Phys. Rev. B 84, 073109 (2011).

[9] H. T. He, G. Wang, T. Zhang, I. K. Sou, G. K. L. Wong, J. N. Wang, H. Z. Lu, S. Q. Shen and F. C. Zhang, Phys. Rev. Lett. 106, 166805 (2011).

[10] J. Xiong, Y. Luo, Y. Khoo, S. Jia, R. J. Cava and N. P. Ong, Phys. Rev. B 86, 045314 (2012).

[11] Z. Ren, A. A. Taskin, S. Sasaki, K. Segawa and Y. Ando. Physical Rev. B, 82241306 (2010). 
[12] M. Veldhorst, M. Snelder, M. Hoek, T. Gang, V. K. Guduru, X. L. Wang, U. Zeitler, W. G. van der Wiel, A. A. Golubov, H. Hilgenkamp and A. Brinkman, Nature Mater. 11, 417 (2012).

[13] X. Yu, L. He, M. Lang, W. Jiang, F. Xiu, Z. Liao, Y. Wang, X. Kou, P. Zhang, J. Tang, G. Huang, J. Zou and K. L. Wang, Nanotechnology 24, 015705 (2013).

[14] C. Brüne, C. X. Liu, E. G. Novik, E. M. Hankiewicz, H. Buhmann, Y. L. Chen, X. L. Qi, Z. X. Shen, S. C. Zhang and L.W. Molenkamp, Phys. Rev. Lett. 106, 126803 (2011).

[15] D. -X. Qu, Y. S. Hor, J. Xiong, R. J. Cava and N. P. Ong, Science 329, 821 (2010).

[16] X. Wang, Y. Du, S. Dou and C. Zhang, Phys. Rev. Lett. 108, 266806 (2012).

[17] G. Zhang, H. Qin, J. Chen, X. He, L. Lu, Y. Li and K. Wu, Adv. Funct. Mater. 21, 2351 (2011).

[18] H. Tang, D. Liang, R. L. J. Qiu and X. P. A. Gao, ACS Nano 5, 7510 (2011).

[19] H. Peng, K. Lai, D. Kong, S. Meister, Y. Chen, X.L. Qi, S. C. Zhang, Z.X. Shen and Y. Cui. Nature Mater. 9, 225 (2010).

[20] M. Safdar, Q. Wang, M. Mirza, Z. Wang, K. Xu and J. He. Nano Lett. 13, 5344 (2011).

[21] A. Kandala, A. Richardella, D. Zhang, T. C. Flanagan and N. Samarth. Nano Lett. 13, 2471 (2013).

[22] S. Hikami, A.I. Larkin and Y. Nagaoka, Prog. Theor. Phys. 63, 707 (1980).

[23] G. Bergmann, Phys. Rep. 107, 1 (1984).

[24] T. Hassenkam, S. Pedersen, K. Baklanov, A. Kristensen, C.B. Sorensen, P.E. Lindelof, F.G. Pikus and G.E. Pikus. Phys. Rev. B 55, 9298 (1997).

[25] A. Prinz, G. Brunthaler, Y. Ueta, G. Springholz, G. Bauer, G. Grabecki, T. Dietl. Phys. Rev. B 20, 12983 (1999).

[26] See in the Supplementary Information of: L. Wu, R. Valdés Aguilar, A.V. Stier, C.M. Morris, Y. Lubashevsky, L.S. Bilbro, N. Bansal, S. Oh, N.P. Armitage, Nature Phys. 9, 410 (2013).

[27] H.-Z. Lu and S.-Q. Shen, Phys. Rev. Lett. 112, 146601 (2014).

[28] S. -P. Chiu and J. -J. Lin, Phys. Rev. B 87, 035122 (2013). 
[29] J. Wang, A. M. DaSilva, C. -Z. Chang, K. He, J. K. Jain, N. Samarth, X. -C. Ma, Q. -K. Xue and M. H. W. Chan, Phys. Rev. B 83, 245438 (2011).

[30] N. Bansal, Y. S. Kim, M. Brahlek, E. Edrey and S. Oh, Phys. Rev. Lett. 109, 116804 (2012).

[31] H. Steinberg, D. R. Gardner, Y-S. Lee, P. Jarillo-Herrero, Nano Lett. 10, 5032 (2010).

[32] Y. Ando, J. Phys. Soc. Jpn. 82, 102001 (2013).

[33] D.P. Leusink, R.G.J. Smits, P. Ngabonziza, X. L. Wang, S. Wiedmann, U. Zeitler and A. Brinkman, http://arxiv.org/abs/1412.4065 (2014).

[34] N. W. Ashcroft and N. D. Mermin, Solid State Physics, $1^{\text {st }}$ Edition. Rinehart and Winston, New York, (1976).

[35] D. Shoenberg, Magnetic Oscillations in Metals, Cambridge University Press, Cambridge, (1984).

[36] E. Lahoud, E. Maniv, M. Shaviv Petrushevsky, M. Naamneh, A. Ribak, S. Wiedmann, L. Petaccia, Z. Salman, K. B. Chashka, Y. Dagan and A. Kanigel, Phys. Rev. B 88, 195107 (2013).

[37] L. H. Bao, L. He, N. Meyer, X. F. Kou, P. Zhang, Z. G. Chen, A.V. Fedorov, J. Zou, T. M. Riedemann, T. A. Lograsso,K. L.Wang, G. Tuttle and F. X. Xiu, Sci. Rep. 2, 726 (2012).

[38] H. Cao, J. Tian, I. Miotkowski, T. Shen, J. Hu, S. Qiao and Y. P. Chen, Phys. Rev. Lett. 108, 216803 (2012).

[39] M. Bianchi, D. Guan, S. Bao, J. Mi, B. B. Iversen, P. D. C. King and P. Hofmann, Nature Commun. 1, 128 (2010).

[40] L. D. Landau and E. M. Lifshitz. Course of theoretical physics., $1^{\text {st }}$ Edition, Robert Maxwell, M. C., Oxford-UK, (1981).

[41] J. G. Analytis, R. D. McDonald, S. C. Riggs, J.-H. Chu, G. S. Boebinger and I. R. Fisher, Nat. Phys. 6, 960 (2010).

[42] B. F. Gao, P. Gehring, M. Burghard and K. Kern, Appl. Phys. Lett. 100, 212402 (2012).

[43] B. A. Assaf, T. Cardinal, P. Wei, F. Katmis, J. S. Moodera and D. Heiman, Appl. Phys. Lett. 102, 012102 (2013). 
[44] W. Wang, Y. Du, G. Xu, X. Zhang, E. Liu, Z. Liu, Y. Shi, J. Chen, G. Wu, X. Zhang, Sci. Rep. 3, 2181 (2013).

[45] Z. Hou, Y. Wang, E. Liu, H. Zhang, W. Wang and G. Wu, Appl. Phys. Lett. 107, 202103 (2015).

[46] M. Novak, S. Sasaki, K. Segawa and Y. Ando, Phys. Rev. B 91, 041203(R) (2015).

[47] J. Feng, Y. Pang, D. Wu, Z. Wang, H. Weng, J. Li, X. Dai, Z. Fang, Y. Shi and L. Lu, Phys. Rev. B 92, 081306(R) (2015).

[48] M. Veldhorst, M. Snelder, M. Hoek, C. G. Molenaar, D. P. Leusink, A. A. Golubov, H. Hilgenkamp and A. Brinkman, Phys. Status Solidi RRL 7, 1 (2013), and the references therein.

[49] M. N. Baibich, J. M. Broto, A. Fert, F. Nguyen Van Dau, F. Petroff, P. Etienne, G. Creuzet, A. Friederich and J. Chazelas, Phys. Rev. Lett. 61, 2472 (1988).

[50] A. P. Ramirez, J. Phys.: Condens. Matter 9, 8171 (1997), and the references therein.

[51] A. A. Abrikosov, Phys. Rev. B 58, 2788 (1998).

[52] A. A. Abrikosov, Phys. Rev. B 61, 7770 (2000).

[53] C. M. Wang and X. L. Lei, Phys. Rev. B 86, 035442 (2012).

[54] M. M. Parish and P. B. Littlewood, Nature (London) 426, 162 (2003).

[55] M. M. Parish and P. B. Littlewood, Phys. Rev. B 72, 094417 (2005).

[56] H. He, B. Li, H. Liu, X. Guo, Z. Wang, M. Xie, J. Wang, Appl. Phys. Lett. 100, 032105 (2012).

[57] A. Brinkman, private communication, publication in preparation (2016).

[58] C. Liu, X. Qi, H. Zhang, X. Dai, Z. Fang, S. Zhang, Phys. Rev. B 82, 045122 (2010).

[59] P. Sengupta, T. Kubis, Y. Tan, G. Klimeck, J. Appl. Phys. 117, 044304 (2015).

[60] P. Drath, G. Landwehr, Phys. Lett. A 24, 504 (1967); H. Köler, Phys. Stat. Sol. (b) 75, 127 (1976); C. W. Rischau, B. Leridon, B. Fauque, V. Metayer, C. J. van der Beek, Phys. Rev. B 88, 205207 (2013).

[61] T. Yoshida, S. Fujimoto and N. Kawakami, Phys. Rev. B 85, 125113 (2012). 


\section{4 \\ In-situ spectroscopy of intrinsic $\mathrm{Bi}_{2} \mathrm{Te}_{3}$ thin films and impact of extrinsic defects}

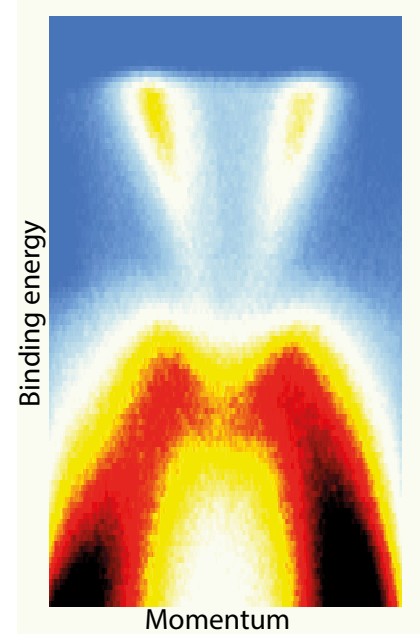

Combined in-situ $x$-ray photoemission spectroscopy, scanning tunnelling spectroscopy and angle resolved photoemission spectroscopy of molecular beam epitaxy grown $\mathrm{Bi}_{2} \mathrm{Te}_{3}$ on lattice mismatched substrates reveal high quality stoichiometric thin films with topological surface states without a contribution from the bulk bands at the Fermi energy. The absence of bulk states at the Fermi energy is achieved without counter doping. We observe that the surface morphology and electronic band structure of $\mathrm{Bi}_{2} \mathrm{Te}_{3}$ are not affected by in-vacuo storage and exposure to oxygen, whereas major changes are observed when exposed to ambient conditions.

This chapter was published as: P. Ngabonziza, R. Heimbuch, N. de Jong, R. A. Klaassen, M. P. Stehno, M. Snelder, A. Solmaz, S. V. Ramankutty, E. Frantzeskakis, E. van Heumen, G. Koster, M. S. Golden, H. J. W. Zandvliet, and A. Brinkman, Physical Review B 92, 035405 (2015). 


\subsection{Introduction}

Topological insulators (TIs) are materials with an insulating bulk interior and spin-momentumlocked metallic surface states as a result of a band inversion from large spin-orbit interaction [1, 2]. Bismuth telluride $\left(\mathrm{Bi}_{2} \mathrm{Te}_{3}\right)$ is one of the $3 \mathrm{D} \mathrm{TI}$ materials that has received a considerable amount of attention as a potential candidate for room temperature spintronics and quantum computational devices [3]. However, despite significant progress in bulk preparation techniques of $\mathrm{TI}$ materials, growing high-quality bulk $\mathrm{Bi}_{2} \mathrm{Te}_{3}$ crystals with a low number of defects and without shunt conduction through the bulk of the material is still a major challenge. The bulk carrier conduction complicates the direct exploitation of the remarkable properties of TI surfaces.

Molecular beam epitaxy (MBE) is an established method for growing high-quality crystalline thin films of Tls with surface-dominated conduction $[4,5,6]$. Using this technique, it has been possible to prepare thin films that are insulating in the bulk. By varying the growth parameters and using substrates with negligible lattice mismatch, bulk insulating thin films of $\mathrm{Bi}_{2} \mathrm{Te}_{3}$ have been synthesized $[7,8,9]$. It would be good, on the other hand, to investigate the growth mode of high quality intrinsic $\mathrm{Bi}_{2} \mathrm{Te}_{3}$ films on lattice mismatched substrates and especially on insulating substrates (with high relative dielectric constant), as these offer the prospect of strong, gate-induced modulation of the sample's carrier density [10].

One of the current challenges, though, for realizing $\mathrm{TI}$ devices, is that grown films are often exposed to air during mounting of contacts or subsequent device fabrication steps. This leads to possible shifts of the Fermi level which result in enhanced bulk conductance $[11,12]$. To date, it is not well-understood how ex-situ contamination processes affect surface and bulk states of $\mathrm{Bi}_{2} \mathrm{Te}_{3}$ films so that precautions can be taken before taking them ex-situ for further investigations. Recently, it was shown for bulk-insulating $\mathrm{Bi}_{2} \mathrm{Te}_{3}$ films that pure oxygen exposure, at low pressure $\left(10^{-6}\right.$ mbar) has no significant influence on their charge transport properties [9]. Nevertheless, since most magnetotransport studies and fabrication processes are carried out in ambient conditions, it is good to understand the impact of oxygen at atmospheric pressure, exposure to air and other ex-situ contaminations. For these investigations, it is necessary to have high quality films since their inertness to oxidation, for example, depends on the amount of surface defects and grain boundaries.

In this work, we first focus on optimizing our growth procedure in order to realize bulk insulating films grown on lattice mismatched [13] insulating substrates $\left(\mathrm{Al}_{2} \mathrm{O}_{3}[0001]\right.$ and $\left.\mathrm{SrTiO}_{3}[111]\right)$. Secondly, we perform a systematic in-situ characterization to investigate the effect of aging/degradation due to any vacancies or antisite defects for films stored in-vacuo. Thirdly, our in-situ angle resolved 
photoemission spectroscopy (ARPES) experiments clearly reveal topological Dirac surface states, consistent with a linear increase in the density of states (DOS) measured by in-situ scanning tunneling spectroscopy. Lastly, we study the effect of pure oxygen exposure at atmospheric pressure; and then the effect of ex-situ contamination in air. We use x-ray photoemission spectroscopy (XPS) for the characterization and detection of any $\mathrm{Te} / \mathrm{Bi}$ excess in our films, and we employ low temperature scanning tunnelling spectroscopy (STS) to acquire differential conductivity spectra for samples kept in-situ, then exposed to pure oxygen at atmospheric pressure and later to air. Our STS analysis is further confirmed by in-situ ARPES investigations at low temperature and room temperature for samples kept in-situ and later exposed to ambient conditions.

Our spectroscopy data (STS and ARPES) reveal that the as-grown $\mathrm{Bi}_{2} \mathrm{Te}_{3}$ films on lattice mismatched insulating substrates possess an intrinsically topological electronic structure, meaning that the Fermi level only crosses the topologically non-trivial metallic surface states. This is achieved without any counterdoping. Combining in-situ and ex-situ spectroscopy and topography data, we find that in-situ storage in ultra-high vacuum and (short) exposure to pure oxygen at atmospheric pressures leave the band structure at the surface unaffected whereas breaking vacuum and exposing the sample surface to air results in notable changes in the surface band structure spectra and topography of the films.

\subsection{Films growth and characterization}

Thin films of $\mathrm{Bi}_{2} \mathrm{Te}_{3}$ were grown on c-plane sapphire $\mathrm{Al}_{2} \mathrm{O}_{3}[0001]$ and $\mathrm{SrTiO}_{3}[111]$ (STO) substrates using MBE by co-evaporating high purity $\mathrm{Te}(99.999 \%)$ and $\mathrm{Bi}(99.999 \%)$ in a $\mathrm{Te}$ rich environment. The base pressure in the deposition chamber was lower than $5 \times 10^{-10} \mathrm{mbar}$. During deposition, the flux ratio Te/Bi was kept at about 10 , the growth rate at $\sim 4 \AA / \mathrm{min}$ and the highest pressure recorded was $2.8 \times 10^{-8}$ mbar. Before introducing the sapphire substrates in the MBE chamber, they were cleaned with acetone and ethanol in an ultrasonic bath, then annealed at $1050^{\circ} \mathrm{C}$ for 1 hour at atmospheric conditions. This cleaning procedure resulted in straight step edges and atomically flat terraces at the substrate surface, with a width of $\sim 150$ $\mathrm{nm}$. For the STO [111] substrates, to achieve atomically flat surfaces before loading them into the MBE chamber, we used a method similar to that reported in Refs. [14, 15]. In order to avoid disordered interfacial layers at the interfaces between our films and the substrate, we used a twostep temperature growth scheme $[16,17]$, which results in atomically sharp interfaces between the $\mathrm{TI}$ film and the substrate. The first nucleation layer was deposited at a temperature of $190^{\circ} \mathrm{C}$, then slowly annealed to a higher growth temperature of $230^{\circ} \mathrm{C}$ in order to improve the crystalline 
(a)

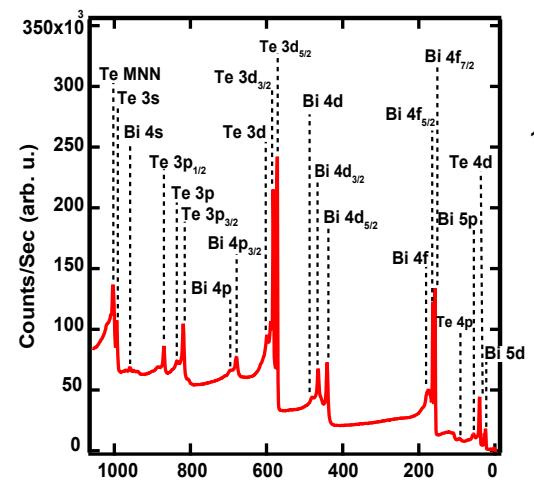

(b)

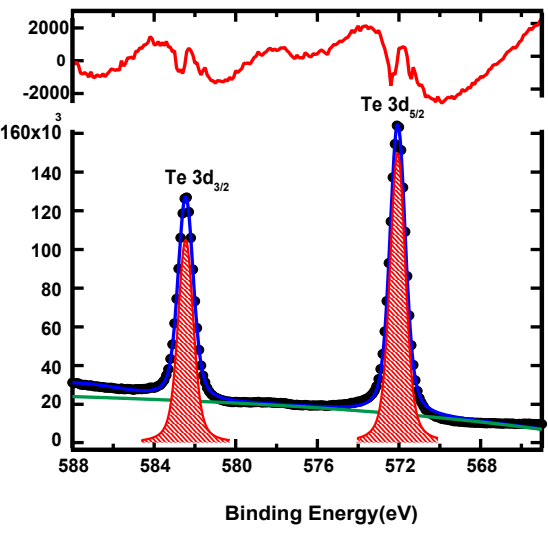

(c)

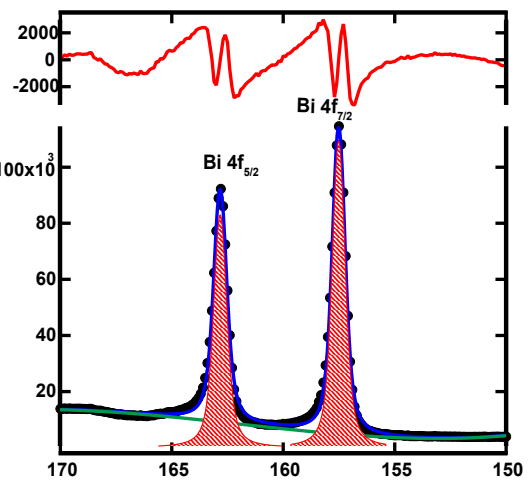

Figure 4.1: In-situ measurements of a $30 \mathrm{~nm} \mathrm{Bi}_{2} \mathrm{Te}_{3}$ film grown on $\mathrm{Al}_{2} \mathrm{O}_{3}[0001]$. (a) General scan. Only $\mathrm{Bi}$ and Te peaks are resolved. (b) and (c) High-resolution scans around the $\mathrm{Te} 3 d$ and $\mathrm{Bi} 4 f$ main peaks, respectively. The red shaded zone shows the areas of the fitted peaks with the background (green line) removed. The upper red curve is the residual after a Shirley background subtraction.

quality. The annealed layer was then used as a template for subsequent epitaxial growth of the second layer. Compared to films grown in a one-step temperature scheme, we observe a much better quality for the two-step growth procedure in terms of a flat morphology, and considerably reduced number of 3D growth defects. Similar significant reduction of 3D structure density has also been reported recently on two-step grown $\mathrm{Bi}_{2} \mathrm{Te}_{3}$ films [17]. To further improve the surface smoothness and quality of the films, we continued to anneal at $230^{\circ} \mathrm{C}$ for an additional 30 minutes; then cooled down to room-temperature at a rate of $3^{\circ} / \mathrm{min}$. This growth procedure yields high quality $\mathrm{Bi}_{2} \mathrm{Te}_{3}$ films. Using this growth procedure, we present in this study spectroscopy data of two samples of 30 and $20 \mathrm{~nm}$ grown on $\mathrm{Al}_{2} \mathrm{O}_{3}$ [0001] and one $15 \mathrm{~nm}$ film grown on $\mathrm{SrTiO}_{3}$ [111]. After growth, samples were characterized in-situ using XPS to investigate the surface elemental composition and stoichiometry. Samples were transferred to XPS without breaking ultra-high vacuum (UHV) conditions since the XPS system is connected to the growth chamber via a distribution chamber. The UHV conditions of this system ensure that films are free from ex-situ contamination. Figure 4.1(a) shows an in-situ XPS scan, where only Bi and Te peaks are resolved with no appearance of extra peaks such as carbon and oxygen as often seen in films exposed to atmosphere $[18,19,20,21]$ (see Fig. 4.10(a) in section 4.5). This lack of other core level signatures is an indication of a clean surface, free from contaminations. The surface chemical stoichiometry was studied by fitting the area under the Te $3 d_{5 / 2}$, Te $3 d_{3 / 2}$ and $\mathrm{Bi} 4 f_{7 / 2}$, $\mathrm{Bi} 4 f_{5 / 2}$ peaks. The ratio is determined to be $1.49 \pm 0.05$. Figures 4.1 (b) and 4.1 (c) show these $\mathrm{Te}$ and $\mathrm{Bi}$ peaks, respectively, together with the Voigt function fits (after subtraction of Shirley 
(a)

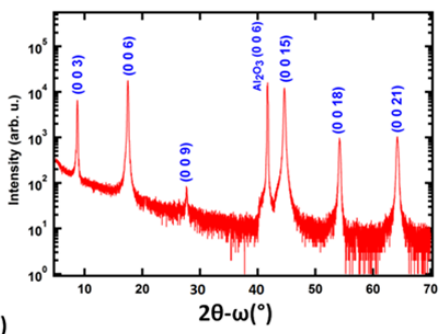

(b)

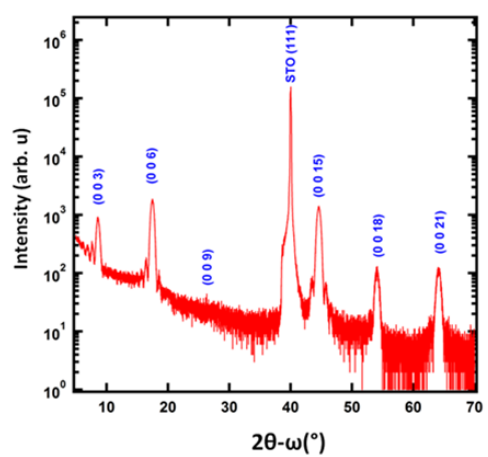

(c)

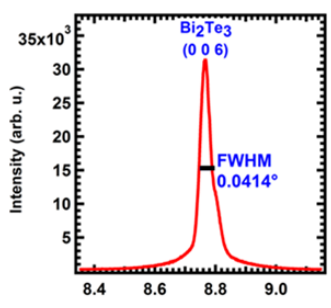

(d)

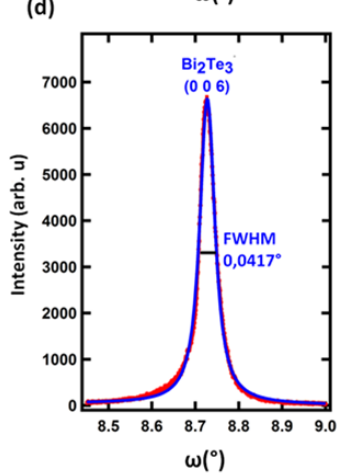

(e)

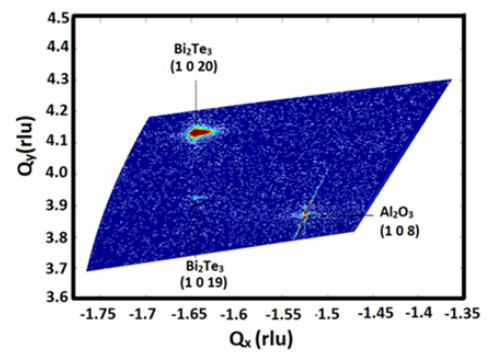

(f)

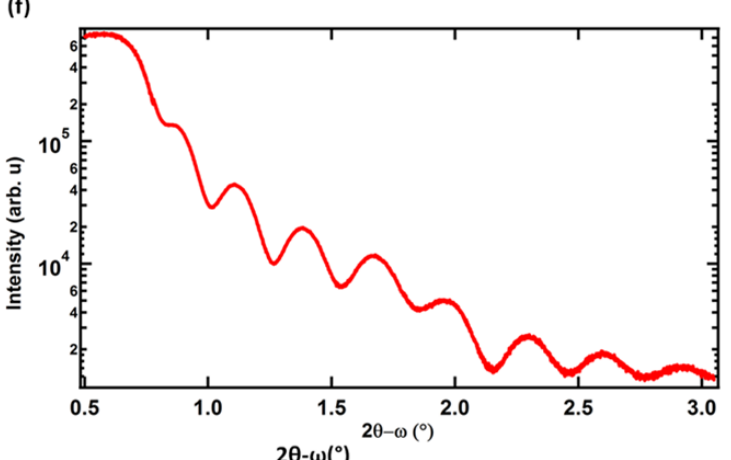

Figure 4.2: Ex-situ XRD measurements. (a) and (b): $2 \theta-\omega$ scans of $\mathrm{Bi}_{2} \mathrm{Te}_{3}$ films grown on sapphire and STO. Only the substrate peaks and the $\left(\begin{array}{lll}0 & 0 & 3\end{array}\right)$ family of $\mathrm{Bi}_{2} \mathrm{Te}_{3}$ diffraction peaks are resolved. (c) and (d): Rocking curves of the $\mathrm{Bi}_{2} \mathrm{Te}_{3}$ main peak at ( 006 ). (e) A 2D reciprocal space map of a $30 \mathrm{~nm}$ film grown on sapphire. Positions of the $\mathrm{Al}_{2} \mathrm{O}_{3}\left(\begin{array}{lll}1 & 0 & 8\end{array}\right)$ and $\mathrm{Bi}_{2} \mathrm{Te}_{3}\left(\begin{array}{ll}1 & 0\end{array}\right.$ $20)$ and ( 1019 ) peaks are shown. (f) X-ray reflectivity data of a $15 \mathrm{~nm}$ film grown on STO showing distinct Kiessig fringes over more than five orders of magnitude in intensity. Despite the significant lattice mismatch between the film and substrates [6, 13], high structural-quality films are grown.

background). The peak positions and their relative intensities are consistent with that of $\mathrm{Bi}_{2} \mathrm{Te}_{3}$ samples reported in the literature [18, 22, 23].

To further confirm the high crystalline quality of our $\mathrm{Bi}_{2} \mathrm{Te}_{3}$ films after in-situ measurements (STM/STS and ARPES), we performed ex-situ x-ray diffraction (XRD) measurements, which confirmed that the films were grown with in-plane and out-of-plane lattice constants $a=4.34 \AA$ and $c=30.41 \AA$, respectively. These lattice parameters are consistent with values reported previously for $\mathrm{Bi}_{2} \mathrm{Te}_{3}$ films $[17,24]$. From XRD measurements of films grown on $\mathrm{Al}_{2} \mathrm{O}_{3}[0001]$ and STO[111] substrates (see Fig. 4.2), in the $2 \theta-\omega$ scan only substrate peaks and the $\left(\begin{array}{lll}0 & 0 & 3\end{array}\right)$ of diffraction peaks from the films are resolved; implying the films are aligned along the c-axis. From the rocking curves, the measured full width half maximum of the $\left(\begin{array}{lll}0 & 0 & 6\end{array}\right)$ peak are $0.0414^{\circ}$ (for films grown on sapphire) and $0.0417^{\circ}$ (for films grown on STO), confirming the excellent quality of our films. Despite the lattice mismatch between $\mathrm{Bi}_{2} \mathrm{Te}_{3}$ [001] and these substrates, smooth 
(a)

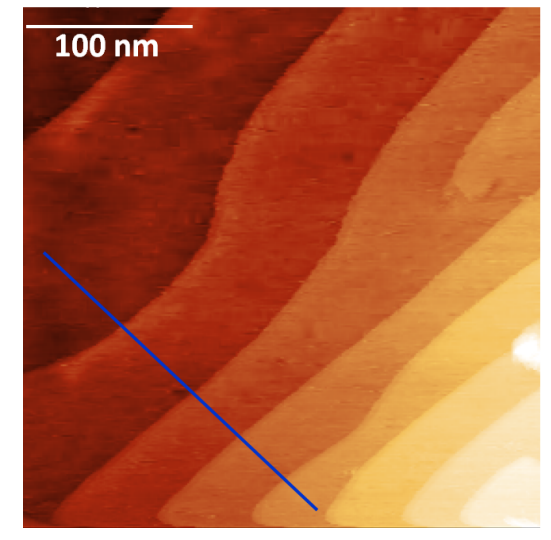

(c) (b)

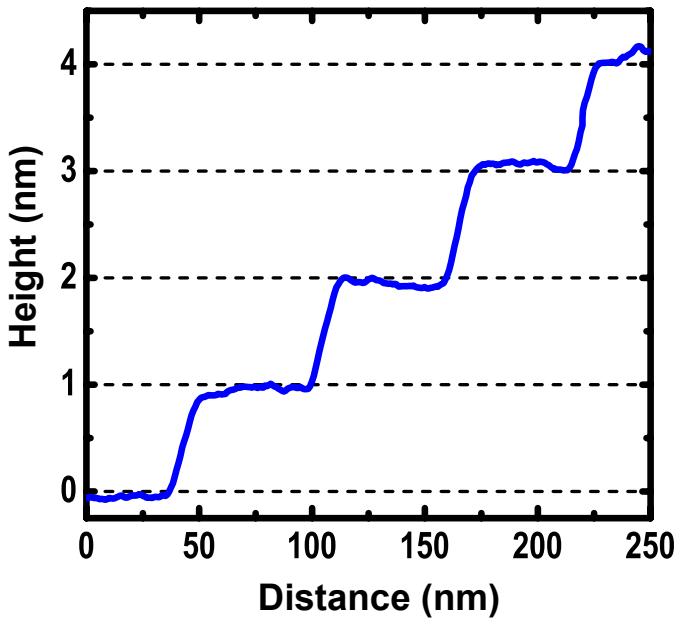

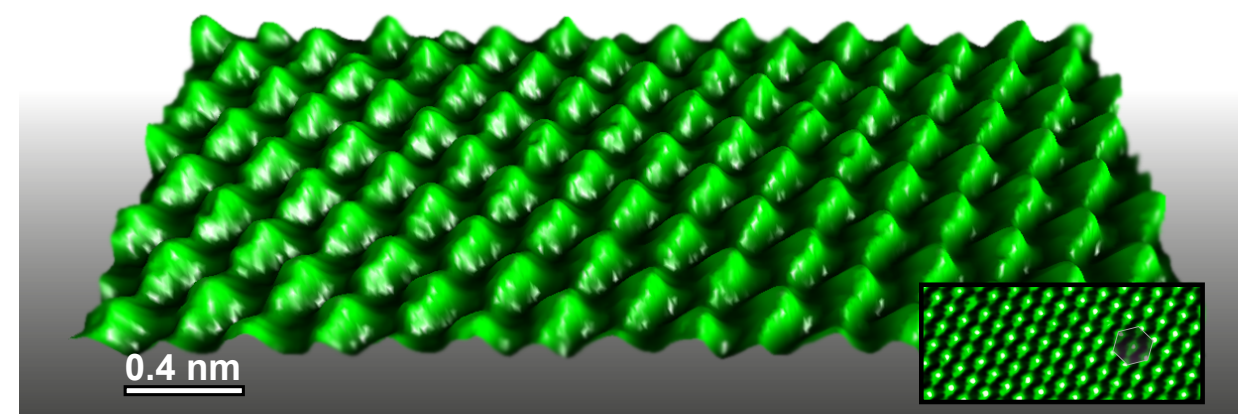

Figure 4.3: Topography of $30 \mathrm{~nm} \mathrm{Bi}_{2} \mathrm{Te}_{3}$ film grown on sapphire. (a) A scan of a $250 \times 250 \mathrm{~nm}^{2}$ area showing smooth film terraces. (b) Line profile across a series of wide steps (blue line). The step height is $\sim 1 \mathrm{~nm}$. (c) Atomic resolution of a $6 \times 2.6 \mathrm{~nm}^{2}$ area showing the surface tellurium atoms with a hexagonal crystal structure (right bottom insert). The secondary lobes are artefacts caused by a slight doubling of the STM tip.

films had formed. This is because of the Van der Waals epitaxy [6, 25, 26], which relaxes the lattice-matching condition required for most common epitaxial growth of covalent semiconductors and their heterostructures.

After in-situ XPS measurements, samples were subsequently transferred to the low temperature STM chamber, which is not connected to the growth chamber. The transfer was done using a UHV suitcase equipped with a nonevaporable getter (NEG) pump [27] together with an ion pump. During the transfer process, the pressure remained lower than $2 \times 10^{-9} \mathrm{mbar}$. Once samples were transferred to the STM, its UHV conditions (in the $10^{-12}$ mbar range) ensured that the film surface remained free from collecting further adsorbates for months. The in-situ STM/STS data were acquired at $77 \mathrm{~K}$. Many topography and spectroscopy maps were taken at random positions 
on the surface of the film at a bias voltage and set-point current of $+350 \mathrm{mV}$ and $1 \mathrm{nA}$. The results were consistent with previously published STM studies on $\mathrm{Bi}_{2} \mathrm{Te}_{3}[28,29,30]$. Figure 4.3(a) shows a typical STM image of the atomically smooth $\mathrm{Bi}_{2} \mathrm{Te}_{3}$ surface. The corresponding height profile across the surface is plotted in Fig. 4.3(b). The step height of adjacent terraces is $10.3 \AA$. This value is consistent with one quintuple layer [2] (QL) of $\mathrm{Bi}_{2} \mathrm{Te}_{3}$ since its stacking sequence along the [001] direction is Te-Bi-Te-Bi-Te, forming a QL height of $1 \mathrm{~nm}$. Fig. 4.3(c) shows an atomic resolution image of our $\mathrm{Bi}_{2} \mathrm{Te}_{3}$ film surface, where the surface tellurium atoms are clearly observed exhibiting a hexagonal unit cell (see right bottom insert of Fig. 4.3(c)). The interatomic spacing of these tellurium atoms was determined to be $4.3 \AA$, corresponding perfectly to the tellurium-terminated surface in $\mathrm{Bi}_{2} \mathrm{Te}_{3}$. No surface adatoms were observed, which is an indication of a clean surface of our high quality thin films.

\subsection{In-situ spectroscopy}

Now, we present spectroscopy data of $\mathrm{Bi}_{2} \mathrm{Te}_{3}$ films. Figure 4.4(a) shows low temperature differential conductivity curves of a $30 \mathrm{~nm}$ film grown on sapphire measured after one day in the STM chamber (blue line), after 6 weeks in-situ storage (green) and later after being oxidized for 10 minutes in $1 \mathrm{~atm}$., pure $\mathrm{O}_{2}$ (red). The spectra were obtained by numerical differentiation of many $\mathrm{I}-\mathrm{V}$ curves [31] with setpoint parameters of $V_{b}=+350 \mathrm{mV}$ at $I=1 \mathrm{nA}$. In this figure, $\mathrm{E}_{\mathrm{B}-\mathrm{BCB}}$ marks the bottom of the bulk conduction band whereas $\mathrm{E}_{\mathrm{T}-\mathrm{BVB}}$, the top of the bulk valence band. $E_{F}$ and $E_{D}$ correspond to the Fermi level and Dirac point, respectively. $E_{B-B C B}$ and $E_{T-B V B}$ are determined by the point where additional states appear with respect to the surface states, and by taking a literature value of $\sim 210 \mathrm{meV}$ for the band gap of $\mathrm{Bi}_{2} \mathrm{Te}_{3}[23,32,33]$. From these spectra, we find linearly increasing DOS, indicative of Dirac surface states residing in the bulk energy gap between the bulk conduction band ( $B C B)$ and bulk valence band (BVB). Since the Dirac point is located in the BVB, by extrapolating this linearly increasing DOS, the Dirac point is approximately at $0.23 \mathrm{eV}$ below $\mathrm{E}_{\mathrm{F}}$. The linear part of the spectra exhibits a plateau appearing at around $-50 \mathrm{mV}$. This latter feature is often attributed to the hexagonal warping of the surface band in $\mathrm{Bi}_{2} \mathrm{Te}_{3}$ samples and has been seen before in STS spectra of $\mathrm{Bi}_{2} \mathrm{Te}_{3}$ thin films and single crystals $[29,30,34]$. A complete description of a cubic warping term in the Hamiltonian for the Dirac cone is given in Ref. [35], the main parameter being $\lambda$, which describes the strength of the hexagonal warping. When fitting the DOS of the warped Dirac cone to the kink feature in the data, we find that the warping alone is not strong enough (for reasonable parameter values) to explain the kink. Including a $k$-dependent tunnelling probability, implying that the states with larger parallel momenta contribute less to the tunnelling current [36], does not significantly 
(a)

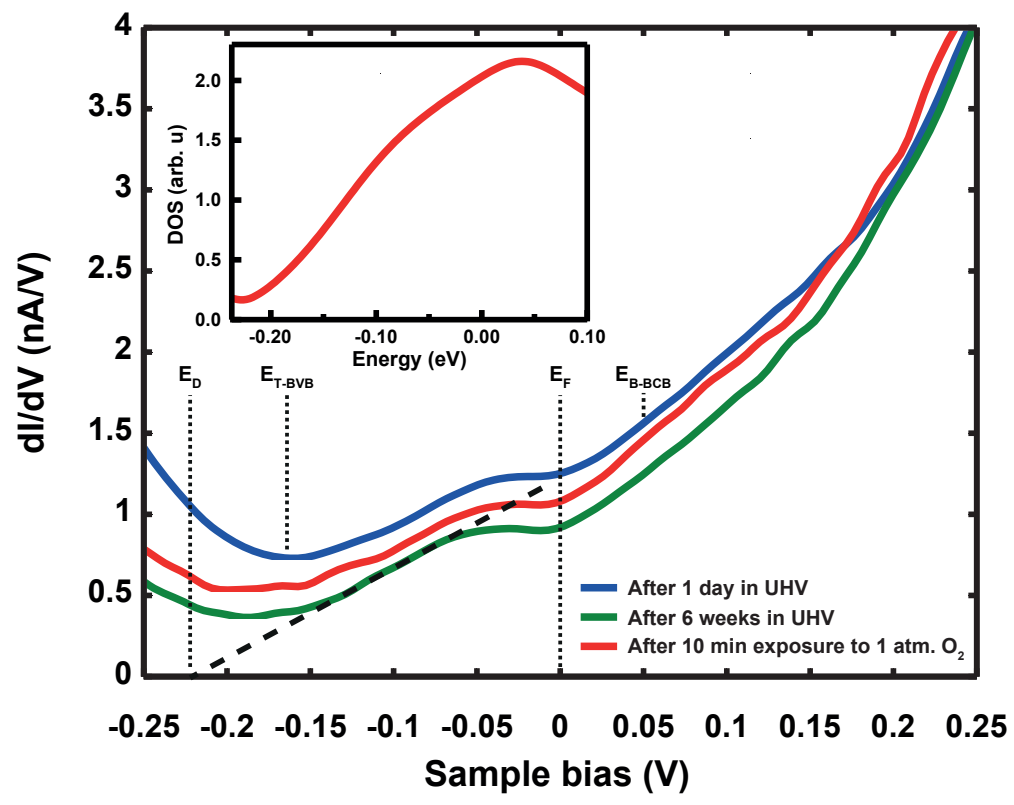

(b)

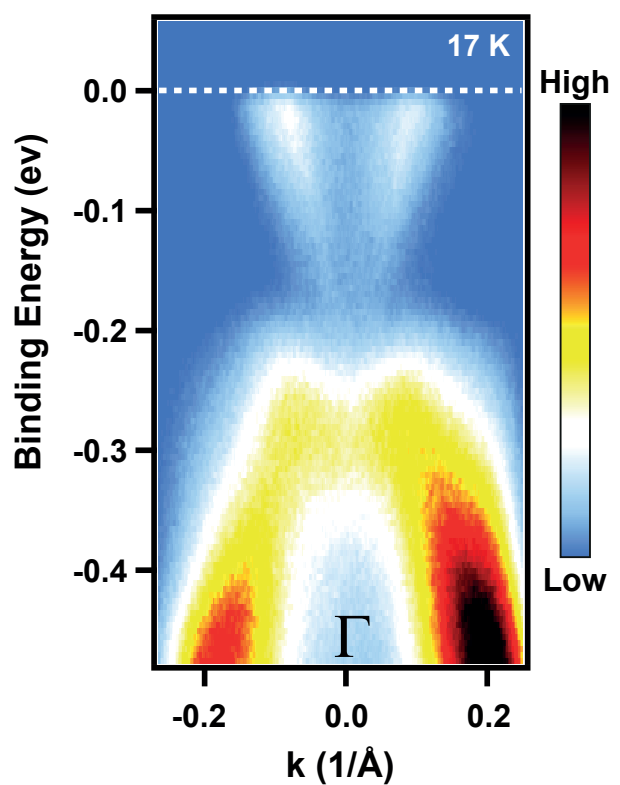

Figure 4.4: ((a) STM spectroscopy measurements at $77 \mathrm{~K}$ on a $30 \mathrm{~nm} \mathrm{Bi}{ }_{2} \mathrm{Te}_{3}$ film (blue curve). Effect of storing films in-situ at $P \sim 10^{-12}$ mbar for weeks (red curve) and exposing them to pure oxygen (green curve). The inset shows calculated density of states as function of energy from the Dirac cone alone. (b) ARPES $I(k, E)$ image around the $\Gamma$ point of a $20 \mathrm{~nm} \mathrm{Bi}_{2} \mathrm{Te}_{3}$ film recorded at $17 \mathrm{~K}$. The white dashed line indicates the position of the Fermi level, which is in the bulk band gap without bulk band contribution. Both samples were grown on sapphire

improve the fit. However, from ARPES measurements along the $\bar{\Gamma}-\bar{M}$ direction, it is known that the Dirac cone bends outwards, as visible also in ab-initio calculations [39]. Tight binding models exist that incorporate this effect [2], and it can also be included in the Dirac Hamiltonian by including a small negative mass term. For reasonable parameter values (see details in section 4.4) that fit the ARPES dispersion curves well, a DOS was calculated as function of energy, shown in the inset of Fig. 4.4(a). These are the DOS from the Dirac cone alone. It is clear that the plateau feature can indeed be qualitatively understood on the basis of the outward bending of the Dirac cone along the $\bar{\Gamma}-\bar{M}$ direction, and that it is not related to the bulk conduction band. These STS data are in agreement with previously published STS data on $\mathrm{Bi}_{2} \mathrm{Te}_{3}$ [29], and indicate that there are no bulk states present at Fermi level.

To further confirm the presence of topological surface states without a contribution from the bulk bands at $\mathrm{E}_{F}$ and also verify larger scale homogeneity of the surface states, we also performed ARPES measurements on $\mathrm{Bi}_{2} \mathrm{Te}_{3}$ films. After growth and XPS characterization, samples were stored in the UHV suitcase for three days and transferred to an ARPES setup. The pressure in 


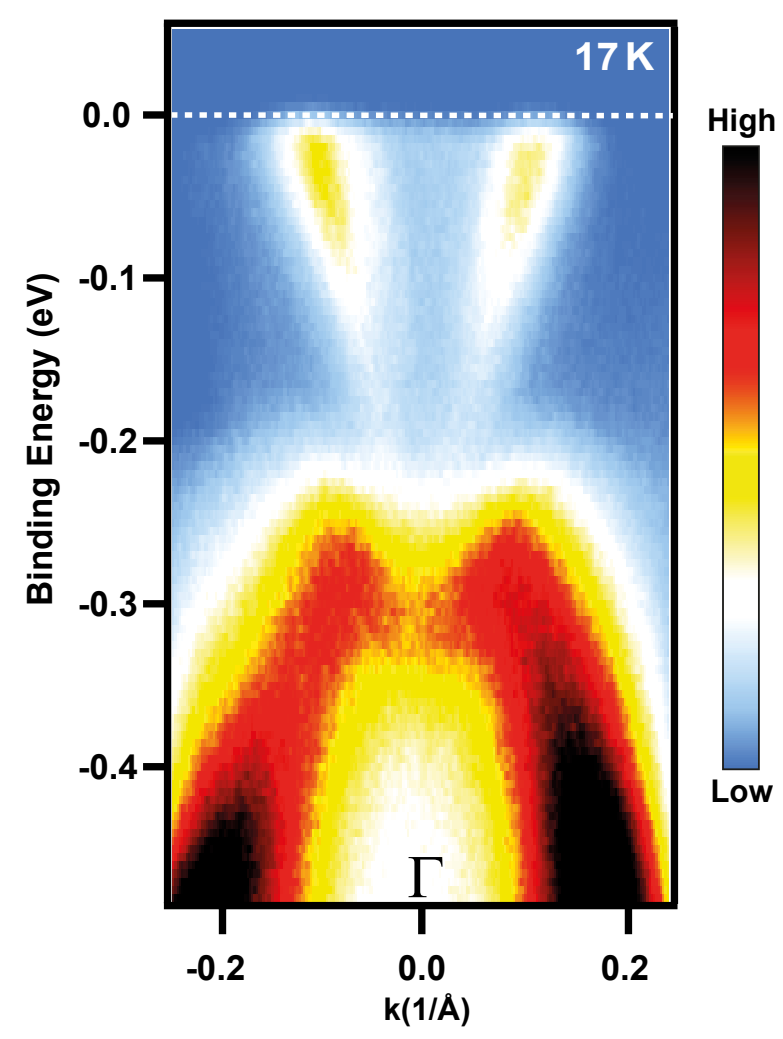

Figure 4.5: ARPES spectra around the $\Gamma$ point of a $15 \mathrm{~nm} \mathrm{Bi} \mathrm{Te}_{3}$ film grown on $\mathrm{SrTiO}_{3}[111]$. As was the case for the film grown on sapphire, for this sample only the topological surface states cross the Fermi level.

the UHV suitcase remained lower than $2 \times 10^{-10}$ mbar throughout this procedure. Fig. 4.4(b) shows a representative ARPES spectrum in the vicinity of the Fermi level of a $20 \mathrm{~nm} \mathrm{Bi}_{2} \mathrm{Te}_{3}$ film grown on sapphire around the $\Gamma$ point. The spectra were taken at a temperature of $\sim 17 \mathrm{~K}$ using a helium photon source of energy $21.2 \mathrm{eV}$. The characteristic ' $\mathrm{V}$ '-shaped surface state is clearly observed, confirming the presence of the topological Dirac cone surface state in our $\mathrm{Bi}_{2} \mathrm{Te}_{3}$ films. The white dashed line shows the position of the Fermi level, which lies well within the bulk band gap with no observable contribution of the bulk conduction band at the Fermi energy. The Fermi velocity is $\hbar v_{F}=2.31 \pm 0.20 \mathrm{eVA}$ which is consistent with previous ARPES studies [17,9] and theoretical calculations [2]. The Dirac point was measured to be at $0.24 \mathrm{eV}$ below $\mathrm{E}_{\mathrm{F}}$, which is in good agreement with the STS measurements for the $30 \mathrm{~nm}$ thick $\mathrm{Bi}_{2} \mathrm{Te}_{3}$ film shown in Fig. 4.4(a) and previously published ARPES data [40]. The fact that there are no bulk bands at $E_{F}$ implies that electrical conduction in these films is only due to the surface states [41], which is desirable for electronic transport experiments.

Here, we emphasize that to stabilize the Fermi level in the band gap with no bulk bands at $E_{F}$, 
no counter-doping was necessary in our MBE-grown thin film samples. Counter-doping reduces the surface mobility and adds disorder in samples $[15,41]$. To show that we have a stable and reproducible procedure for obtaining bulk insulating $\mathrm{Bi}_{2} \mathrm{Te}_{3}$ samples, we have also grown our films on STO[111] substrates. Figure 4.5 depicts an illustrative ARPES $I(k, E)$ image from a $15 \mathrm{~nm}$ $\mathrm{Bi}_{2} \mathrm{Te}_{3}$ film grown on STO. The data from the film grown on STO substrate agree with those from films grown on sapphire in that the Fermi level is clearly situated in the bulk band gap. This finding also confirms that despite large lattice mismatch between the film and the substrate [13], intrinsic $\mathrm{Bi}_{2} \mathrm{Te}_{3}$ films are grown. In particular, one can exploit these bulk insulating $\mathrm{Bi}_{2} \mathrm{Te}_{3}$ films grown on STO for fabrication of hybrid devices consisting of bottom gate-tunable topological insulator interfaced to either a superconductor or ferromagnet, by using STO [111] substrate as a back gate because it has a very high relative dielectric constant at low temperature [10]

To investigate the effect of UHV storage (at room temperature) and oxidation on the band structure of our $\mathrm{Bi}_{2} \mathrm{Te}_{3}$ films, we compare STS spectra of a film measured after one day in the STM chamber, with spectra taken after 6 weeks and after being oxidized for 10 minutes at 1 atm. The oxidation was performed in the load lock of the STM system, at atmospheric pressure. From all three traces in Fig. 4.4(a) it is remarkable to observe that even after keeping films in-situ for weeks and allowing them to interact with pure oxygen, no noticeable degradation and no corresponding shifts in the STS spectra take place. The overall key features: the linear surface band, the plateau due to 'warping', and the position of the Fermi energy relative to the bulk bands, remain unchanged. This observation is further confirmed by the ARPES data shown in Fig. 4.4(b) and Fig. 4.5 since these samples were measured after storage for three days in the UHV suitcase $\left(\mathrm{P} \sim 2 \times 10^{-10} \mathrm{mbar}\right.$ ); and no bulk conduction bands were observed. Aging effects have been reported in ARPES data from bulk single crystals of $\mathrm{Bi}_{2} \mathrm{Te}_{3}$ within hours after cleavage under UHV conditions [32, 42]. To further investigate the effect of UHV exposure on the electronic structure of these films, we performed ARPES measurements over different time intervals in UHV condition, with the results shown in Fig. 4.6 (a)-(d). As for our films, the surface is stable as long as they are kept in-situ in UHV conditions or when exposed to pure oxygen. This conclusion is consistent with recent in-situ four-point probe conductivity, angle resolved photoemission spectroscopy and conductive probe atomic force microscopy studies [9, 43]. 

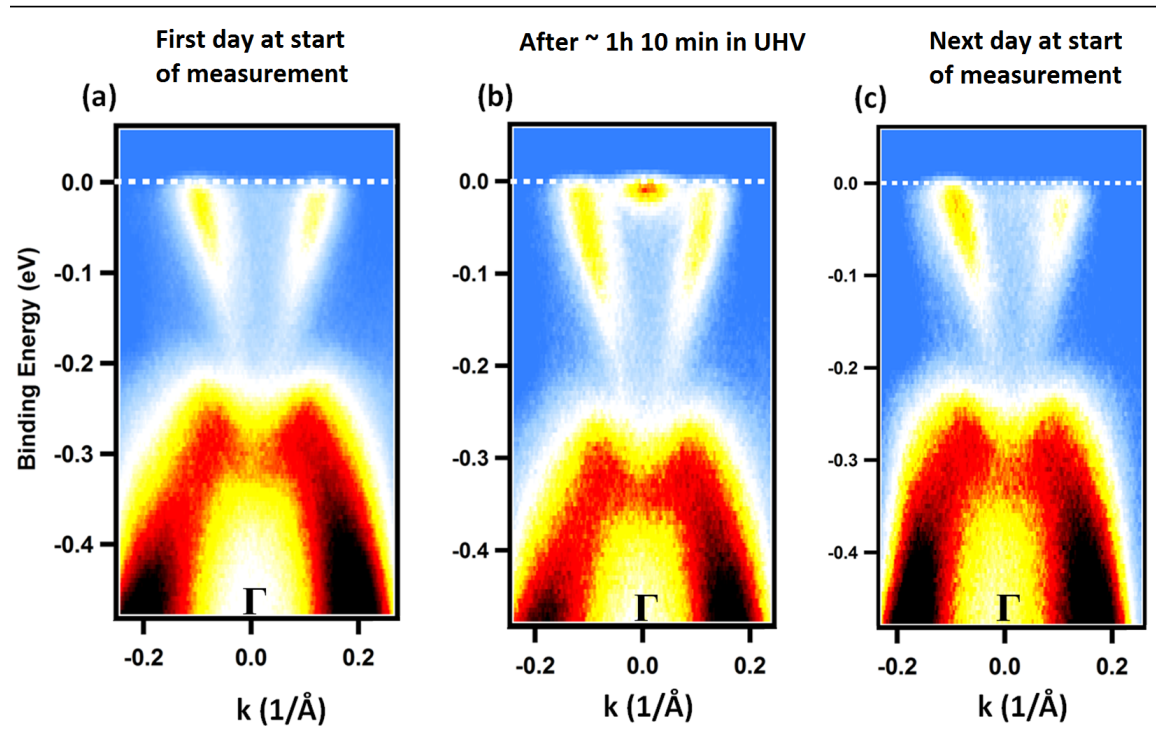

After 55 min in UHV

(d)

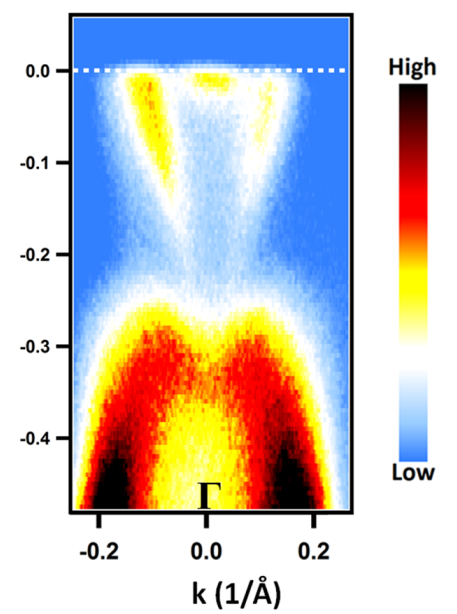

Figure 4.6: ARPES spectra of a $15 \mathrm{~nm}$ thick $\mathrm{Bi}_{2} \mathrm{Te}_{3}$ film grown on $\mathrm{SrTiO}_{3}$ [111]. Measurements were taken over different time intervals in UHV conditions during ARPES measurements. (a) Only topological surface states at the Fermi level are observed at the beginning of the experiment, (b) but after $\sim 1 \mathrm{~h} 10 \mathrm{~min}$ bulk conduction bands start to bend downward due to surface adsorption effect from probably the residual gases $\left(\mathrm{H}_{2}, \mathrm{CO}, \mathrm{N}_{2}\right)$. (c) Raising temperature back to room temperature and allowing the sample to recover for sometime in UHV conditions resets the amount of band bending by desorption of the adatoms. The spectrum was measured after recooling to $\sim 17 \mathrm{~K}$. (d) The downward bulk band bending reappears again after $\sim 55 \min$ during low-temperature ARPES measurements. These observations further support the fact that our films do not degrade in UHV conditions.

\subsection{Model calculation of density of states and dispersion relations}

To model $\mathrm{Bi}_{2} \mathrm{Te}_{3}$ dispersion relations along the $\bar{\Gamma}-\bar{M}$ and $\bar{\Gamma}-\bar{K}$ directions, we used the k.p Hamiltonian described in Ref. [2, 39] to calculate the Green's function and subsequently the Density Of States (DOS). This model includes anisotropic higher order terms, for example describing the outward bending of the Dirac cone along the $\bar{\Gamma}-\bar{M}$ direction at the energy close to the maximum of the valence band, also present in ab-initio calculations [39]. We used as few parameters as possible to describe the measured ARPES dispersions. The parameters used are (in the same notation as Ref. [39]): $m_{1}^{*}=-0.054 \mathrm{eV}^{-1} \dot{A}^{-2}, v=2.55 \mathrm{eV} \dot{A}, \alpha=5.5 \mathrm{eV} \dot{A}^{2}, \lambda=$ $250 \mathrm{eV} \dot{A}^{3}$ and the chemical potential $\mu=225 \mathrm{meV}$ above the Dirac point, which coincides with 

Chapter 4. In-situ spectroscopy of intrinsic $\mathrm{Bi}_{2} \mathrm{Te}_{3}$ thin films and impact of ...
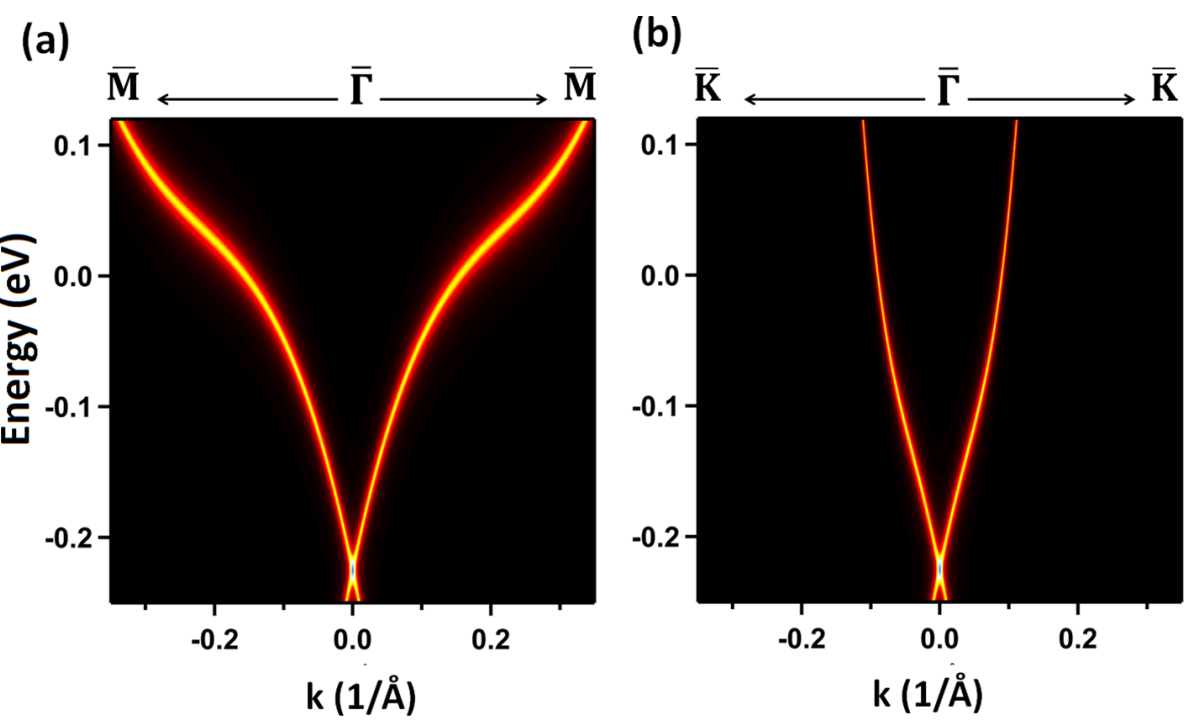

Figure 4.7: Calculated spectral functions of $\mathrm{Bi}_{2} \mathrm{Te}_{3}$ along the (a) $\bar{\Gamma}-\bar{M}$ and (b) $\bar{\Gamma}-\bar{K}$ directions. Outward bending of the Dirac cone is observed only along the $\bar{\Gamma}-\bar{M}$ direction.

the data from the STS experiments. The Green's function was calculated ${ }^{1}$ on a grid of $1200 \times$ $1200 \mathrm{k}$-points with an energy step of $3 \mathrm{meV}$. Figure 4.7(a) and (b) show the spectral functions obtained by summing the imaginary parts of the diagonal components of the Green's function. The DOS, shown as inset in Fig. 4.4(a) in section 4.3, was obtained by integrating the spectral functions over the Brillouin zone.

\subsection{Impact of extrinsic defects}

Next, we study the effect of exposure to ambient conditions. This is relevant for the understanding of how ex-situ contamination processes affect surface and bulk states of $\mathrm{Bi}_{2} \mathrm{Te}_{3}$ films for future ex-situ fabricated hybrid topological insulator devices. For this investigation, we employ both STM/STS and ARPES since they are complementary tools for probing reliably the band structure of materials. Figure 4.8(a) depicts an ARPES $I(k, E)$ image of the $20 \mathrm{~nm} \mathrm{Bi}_{2} \mathrm{Te}_{3}$ film grown on sapphire. This time, the sample was measured at room temperature before exposure to ambient conditions. This $I(k, E)$ image was recorded after the sample had been in the ARPES chamber for 5 days, after measurement of the low- $T$ data shown in Fig. 4.4(b) (which themselves were after three days in UHV suitcase and transfer to ARPES setup). The Fermi level is still lying below the bottom of the bulk conduction band (B-BCB). From this room temperature ARPES

\footnotetext{
${ }^{1}$ Calculations were performed by E. van Heumen of the University of Amsterdam.
} 
spectra, states up to $\sim 50 \mathrm{meV}$ above the Fermi level are resolved due to thermal population. We took this $20 \mathrm{~nm}$ film measured in-situ, both at room and low temperature, out of the ARPES system and exposed it to air for 10 minutes. After this exposure, we loaded the sample again (no further treatments such as annealing of the sample) and reacquired ARPES data in order to assess the influence of air exposure on the band structure of our $\mathrm{Bi}_{2} \mathrm{Te}_{3}$ films. We can clearly see from Fig. 4.8(b) that bulk valence bands have moved downward after exposure and compared to the pristine sample, evident changes in the linearly dispersing Dirac surface states are observed. There are no surface states resolved after exposure to air, implying that the film degradation is deeper than the probing depth of the ARPES experiment $(\sim 1 \mathrm{~nm})$. Thus, the conductance of the films will be influenced by this downward shift of bulk bands, and the corresponding upward shift of $E_{F}$. Recently, upward shift of $E_{F}$ in $B_{2} T_{e}$ films exposed to air has also been reported in ARPES study [9].

We now discuss the effect of exposure to air in STM/STS data. We took the previously in-situ measured $30 \mathrm{~nm} \mathrm{Bi} \mathrm{Te}_{3}$ film grown on sapphire out of the STM system and exposed it to air for 10 minutes. After this procedure, we acquired several topography and spectroscopy maps at different position on the exposed surface. We used the same setpoint parameters as for the spectra shown in Fig. 4.4(a). We observe once more clear changes in the general shape of the spectra (see Fig.4.9): most notably, the region previously associated with linearly dispersing surface bands is absent in the exposed sample. This observation is consistent with our ARPES data of the exposed $20 \mathrm{~nm}$ film (see Fig. 4.8(b)) since there also, a clear degradation of the Dirac surface states was observed. Based on a comparison of measured STM topographies before and after exposure, we find indications that extrinsic defects due to contamination are responsible for the observed degradation of the surface states in the spectroscopic measurements. Figure 4.8 (c) shows atomically-resolved STM topography image of a $30 \mathrm{~nm} \mathrm{Bi} \mathrm{Te}_{3}$ film after exposure to ambient conditions, in which additional defects can be readily identified. On top of the observed defects and the observed disappearance of surface states, also shifts in the position of the bulk bands due to band bending are observed in the STS spectra (see Fig. 4.9). Whether bands shift upward or downward might sensitively depend on the exact exposure conditions as well as the position where spectra are taken (e.g. close to a defect). Nonetheless, from the combined ARPES and STS spectroscopic data it is clear that exposure to ambient pressures changes notably the surface states of our $\mathrm{Bi}_{2} \mathrm{Te}_{3}$ thin films. These results underline the need of protecting films before taking them ex-situ for further studies, since extrinsic defects may be responsible for the change of sign of the carriers in the surface conductivity of Tls [44]; for the deterioration of the topological surface state properties [19] and the reduction of the spin polarization [45].

The XPS surface analysis of the exposed sample reveals a splitting of the Te $3 d_{3 / 2}$ and $\mathrm{Te} 3 d_{5 / 2}$ 


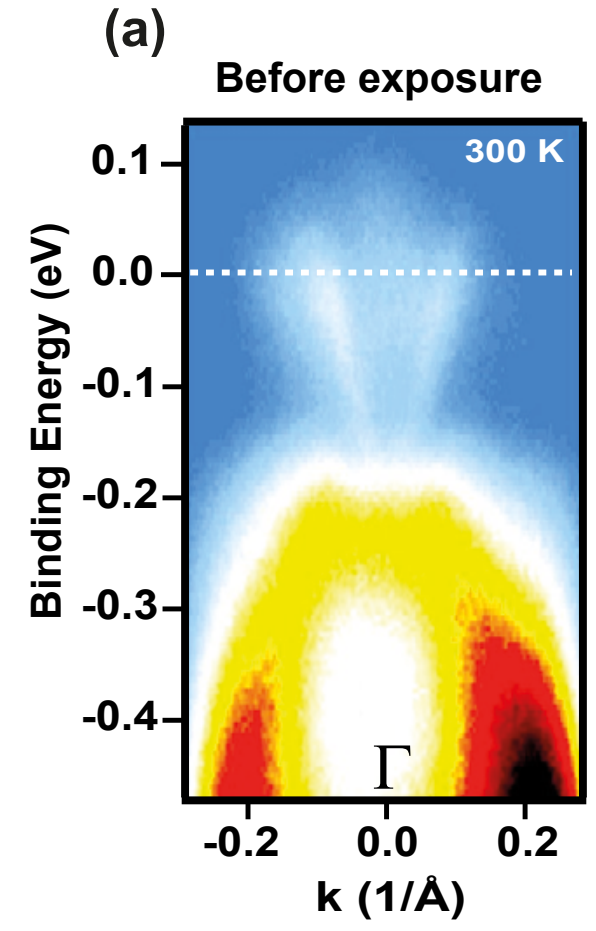

\section{(b)}
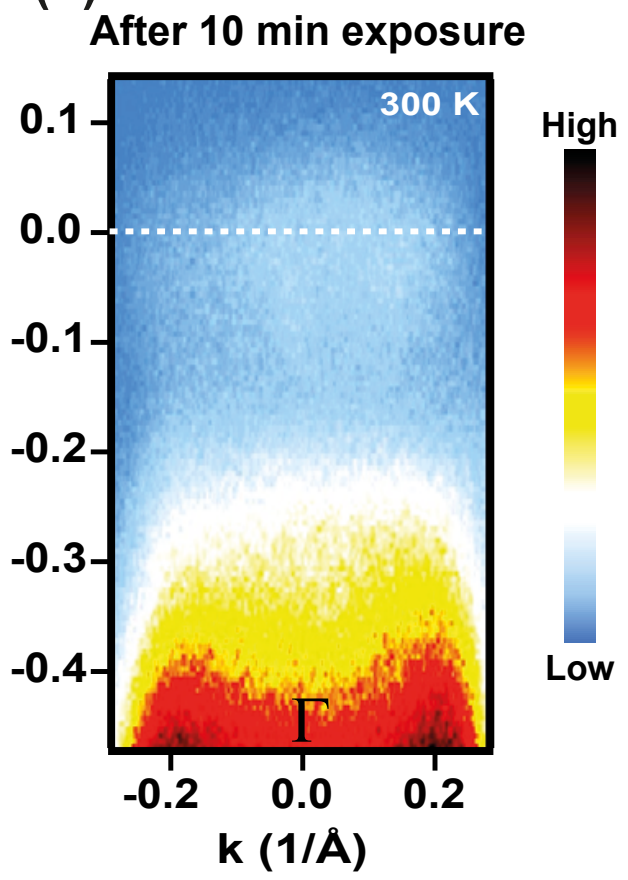

(c)

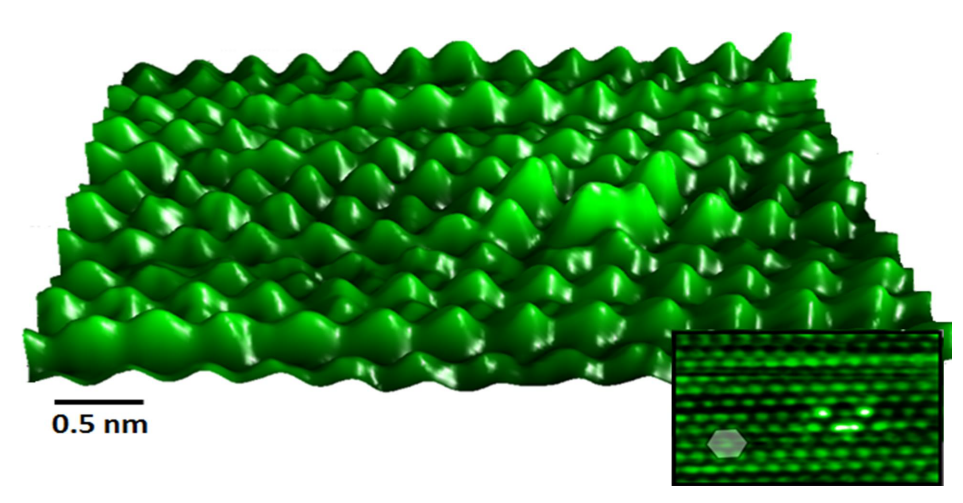

Figure 4.8: Effect of air exposure under ambient conditions on spectroscopy and topography of $\mathrm{Bi}_{2} \mathrm{Te}_{3}$ films grown on sapphire. (a) ARPES spectra around the $\Gamma$ point of a $20 \mathrm{~nm}$ film before and (b) after 10 minutes exposure to atmospheric pressure. (c) STM topography $\left(6 \times 3.5 \mathrm{~nm}^{2}\right)$ of a $30 \mathrm{~nm}$ film after exposure to ambient conditions. The surface tellurium atoms with a hexagonal crystal structure (right bottom insert) are still resolved together with noticeable defects as compared to before exposure (same sample as in Fig. 4.3(c)) to ambient conditions.

peaks consistent with the formation of tellurium oxide [46] $\left(\mathrm{TeO}_{2}\right)$, see Fig. 4.10(b)-(c). Since it was already shown that only pure oxygen doesn't affect topological surface states (see above in section 4.3 and Ref. [9]) we believe that other molecules from atmosphere, most likely water vapour, are the origin of the observed contamination. This conclusion emphasizes that for further ex-situ electronic transport investigations and quantum device fabrication, films should first be 


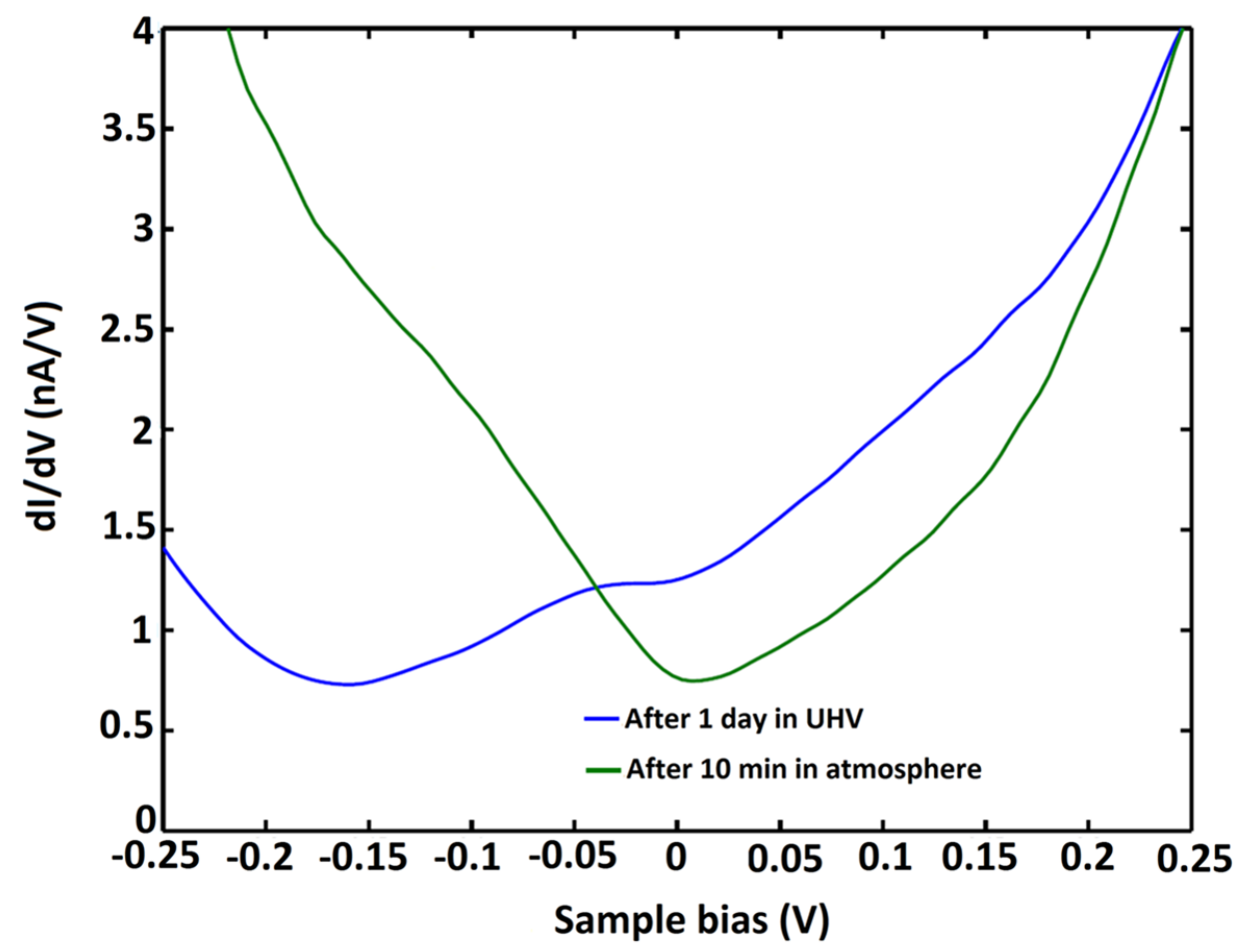

Figure 4.9: Conductance spectra of a film exposed for 10 min exposed to ambient (air) conditions. Before exposure (blue) and after exposure (green). The region previously associated with linearly dispersing surface bands is absent in the exposed sample, highlighting the deterioration of surface states after exposure to ambient conditions as observed in the ARPES data in Fig. 4.8(b) above.

capped in-situ to avoid any possible contamination as has already been indicated for $\mathrm{Bi}_{2} \mathrm{Se}_{3}$ thin films [48]. For $\mathrm{Bi}_{2} \mathrm{Te}_{3}$ films, though, a systematic study is needed in order to determine a suitable capping layer that can preserve the surface states and avoid unintentional doping induced by ambient conditions. An insulating layer would be appropriate since it will allow the fabrication of nearly damage-free top gate structures, so that the carriers in the top surface can be efficiently tuned [49]

\subsection{Conclusion and outlook}

In summary, we have systematically investigated the effect of in-situ exposure to pure oxygen at atmospheric pressure and of ex-situ contamination on high quality intrinsic $\mathrm{Bi}_{2} \mathrm{Te}_{3}$ films grown sapphire and STO. The XPS studies shows narrow and symmetric Te and Bi peaks highlighting the absence of $\mathrm{Te}$ or $\mathrm{Bi}$ excess in our films. STM confirms that the surface has atomically flat terraces and is free from intrinsic defects. The analysis of STS spectra underlines that the surface 
(a)

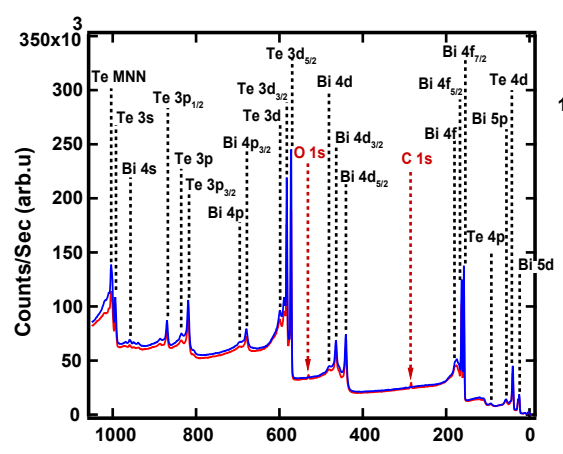

(b)

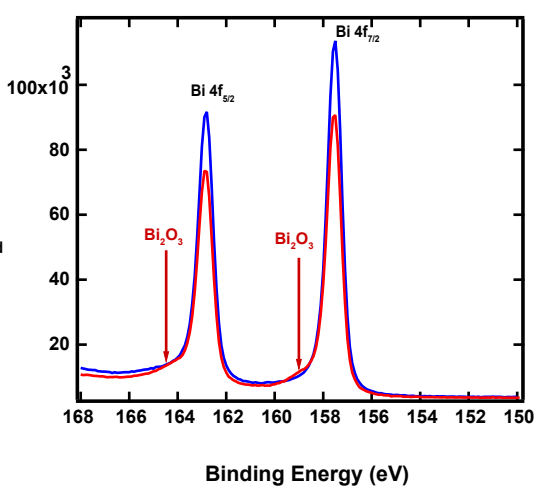

(c)

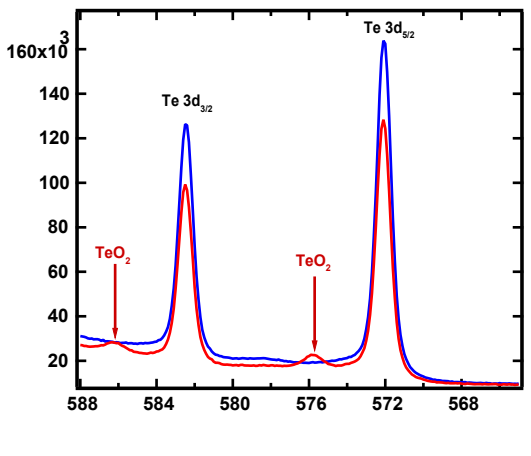

Figure 4.10: Effect of ex-situ exposure on a $30 \mathrm{~nm} \mathrm{Bi}_{2} \mathrm{Te}_{3}$ film grown on sapphire. (a) An XPS survey scan when the sample is measured in-situ (blue) and after being exposed to ambient conditions (red). After ex-situ exposure, oxygen and carbon peaks appear. High resolution Bi $4 f$ (b) and Te $3 d$ (c) XPS spectra before and after short exposure. After exposure, there is a clear formation of a $\mathrm{TeO}_{2}$ layer, whereas $\mathrm{Bi}_{2} \mathrm{O}_{3}$ formation is barely noticeable. This is consistent with the fact that the surface in an ideal crystal is terminated by tellurium atoms [2]. The bismuth oxidation would be expected to become significant, after the tellurium outer layer has been completely oxidized.

states of $\mathrm{Bi}_{2} \mathrm{Te}_{3}$ films are stable against oxidation and do not degrade as long as they are stored in-situ in ultra high vaccuum or in pure oxygen. ARPES data recorded from films grown in the same manner further confirms the presence of topological surface states with no bulk bands at the Fermi level. This study also provides a platform to investigate bottom gate tunable hybrid transport $[50,51]$ at the surface of intrinsic $\mathrm{Bi}_{2} \mathrm{Te}_{3}$ films grown on STO [111] substrates. The combined in- and ex-situ spectroscopy study reported here emphasizes the importance of capping these films before exposure to subsequent device fabrication steps. A possible route is to in-situ cap the $\mathrm{Bi}_{2} \mathrm{Te}_{3}$ and other $\mathrm{TI}$ films with an insulating $\mathrm{AIO}_{\mathrm{x}}$ layer. 


\section{Bibliography}

[1] L. Fu, C. L. Kane, and E. J. Mele, Phys. Rev. Lett. 98, 106803 (2007).

[2] H. Zhang, C. -X. Liu, X. -L Qi, X. Dai, Z. Fang, S. -C. Zhang, Nature Phys. 5, 438 (2009).

[3] J. E. Moore, Nature 464, 194 (2010).

[4] L. He, F. Xiu, X. Yu, M. Teague, W. Jiang, Y. Fan, X. Kou, M. Lang, Y. Wang, G. Huang, N. -C. Yeh, and K. L. Wang, Nano Lett. 12, 1486 (2012).

[5] A. A. Taskin, S. Sasaki, K. Segawa, and Y. Ando, Phys. Rev. Lett. 109, 066803 (2012).

[6] L. He, X. Kou, and K. L. Wang, Phys. Status Solidi RRL 7, 50 (2013).

[7] Y. Li, G. Wang, X. Zhu, M. Liu, C. Ye, X. Chen, Y. Wang, K. He, L. Wang, X. Ma, H. Zhang, X. Dai, Z. Fang, X. Xie, Y. Liu, X. Qi, J. Jia, S. Zhang, and Q. Xue, Adv. Funct. Mater. 22, 4002 (2010).

[8] G. Wang, X. Zhu, Y. Sun, Y. Li, T. Zhang, J. Wen, X. Chen, K. He , L. Wang, X. Ma, J. Jia, S. B. Zhang, and Q. Xue, Adv. Funct. Mater. 23, 2929 (2011).

[9] K. Hoefer, C. Becker, D. Rata, J. Swanson, P. Thalmeier, and L. H. Tjeng, Proc. Natl. Acad. Sci. USA 111, 14979 (2014).

[10] X. He, T. Guan, X. Wang, B. Feng, P. Cheng, L. Chen, Y. Li, and K. Wu, App. Phys. Lett. 101, 123111 (2012).

[11] H. M. Benia, C. Lin, K. Kern, and C. R. Ast, Phys. Rev. Lett. 107, 177602 (2011).

[12] C. Chen, S. He, H. Weng, W. Zhang, L. Zhao, H. Liu, X. Jia, D. Mou, S. Liu, J. He, Y. Peng, Y. Feng, Z. Xie, G. Liu, X. Dong, J. Zhang, X. Wang, Q. Peng, Z. Wang, S. Zhang, F. Yang, C. Chen, Z. Xu, X. Dai, Z. Fang, and X. J. Zhou, Proc. Natl. Acad. Sci. USA 109, 3694 (2012). 
[13] O. Eibl, K. Nielsch, N. Peranio and F. Völklein, Thermoelectric $B i_{2} T_{3}$ Nanomaterials, Wiley-VCH Verlag GmBh \& Co. KGaA, Weinheim, Germany (2015).

[14] J. Chang, Y.-S. Park, and S.-K. Kim, Appl. Phys. Lett. 92, 152910 (2008).

[15] G. Zhang, H. Qin , J. Chen , X. He , L. Lu, Y. Li , and K. Wu, Adv. Funct. Mater. 21, 2351 (2011).

[16] N. Bansal, Y. S. Kim, E. Edrey, M. Brahlek, Y. Horibe, K. lida, M. Tanimura, G. -H. Li, T. Feng, H. -D. Lee, T. Gustafsson, E. Andrei, and S. Oh, Thin Solid Films 520, 224 (2011).

[17] S. E. Harrison, S. Li, Y. Huo, B. Zhou, Y. L. Chen, and J. S. Harris, Appl. Phys. Lett. 102, 171906 (2013).

[18] H. Bando, K. Koizumi, Y. Oikawa, K. Daikohara, V. A. Kulbachinskii and H. Ozaki, J. Phys.: Condens. Matter 12, 5607 (2000).

[19] D. Kong, J. J. Cha, K. Lai, H. Peng, J. G. Analytis, S. Meister, Y. Chen, H. Zhang, I. R. Fisher, Z. Shen, and Y. Cui, ACS Nano 5, 4698 (2011).

[20] J. Guo, F. Qiu, Y. Zhang, H. Deng, G. Hu, X. Li, G. Yu and N. Dai, Chin. Phys. Lett. 30, 106801 (2013).

[21] L. V. Yashina, J. Sánchez-Barriga, M. R. Scholz, A. A. Volykhov, A. P. Sirotina, V. S. Neudachina, M. E. Tamm, A. Varykhalov,D. Marchenko, G. Springholz, G. Bauer, A. KnopGericke and O. Rader, ACS Nano 7, 5181 (2013).

[22] A. Roy, S. Guchhait, S. Sonde, R.Dey, T. Pramanik,A. Rai, H. C. P. Movva, L. Colombo, and S. K. Banerjee, Appl. Phys. Lett. 102, 163118 (2013).

[23] H.T. Zhang, X.G. Luo, C.H. Wang, Y.M. Xiong, S.Y. Li, X.H. Chen, J. Crys. Grow. 265, 558 (2004).

[24] J. Park, Y. -A Soh, G. Aeppli, S. R. Bland, X. -G. Zhu, X. Chen, Q. -K. Xue, and F. Grey, Appl. Phys. Lett. 101, 221910 (2012).

[25] A. Koma, Surf. Sci. 267, 29 (1992).

[26] A. Koma, Thin Solid Films 216, 72 (1992).

[27] G. Firpo and A. Pozzo, Rev. Sci. Instrum. 75, 4828 (2004).

[28] S. Urazhdin, D. Bilc, S. D. Mahanti, and S. H. Tessmer, Phys. Rev. B 69, 085313 (2004). 
[29] Z. Alpichshev, J. G. Analytis, J. -H. Chu, I. R. Fisher, Y. L. Chen, Z. X. Shen, A. Fang, and A. Kapitulnik, Phys. Rev. Lett. 104, 016401 (2010).

[30] M. Chen, J. Peng, H. Zhang, L. Wang, K. He, X. Ma, and Q. Xue, Appl. Phys. Lett. 101, 081603 (2012).

[31] To get differential conductivity spectra shown in this work, the sum of more than $3600 \mathrm{I-V}$ curves was numerically differentiated. The I-V spectra were also recorded by means of the standard Lock-in technique with a Lock-in time constant of $10 \mathrm{~ms}$. The Lock-in extracted $\mathrm{dl} / \mathrm{dV}$ spectra were consistent to the ones obtained with numerical differentiation.

[32] Y. L. Chen, J. G. Analytis, J. -H. Chu, Z. K. Liu, S. -K. Mo, X. L. Qi, H. J. Zhang, D. H. Lu, X. Dai, Z. Fang, S. C. Zhang, I. R. Fisher, Z. Hussain and Z. -X. Shen, Science 325, 178 (2009).

[33] F. Zahid and R. Lake, Appl. Phys. Lett. 97, 212102 (2010).

[34] T. Zhang, P. Cheng, X. Chen, J. F. Jia, X. Ma, K. He, L. Wang, H. Zhang, X. Dai, Z. Fang, X. Xie, and Q. -K. Xue, Phys. Rev. Lett. 103, 266803 (2009).

[35] P. Adroguer, D. Carpentier, J. Cayssol and E. Orignac, New J. Phys. 14, 103027 (2012).

[36] To perform this analysis, we added a probability factor of the form (see Ref. [37, 38] for details): $P \propto e^{2 z} \sqrt{C+k_{\|}^{2}}$, where $z$ is the tip-sample separation in the tunnelling experiment, and $C$ the height of the tunnelling barrier in units of $k^{2}$. Nonetheless, this improved fitting yielded insufficient result since the modelled kink due to warping was not strong enough to capture the measured kink feature in conductance spectra.

[37] A. van Houselt, T. Gnielka, M. Fischer, J. M. J. Aan de Brugh, N. Oncel, D. Kockmann, R. Heid, K. -P. Bohnen, B. Poelsema and H. J. W. Zandvliet, Surf. Sci. 602, 1731 (2008).

[38] H. J. W. Zandvliet and A. van Houselt, Annu. Rev. Anal. Chem. 2, 37 (2009).

[39] S. Basak, H. Lin, L. A. Wray, S.-Y. Xu, L. Fu, M. Z. Hasan, and A. Bansil, Phys. Rev. B 84, 121401(R) (2011).

[40] L. Miao, Z. F. Wang, W. Ming, M. -Y. Yao, M. Wang, F. Yang, Y. R. Songa, F. Zhu, A. V. Fedorovc, Z. Sund, C. L. Gao, C. Liu, Qi-Kun Xuee, C. -X. Liuf, F. Liub, D. Qian, and J. -F. Jia, Proc. Natl. Acad. Sci. USA 110, 2758 (2012).

[41] D. Culcer, E. H. Hwang, T. D. Stanescu, S. Das Sarma,. Phys. Rev. B 82, 155457 (2010). 
[42] B. Zhou, Z. K. Liu, J. G. Analytis, K. Igarashi, S. K. Mo, D. H. Lu, R. G. Moore, I. R. Fisher, T. Sasagawa, Z. X. Shen, Z. Hussain and Y. L. Chen, Semicond. Sci. Technol. 27, 124002 (2012).

[43] J. H. Hwang, J. Park, S. Kwon, J. S. Kim and J. Y. Park, Surf. Sci. 630, 153 (2014).

[44] A. A. Taskin, Z. Ren, S. Sasaki, K. Segawa, and Y. Ando, Phys. Rev. Lett. 107, 016801 (2011).

[45] X. Wang, G. Bian, T. Miller, and T. -C. Chiang, Phys. Rev. Lett. 108, 096404 (2012).

[46] To find the stoichiometry of the $\mathrm{TeO}_{x}$ layer, we fitted the oxidized XPS spectra using the same fitting procedure as for the XPS spectra in Fig. 4.1. The $\mathrm{TeO}_{x}$ peak was found at $576.01 \pm 0.05 \mathrm{eV}$, which is in agreement with the peak position of $\mathrm{TeO}_{2}$ given in the Handbook of x-ray photoelectron spectroscopy [47].

[47] J. F. Moulder, W. F. Stickle, P. E. Sobol, and K. D. Bomben, Handbook of X-ray Photoelectron Spectroscopy, Physical Electronics, Inc., Minnesota, USA (1995).

[48] J. Dai, W. Wang, M. Brahlek, N. Koirala, M. Salehi, S. Oh and W. Wu, Nano Research, 1-7 (2014), doi:10.1007/s12274-014-0607-8.

[49] F. Yang, A. A. Taskin, S. Sasaki, Ko. Segawa, Y. Ohno, K. Matsumoto, and Y. Ando, Appl. Phys. Lett. 104, 161614 (2014).

[50] C. Kurter, A. D. K. Finck, P. Ghaemi, Y. S. Hor, and D. J. Van Harlingen, Phys. Rev. B 90, 014501 (2014).

[51] B. Sacépé, J. B. Oostinga, J. Li, A. Ubaldini , N. J. G. Couto, E. Giannini and A. F. Morpurgo, Nat. Commun. 2, 575 (2011). 


\section{5}

\section{Gate-tunable transport properties of in-situ capped $\mathrm{Bi}_{2} \mathrm{Te}_{3}$ topological insulator thin films}

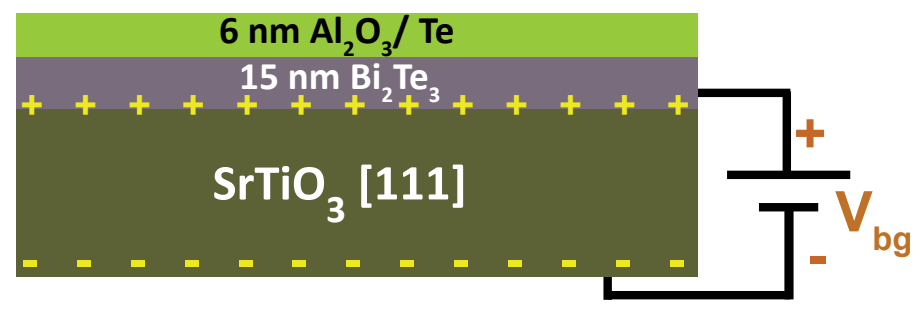

Combining the ability to prepare high-quality, intrinsic $\mathrm{Bi}_{2} \mathrm{Te}_{3}$ topological insulator thin films of low carrier density with in-situ protective capping, we demonstrate a pronounced, gate-tunable change in transport properties of $\mathrm{Bi}_{2} \mathrm{Te}_{3}$ thin films. Using a back-gate, the carrier density is tuned by a factor of $\sim 7$ in $\mathrm{Al}_{2} \mathrm{O}_{3}$ capped $\mathrm{Bi}_{2} \mathrm{Te}_{3}$ sample and by a factor of $\sim 2$ in $\mathrm{Te}$ capped $\mathrm{Bi}_{2} \mathrm{Te}_{3}$ films. We achieve full depletion of bulk carriers, which allows us to access the topological transport regime dominated by surface state conduction. When the Fermi level is placed in the bulk band gap, we observe the presence of two coherent conduction channels associated with the two decoupled surfaces. Our magnetotransport results show that the combination of capping layers and electrostatic tuning of the Fermi level provide a technological platform to investigate the topological properties of surface states in transport experiments and pave the way towards the implementation of a variety of topological quantum devices.

This chapter is published as: P. Ngabonziza, M. P. Stehno, H. Myoren, V. A. Neumann, G. Koster, and A. Brinkman, Adv. Electron. Mater. 2, 1600157 (2016). 
Page 82 Chapter 5. Gate-tunable transport properties of in-situ capped $\mathrm{Bi}_{2} \mathrm{Te}_{3}$ topological ...

\subsection{Introduction}

Three-dimensional (3D) topological insulators (Tls) such as $\mathrm{HgTe}, \mathrm{Bi}_{2} \mathrm{Se}_{3}, \mathrm{Bi}_{2} \mathrm{Te}_{3}, \mathrm{Sb}_{2} \mathrm{Te}_{3}$, and their alloys, are materials that are characterized by a narrow band gap in the electronic band structure while their surfaces host non-gapped spin-momentum locked states $[1,2,3]$. These helical surface states are topologically protected owing to the strong spin-orbit coupling in the material, and they mimic relativistic Dirac electrons $[1,2,3]$. Tls have generated much interest, not only as fundamentally new electronic states of matter, but also for their potential future technological applications in the fields of spintronics and quantum computation $[5,4,6]$.

Bismuth telluride $\left(\mathrm{Bi}_{2} \mathrm{Te}_{3}\right)$ is a $3 \mathrm{D} \mathrm{TI}$ material, which has been investigated extensively [7, $8,9,10,11,12,13,14]$. The conducting surface states of $\mathrm{Bi}_{2} \mathrm{Te}_{3}$ have been observed first using surface-sensitive techniques like angle-resolved photoemission spectroscopy (ARPES) [7] and scanning tunneling microscopy (STM), complemented by the scanning tunneling spectroscopy (STS) method [8]. Nevertheless, at present it remains challenging to find unambiguous evidence of surface state transport in experiments on $\mathrm{Bi}_{2} \mathrm{Te}_{3}$ single crystals or thin films. This is mainly because the charge transport is dominated by bulk conductivity due to residual carriers, which complicates the direct exploitation of the remarkable properties of the surface states.

Besides the successful use of counterdoping as a means to compensate bulk carriers in $\mathrm{TI}$ materials $[15,16]$, progress has also been made in synthesizing a new generation of high-quality, bulk-insulating $\mathrm{Bi}_{2} \mathrm{Te}_{3}$ thin films using molecular beam epitaxy $[9,10,11]$. We have been able to grow $\mathrm{Bi}_{2} \mathrm{Te}_{3}$ thin films on different insulating substrates (STO [111] and $\mathrm{Al}_{2} \mathrm{O}_{3}$ [0001]) with the Fermi level placed in the bulk band gap [9]. Combining different surface-sensitive in-situ characterization techniques, we demonstrated that the surface morphology and electronic band structure of $\mathrm{Bi}_{2} \mathrm{Te}_{3}$ are not affected by in-vacuo storage and exposure to oxygen, whereas major changes are observed when films are exposed to ambient conditions. Independently, combined in-situ four-point probe conductivity and angle resolved photoemission spectroscopy experiments revealed the bulk insulating properties of $\mathrm{Bi}_{2} \mathrm{Te}_{3}$ thin films with metallic surface states $[10,11]$. In the work of Hoefer et al. [10], the authors also demonstrated that short exposure to air causes the bulk conduction band to be filled with electrons and the conductivity is no longer determined by the surface states alone. These observations highlight the need to cap $\mathrm{Bi}_{2} \mathrm{Te}_{3}$ thin films in-situ before exposing them to air and other ex-situ contaminations in order to protect the surface states from degradation and unintentional doping.

Different studies employed epitaxially-grown amorphous layers of $\mathrm{Se} / \mathrm{Te}[12,13]$ or an evaporated layer of $\mathrm{Al}[17]$ as a protective capping layer for $\mathrm{Bi}_{2} \mathrm{Te}_{3}$ thin films whereas others used $\mathrm{Al}_{2} \mathrm{O}_{3}$ grown 
with atomic layer deposition (ALD) [18]. However, it was noticed that the stoichiometry of the films was altered after removal of the Te/Se capping layer $[12,13]$ and that the ALD-grown $\mathrm{Al}_{2} \mathrm{O}_{3}$ layer can cause damage to the $\mathrm{Bi}_{2} \mathrm{Te}_{3}$ surface states [18]. Such damage decreases the surface mobility and generates a large number of impurity states which pin the surface chemical potential, a situation that will complicate an efficient electrostatic tuning of the Fermi level [18]. Only recently, elemental Te was identified as a suitable capping material for the protection of the topological surface of intrinsically insulating $\mathrm{Bi}_{2} \mathrm{Te}_{3}$ thin films [11], but systematic magnetotransport investigations of such capped films are needed. Further, an insulating capping layer is preferable because it allows for the fabrication of nearly damage-free top gate structures [19]. Thus, it would be much more advantageous to develop a method for growing insulating capping layers with minimal damage to the surface states of intrinsically insulating $\mathrm{Bi}_{2} \mathrm{Te}_{3}$ thin films.

On the other hand, as the materials issues are being solved, the focus should now turn to finding an efficient way of controlling the Fermi level inside the bulk band gap. An effective gate control of topological surface states is highly desired for investigations of electronic transport properties in $\mathrm{Bi}_{2} \mathrm{Te}_{3}$ thin films and related device applications $[17,18,20,21,22,23,24]$. To-date, uniform gate-modulation of the carrier density and chemical potential over large areas and wide ranges has not been demonstrated in (capped) $\mathrm{Bi}_{2} \mathrm{Te}_{3}$ samples. Our first demonstration of this capability in this work is an important step toward the implementation of large scale hybrid devices for quantum information or spintronics applications with $\mathrm{Bi}_{2} \mathrm{Te}_{3}$ thin films.

We report bottom-gating results on in-situ capped, intrinsically insulating $\mathrm{Bi}_{2} \mathrm{Te}_{3}$ topological insulator thin films. We follow the growth recipe presented in Ref. [9] in order to obtain TI thin films with low densities of intrinsic carriers. First, we focus on determining the proper in-situ capping procedure of our $\mathrm{Bi}_{2} \mathrm{Te}_{3}$ thin films grown on different insulating substrates, namely $\mathrm{Al}_{2} \mathrm{O}_{3}$ and $\mathrm{SrTiO}_{3}$. We use two different capping materials: an $\mathrm{Al}_{2} \mathrm{O}_{3}$ layer, grown by pulsed laser deposition (PLD), and an epitaxially grown Te layer. Secondly, we report a systematic magnetotransport characterization of the in-situ capped films by studying the bottom-gate dependence of the sheet resistance, $2 \mathrm{D}$ carrier density $\left(n_{2 \mathrm{D}}\right)$ and weak antilocalization behavior. Here, we used the $\mathrm{SrTiO}_{3}$ substrate as the back-gate dielectric. Lastly, we compare the transport characteristics of capped films with uncapped films of the same thickness and grown in the same conditions. Dual evidence from both, extracted carrier densities and weak antilocalization (WAL) phenomena, suggests that the Fermi level is in the bulk band gap for the in-situ capped films since capping prevents the formation of native oxide and isolates the film from different extrinsic defects $[9,10,11,25]$. The transport data on in-situ capped $\mathrm{Bi}_{2} \mathrm{Te}_{3}$ thin films suggest that we can use the back-gate to tune the Fermi level all the way from the bulk conduction band (BCB) into the topological surface transport regime, and further down into the bulk valence band (BVB). These data reveal 
Page 84 Chapter 5. Gate-tunable transport properties of in-situ capped $\mathrm{Bi}_{2} \mathrm{Te}_{3}$ topological ...

(a)

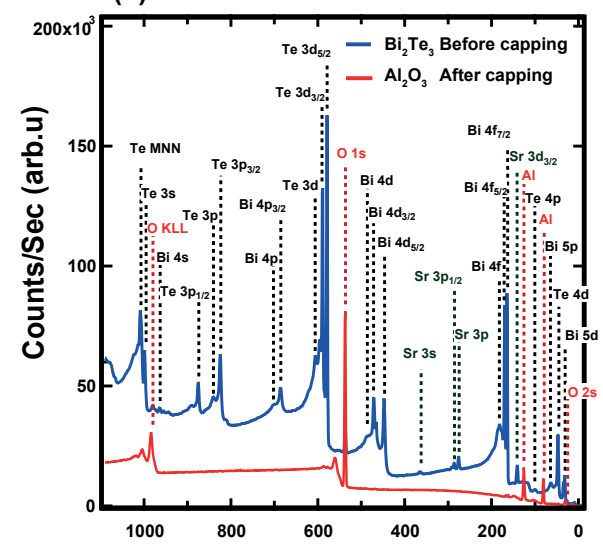

(b)

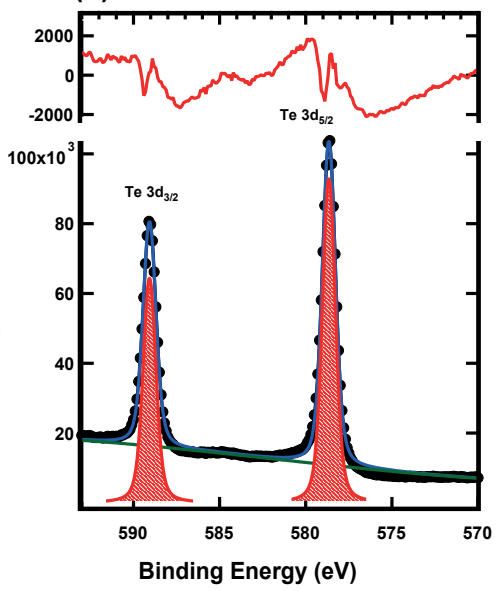

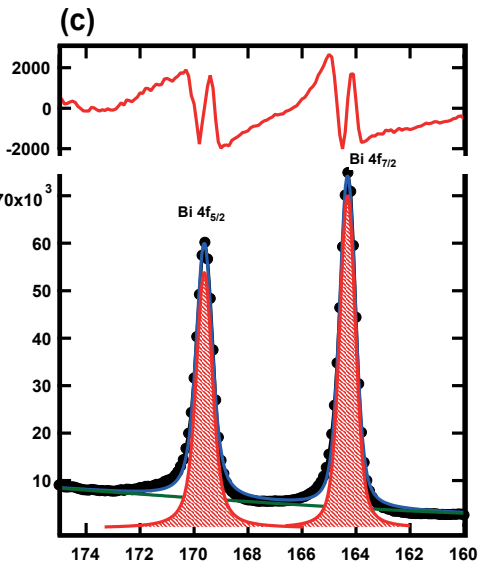

(d)

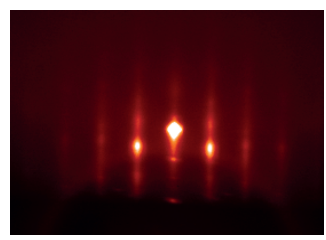

(e)

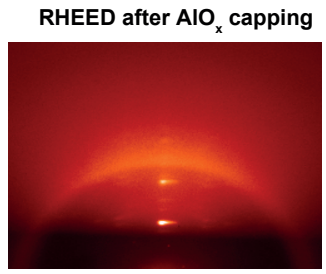

(f)

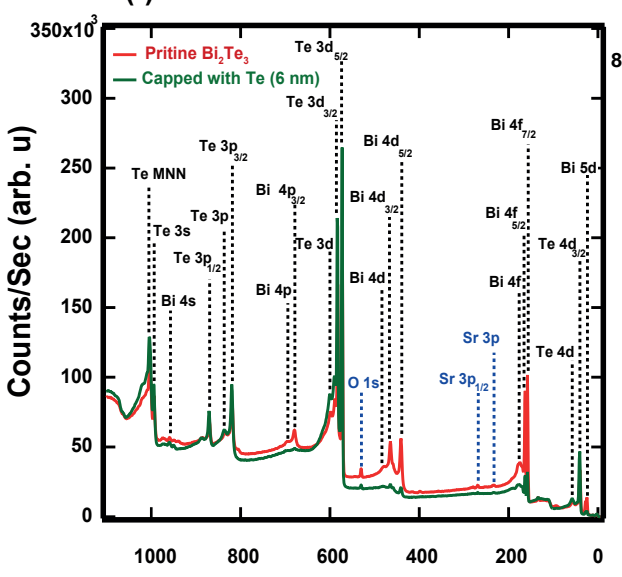

(g)

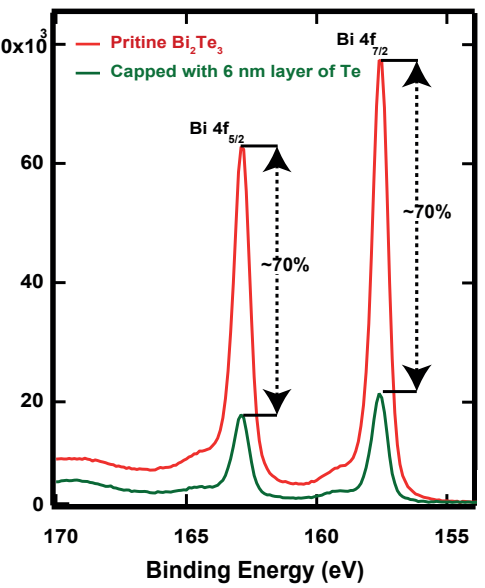

(h)

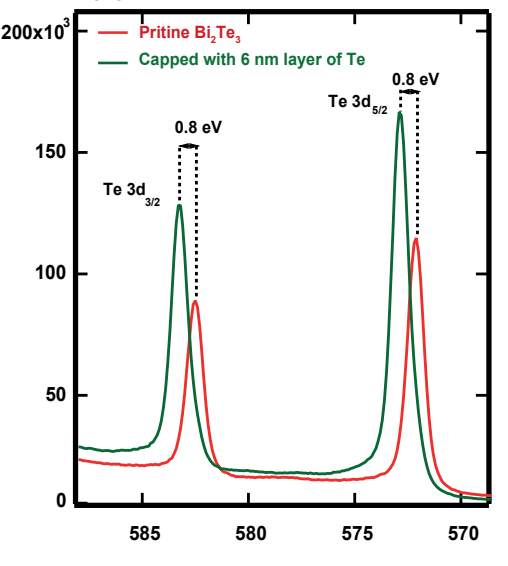

Figure 5.1: In-situ capping and surface characterizations of $15 \mathrm{~nm} \mathrm{Bi} \mathrm{Te}_{3}$ thin films. (a) An XPS survey scan taken after film growth (blue) and after capping with a $6 \mathrm{~nm} \mathrm{Al}_{2} \mathrm{O}_{3}$ layer (red). High-resolution scans around the $\mathrm{Te} 3 d$ (b) and $\mathrm{Bi} 4 f$ (c) main peaks, respectively, taken before capping in order to determine the surface stoichiometry. The red shaded zone shows the areas of the fitted peaks with the background (green line) removed. The upper red curve is the residual after Shirley background subtraction. RHEED patterns of the sample before (a) and after (b) insitu capping with an insulating layer of $\mathrm{Al}_{2} \mathrm{O}_{3}$ grown by pulsed laser deposition. (f) XPS spectra of pristine $\mathrm{Bi}_{2} \mathrm{Te}_{3}$ (red) and after capping with an epitaxially grown $6 \mathrm{~nm}$ layer of $\mathrm{Te}$ (green). High resolution scans of $\mathrm{Bi} 4 f(\mathrm{~g})$ and $\mathrm{Te} 3 d(\mathrm{~h})$ core levels measured before and after capping. 
that capping the sample before taking it ex-situ for further electronic transport studies helps in preserving the surface states and avoiding unintentional doping induced by ambient conditions.

\subsection{In-situ surface characterization}

The high-quality $\mathrm{Bi}_{2} \mathrm{Te}_{3}$ thin films used in this work were grown by molecular-beam epitaxy (MBE) on insulating substrates, $\mathrm{STO}[111]$ and $\mathrm{Al}_{2} \mathrm{O}_{3}[0001]$. The growth procedure was similar to Ref. [9]. Here, we compare magnetotransport properties of two samples of $15 \mathrm{~nm}$ thickness grown on STO. One sample was capped with amorphous $\mathrm{Al}_{2} \mathrm{O}_{3}$ and the other with an epitaxiallygrown Te layer. The thickness of the protective capping layers of either material was $\sim 6 \mathrm{~nm}$. For the purpose of understanding the role of capping, control samples without the protective layer were grown under the same conditions for comparison. Before and after capping, the surface condition of films were checked using X-ray photoemission spectroscopy (XPS) and reflection high energy electron diffraction (RHEED). For in-situ surface elemental characterization and detection of any $\mathrm{Te} / \mathrm{Bi}$ excess in our films, we used XPS. Figure 5.1(a) shows the XPS spectra of the pristine (blue) film. Only the $\mathrm{Bi}$ and $\mathrm{Te}$ peaks are resolved with no appearance of extra peaks, confirming that the film is clean without contaminations. The analysis of the high resolution scans around the $\mathrm{Te} 3 d$ and $\mathrm{Bi} 4 f$ peaks for the pristine $\mathrm{Bi}_{2} \mathrm{Te}_{3}$ film (see Fig. 5.1(b) and (c)) reveal narrow and symmetric core level lines, signaling the absence of $\mathrm{Te}$ or $\mathrm{Bi}$ excess in our film. The surface chemical stoichiometry was determined following the procedure presented in Ref. [9] and the Te:Bi ratio was determined to be $1.497 \pm 0.05$. Figure $5.1(\mathrm{~d})$ shows a typical RHEED pattern of the pristine $\mathrm{Bi}_{2} \mathrm{Te}_{3}$ film before being capped with an $\mathrm{Al}_{2} \mathrm{O}_{3}$ layer. The sharp RHEED streaks are indicative of a high-quality film with smooth single crystalline domains. After depositing the $\mathrm{Al}_{2} \mathrm{O}_{3}$ layer by pulsed laser deposition (PLD), the RHEED pattern of the same sample is shown in Fig. 5.1(e) for comparison. For the deposition, the films had been transferred to the PLD chamber without breaking high vacuum (HV) conditions as the PLD system is connected to the growth chamber via an HV distribution chamber. The capping layer was deposited at room temperature in argon flow (40 sccm) at a background pressure of $1 \times 10^{-1}$ mbar using a laser energy density of $2.75 \mathrm{~J} / \mathrm{cm}^{2}$. In order to minimize the degradation of the $\mathrm{Bi}_{2} \mathrm{Te}_{3}$ surface states due to high energy ionic bombardment of the sample surface during the PLD process, the sample holder was placed at a relatively far distance $(\sim 60 \mathrm{~mm})$ from the target material. After the $\mathrm{Al}_{2} \mathrm{O}_{3}$ deposition, only the oxygen and aluminum peaks were resolved in the XPS spectrum (red curve in Fig. 5.1(a)).

For samples capped with Te, the capping was also performed at room temperature, immediately 
Page 86 Chapter 5. Gate-tunable transport properties of in-situ capped $\mathrm{Bi}_{2} \mathrm{Te}_{3}$ topological ...

after the growth of $\mathrm{Bi}_{2} \mathrm{Te}_{3}$ thin films in the MBE growth chamber without breaking the ultra-high vacuum conditions. The surface elemental composition of these films was also investigated using XPS. Only $\mathrm{Bi}$ and Te peaks are resolved in the XPS spectra before and after the deposition of the $6 \mathrm{~nm}$ Te capping layer (see Fig. 5.1(f)). The intensities of the Bi core levels in $\mathrm{Bi}_{2} \mathrm{Te}_{3}$ are observed to decrease after the capping step. From the analysis of the high resolution XPS spectra (see Fig. 5.1(g) and $(\mathrm{h})$ ), the $\mathrm{Bi} 4 f$ spectral intensity is determined to have reduced by $\sim 70 \%$ without any changes in the lineshape or energy position. This indicates that the $\mathrm{Bi}$ in $\mathrm{Bi}_{2} \mathrm{Te}_{3}$ is not chemically affected by the deposited $6 \mathrm{~nm}$ capping layer of Te [11]. On the other hand, the $\mathrm{Te} 3 d_{5 / 2}$ and $\mathrm{Te} 3 d_{3 / 2}$ peaks are slightly influenced by this capping procedure since their lineshape peak positions are shifted by $\sim 0.8 \mathrm{eV}$ toward higher binding energies upon the deposition of the Te overlayer. This shoulder has been observed previously and was attributed to elemental Te $[11,26]$. The XPS data on Te capped film are in agreement with recent in-situ ARPES and four-point probe conductivity data on $\mathrm{Bi}_{2} \mathrm{Te}_{3}$ films [11], which revealed that the surface states of the pristine $\mathrm{Bi}_{2} \mathrm{Te}_{3}$ samples are not affected by the Te capping, thus suggesting the Te capping method does not cause a doping of the sample.

\subsection{Gate-tunable transport characteristics}

In order to investigate the electronic transport properties of capped films and to study the effect of the protective capping layer in magnetransport properties of in-situ capped and uncapped $\mathrm{Bi}_{2} \mathrm{Te}_{3}$ thin films, we fabricated Hall bar devices using standard photolithography methods and subsequent wet etching. For the etching step, we used a solution of nitric acid $\left(\mathrm{HNO}_{3}\right)$ diluted with de-ionized water $\left(1 \mathrm{HNO}_{3}: 3 \mathrm{H}_{2} \mathrm{O}\right)$. Gold contacts were added in a subsequent lithography step. To achieve proper electric contact in the $\mathrm{Al}_{2} \mathrm{O}_{3}$ capped samples, we removed the insulating layer in the contact area by etching the aluminum oxide with the same developer solution (a base) that we use for photolithography. Figure 5.2(a) shows an optical image of a typical Hall bar device. We used the STO [111] substrate as the back-gate dielectric since it has a high dielectric constant at low temperature $(\sim 24000)$. The same approach has already been used for $\mathrm{Bi}_{2} \mathrm{Se}_{3}$ and $\left(\mathrm{Bi}_{0.5} \mathrm{Sb}_{0.5}\right)_{2} \mathrm{Te}_{3}$ thin films, and $\mathrm{Bi}_{2} \mathrm{Te}_{3}$ nanoribbons also using STO [111] as the back-gate dielectric $[22,27,28,29,30,31]$. Figure 5.2(b) depicts a schematic illustration of the bottom-gated thin film. Electrical transport properties of the films were first characterized in the temperature range from $300 \mathrm{~K}$ down to $2 \mathrm{~K}$ using a Quantum Design PPMS measurement system. Later, we conducted magnetotransport measurements at different back-gate voltages in a dilution refrigerator at $100 \mathrm{mK}$. The measurements were performed using a standard lock-in technique with a small excitation current of $5 \mathrm{nA}$. Both, the sheet resistance $R_{\mathrm{S}}$ and the Hall 
(a)

(b)

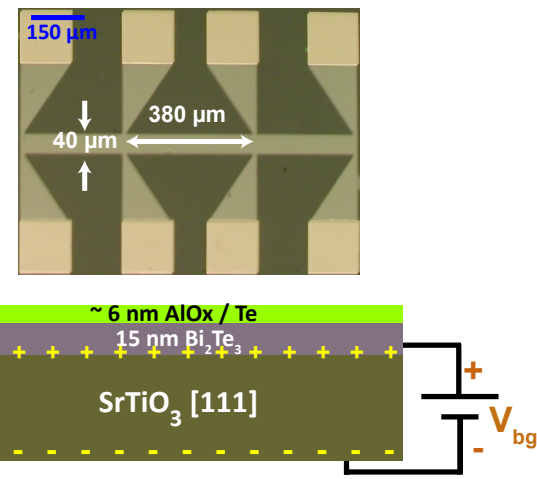

(d)

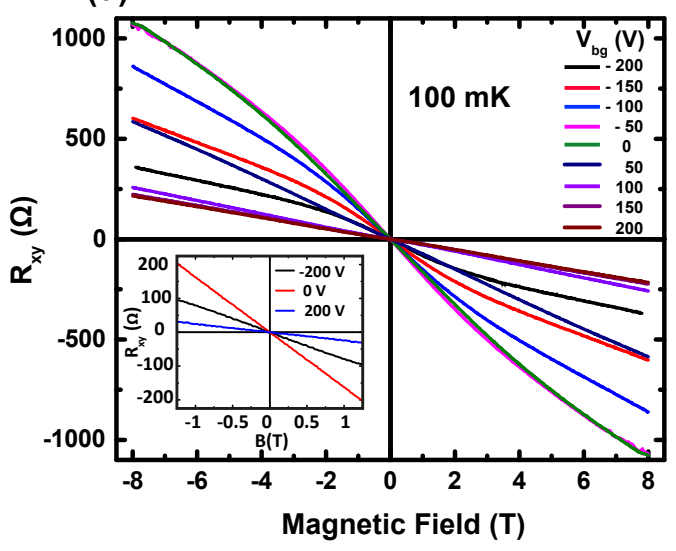

(c)

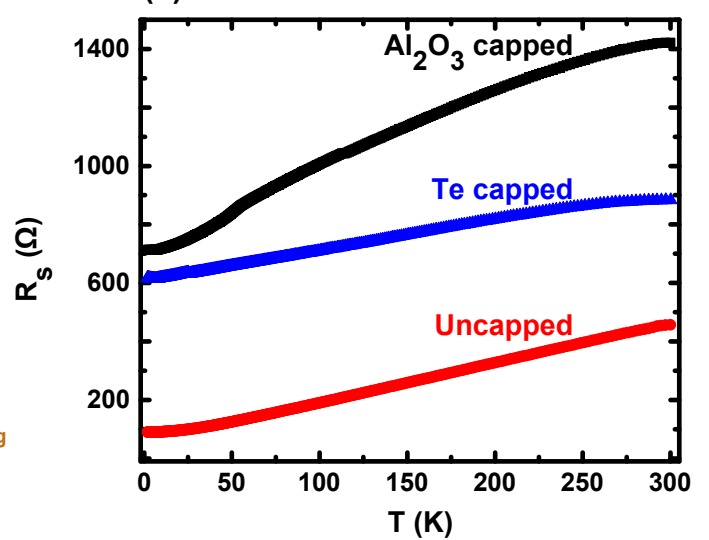

(e)

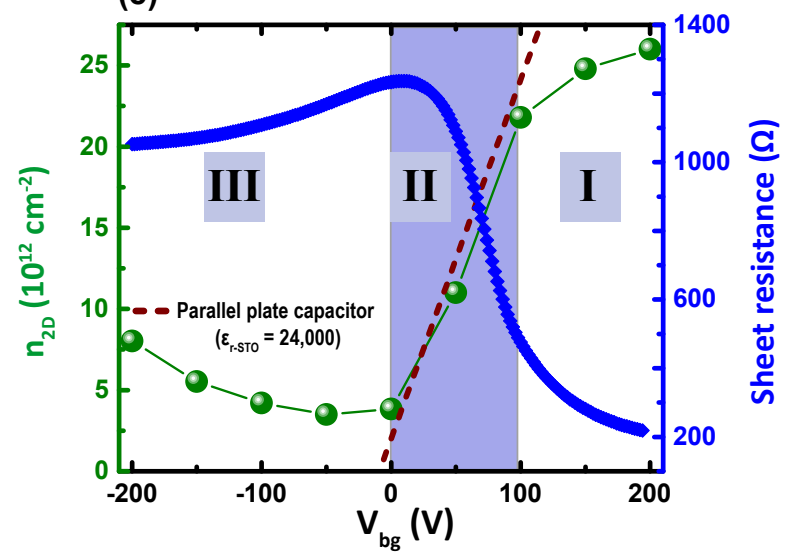

Figure 5.2: Transport characteristics of $15 \mathrm{~nm} \mathrm{Bi} \mathrm{Te}_{3}$ thin films. (a) Optical micrograph of the structured Hall bar device. (b) Schematic representation of a typical in-situ capped $\mathrm{Bi}_{2} \mathrm{Te}_{3}$ film grown on $\mathrm{SrTiO}_{3}$ [111]. The substrate is used as the back-gate dielectric in transport experiments. The total thickness of the capping layers (of either $\mathrm{Al}_{2} \mathrm{O}_{3}$ or $\mathrm{Te}$ ) is $\sim 6 \mathrm{~nm}$. (c) Temperature dependence of the zero-field sheet resistance for three different $\mathrm{Bi}_{2} \mathrm{Te}_{3}$ films. Black: capped with $\mathrm{Al}_{2} \mathrm{O}_{3}$, blue: capped with Te and red: uncapped film. (d) Back-gate voltage dependence of the Hall resistance, $R_{\text {xy }}$ at $100 \mathrm{mK}$. (e) Back-gate voltage $\left(V_{b g}\right)$ dependence of the 2D carrier density extracted from the Hall zero-field slope of $R_{x y}$ in (d) and of the sheet resistance. Three different gate voltage regions I, II and III are identified with distinct behavior. The change in 2D carrier density follows the parallel plate capacitor law in the intermediate $V_{b g}$ regime (dashed line fit in region $\mathrm{II})$.

resistance $R_{\mathrm{xy}}$, were measured as a function of magnetic field at different back-gate voltages $\left(V_{b g}\right)$, in the range of $\pm 200 \mathrm{~V}$. Sweeping the gate voltage back and forth, we observed hysteretic behavior, probably due to the presence of impurity states that trap charges as has been suggested by previous studies $[19,22,32]$. In order to keep consistency in the data, all graphs presented 
Page 88 Chapter 5. Gate-tunable transport properties of in-situ capped $\mathrm{Bi}_{2} \mathrm{Te}_{3}$ topological ...

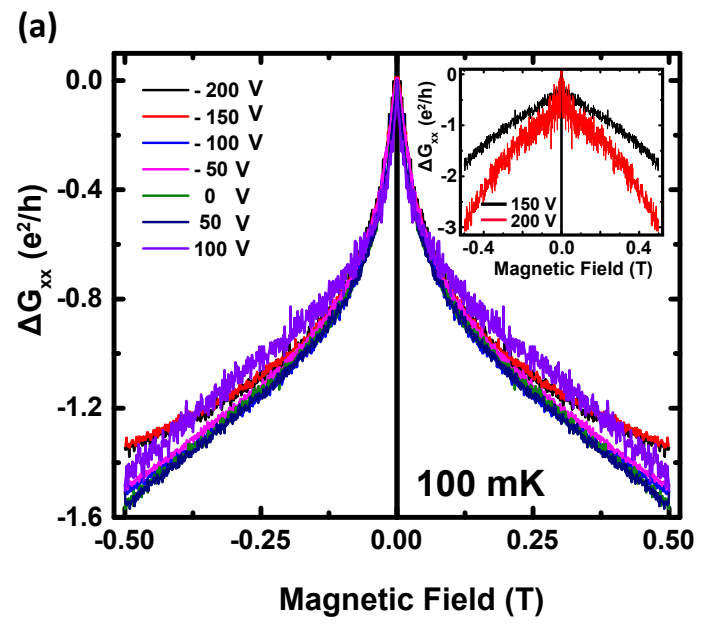

(b)

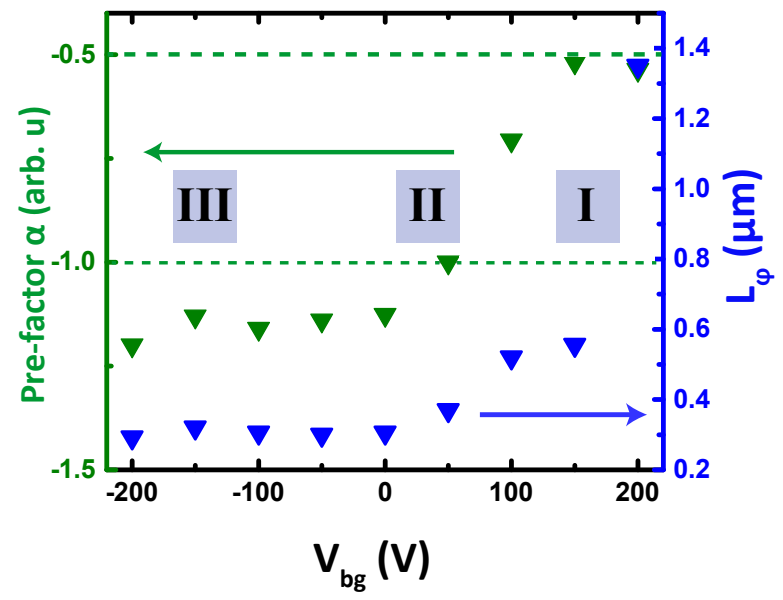

(c)

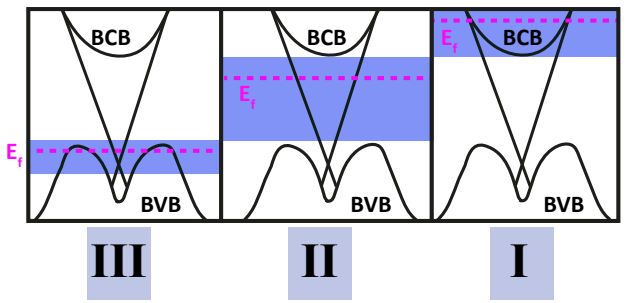

Figure 5.3: Back-gate dependence of the WAL effect of an in-situ $\mathrm{Al}_{2} \mathrm{O}_{3}$-capped $\mathrm{Bi}_{2} \mathrm{Te}_{3}$ thin film. (a) The gate-voltage dependence of the low field magnetoconductance $\Delta G_{\mathrm{xx}}(B)$. All data were taken at $100 \mathrm{mK}$. The inset highlights the observation of a parabolic background contribution to $\Delta G_{x x}$ at high positive $V_{b g}$. (b) Gate dependence of the prefactor $\alpha$ and the dephasing length $l_{\varphi}$, obtained from best fits to equation (5.1). (c) A schematic band diagram illustration relevant to the thin films used in this study. BCB is the bulk conduction band, BVB is the bulk valence band, and $E_{f}$ is the position of the Fermi level. We access the transport regimes I, II and III of (b) as the Fermi level moves through the respective shaded regions of the band structure in (c) and Fig. 5.2(e) when the gate voltage is lowered.

here were taken by sweeping $V_{b g}$ from positive to negative values.

Figure 5.2(c) presents the temperature-dependent zero magnetic field sheet resistance for two different capped $\mathrm{Bi}_{2} \mathrm{Te}_{3}$ films (black: capped with $\mathrm{Al}_{2} \mathrm{O}_{3}$, blue: capped with $\mathrm{Te}$ ), and for uncapped film (red curve). All three samples show metallic behaviour over the entire temperature range; but the sheet resistance is lower in the uncapped film by about $500 \Omega$ and $600 \Omega$ at $2 \mathrm{~K}$ compared to the Te and $\mathrm{Al}_{2} \mathrm{O}_{3}$-capped films, respectively. This observation is consistent with recent insitu ARPES and four probe STM transport measurements on pristine, capped and exposed to air $\mathrm{Bi}_{2} \mathrm{Te}_{3}$ thin films $[10,11]$. The decrease in $R_{\mathrm{S}}$ was attributed to originate from exposing films to ambient conditions; and corresponding ARPES spectra on the same pristine and capped 
films proved that the Fermi level is inside the bulk band gap prior and after in-situ transport measurements, which always showed metallic behaviour of the $\mathrm{Bi}_{2} \mathrm{Te}_{3}$ thin films $[10,11]$.

\subsubsection{Back-gate dependence of in-situ $\mathrm{Al}_{2} \mathrm{O}_{3}$-capped $\mathrm{Bi}_{2} \mathrm{Te}_{3}$ film}

We first discuss the magnetotransport properties of the $\mathrm{Al}_{2} \mathrm{O}_{3}$-capped $\mathrm{Bi}_{2} \mathrm{Te}_{3}$ thin film. The slope of $R_{\text {xy }}$ indicates $n$-type carriers. It increases with decreasing $V_{b g}$. The $R_{\text {xy }}$ curves start to show nonlinearity below $V_{b g}=50 \mathrm{~V}$ (see Fig. 5.2(d)). Similar behavior has been reported in previous work on $\mathrm{Bi}_{2} \mathrm{Se}_{3}$ and $\left(\mathrm{Bi}_{1-x} \mathrm{Sb}_{\times}\right)_{2} \mathrm{Te}_{3}$ thin films grown on $\mathrm{SrTiO}_{3}$ [111] substrate [22, 27, 28, 29]. We do not observe a reversal of the sign of $R_{\mathrm{xy}}$ in the entire magnetic field range in contrast to, e.g., $\left(\mathrm{Bi}_{1-x} \mathrm{Sb}_{\mathrm{x}}\right)_{2} \mathrm{Te}_{3}$ samples in Ref. [22]. In the case of $\mathrm{Bi}_{2} \mathrm{Se}_{3}$ thin films grown on STO [111], the change of the sign in the Hall signal has only been achieved at much higher magnetic fields and for large negative back-gate voltages $[27,28]$. We conjecture that the ambipolar effect is not observed within the accessible back-gate field range because of the "M-like" shape of the bulk valence band (BVB) structure of $\mathrm{Bi}_{2} \mathrm{Te}_{3}[7,9]$ (see Fig. 5.3(c) and related discussion below). The Dirac point (DP) is buried inside the BVB, thus only $n$-type topological surface states carriers are accessible inside the bulk band gap, as it has also been recently suggested from gate-tunable transport studies of $\mathrm{Bi}_{2} \mathrm{Te}_{3}$ nanoribbon [30].

The nonlinearity in the Hall resistivity curves below $50 \mathrm{~V}$ suggests the coexistence of multiple charge carrier types. We extracted the low-field Hall coefficients $\left(R_{H}\right)$ which are calculated from the slope of the $R_{x y}$ curves for $B<1.5 \mathrm{~T}$ (see inset Fig. 5.2(d)). It should be emphasized here that, in the case where $R_{x y}(\mathrm{~B})$ is nonlinear, a multiple band model approach should be taken. However, the total carrier density from a multiband fit and the carrier density from the low-field Hall coefficient do not differ much. For example, at $V_{b g}=0 \mathrm{~V}$, we obtain $4.2 \times 10^{12} \mathrm{~cm}^{-2}$ for the total carrier density in a two-band fit and $3.8 \times 10^{12} \mathrm{~cm}^{-2}$ from the low field Hall coefficient. We were not able to obtain a simultaneous fit to both components of the magnetoresistance data, $R_{\mathrm{xx}}$ and $R_{\mathrm{xy}}$, within the framework of the standard two-band model. A full theory of band structure and quantum corrections to the conduction of $3 \mathrm{D}$ TI surface and bulk bands is still missing. Similar observation was also pointed out in a recent review article on TI materials [33]. Thus, the $2 \mathrm{D}$ carrier density $\left(n_{2 D}\right)$ is extracted from $n_{2 D}=\frac{1}{e R_{H}}$, with the low-field Hall coefficient $R_{H}$ as defined above, and $e$ the electronic charge. The mobility is calculated using $\mu=\frac{1}{e R_{s} n_{2 D}}$ at zero magnetic field.

The response of sheet carrier density and sheet resistance of the $\mathrm{Al}_{2} \mathrm{O}_{3}$-capped $\mathrm{Bi}_{2} \mathrm{Te}_{3}$ thin film to the applied back-gate voltage is summarized in Fig. 5.2(e). The first notable observation is the appearance of a maximum in the sheet resistance at $\mathrm{V}_{b g} \sim 10 \mathrm{~V}$ (region II), which is the first 
Page 90 Chapter 5. Gate-tunable transport properties of in-situ capped $\mathrm{Bi}_{2} \mathrm{Te}_{3}$ topological ...

time, to our knowledge, that this behavior is seen in $\mathrm{Bi}_{2} \mathrm{Te}_{3}$. Previously, it has only been observed in $\mathrm{Bi}_{2} \mathrm{Se}_{3}$ and $\left(\mathrm{Bi}_{1-x} \mathrm{Sb}_{\mathrm{x}}\right)_{2} \mathrm{Te}_{3}$ single crystals and thin films [22, 27, 28, 29, 32, 34], and $\mathrm{Bi}_{2} \mathrm{Te}_{3}$ nanoribbons placed on $\mathrm{SrTiO}_{3}$ substrate [30]. However, we would like to point out that the resistance maximum is not correlated with the depletion of the surface states at the Dirac point (DP), сp. Refs. [27, 28, 29, 34], as the DP is buried below the BVB maximum. We suggest that the downturn and saturation of the sheet resistance at negative gate voltages (region III) is similar to what was observed in the measurements by Lee et al. [34] on $\mathrm{Bi}_{1.5} \mathrm{Sb}_{0.5} \mathrm{Te}_{1.7} \mathrm{Se}_{1.3}$ single crystals. The results were interpreted as an increase in interband scattering when the Fermi level touches the bulk valence band (see region III in Fig. 5.3(c)).

In total, we modulate the carrier density $n_{2 \mathrm{D}}$ by a factor of $\sim 7$ in the accessible back-gate voltage range $( \pm 200 \mathrm{~V})$. The biggest drop in carrier density is seen in region II of Fig. 5.2(e). The lowest extracted carrier density is $n_{2 \mathrm{D}}=3.5 \times 10^{12} \mathrm{~cm}^{-2}$ at $V_{b g} \sim-50 \mathrm{~V}$ with a corresponding mobility of $1,600 \mathrm{~cm}^{2} V^{-1} \mathrm{~s}^{-1}$. The low carrier concentration in the intermediate region strongly suggests that the Fermi level is located in the bulk band gap, crossing only topological surface states. This is further corroborated by weak antilocalization measurements presented later. Furthermore, $n_{2 \mathrm{D}}$ versus $V_{b g}$ is observed to be linear as one would expect from a parallel plate capacitor model (dashed line fit in Fig. 5.2(e)). All added carriers participate in electric conduction in the intermediate $V_{b g}$ regime, a behavior which is expected for surface states. Starting at $V_{b g}$ $=0 \mathrm{~V}$, when the $V_{b g}$ is increased further, we add carriers into the sample, most likely to the bottom surface first. Once the bottom surface reaches the bulk conduction band, we start to fill bulk states into the sample. This might even happen earlier since the bulk screening length is estimated to be in the range of 10 to $30 \mathrm{~nm}[35,36,37]$, which is in the same order of magnitude as our sample thickness $(15 \mathrm{~nm})$.

At large, positive and at negative back-gate voltages, the linear relationship does not hold. In region III, the carrier density starts to increase slowly again as the bulk valence band contributes mobile carriers. The Dirac point is buried deeper in the bulk valence band in $\mathrm{Bi}_{2} \mathrm{Te}_{3}$ samples which makes it unaccessible by electrostatic gating in the gate voltage range of our experimental setup $[7,9,10]$. In region I, we add fewer high-mobility carriers by increasing the gate voltage. We attribute this to the onset of bulk conduction when the Fermi level crosses the bottom of the bulk conduction band (BCB).

Additional information about the carrier distribution and the scattering between transport channels is obtained from a measurement of the weak (anti-)localization effect [38]. The WAL effect in TIs has been studied intensively both theoretically [39, 40] and experimentally [19, 28, 29, 41]. WAL is a quantum effect that is commonly observed in Tls due to the spin-momentum locking resulting from strong spin-orbit coupling [42]. The $\pi$-Berry's phase of the topological surface states 
leads to destructive interference between time-reversed charge carrier paths, thus increasing the conductivity at zero magnetic field. The cusp-like WAL maximum can be destroyed by applying an external magnetic field to the sample, which breaks the time reversal symmetry and $\pi$-Berry's phase $[39,42]$. Figure 5.3(a) gives the sheet conductance $\Delta G_{\mathrm{xx}}(B)=G_{\mathrm{xx}}(B)-G_{\mathrm{xx}}(B=0 \mathrm{~T})$ as a function of magnetic field for various $V_{b g}$ measured at $100 \mathrm{mK}$. The $\Delta G_{\mathrm{xx}}(B)$ data show sharp cusp-like maxima at zero magnetic field, indicating the existence of the WAL. At high positive $V_{b g}$, the WAL effect is suppressed, containing parabolic background (see inset Fig. 5.3(a)). The suppression of WAL at high $V_{b g}$ can be due to either enhanced bulk conductivity when $E_{f}$ is located in the bulk or disorder induced electron-electron interactions in the system $[41,43]$. The former scenario is much more likely in our samples since it is consistent with the $n_{2 D}$ analysis presented in Fig. 5.2(e) and is similar to the behaviors reported previously in gating experiments [32, 44].

The WAL behaviour is described quantitatively using the simplified Hikami-Larkin-Nagaoka (HLN) equation $[38,45]$. In the limit of strong spin-orbit coupling $\left(\tau_{\varphi} \gg \tau_{s o}, \tau_{e}\right.$; where $\tau_{\varphi}$ is the dephasing time, $\tau_{s o}$ the spin-orbit scattering time, and $\tau_{e}$ the elastic scattering time), with a negligible Zeeman term, the magnetoconductance correction is given by:

$$
\Delta G_{\mathrm{xx}}(B)=\alpha \frac{e^{2}}{\pi h}\left[\Psi\left(\frac{1}{2}+\frac{B_{\varphi}}{B}\right)-\ln \left(\frac{B_{\varphi}}{B}\right)\right]
$$

where $\alpha$ is a parameter whose value reflects the number of conduction channels. $\Psi$ is the digamma function, $B_{\varphi}=\hbar / 4 e l_{\varphi}^{2}$ is the characteristic magnetic field with $l_{\varphi}^{2}=D \tau_{\varphi}$, which is the phase coherent length, and $D$ the diffusion constant. The relationship between the prefactor $\alpha$ and the number of conducting channels in equation (5.1) is essential in differentiating the nature of transport channels of Tls [40]. For each two dimensional conducting channel, a value of $\alpha=-0.5$ is expected, and if there are two independent 2D conducting channels, the prefactors $\alpha$ add up. An effective dephasing length $l_{\varphi}$ will replace the phase relaxation length of the individual channel $[34,40]$. Fitting magnetoconductance data at different $V_{b g}$ to equation (5.1), we observe a modulation of the prefactor $\alpha$ and of the dephasing length $l_{\varphi}$ as presented in Fig. 5.3(b). The values of $\alpha$ drop from $\sim-0.5$ at high, positive $V_{b g}$ (region I), to a value of $\sim-1$ in the intermediate regime at $V_{b g} \simeq 50 \mathrm{~V}$ (region II), and saturate at a value slightly smaller than -1 for negative back-gate voltages (region III). The same trend is observed in the $V_{b g}$ dependence of $l_{\varphi}$. For large gate voltage, $V_{b g}=+200 \mathrm{~V}$, the phase coherence is long, $l_{\varphi}=1.35 \mu \mathrm{m}$, but it reduces to $l_{\varphi} \simeq 292 \mathrm{~nm}$ when $V_{b g}=0 \mathrm{~V}$ and flattens out for negative gate voltages (region III). The extracted $l_{\varphi}$ is larger than the thickness of the sample $(15 \mathrm{~nm})$, indicating that the bulk channels, if present, are 2D with regard to WAL and will be contributing to the measured WAL signal when $E_{f}$ is located in the bulk band as pointed out previously for magnetotransport data of $\mathrm{Bi}_{2} \mathrm{Te}_{3}$ samples $[41,46]$. 
Page 92 Chapter 5. Gate-tunable transport properties of in-situ capped $\mathrm{Bi}_{2} \mathrm{Te}_{3}$ topological ...

Schematic band diagrams are depicted in Fig. 5.3(c) illustrating the evolution of the shift of the Fermi level for the three different back-gate voltage regimes. Our data suggest that at low temperature, we can use the back-gate to tune the Fermi level all the way from the $B C B$ to the topological surface states; and then to the BVB. In most cases, WAL data in TI materials are governed by the competition between phase coherence time $\tau_{\varphi}$ and the surface-to-bulk scattering time $\tau_{S B}[32,44,47,48,49]$. If substantial surface-to-bulk scattering is present, the effective phase coherence time will be much larger than the surface-to-bulk scattering time, $\tau_{\varphi} \gg \tau_{S B}$, resulting in a single effective $2 \mathrm{D}$ transport channel with a prefactor of -0.5 to WAL. In the opposite limit, $\tau_{\varphi} \ll \tau_{S B}$, surface states and bulk are decoupled. The latter scenario is much more likely in regime II in our samples, once the bulk carriers have been depleted, see Figure 5.3. Each surface constitutes a separate coherent channel of $\alpha=-0.5$, leading to a correction to the sample conduction of the order of $\alpha=-1$. Whereas the topological surface states contribute with a negative sign, a positive correction with $\alpha \approx+1$ is expected from bulk conduction channels with weak spin-orbit coupling (giving rise to weak localization), such as expected for the Fermi level being close to the bottom of the band [42]. The convolved nature of the decoupling of bulk and surface contributions [50] prevents a direct summation of the different channels, but the trend away from $\alpha=-1$ towards higher values due to the presence of the bulk conduction band can be clearly understood.

After depleting the bulk carriers, region II (Fig. 5.3(c), center), the Fermi level $E_{\mathrm{f}}$ crosses only the topological surface states. The two surfaces are decoupled, and we expect WAL with $\alpha=-1$, which is consistent with the observed value. Similar bottom-gating WAL results with $\alpha$ tuned from -0.5 to -1 has been reported in $\mathrm{Bi}_{2} \mathrm{Se}_{3}$ thin films grown on STO $[111][27,28]$ and $\mathrm{Bi}_{2} \mathrm{Te}_{3}$ nanoribbons placed on a STO [100] substrate [30]. It should be pointed out that this scenario demands that both conduction channels have nearly identical dephasing fields, which requires good control of the Fermi level and surface morphology $[28,29]$. At negative $V_{b g}, \alpha$ saturates at a value close to unity $(\alpha \sim-1.1)$ indicating that the surfaces stay decoupled as we lower the Fermi level (region III in Fig. 5.3(c) left-hand side).

\subsubsection{Back-gate dependence of the in-situ Te-capped $\mathrm{Bi}_{2} \mathrm{Te}_{3}$ film}

In Fig. 5.4(a), we plot the carrier density $n_{2 \mathrm{D}}$ and the sheet resistance of the Te-capped sample. The sheet carrier density values were extracted from the linear part of the low-field Hall resistance $R_{\mathrm{xy}}(B)$. The prefactor $\alpha$ and the dephasing length $l_{\varphi}$ obtained from fit to equation 5.1 are shown in Fig. 5.4(b) for different back-gate voltages. We observe a similar characteristic as in the $\mathrm{Al}_{2} \mathrm{O}_{3}$-capped $\mathrm{Bi}_{2} \mathrm{Te}_{3}$ film. The change in the total number of (mobile) charge carriers is 
(a)

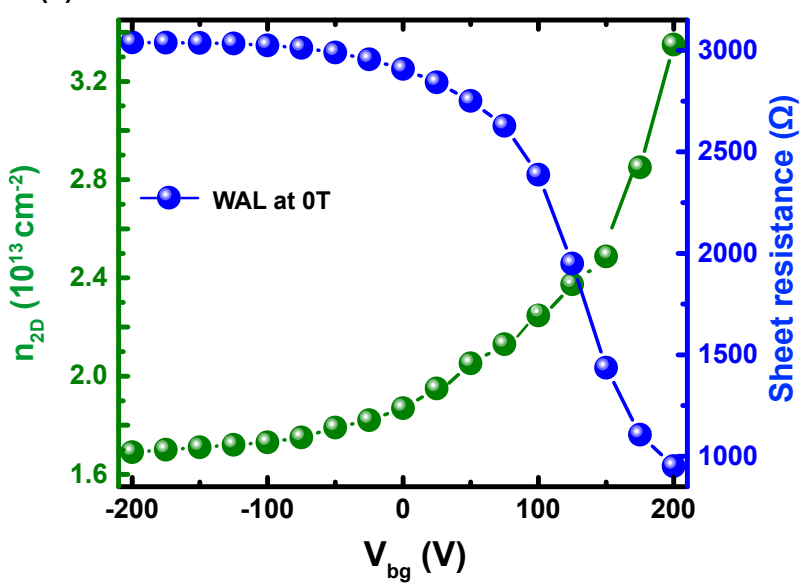

(b)

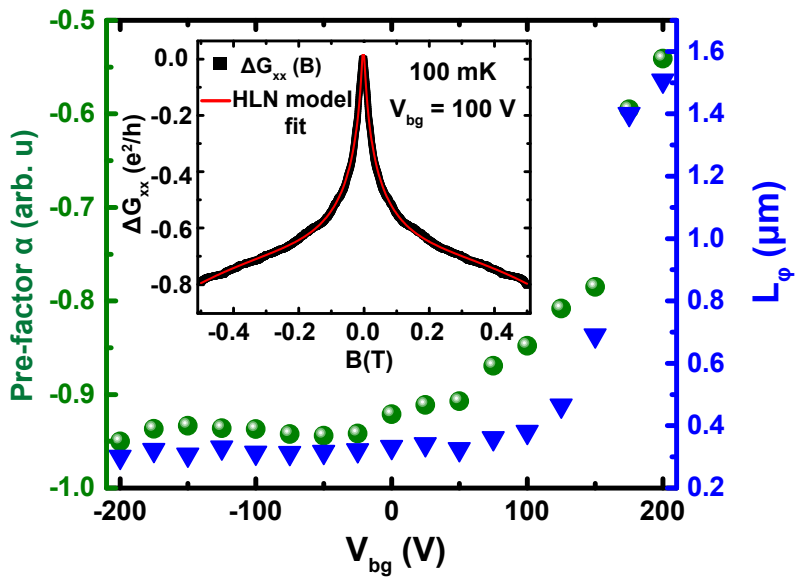

Figure 5.4: Transport characteristics of a $\mathrm{Bi}_{2} \mathrm{Te}_{3}$ film capped in-situ with a $6 \mathrm{~nm}$ Te layer. (a) Back-gate voltage $\left(V_{b g}\right)$ dependence of the $2 \mathrm{D}$ carrier density extracted from the zero-field slope of $R_{\mathrm{xy}}(B)$ and of the sheet resistance. (b) Back-gate dependence of the WAL effect of an in-situ Te-capped $\mathrm{Bi}_{2} \mathrm{Te}_{3}$ film. The prefactor $\alpha$ and the dephasing length $l_{\varphi}$ as function of $V_{b g}$, extracted from best fits to equation (5.1) as illustrated in the inset.

$\Delta n_{2 \mathrm{D}}=1.5 \times 10^{13} \mathrm{~cm}^{-2}$ (see Fig. 5.4(a)). This variation in charge carriers is comparable to the values reported recently for bottom-gated $\mathrm{Se}$-capped $\mathrm{Bi}_{2} \mathrm{Se}_{3}$ films grown on an amorphous $\mathrm{SiO}_{2} / \mathrm{Si}$ substrate [51]. Sweeping the back-gate, both the sheet resistance and the carrier concentration saturate at around $-50 \mathrm{~V}$, the lowest carrier density being $n_{2 \mathrm{D}} \simeq 1.7 \times 10^{13} \mathrm{~cm}^{-2}$ at $-200 \mathrm{~V}$. Although we do not observe a maximum in the sheet resistance or the ambipolar effect in the $R_{\mathrm{xy}}(B)$ data within the gate voltage range, the sharp increase in the sheet resistance together with the corresponding sharp drop in 2D carrier density suggest that we move the Fermi level through the bulk band gap region of the $\mathrm{Bi}_{2} \mathrm{Te}_{3}$ band structure diagram. At these energies the transport properties are dominated by the surface states as the bulk conductivity is significantly reduced $[51,52,53,47]$.

Fits of the magnetoconductance curves to equation (5.1) (see inset Fig. 5.4(b)) were carried out for all gate voltages. We observe a distinct modulation of the prefactor $\alpha$ and the dephasing length $l_{\varphi}$ with $V_{b g}$ shown in Fig. 5.4(b). The results agree qualitatively with the $\mathrm{Al}_{2} \mathrm{O}_{3}$-capped $\mathrm{Bi}_{2} \mathrm{Te}_{3}$ sample in that both, $\alpha$ and $l_{\varphi}$, decrease as $V_{b g}$ is reduced. Again, $\alpha$ approaches $-1 / 2$ for $V_{b g}>150 \mathrm{~V}$ suggesting a single conduction channel coupled by a conducting bulk or, alternatively, a weak localization correction of $\sim 0.5 e^{2} / h$ to the conductance from a decoupled bulk conduction band [42]. As the bulk carriers are depleted, $\alpha \approx-1$, suggesting that each surface acts as an independent coherent transport channel with comparable dephasing fields. 
Page 94 Chapter 5. Gate-tunable transport properties of in-situ capped $\mathrm{Bi}_{2} \mathrm{Te}_{3}$ topological ...

\begin{tabular}{|l|l|l|l|}
\hline Sample/Substrate & Capped or Uncapped & $\mathbf{n}_{\mathbf{2 D}}\left(\mathbf{c m}^{-\mathbf{2}}\right)$ & $\begin{array}{l}\mu\left(\mathbf{c m}^{2} / \mathbf{V . s}\right) \\
\mathbf{a t} \mathbf{B}=\mathbf{0 ~ T}\end{array}$ \\
\hline Sample-I/ STO [111] & Uncapped & $4.3 \times 10^{13}$ & 964 \\
\hline Sample-II/ STO [111] & Uncapped & $6.2 \times 10^{13}$ & 915 \\
\hline Sample-III/ $\mathrm{Al}_{2} \mathrm{O}_{3}[0001]$ & Capped with $\mathrm{Al}_{2} \mathrm{O}_{3}$ & $9.5 \times 10^{12}$ & 1206 \\
\hline Sample-IV/ STO [111] & Capped with $\mathrm{Al}_{2} \mathrm{O}_{3}$ & $3.8 \times 10^{12}$ & 1594 \\
\hline Sample-V/ STO [111] & Uncapped & $3.8 \times 10^{13}$ & 826 \\
\hline Sample-VI/ STO [111] & Uncapped & $2.8 \times 10^{13}$ & 1023 \\
\hline Sample-VII/ STO [111] & Uncapped & $1.2 \times 10^{14}$ & 41 \\
\hline Sample-VIII/ STO [111] & Capped with Te & $1.8 \times 10^{13}$ & 612 \\
\hline
\end{tabular}

Table 5.1: Comparison of the charge carrier densities and the mobilities at zero gate voltage. All capped and uncapped thin films are of the same thickness $(15 \mathrm{~nm})$, and they were grown under the same conditions. Samples IV and VIII refer to the $\mathrm{Al}_{2} \mathrm{O}_{3}$ - and Te-capped samples, respectively, which are discussed in detail in the main text.

To asses the role of the capping layers in protecting the topological surface states of $\mathrm{Bi}_{2} \mathrm{Te}_{3}$ thin films, we compare transport characteristics from capped films to uncapped ones. We choose films of the same thickness $(15 \mathrm{~nm})$, which were grown under the same conditions. Table 5.1 summarizes the extracted low field carrier densities and the corresponding zero magnetic field mobilities for the two capped samples (sample IV and VIII), we presented in this work, and of a capped $\mathrm{Bi}_{2} \mathrm{Te}_{3}$ film grown on sapphire (sample-III) together with five other uncapped $\mathrm{Bi}_{2} \mathrm{Te}_{3}$ films grown on STO. Clearly, we observe an improvement in the transport characteristics (i.e., lower intrinsic carrier densities while maintaining similar or high mobilities) for the in-situ $\mathrm{Al}_{2} \mathrm{O}_{3^{-}}$ and Te-capped films.

\subsection{Conclusion and outlook}

We demonstrated the effectiveness of in-situ capping for preserving topological surface states in bulk insulating $\mathrm{Bi}_{2} \mathrm{Te}_{3}$ thin films. For this purpose, we tested capping with $\mathrm{Al}_{2} \mathrm{O}_{3}$ and $\mathrm{Te}$ layers and found that both methods lead to a significant decrease in the measured carrier density by protecting the TI surfaces from adsorbates and other contaminations. We back-gated the samples using the $\mathrm{SrTiO}_{3}$ substrate as a dielectric, and we found that we were able to vary the carrier densities by $\sim 1.5-2 \times 10^{13} \mathrm{~cm}^{-2}$ carriers in the gate voltage range of $\pm 200 \mathrm{~V}$. In samples with low intrinsic doping, this modulation is sufficient to move from a bulk-dominated transport regime to a 
bulk insulating sample with decoupled surface states, which is evidenced by a doubling of the weak antilocalization correction to the sample conductance. The ability to access TI surface states is an important step toward device applications in the field of quantum information and spintronics. We showed that thin film deposition techniques of $\mathrm{TI}$ materials with low intrinsic doping paired with protective capping of the topological surface and effective depletion of bulk carriers by back-gating provide a technological platform for large-scale integration of such electronic devices. 


\section{Bibliography}

[1] L. Fu, C. L. Kane, and E. J. Mele, Phys. Rev. Lett. 98, 106803 (2007).

[2] C. L. Kane and E. J. Mele, Phys. Rev. Lett. 95, 146802 (2005).

[3] H. Zhang, C. -X. Liu, X. -L Qi, X. Dai, Z. Fang, S. -C. Zhang, Nature Phys. 5, 438 (2009).

[4] J. E. Moore, Nature 464, 194 (2010).

[5] D. Hsieh, Y. Xia, D. Qian, L. Wray, J. H. Dil, F. Meier, J. Osterwalder, L. Patthey, J. G. Checkelsky, N. P. Ong, A. V. Fedorov, H. Lin, A. Bansil, D. Grauer, Y. S. Hor, R. J. Cava and M. Z. Hasan, Nature 460, 1101 (2009).

[6] C. Jozwiak, C. -H. Park, K. Gotlieb, C. Hwang, D. -H. Lee, S. G. Louie, J. D. Denlinger, C. R. Rotundu, R. J. Birgeneau, Z. Hussain and A. Lanzara, Nature Phys. 9, 293 (2013).

[7] Y. L. Chen, J. G. Analytis, J. -H. Chu, Z. K. Liu, S. -K. Mo, X. L. Qi, H. J. Zhang, D. H. Lu, X. Dai, Z. Fang, S. C. Zhang, I. R. Fisher, Z. Hussain and Z . -X. Shen, Science 325, 5937 (2009).

[8] Z. Alpichshev, J. G. Analytis, J. -H. Chu, I. R. Fisher, Y. L. Chen, Z. X. Shen, A. Fang, and A. Kapitulnik, Phys. Rev. Lett. 104, 016401 (2010).

[9] P. Ngabonziza, R. Heimbuch, N. de Jong, R. A. Klaassen, M. P. Stehno, M. Snelder, A. Solmaz, S. V. Ramankutty, E. Frantzeskakis, E. van Heumen, G. Koster, M. S. Golden, H. J. W. Zandvliet, and A. Brinkman, Phys. Rev. B 92, 035405 (2015).

[10] K. Hoefer, C. Becker, D. Rata, J. Swanson, P. Thalmeier, and L. H. Tjeng, Proc. Natl. Acad. Sci. USA 111, 14979 (2014).

[11] K. Hoefer, C. Becker, S. Wirth, and L. H. Tjeng, AIP Adv. 5, 097139 (2015). 
[12] S. E. Harrison, B. Zhou, Y. Huo, A. Pushp, A. J. Kellock, S. S. P. Parkin, J. S. Harris, Y. Chen, and T. Hesjedal, Appl. Phys. Lett. 105, 121608 (2014).

[13] K. Virwani, S. E. Harrison, A. Pushp, T. Topuria, E. Delenia, P. Rice, A. Kellock, L. CollinsMclntyre, J. Harris, T. Hesjedal, and S. Parkin, Appl. Phys. Lett. 105, 241605 (2014).

[14] S. E. Harrison, S. Li, Y. Huo, B. Zhou, Y. L. Chen, and J. S. Harris, Appl. Phys. Lett. 102, 171906 (2013).

[15] T. Chen, Q. Chen, K. Schouteden, W. Huang, X. Wang, Z. Li, F. Miao, X. Wang, Z. Li, B. Zhao, S. Li, F. Song, J. Wang, B. Wang, C. Van Haesendonck and G. Wang, Nat. Commun. 75, 5022 (2014).

[16] M. Brahlek, N. Koirala, M. Salehi, N. Bansal, and S. Oh, Phys. Rev. Lett. 113, 026801 (2014).

[17] X. Yu, L. He, M. Lang,W. Jiang, F. Xiu, Z. Liao, Y. Wang, X. Kou, P. Zhang, J. Tang, G. Huang, J. Zou and K. L. Wang, Nanotechnology 24, 015705 (2013).

[18] H. Liu and P. D. Ye, Appl. Phys. Lett. 99, 052108 (2011).

[19] F. Yang, A. A. Taskin, S. Sasaki, K. Segawa, Y. Ohno, K. Matsumoto, and Y. Ando, Appl. Phys. Lett. 104, 161614 (2014).

[20] J. Wiedenmann, E. Bocquillon, R.S. Deacon S. Hartinger1, O. Herrmann, T.M. Klapwijk, L. Maier, C. Ames1, C. Brüne, C. Gould, A. Oiwa, K. Ishibashi, S. Tarucha, H. Buhmann and L.W. Molenkamp, Nat. Commun. 7, 10303 (2016).

[21] C. Kurter, A. D. K. Finck, P. Ghaemi, Y. S. Hor, and D. J. Van Harlingen, Phys. Rev. B 90, 014501 (2014).

[22] X. He, T. Guan, X. Wang, B. Feng, P. Cheng, L. Chen, Y. Li, and K. Wu, Appl. Phys. Lett. 101, 123111 (2012).

[23] H. Yuan, H. Liu, H. Shimotani, H. Guo, M. Chen, Q. Xue, and Y. Iwasa, Nano Lett. 11, 2601 (2011).

[24] J. Xiong, Y. Khoo, S. Jia, R. J. Cava, and N. P. Ong, Phys. Rev. B 88, 035128 (2013).

[25] M. Lang,L. He,F. Xiu, X. Yu, J. Tang, Y. Wang, X. Kou,W. Jiang, A. V. Fedorov, and K. L. Wang, Nano Lett. 6, 295 (2012). 
[26] J. Moulder and J. Chastain, Handbook of X-ray Photoelectron Spectroscopy: A Reference Book of Standard Spectra for Identification and Interpretation of XPS Data (Physical Electronics Division, Perkin-Elmer Corporation, 1992).

[27] G. Zhang, H. Qin , J. Chen , X. He , L. Lu , Y. Li and K. Wu, Adv. Funct. Mater. 21, 2351 (2011).

[28] J. Chen, X. Y. He, K. H.Wu, Z. Q. Ji, L. Lu, J. R. Shi, J. H. Smet, and Y. Q. Li, Phys. Rev. B 83, 241304(R) (2011).

[29] J. Chen, H. J. Qin, F. Yang, J. Liu, T. Guan, F.M. Qu, G. H. Zhang, J. R. Shi, X. C. Xie, C. L. Yang, K. H.Wu, Y. Q. Li, and L. Lu, Phys. Rev. Lett. 105, 176602 (2010).

[30] L. A. Jauregui , M. T. Pettes, L. P. Rokhinson, L. Shi and Y. P. Chen, Sci. Rep. 5, 8452 (2015).

[31] F. Xiu, L. He, Y. Wang, L. Cheng, L. -T. Chang, M. Lang, G. Huang, X Kou, Y. Zhou, X. Jiang, Z. Chen, J. Zou, A. Shailos and K. L.Wang, Nat. Nano. 6, 216 (2011).

[32] J. Tian, C. Chang, H. Cao, K. He, X. Ma, Q. Xue, and Y. P. Chen, Sci. Rep. 4, 4859 (2014).

[33] Y. Ando, J. Phys. Soc. Jpn. 82, 102001 (2013).

[34] J. Lee, J. Park, J. Lee, J. Kim, and H. Lee, Phys. Rev. B 86, 245321 (2012).

[35] G. S. Jenkins and D. C. Schmadel, Phys. Rev. B 87, 155126 (2013)

[36] G. S. Jenkins, A. B. Sushkov, D. C. Schmadel, M. -H. Kim, M. Brahlek, N. Bansal, S. Oh, and H. D. Drew, Phys. Rev. B 86, 235133 (2012).

[37] M. Brahlek, N. Koirala, N. Bansal, S. Oh, Solid State Communications 215-216, 54 (2015).

[38] S. Hikami, A. I. Larkin and Y. Nagaoka, Prog. Theor. Phys. 63, 707 (1980).

[39] H. Z. Lu and S. Q. Shen, Phys. Rev. B 84, 125138 (2011).

[40] I. Garate and L. Glazman, Phys. Rev. B 86, 035422 (2012).

[41] S. -P. Chiu and J. -J. Lin, Phys. Rev. B 87, 035122 (2013).

[42] H. -Z. Lu and S. -Q. Shen, Phys. Rev. Lett. 112, 146601 (2014).

[43] J. Wang, A. M. DaSilva, C. -Z. Chang, K. He, J. K. Jain, N. Samarth, X. -C. Ma, Q. -K. Xue, and M. H. W. Chan, Phys. Rev. B 83, 245438 (2011). 
[44] H. Steinberg, J. -B. Laloë, V. Fatemi, J. S. Moodera, and P. Jarillo-Herrero, Phys. Rev. B 84, 233101 (2011).

[45] S. Maekawa, and H. Fukuyama, J. of the Physical Society of Japan 50(8), 2516 (1981).

[46] H. -T. He, G. Wang, T. Zhang, I. -K. Sou, G. K. L. Wong, and J. -N. Wang, Phys. Rev. Lett. 106, 166805 (2011).

[47] D. Kim, P. Syers, N. P. Butch, J. Paglione, and M. S. Fuhrer, Nat. Commun. 4, 2040 (2013).

[48] M. Lang, L. He, X. Kou, P. Upadhyaya, Y. Fan, H. Chu, Y. Jiang, J. H. Bardarson, W. Jiang, E. S. Choi, Y. Wang, N. C. Yeh, J. Moore, and K. L. Wang, Nano Lett. 13, 48 (2013).

[49] M. Brahlek, N. Koirala, M. Salehi, N. Bansal, and S. Oh, Phys. Rev. Lett. 113, 026801 (2014).

[50] Z. Li, I. Garate, J. Pan, X. Wan, T. Chen, W. Ning, X. Zhang, F. Song, Y. Meng, X. Hong, X. Wang, L. Pi, X. Wang, B. Wang, S. Li, M. A. Reed, L. Glazman and G. Wang, Phys. Rev. B 91, 041401(R) (2015).

[51] Y. H. Liu, C.W. Chong, J. L. Jheng, S. Y. Huang, J. C. A. Huang, Z. Li, H. Qiu, S. M. Huang and V. V. Marchenkov, Appl. Phys. Lett. 107, 012106 (2015).

[52] S. S. Hong, J. J. Cha, D. Kong, and Y. Cui, Nat. Commun. 3, 757 (2012).

[53] Y. S. Kim, M. Brahlek, N. Bansal, E. Edrey, G. A. Kapilevich, K. lida, M. Tanimura, Y. Horibe, S. W. Cheong, and S. Oh, Phys. Rev. B 84, 073109 (2011). 

6

\section{Combined gate-tunable Josephson supercurrent and normal state transport in $\mathbf{N b}-\mathrm{Bi}_{2} \mathrm{Te}_{3}-\mathrm{Nb}$ hybrid devices}
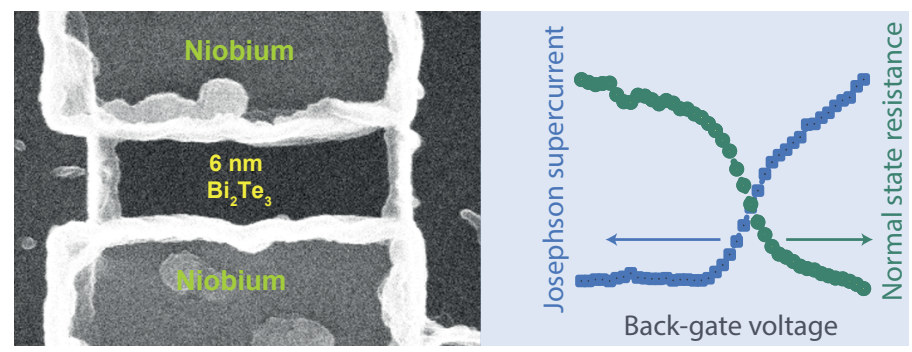

We report on combined gate tunable Josephson junctions and normal state transport in $\mathrm{Bi}_{2} \mathrm{Te}_{3}$ topological insulator thin films grown on $\mathrm{SrTiO}_{3}$ [111]. By using the substrate as a gate dielectric, we tuned the carrier density electrostatically and measured the Josephson supercurrent and the normal state transport properties of our thin film devices. We identify 3 gate voltage ranges with distinct behaviour: A region of intermediate gate bias where the measured quantities change rapidly with the applied electric field, and two saturation regions for large bias of either polarity. The same features are also observed in the carrier density obtained by Hall effect measurements on the same film. We attribute the measured behaviour to band bending and discuss the spatial distribution of supercurrent and its implications for the superconducting proximity effect in thin film samples.

The work presented in this chapter is under preparation as manuscript for publication in a peer-reviewed journal as: M. P. Stehno*,P. Ngabonziza*, H. Myoren, and A. Brinkman, "Critical current distribution in bottomgated topological insulator Josephson junctions".

* Both authors contributed equally to this work. 


\subsection{Introduction}

The field of topological superconductivity has drawn a lot of interest in the condensed matter research community. It offers the unique prospect of combining properties of the band structure topology with superconducting pairing to generate a new low-energy effective mode of the electronic system, a Majorana fermion (MF) $[1,2,3]$. The inherited topological character of such excitations allows for a novel way of encoding quantum information, essential for improved, faulttolerant quantum computation schemes [4, 5]. Recently, signatures of MFs have been found in quantum devices based on various materials such as semiconductor nanowire-superconductor contacts $[6,7,8,9,10,11]$, Josephson junctions with 2-dimensional and 3-dimensional topological insulator $(\mathrm{TI})$ weak links $[12,13]$; and by means of scanning tunneling microscopy in chains of ferromagnetically coupled atoms on superconductor surfaces $[14,15]$. Common to all these experiments is that superconducting correlations are induced by bringing the material in contact with an ordinary BCS-type superconductor. Obtaining a strong superconducting proximity effect and a large effective gap in the quasiparticle spectrum are instrumental for the observation of Majorana physics. Yet, only recently the topic has been approached theoretically $[16,17]$, and little is known about the magnitude and spatial dependence of the proximity effect experimentally. In most available TI materials, a nonuniform charge distribution of intrinsic dopants [18] and extrinsic impurity contaminations $[19,20]$ are present. As a result, band bending [21, 22] occurs close to interfaces. These effects also need to be taken into consideration when the proximity coupling between superconductors and TI materials is discussed.

Since few candidate materials for intrinsic topological superconductivity have been identified so far $\mathrm{Sr}_{2} \mathrm{RuO}_{4}$ [23, 24], UPt 3 [25], and $\mathrm{Cu}_{x} \mathrm{Bi}_{2} \mathrm{Se}_{3}[26,27]$, and their underlying pairing symmetries are subject to ongoing investigations, research has focused on an alternative route to creating topological superconductivity. Early on, Fu and Kane proposed that, a network of Josephson junctions on the surface of a 3-dimensional $\mathrm{TI}$ is a realization of an artificial topological superconductor [1]. A first observation of the Josephson effect was reported in devices fabricated from $\mathrm{Bi}_{2} \mathrm{Se}_{3}$ single crystals with aluminum/titanium electrodes [28]. Subsequently, Josephson junctions were prepared in a variety of material combinations using both, exfoliated $\mathrm{TI}$ crystals and TI thin films $[12,13,29,30,31,32,33,34,35,36,37,38,39,40,41,42,43]$. In most studies, it was found that a large fraction of the supercurrent was carried by ballistic Andreev bound states (ABS), attributed to the topological surface state (TSS). Most recently, the suppression of odd-numbered Shapiro steps, a key signature for the presence of ungapped, $4 \pi$-periodic Andreev bound states, was observed by Wiedenmann, et al., [12] in Josephson devices made with strained $\mathrm{HgTe}$ weak links. It was argued that only a small number of ABS was gapless and that the 
majority of the supercurrent was carried by ordinary ABS.

Ungapped modes emerge only on the topological surface(s) whereas higher energy bands of surface states [44], surface modes with finite perpendicular momentum component [45], and ordinary bulk modes are always gapped. (The distinction is made since a topological surface with ungapped $A B S$ may be generated inside the bulk of Tls which are highly doped on the surface [42]). The participation of ordinary, $2 \pi$-periodic modes is dictated by the (thermal) occupation of the respective levels in the $A B S$ spectrum and the transition rate between the ungapped states and higher energy bands. It can be reduced by engineering the effective coupling between $\mathrm{TI}$ and superconductor, or, vice versa, the induced gap. The bulk plays an important role in the optimization as thinner samples minimize the contribution of bulk modes by decreasing the total number of dopant charges. This also affects the electrostatic screening of charged surface contaminations and applied gate fields. When the sample thickness approaches the length scale of electrostatic screening or becomes comparable to the typical spreading of the TSS wave function into the bulk, the coupling between the superconductor and the TI material may change substantially. To address such effects, we studied the supercurrent in Josephson devices with weak links made from thin $\mathrm{Bi}_{2} \mathrm{Te}_{3}$ films with low intrinsic dopant density. By using the substrate as a gate dielectric, we are able to change the carrier density in the bottom portion of the TI film electrostatically. The gate voltage dependence of the critical current shows distinct differences for the two electrical field polarities, a feature that is also observed in the carrier density obtained by Hall effect measurements on the same film. We attribute this behavior to band bending and discuss the spatial distribution of supercurrent and its implications for the superconducting proximity effect in thin film samples.

\subsection{Thin films quality and Josephson junctions characteristics}

Recent improvements in the molecular-beam epitaxy of topological insulator materials allow us to obtain high quality thin films of $\mathrm{Bi}_{2} \mathrm{Te}_{3}$ with very low intrinsic doping $[19,46]$. The as-grown material features a Fermi level in the bulk band gap (see Fig. 6.1) which is achieved without resorting to techniques such as counterdoping [47, 48], p-n layer growth [49, 50, 51], or using (off-)stoichiometric ternary [52] and quarternary [53,54] compounds. A subsequent, moderate increase in the doping level of these films is found to originate from ex-situ contamination of the film surface during sample transfer and structuring $[19,20,46]$. 
Page 104 Chapter 6. Combined gate-tunable Josephson supercurrent and normal state ...

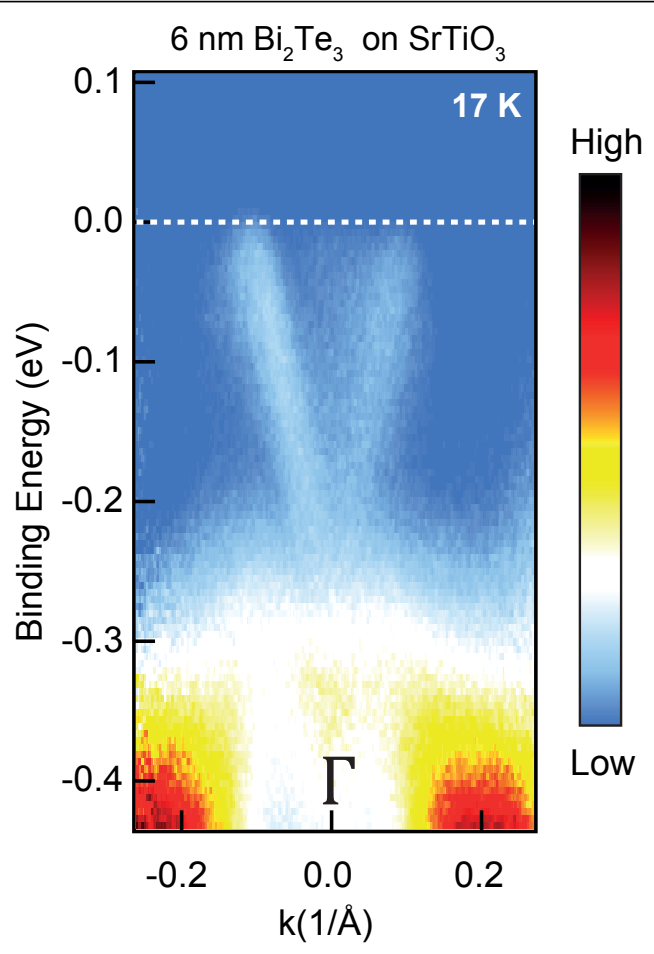

Figure 6.1: Thin film quality. An angle resolved photoemission spectroscopy (ARPES) spectra around the $\Gamma$ point of the $6 \mathrm{~nm} \mathrm{Bi} \mathrm{Te}_{3}$ thin film. Josephson junctions discussed below were fabricated on this sample. The white dashed line indicates the position of the Fermi level, which is in the bulk band gap without bulk band contribution. Details on ARPES measurements on similar samples are given in section 4.3 of chapter 4 .

This is explained by our choice of material, $\mathrm{Bi}_{2} \mathrm{Te}_{3}$, which is less prone to forming surface vacancies compared to, e.g., $\mathrm{Bi}_{2} \mathrm{Se}_{3}[18,55,56,57]$.

To study the proximity effect in the bulk and the contribution of the bottom surface to the supercurrent, we compare devices prepared from $\mathrm{Bi}_{2} \mathrm{Te}_{3}$ thin films of average thickness $6 \mathrm{~nm}$ and $15 \mathrm{~nm}$ [i.e., 6 quintuple layers (QL) and $15 \mathrm{QL}$ of $\mathrm{Bi}_{2} \mathrm{Te}_{3}$ material], respectively. The choice of $6 \mathrm{~nm}$ for the thinner film is motivated by the objective of eliminating most of the TI bulk without opening a large hybridization gap $[58,59]$. The value for the thicker film is chosen to be comparable to the electrostatic screening length of $\gtrsim 10 \mathrm{~nm}$ in uncapped films with typical carrier densities of $\sim 10^{13} \mathrm{~cm}^{-2}[18,60]$. The films were deposited on $\mathrm{SrTiO}_{3}$ [111] single crystalline substrates (STO) in a two-step MBE process [19]. Subsequently, Hall bar devices and Josephson junctions ( $\mathrm{JJs}$ ) were patterned using standard electron-beam lithography and sputter deposition of $60 \mathrm{~nm}$ thick $\mathrm{Nb}$ electrodes with a $5 \mathrm{~nm}$ Pd capping layer after cleaning the contact area with a low-power Ar plasma. The device boundaries were shaped by dry etching. The samples were mounted on a printed-circuit board using silver paint to ensure electrical contact between the crystalline substrate and the backgate electrode. The transport characterization was carried out in a dilution refrigerator with heavily filtered signal lines.

A scanning-electron micrograph of a typical $\mathrm{Nb} / \mathrm{Bi}_{2} \mathrm{Te}_{3} / \mathrm{Nb}$ Josephson junction device is shown in Fig. 6.2(a). To define the weak link laterally, we removed the $\mathrm{Bi}_{2} \mathrm{Te}_{3}$ film surrounding the junction area by dry etching, thus eliminating any spurious shunt conductance of $\mathrm{TI}$ material that is not 
(a)

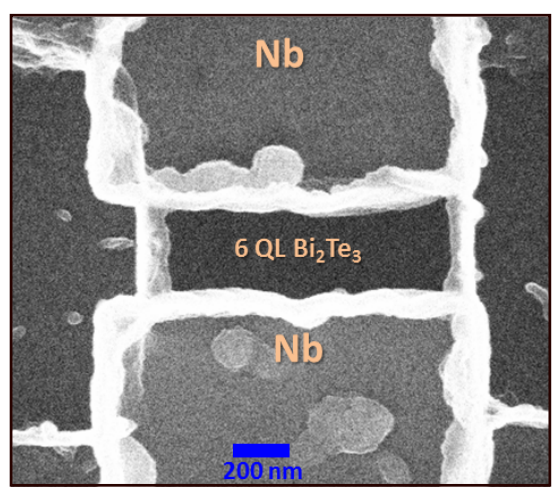

(c)

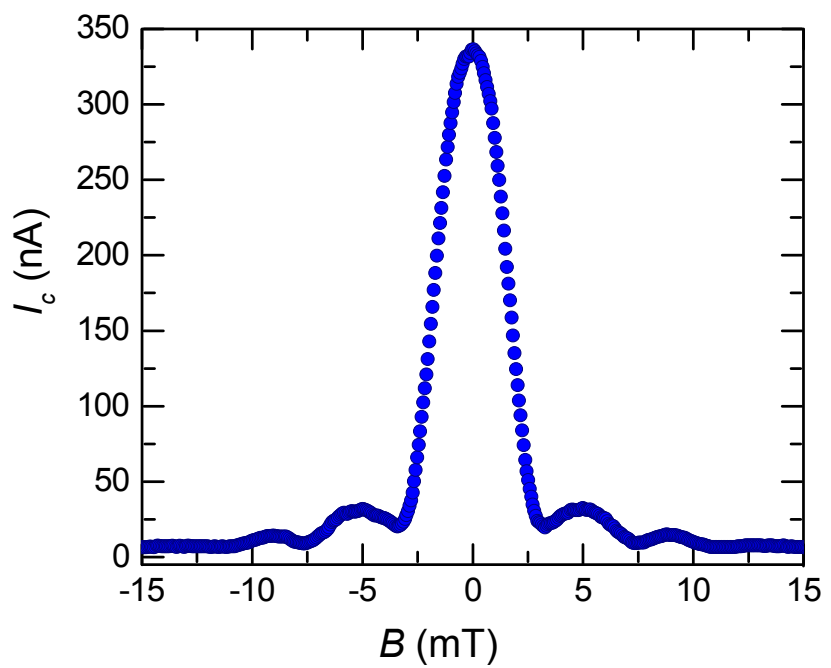

(b)

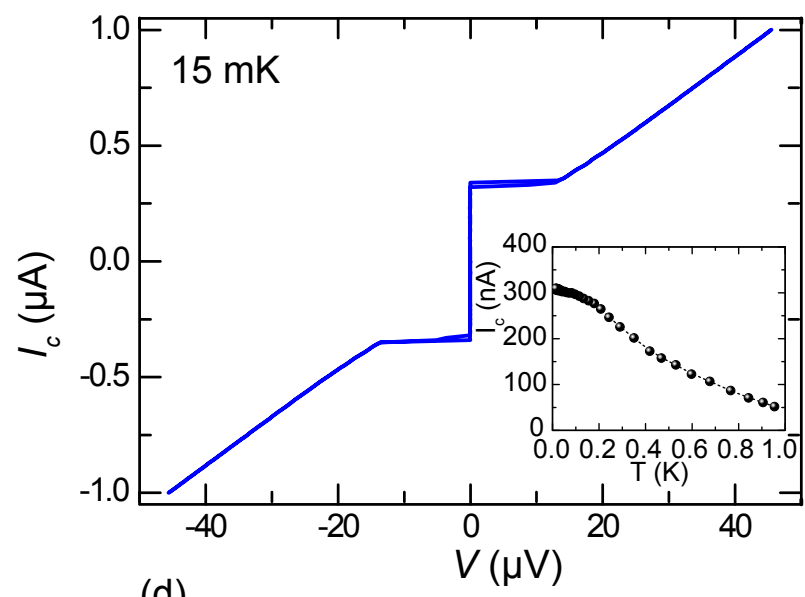

(d)

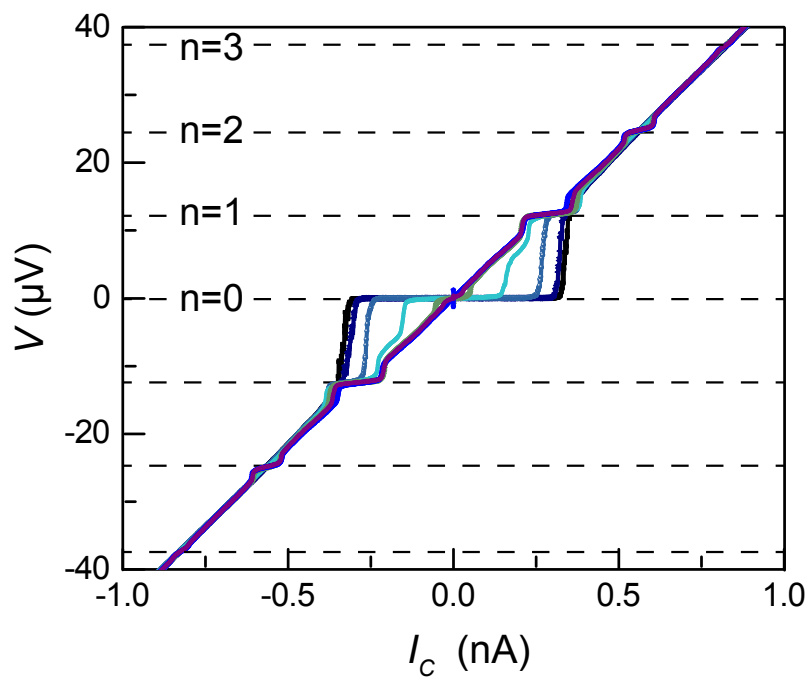

Figure 6.2: $\mathrm{Nb} / \mathrm{Bi}_{2} \mathrm{Te}_{3} / \mathrm{Nb}$ thin film Josephson junctions. A SEM image of a Josephson junction device (a). The width of the weak link is defined by dry etching. The device dimensions are visible clearly by residues of cross-linked photoresist from the etch mask. The I-V characteristic of a Josephson device with $6 \mathrm{QL}$ thick $\mathrm{Bi}_{2} \mathrm{Te}_{3}$ weak link (b). Inset: The temperature dependence of the critical current. Most junctions show a Fraunhofer-like Josephson diffraction pattern (c). Voltage plateaux appear in the $\mathrm{V}$-I curves under microwave irradiation with frequency $6.02 \mathrm{GHz}$ (d). The voltages $V_{n} \simeq n \times(12.4 \mu \mathrm{V})$ for Shapiro steps of order $n$ are indicated.

involved in supercurrent transport. Fig. 6.2(b) shows the current-voltage (I-V) characteristic of a $6 \mathrm{QL}$ Josephson junction device with a weak link width of $860 \mathrm{~nm}$ and length of $250 \mathrm{~nm}$. Before applying gate bias $\left(V_{\mathrm{bg}}=0 \mathrm{~V}\right)$, a maximum Josephson critical current of $342 \mathrm{nA}$ was observed. The temperature dependence of the critical current is plotted in the inset of Fig. 6.2(b). Here, the critical current is defined by the voltage criterion $V \leq 500 \mathrm{nV}$. The device displays a Fraunhofer- 
like Josephson diffraction pattern [see Fig. 6.2(c)]. When irradiated with microwaves of frequency $f=6.02 \mathrm{GHz}$, the I-V curves developed voltage plateaux [61] corresponding to multiples $n$ of the driving frequency $f, V_{n}=\frac{n h f}{2 e} \simeq n \times(12.4 \mu \mathrm{V})$, where $h$ and $e$ are Planck's constant and the elementary charge, respectively. This Shapiro step pattern is shown in Fig. 6.2(d) for several RF drive amplitudes. Both, even and odd $n$ steps are present in our Josephson devices at this frequency.

\subsection{Gate dependence of Josephson supercurrent and normal state transport}

We are able to change the carrier density in the $\mathrm{TI}$ electrostatically by applying a voltage between a device and the counterelectrode on the backside of the STO substrate. The electric field redistributes charges which results in filling (or emptying) of defect sites [i.e., charge traps] on the surface(s) and in the bulk of the material. When we plot a measured quantity against the applied backgate voltage, we observe an initial shift toward higher gate voltages. After (several) gate sweep(s), the hysteretic curves become reproducible. Therefore, measurements are carried out by starting at the maximum (positive) gate voltage and reducing the gate bias subsequently.

After priming the sample by sweeping the backgate voltage over the full bias range multiple times, we measured the I-V characteristics at $15 \mathrm{mK}$, the base temperature of our dilution refrigerator. In the following, the Josephson critical current is defined by the maximum applied current that fullfils a voltage criterion $V \leq 1 \mu \mathrm{V}$. An estimate of the normal state resistance of the device is obtained by a linear fit to the I-V curve at large current bias $(>1.5 \mu \mathrm{A})$. To characterize the effect of the backgate on the $\mathrm{Nb} / \mathrm{Bi}_{2} \mathrm{Te}_{3} / \mathrm{Nb}$ Josephson junctions, we plot the critical current $\left(I_{\mathrm{c}}\right)$ and the normal state resistance $\left(R_{N}\right)$ as a function of backgate voltage $\left(V_{\mathrm{bg}}\right)$ in Fig. 6.3. We present data of two representative devices with similar characteristic energy of the Josephson coupling $\left(I_{\mathrm{c}} R_{N} \approx 18 \mu \mathrm{V}\right)$ at large positive backgate voltage $\left(V_{\mathrm{bg}}=200 \mathrm{~V}\right)$. In Fig. 6.3(a), we display $I_{\mathrm{c}}$ and $R_{N}$ curves of a $250 \mathrm{~nm}$ long and $860 \mathrm{~nm}$ wide Josephson junction that is fabricated on a $6 \mathrm{~nm}$ thick $\mathrm{Bi}_{2} \mathrm{Te}_{3}$ film (JJ1). The data in Fig. 6.3(b) are obtained from a $80 \mathrm{~nm}$ long and $200 \mathrm{~nm}$ wide device with a $\mathrm{Bi}_{2} \mathrm{Te}_{3}$ weak link of $15 \mathrm{~nm}$ thickness (JJ2). Over the entire gate bias range $( \pm 200 \mathrm{~V})$, the normal state resistance $R_{N}$ increases by a factor $\gtrsim 2.2$, which is comparable to the change in sheet resistance of the $\mathrm{Bi}_{2} \mathrm{Te}_{3}$ thin films measured in the Hall bar devices. The region of steepest increase is about $100 \mathrm{~V}$ wide and centered around $V_{\mathrm{bg}} \approx 20 \mathrm{~V}$. Starting from $I_{\mathrm{c}}=437 \mathrm{nA}$ at $200 \mathrm{~V}$ backgate bias, the critical current of $\mathrm{JJ} 1$ drops linearly with gate voltage but then decreases more rapidly in the region where the resistance increases strongly. For negative 
Section 6.3. Gate dependence of Josephson supercurrent and normal state transport Page 107
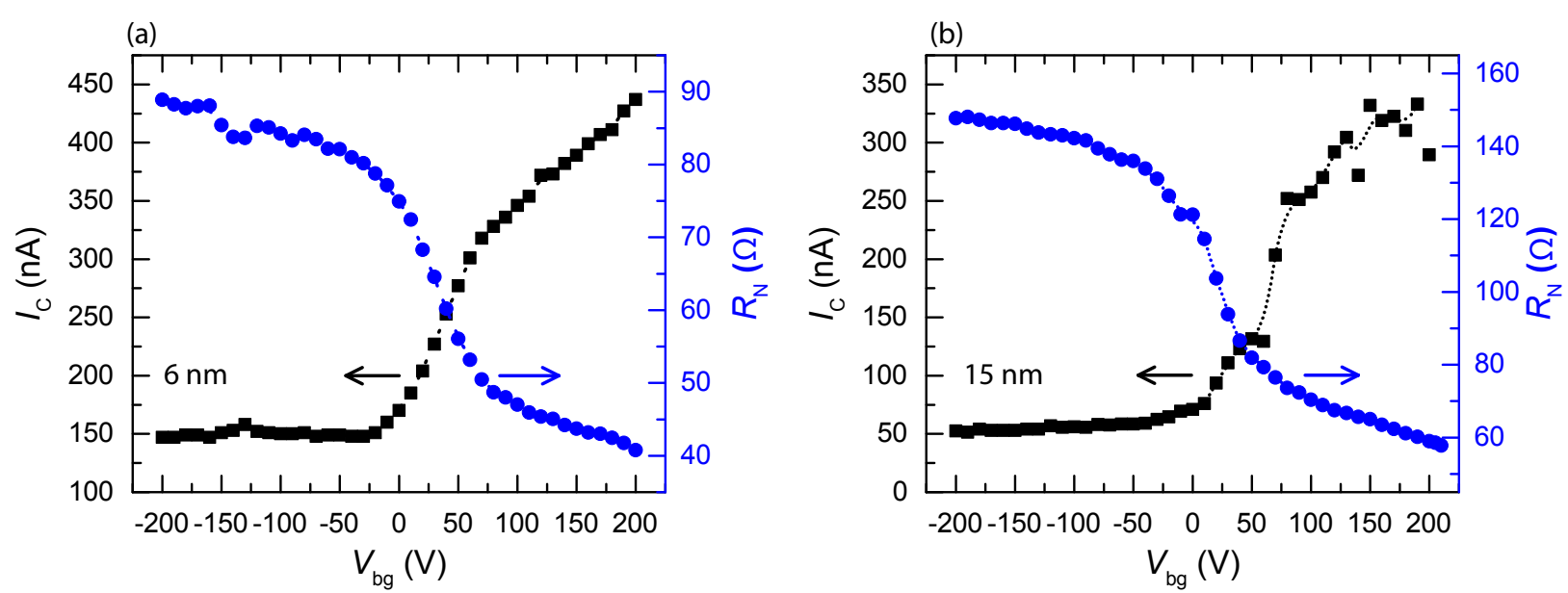

Figure 6.3: Backgate voltage dependence of the normal state resistance and the Josephson critical current for $\mathrm{Nb} / \mathrm{Bi}_{2} \mathrm{Te}_{3} / \mathrm{Nb}$ junctions of (a) $6 \mathrm{~nm}$, and (b) $15 \mathrm{~nm}$ TI film thickness. Whereas the critical current is enhanced for positive backgate voltages, it flattens out when negative gate bias is applied.

gate bias, it flattens out at $147 \mathrm{nA}$. The $I_{\mathrm{c}}-V_{\mathrm{bg}}$ curve of $\mathrm{JJ} 2$ has similar features. However, the critical current exhibits switching noise for $V_{\mathrm{bg}}>70 \mathrm{~V}$.

To explore the role of the charge carrier density and the distribution of carriers in the $\mathrm{TI}$, we measured the magnetotransport properties of the $15 \mathrm{~nm}$ thick $\mathrm{Bi}_{2} \mathrm{Te}_{3}$ film for a sample temperature of $50 \mathrm{mK}$. The measurements were performed in Hall bar geometry using standard lock-in techniques. A small excitation current of $5 \mathrm{nA}$ was chosen to minimize sample heating. The sheet resistance shows a weak antilocalization (WAL) feature at low magnetic fields $(B \lesssim 200 \mathrm{mT})$. After (anti-)symmetrization and inversion of the resistivity matrix, we subtract the conductance at zero field to obtain the conductance difference $\Delta G(B)$, plotted in Fig. 6.4(a). As the backgate voltage is decreased the magnitude of the WAL signal increases. A quadratic background becomes noticeable. To quantify the WAL correction, we assume a function $G_{b}=G_{0}+c_{1} B+c_{2} B^{2}$ for the background and fit the sheet conductance $G$ with the Hikami-Larkin-Nagaoka (HLN) formula $[62,63]$ :

$$
G-G_{b}=\alpha \frac{e^{2}}{2 \pi \hbar}\left[\Psi\left(\frac{1}{2}+\frac{\hbar}{4 e l_{\phi}^{2} B}\right)-\ln \left(\frac{\hbar}{4 e l_{\phi}^{2} B}\right)\right] .
$$

Here, $\Psi$ denotes the digamma function; $e$, and $\hbar$ are the elementary charge, and the reduced Planck's constant, respectively. In Fig. 6.4(c), we plot the prefactor $\alpha$ and the dephasing length $l_{\phi}$. At large positive gate bias, we find $\alpha \sim-1.2$. It drops to $\sim-1.7$ at large negative gate voltage. The transition occurs gradually in the same bias region where we had observed a strong decrease 
Page 108 Chapter 6. Combined gate-tunable Josephson supercurrent and normal state ...

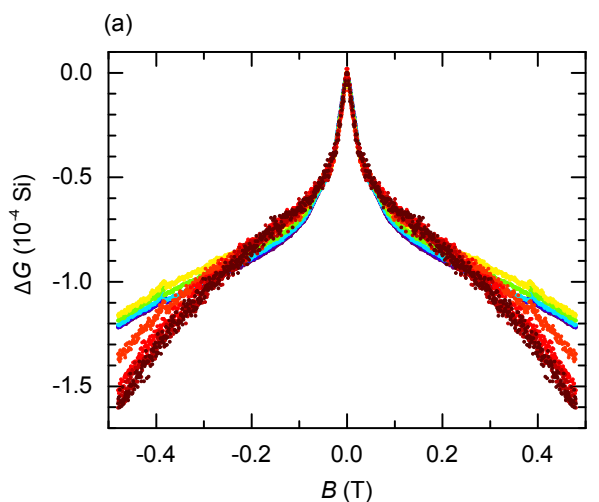

(c)

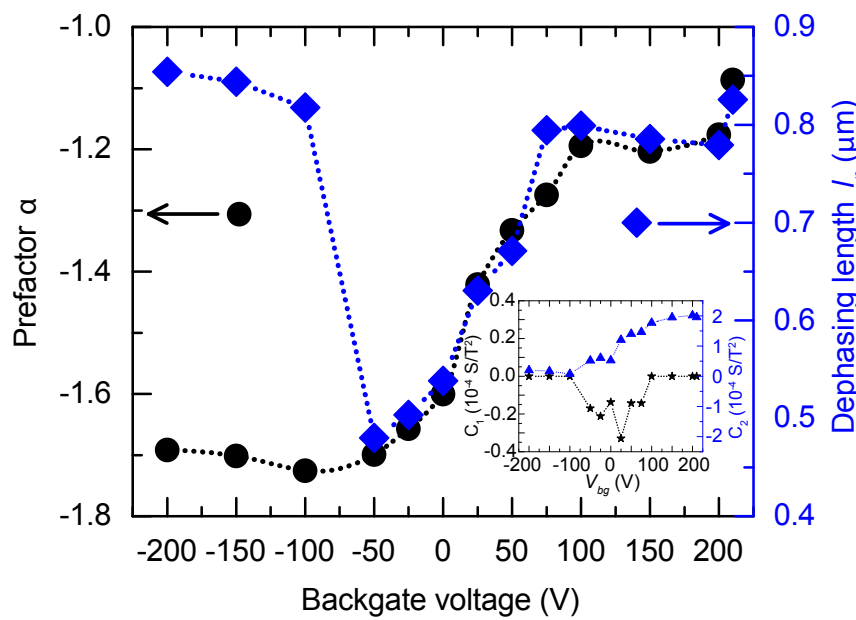

(b)

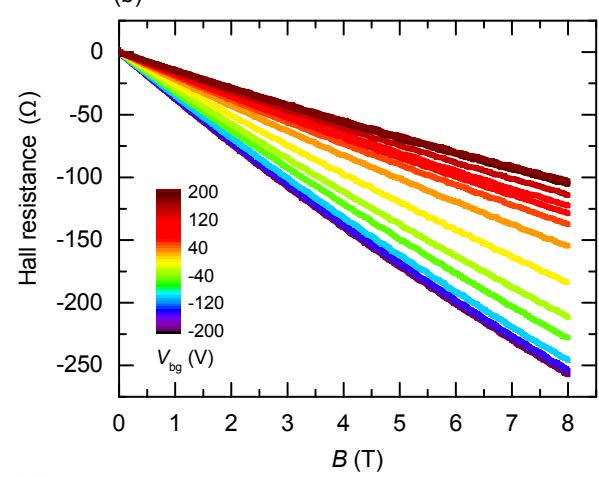

(d)

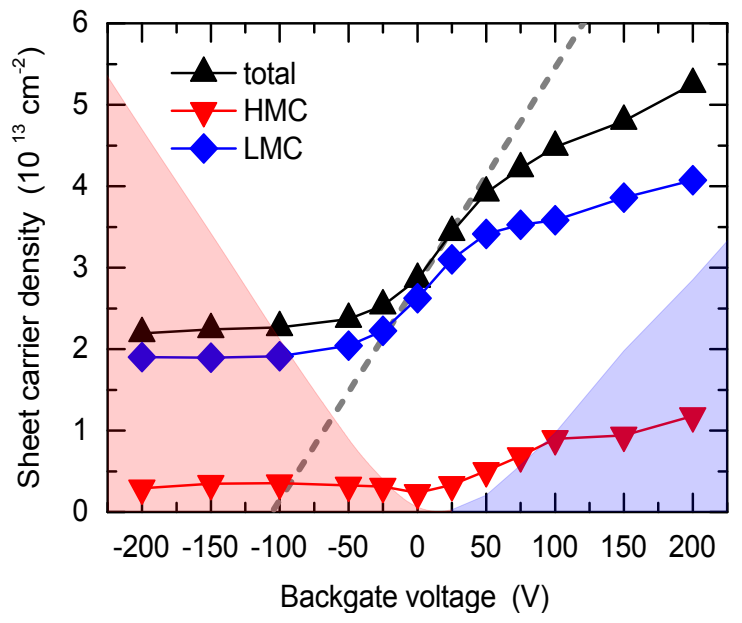

Figure 6.4: Magnetotransport characterization of the $15 \mathrm{~nm}$ thick film for different backgate voltages: (a) The magnitude of the WAL correction and the shape of $\Delta G$ change with the applied backgate bias. (b) the (antisymmetrized) Hall resistance as a function of backgate voltage: A small nonlinearity indicates multiband conduction. (c) The prefactor $\alpha$ and the dephasing length $l_{\phi}$ from a fit of the low-field magnetoconductance to the HLN equation. A clear change in the magnitude of the WAL correction appears in the transition region around $\sim 20 \mathrm{~V}$. The dephasing length also dips in this region. (d) The total sheet carrier density $n$ is estimated from a two band fit to the Hall resistance. In the transition region around $V_{m}$ athrmbg $\sim 20 \mathrm{~V}$, the change in total carriers $n_{t}$ follows the parallel plate capacitor relation [dashed grey line] with for a capacitor with the dielectric constant of STO at low temperatures $(\varepsilon \approx 24,000)$. The shaded areas on the bottom of the graph indicate deficit (blue) and excess carriers (red) compared to the parallel plate capacitor relation. (Inset) The background fit parameters, $c_{1}$ linear correction and $c_{2}$ the quadratic correction, used in the HLN fit: The quadratic contribution vanishes for $V_{\mathrm{bg}} \leq-100 \mathrm{~V}$. A small linear correction is needed only in the transition region. 
in the Josephson critical current previously. The dephasing length is found to be $\sim 0.8 \mu \mathrm{m}$ to either side of the transition but dips to $0.48 \mu \mathrm{m}$ in between. The linear $\left(c_{1}\right)$ and quadratic $\left(c_{2}\right)$ components of the background fit are plotted in the inset of Fig. 6.4(c). The linear correction is present only in the transition region. The quadratic component vanishes for $V_{\mathrm{bg}} \leq-100 \mathrm{~V}$.

We use a standard two-band model to fit the (antisymmetrized) Hall resistivity $\left(R_{x y}\right)$ [Fig. 6.4(b)]:

$$
R_{\mathrm{xy}}=-\left(\frac{B}{e}\right) \frac{n_{1} \mu_{1}^{2}+n_{2} \mu_{2}^{2}+B^{2} \mu_{1}^{2} \mu_{2}^{2}\left(n_{1}+n_{2}\right)}{\left(\left|n_{1}\right| \mu_{1}+\left|n_{2}\right| \mu_{2}\right)^{2}+B^{2} \mu_{1}^{2} \mu_{2}^{2}\left(n_{1}+n_{2}\right)^{2}},
$$

with the constraint $G^{-1}=e\left(n_{1} \mu_{1}+n_{2} \mu_{2}\right)$. The carrier densities $n_{1(2)}$, which correspond to carriers with mobilities $\mu_{1(2)}$, are shown in Fig. 6.4(d). We find carriers with higher mobility (HMC), $\mu_{1} \approx 1,900 \mathrm{~cm}^{2} \mathrm{~V}^{-1} \mathrm{~s}^{-1}$, and a lower mobility band (LMC) with $\mu_{2} \approx 720 \mathrm{~cm}^{2} \mathrm{~V}^{-1} \mathrm{~s}^{-1}$. The total carrier density, $n_{t}=n_{1}+n_{2}$, decreases gradually from $5.3 \times 10^{13} \mathrm{~cm}^{-2}$ (at $V_{\mathrm{bg}}=200 \mathrm{~V}$ ) to $2.2 \times 10^{13} \mathrm{~cm}^{-2}$ (at $-200 \mathrm{~V}$ ). In a narrow region around $V_{\mathrm{bg}}=20 \mathrm{~V}$, the charge carrier density follows the parallel plate capacitor relation for a capacitor with the (low temperature) dielectric constant of STO $(\varepsilon \approx 24,000)$. To either side of the transition region the density of mobile carriers deviates from this simple relationship. The colored regions in the bottom portion of Fig. 6.4(d) visualize this discrepancy.

\subsection{Discussion on unusual gate-dependence of Josephson supercurrent}

The central result of our study is the unusual gate bias dependence of the Josephson critical current in Fig. 6.3. A strong asymmetry between enhancement- and depletion-mode gating is observed in all our $\mathrm{Nb} / \mathrm{Bi}_{2} \mathrm{Te}_{3} / \mathrm{Nb}$ thin film Josephson devices. At small negative gate bias, the critical current flattens out at a large residual value whereas other transport quantities [e.g., the normal state resistance, $R_{N}$ ] change over the whole gate bias range. The gate voltage dependence of $I_{C}$ can be understood qualitatively by a comparison with the total carrier density $n_{t}$, extracted from the magnetotransport data of the 15 uc thick film [Fig. 6.4(d)]. Here, a large residual density of mobile, electronic carriers remains down to $-200 \mathrm{~V}$. The strong enhancement of $n_{t}$ around $V_{\mathrm{bg}} \approx 20 \mathrm{~V}$ and the somewhat slower, but linear, increase in carrier density at higher gate voltages is also reflected in the $I_{C}-V_{\mathrm{bg}}$ curve. Since the normal state resistance of the Josephson devices scales well with the (gate-dependent) sheet resistance of the material and the geometry of the weak links, the data suggests that the effect is driven by the (re)distribution of charge carriers in the TI material by the applied electric field. 
Recently we reported a carrier density modulation similar to Fig. 6.4(d) in capped $\mathrm{Bi}_{2} \mathrm{Te}_{3}$ thin films with low intrinsic carrier densities [46]. The additional carriers in the present samples are likely added by surface doping [i.e., vacancy formation and contaminations on the unprotected surface] during device fabrication. Therefore, we expect the dopants to be distributed nonuniformly in the material. Band bending occurs to ensure an equilibrium charge distribution with uniform electrochemical potential throughout the material. In case downward band bending [due to the accumulation of excess negative charge carriers] becomes strong enough, a quantum well forms, and one (or several) quantum well state(s) are populated in addition to the TSS. The intermediate carrier density $\left(\sim 10^{13} \mathrm{~cm}^{-2}\right)$ of our samples and the observed magnitude of the WAL correction support such a scenario [see below for a detailed discussion].

In this context, it is tempting to attribute the two carrier types of the two band model fit to TSS and quantum well bands. However, caution is warranted. Although such an approach can be instructive, e.g., in double-gating experiments on samples of quaternery compounds with very low intrinsic doping [64] when the TSS on the top and on the bottom surface can be addressed independently, we expect a more complex effective band structure for thin TI films with strong band bending. Hence our constrained fit to $R_{x y}$ yields only approximate values for the carrier densities and mobilities. To assign properties to the individual bands accurately, band structurerelated corrections must be modeled and taken into account when fitting both components of the resistivity matrix. Efforts to model the magnetoresistance of TI materials accurately are in progress.

The contribution of quantum well states to electric transport is supported qualitatively by the WAL data. Theory states that each (symplectic) conduction channel experiences a conductance correction $\sim 0.5 \frac{e^{2}}{h}$ from carrier localization [62]. Weak antilocalization is characterized by a conductance increase ( $\alpha=-0.5$ in the HLN formula). The effect is observed for the TSS where it arises from destructive wavefunction interference due to a Berry phase contribution of $\pi$ that is picked up by the interfering electrons. The additional phase is linked to the helical spin texture of the TSS. Quantum well bands with Rashba spin-splitting (from strong electric fields) have a similar texture and, thus, also exhibit the WAL effect. If the interchannel scattering time is longer than the characteristic time scale that leads to the carrier localization, the conductance corrections can add up to a larger weak (anti)localization signal. In the opposite case of strong scattering, we reduce the number of effective channels. Corrections due to electron-electron interactions or band structure topology also modify the localization effect [65]. At large positive gate voltages $\left(V_{\mathrm{bg}} \geq 75 \mathrm{~V}\right)$, the fit value $\alpha \approx-1.2$ and the long effective dephasing length $l_{\phi} \approx 0.8 \mu \mathrm{m}$ suggest the presence of two conduction channels in the sample. As the gate voltage is lowered, the magnitude of the (negative) WAL correction increases gradually to $\alpha \approx-1.7$. 
It flattens out at this value for $V_{\mathrm{bg}}<-100 \mathrm{~V}$. The dephasing length is significantly smaller in the transition region. The change in the WAL signal by $0.5 \frac{e^{2}}{h}$ can be interpreted either as the onset of interwell scattering between quantum well states associated with the top and the bottom surface, or strong band bending in the bottom region of the film shifts the bulk CBM below the Fermi level. It has been suggested that bulk bands exhibit weak localization with $\alpha \approx 0.5$ close to the bulk conduction band minimum [65] which would reduce the WAL signal in the present case. Both scenarios imply that (spatially) separated conduction channels exist, and that the scattering of charge carries between the different conducting regions changes significantly as we move through the gate bias range.

Despite the small sample thickness (both samples are thinner than the typical electrostatic screening length), a simple parallel plate capacitor relation (PPCR) holds only in a small region of the gate bias range [dashed line in Fig. 6.4(d)]. Here, most likely, carriers are added predominantly to the bottom surface $\left(\Delta n_{t} \approx 10^{13} \mathrm{~cm}^{-2}\right)$. A similar behavior has been observed in our previous work on capped $\mathrm{Bi}_{2} \mathrm{Te}_{3}$ thin films [46]. The observation that a strong deviation from the PPCR sets in when plateaux in the magnitude of the WAL signal [prefactor $\alpha$ in Fig. 6.4(c)] are reached hints at a band structure effect. We expect the electric field from the back gate to populate the TSS and the quantum well states of top and bottom surfaces at different rates that depend on the shape of the potential landscape in the bulk of the material. To understand this effect better, the charge distribution in the material must be obtained self-consistently. However, past a certain negative gate voltage the Josephson critical current becomes independent of the applied electric field. The scenario of several phase-coherent channels in the sample offers a plausible explanation for this effect. Andreev bound states (ABS) can only form in sample regions where phase-coherent transport is possible. As charge carriers are depleted from the bottom part of the film, the scattering probability between the superconducting electrodes on the top surface and the transport channels lower in the bulk diminishes. This effectively decouples the bottom part of the $\mathrm{TI}$, and ABS cannot form there. The residual supercurrent flows in the top portion of the device which shows only a week dependence on the applied electric field. Thus, $I_{C}$ remains essentially constant for negative gate voltage.

\subsection{Conclusion and outlook}

A comparison of the magnetotransport data of $\mathrm{TI}$ thin films and the gate-voltage dependence of the critical current in $\mathrm{Nb} / \mathrm{Bi}_{2} \mathrm{Te}_{3} / \mathrm{Nb}$ Josephson junctions reveals a strong link between supercurrent transport and the charge distribution in the sample. An analysis of the weak antilocalization 
Page 112 Chapter 6. Combined gate-tunable Josephson supercurrent and normal state ... effect provides evidence for the presence of several phase-coherent conduction channels. An externally applied electric field does not modulate the carrier density uniformly. Similarly, a finite critical current remains down to the lowest gate voltages. Besides the unusual backgate bias dependence of the Josephson supercurrent in $\mathrm{Nb}_{-}-\mathrm{Bi}_{2} \mathrm{Te}_{3}-\mathrm{Nb}$ junctions reported here, we also observed unconventional behaviours both in the response of the critical current to a perpendicularto-plane magnetic field and in the response of the Shapiro steps to the gate voltage. In chapter 7 , we will discuss both of these anomalous features. We will also suggest promising future directions towards the detection of Majorana zero energy bound states in Bi-based TI materials. 


\section{Bibliography}

[1] L. Fu and C. L. Kane, Phys. Rev. Lett. 100096407 (2008).

[2] Y. Tanaka, T. Yokoyama, and N. Nagaosa, Phys. Rev. Lett. 103, 107002 (2009).

[3] A. C. Potter and P. A. Lee Phys. Rev. B 83184520 ( 2011).

[4] C. Nayak, S. H. Simon, A. Stern, M. Freedman, and S. Das Sarma, Rev. Mod. Phys. 80, 1083 (2008).

[5] A. Stern, Nature 464, 187 (2010).

[6] V. Mourik, K. Zuo, S. M. Frolov, S. R. Plissard, E. P. A. M. Bakkers, and L. P. Kouwenhoven, Science 336, 1003 (2012).

[7] S. M. Albrecht, A. P. Higginbotham, M. Madsen, F. Kuemmeth, T. S. Jespersen1, J. Nygård, P. Krogstrup and C. M. Marcus, Nature 531, 206 (2016).

[8] A. D. K. Finck, D. J. Van Harlingen, P. K. Mohseni, K. Jung, and X. Li, Phys. Rev. Lett. 110, 126406 (2013).

[9] A. Das, Y. Ronen, Y. Most, Y. Oreg, M. Heiblum, and H. Shtrikman, Nat. Phys. 8, 887 (2012).

[10] H. O. H. Churchill, V. Fatemi, K. Grove-Rasmussen, M. T. Deng, P. Caroff, H. Q. Xu, and C. M. Marcus, Phys. Rev. B 87, 241401(R) (2013).

[11] M. T. Deng, C. L. Yu, G. Y. Huang, M. Larsson, P. Caroff, and H. Q. Xu, Nano Lett. 12, 6414 (2012).

[12] J. Wiedenmann, E. Bocquillon, R. S. Deacon, S. Hartinger, O. Herrmann, T. M. Klapwijk, L. Maier, C. Ames, C. Brüne, C. Gould, A. Oiwa, K. Ishibashi, S. Tarucha, H. Buhmann and L. W. Molenkamp, Nat. Commun. 7, 10303 (2016). 
[13] E. Bocquillon, R. S. Deacon, J. Wiedenmann, P. Leubner, T. M. Klapwijk, C. Brüne, K. Ishibashi, H. Buhmann, and L. W. Molenkamp, http://arxiv.org/abs/1601.08055 (2016).

[14] S. Nadj-Perge, I. K. Drozdov, J. Li, H. Chen, S. Jeon, J. Seo, A. H. MacDonald, B. A. Bernevig, and A. Yazdani, Science 346, 602 (2014).

[15] S. Nadj-Perge, I. K. Drozdov, B. A. Bernevig, and A. Yazdani, Phys. Rev. B 88, 020407 (2013).

[16] G. Tkachov and E. M. Hankiewicz, Phys. Rev. B 88, 075401 (2013).

[17] C. -K. Chiu, W. S. Cole, and S. Das Sarma, arXiv:1604.06105 (2016).

[18] M. Brahlek, N. Koirala, N. Bansal, S. Oh, Solid State Communications 215-216, 54 (2015).

[19] P. Ngabonziza, R. Heimbuch, N. de Jong, R. A. Klaassen, M. P. Stehno, M. Snelder, A. Solmaz, S. V. Ramankutty, E. Frantzeskakis, E. van Heumen, G. Koster, M. S. Golden, H. J. W. Zandvliet, and A. Brinkman, Phys. Rev. B 92, 035405 (2015).

[20] K. Hoefer, C. Becker, S. Wirth, and L. H. Tjeng, AIP Adv. 5, 097139 (2015).

[21] M.S. Bahramy, P.D.C King, A. de la Torre, J. Chang, M. Shi, L. Patthey, G. Balakrishnan, Ph. Hofmann, R. Arita, N. Nagaosa, and F. Baumberger, Nat. Commun. 3, 1159 (2012).

[22] D. Galanakis and Tudor D. Stanescu, Phys. Rev. B 86, 195311 (2012).

[23] Y. Maeno, H. Hashimoto, K. Yoshida, S. Nishizaki, T. Fujita, J. G. Bednorz and F. Lichtenberg, Nature 372, 532 (1994).

[24] A. P. Mackenzie and Y. Maeno, Rev. Mod. Phys. 75, 657 (2003).

[25] R. Joynt and L. Taillefer, Rev. Mod. Phys. 74, 235 (2002).

[26] Y. S. Hor, A. J. Williams, J. G. Checkelsky, P. Roushan, J. Seo, Q. Xu, H. W. Zandbergen, A. Yazdani, N. P. Ong, and R. J. Cava, Phys. Rev. Lett. 104, 057001 (2010).

[27] L. Fu and E. Berg, Phys. Rev. Lett. 105, 097001 (2010).

[28] B. Sacépé,, J. B. Oostinga, J. Li, A. Ubaldini, N. J. G. Couto, E. Giannini, and A. F. Morpurgo, Nat. Commun. 2, 575 (2011).

[29] J. R. Williams, A. J. Bestwick, P. Gallagher, S. S. Hong, Y. Cui, A. S. Bleich, J. G. Analytis, I. R. Fisher, and D. Goldhaber- Gordon, Phys.Rev.Lett. 109, 056803 (2012). 
[30] M. Veldhorst, M. Snelder, M. Hoek, T. Gang, V. K. Guduru, X. L. Wang, U. Zeitler, W. G. van der Wiel, A. A. Golubov, H. Hilgenkamp, and A. Brinkman, Nat. Mat. 11, 417 (2012).

[31] M. Veldhorst, C. G. Molenaar, X. L. Wang, H. Hilgenkamp, and A. Brinkman, Appl. Phys. Lett. 100, 072602 (2012).

[32] J. B. Oostinga, L. Maier, P. Schüffelgen, D. Knott, C. Ames, C. Brüne, G. Tkachov, H. Buhmann and L. W. Molenkamp, Phys. Rev. X 3021007 (2013).

[33] I. Sochnikov,A. J. Bestwick,J. R. Williams, . M. Lippman, I. R. Fisher,D. GoldhaberGordon,J. R. Kirtley and K. A. Moler, Nano Lett. 13, 3086 (2013).

[34] L. Galletti, S. Charpentier, M. lavarone, P. Lucignano, D. Massarotti, R. Arpaia, Y. Suzuki, K. Kadowaki, T. Bauch, A. Tagliacozzo, F. Tafuri, and F. Lombardi, Phys. Rev. B 89, $134512(2014)$.

[35] C. Kurter, A. D. K. Finck ,P. Ghaemi, Y. S. Hor and D. J. Van Harlingen, Phys. Rev. B 90, 014501 (2014).

[36] C. Kurter, A. D. K. Finck ,P. Ghaemi, Y. S. Hor and D. J. Van Harlingen, Nat. Commun. 6, 7130 (2015).

[37] F. Yang, F. Qu, J. Shen, Y. Ding, J. Chen, Z. Ji, G. Liu, J. Fan, C. Yang, L. Fu, and L. Lu, Phys.Rev.B 86, 134504 (2012).

[38] P. Zareapour, A. Hayat, S. Y. F. Zhao, M. Kreshchuk, A. Jain, D. C. Kwok, N. Lee, S. -W. Cheong, Z. Xu, A. Yang, G. D. Gu, S. Jia, R. J. Cava, and K. S. Burch, Nat. Commun. 3, 1056 (2012).

[39] C. G. Molenaar, D. P. Leusink, X. L. Wang and A. Brinkman, Supercond. Sci. Technol. 27, 104003 (2014).

[40] M. Snelder, C. G. Molenaar, Y. Pan,D. Wu, Y. K. Huang, A. de Visser, A. A. Golubov, W. G. van der Wiel, H. Hilgenkamp, M. S. Golden and A. Brinkman, Supercond. Sci. Technol. 27, 104001 (2014).

[41] L. Galletti, S. Charpentier, P. Lucignano, D. Massarotti, R. Arpaia, F. Tafuri, T. Bauchc, Y. Suzuki, A. Tagliacozzo, K. Kadowaki, and F. Lombardi, Physica C 503, 162 (2014).

[42] M. P. Stehno, V. Orlyanchik, C. D. Nugroho, P. Ghaemi, M. Brahlek, N. Koirala, S. Oh, and D. J. Van Harlingen, Phys. Rev. B 93, 035307 (2016). 
[43] I. Sochnikov, L. Maier, C. A. Watson, J. R. Kirtley, C. Gould, G. Tkachov, E. M. Hankiewicz, C. Brüne, H. Buhmann, L. W. Molenkamp and K. A. Moler, Phys. Rev. Lett. 114, 066801 (2015).

[44] C. T. Olund and E. Zhao, Phys. Rev. B 86, 214515 (2012).

[45] M. Snelder, A. A. Golubov, Y. Asano and A. Brinkman, J. Phys.: Condens. Matter 27, 315701 (2015).

[46] P. Ngabonziza, M. P. Stehno, H. Myoren, V. A. Neumann, G. Koster, and A. Brinkman, Adv. Electron. Mater. 2, 1600157 (2016).

[47] T. Chen, Q. Chen, K. Schouteden, W. Huang, X. Wang, Z. Li, F. Miao, X. Wang, Z. Li, B. Zhao, S. Li, F. Song, J. Wang, B. Wang, C. Van Haesendonck, and G. Wang, Nat. Commun. 75, 5022 (2014).

[48] M. Brahlek, N. Koirala, M. Salehi, N. Bansal, and S. Oh, Phys. Rev. Lett. 113, 026801 (2014).

[49] M. Lanius, J. Kampmeier, C. Weyrich, S. Koölling, M. Schall, P. Schüffelgen, E. Neumann, M. Luysberg, G. Mussler, P. M. Koenraad, T. Schäpers, and D. Grützmacher, Cryst. Growth Des. 16, 2057 (2016).

[50] M. Eschbach, E. Młyńczak, J. Kellner, J. Kampmeier, M. Lanius, E. Neumann, C. Weyrich, M. Gehlmann, P. Gospodaric, S. Döring, G. Mussler, N. Demarina, M. Luysberg, G. Bihlmayer, T. Schäpers, L. Plucinski, S. Blügel, M. Morgenstern, C. M. Schneider, and D. Grützmacher, Nat. Comm. 6 (2016).

[51] J. Wang, X. Chen, B. -F. Zhu, and S. -C. Zhang, Phys. Rev. B 85, 235131 (2012).

[52] J. Zhang, C. Z. Chang, Z. Zhang, J. Wen, X. Feng, K. Li, M. Liu, K. He, L. Wang, X. Chen, Q. K. Xue, X. Ma, Y. Wang, Nat. Commun. 2, 574 (2011).

[53] Y. Xu, I. Miotkowski, C. Liu, J. Tian, H. Nam, N. Alidoust, J. Hu, C. -K. Shih, M. Z. Hasan, and Y. P. Chen, Nat. Phys. 10, 956 (2014).

[54] T. Arakane, T. Sato, S. Souma, K. Kosaka, K. Nakayama, M. Komatsu, T. Takahashi, Z. Ren,K. Segawa, and Y. Ando, Nat. Commun. 3, 636 (2012).

[55] N. Bansal, Y. S. Kim, M. Brahlek, E. Edrey, and S. Oh, Phys. Rev. Lett. 109, 116804 (2012). 
[56] P. D. C. King, R. C. Hatch, M. Bianchi, R. Ovsyannikov, C. Lupulescu, G. Landolt, B. Slomski, J. H. Dil, D. Guan, J. L. Mi, E. D. L. Rienks, J. Fink, A. Lindblad, S. Svensson, S. Bao, G. Balakrishnan, B. B. Iversen, J. Osterwalder, W. Eberhardt, F. Baumberger, and Ph. Hofmann, Phys. Rev. Lett. 107, 096802 (2014).

[57] M. Bianchi, D. Guan, S. Bao, J. Mi, B. B. Iversen, P. D.C. King, and P. Hofmann, Nat. Comm. 1, 128 (2010).

[58] Y. Zhang, K. He, C. Z. Chang, C. L. Song, L. L. Wang, X. Chen, J. F. Jia, Z. Fan, X. Dai, W. Y. Shan, S. Q. Shen, Q. Niu, X. L. Qi, S. C. Zhang, X. C. Ma and Q. K. Xue, Nat. Phys. 6, 584 (2010).

[59] C. -X. Liu, H. Zhang, B. Yan, X. -L. Qi, T. Frauenheim, X. Dai, Z. Fang, and S. -C. Zhang, Phys. Rev. B 81, 041307(R) (2010).

[60] G. S. Jenkins and D. C. Schmadel, Phys. Rev. B 87, 155126 (2013).

[61] S. Shapiro, Phys. Rev. Lett. 11, 80 (1963).

[62] S. Hikami, A. I. Larkin and Y. Nagaoka, Prog. Theor. Phys. 63, 707 (1980).

[63] S. Maekawa, and H. Fukuyama, J. of the Phys. Soc. of Japan 50, 2516 (1981).

[64] J. Lee, J. Park, J. -H. Lee, J. S. Kim, and H. -J. Lee, Phys. Rev. B 86, 245321 (2012).

[65] H. -Z. Lu and S. -Q. Shen, Phys. Rev. Lett. 112, 146601 (2014). 



\section{7}

\section{Future directions}

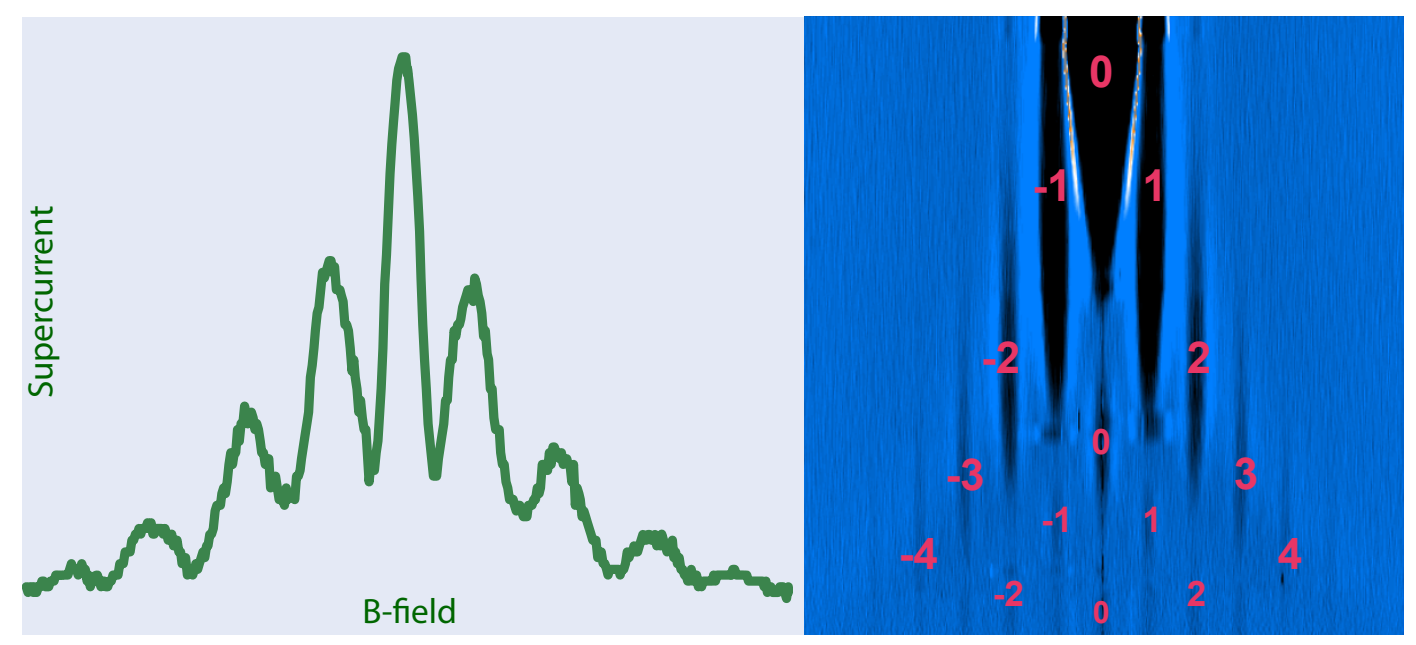

In chapter 6, we discussed the critical current and normal state transport behaviour in bottom-gated $\mathrm{Nb} / \mathrm{Bi}_{2} \mathrm{Te}_{3} / \mathrm{Nb}$ topological insulator Josephson devices (TIJDs) and Hall bar structures fabricated sideby-side on $\mathrm{Bi}_{2} \mathrm{Te}_{3}$ thin films. Here, we provide an epilogue of our work on $\mathrm{Nb} / \mathrm{Bi}_{2} \mathrm{Te}_{3} / \mathrm{Nb}$ TIJD and future directions are suggested. First, we show preliminary experimental indications of supercurrent originating from states probably located on the edges of a junction fabricated on a $6 \mathrm{~nm} \mathrm{Bi}_{2} \mathrm{Te}_{3}$ thin film. To unveil the periodicity of the Josephson supercurrent, Shapiro steps were measured at different back-gate voltages. We observe anomalous behaviour in Shapiro steps appearing at certain back-gate voltages. This chapter ends with an outlook on future experiments that should focus on first identifying under which thickness range, the $\mathrm{Bi}_{2} \mathrm{Te}_{3}$ thin films are in the $\mathrm{QSH}$ regime; and then fabricate on them highly tunable dual-gated TIJDs based on in-situ capped samples. 


\subsection{Preliminary sign of edge supercurrents in $\mathrm{Nb}-\mathrm{Bi}_{2} \mathrm{Te}_{3}-\mathrm{Nb}$ topological Josephson devices}

A crossover regime from $3 \mathrm{D}$ to $2 \mathrm{D} \mathrm{TI}$ phase is anticipated in the band structure of $\mathrm{Bi}_{2} \mathrm{Se}_{3}$ and $\mathrm{Bi}_{2} \mathrm{Te}_{3} \mathrm{TI}$ materials, due to hybridization effects, when the sample thickness is reduced $[1,2]$. In such $2 \mathrm{D} \mathrm{TI}$ phase, the sample would be in the 2D quantum spin Hall (QSH) regime, with the current carried solely by the helical edge states. A promising route towards the realization of zero-energy Majorana modes in 3D Tls, particularly in Bi-based Tls, is to use the 1D edge states originating from thin films which are in quantum spin Hall (QSH) regime. For such systems in 2D TI regime, electric current flows near the edges of the sample and is protected against elastic backscattering by time-reversal symmetry $[3,4]$. As discussed in recent theoretical proposals $[5,6]$, TIJDs fabricated on such 2D TI samples act as nanoscale SQUIDs (superconducting quantum interference devices) in which a magnetic flux enclosed in the interior of the $2 \mathrm{D} \mathrm{TI}$ controls the interference of the Josephson currents flowing at the opposite edges of the sample. To date, proximity-induced superconductivity in the QSH edge has only been reported in $\mathrm{HgTe} / \mathrm{CdTe}$ and InAs/GaSb quantum wells [7, 8]. It would be good, on the other hand, to look for such features in TIJDs fabricated on bulk insulating thinner Bi-base TI samples in QSH regime. In this regime, trivial non-perpendicular channels in the junctions, contributing gapped $2 \pi$-periodic modes Andreev bound states, would be significantly reduced and the Majorana fermions fingerprints would then appear strongly [9].

To probe quantum interference of edge supercurrents in Bi-based 2D TI, we fabricated TIJDs on $6 \mathrm{~nm} \mathrm{Bi}_{2} \mathrm{Te}_{3}$ thin film grown by molecular beam epitaxy (MBE) on $\mathrm{SrTiO}_{3}$ [111] substrate. We use the $6 \mathrm{~nm}$ thin film because around this thickness regime, the sample is close to a crossover from 3D to 2D TI phase in the band structure $[1,2]$. The thin film quality, Josephson junction characteristics of devices prepared on top of this $6 \mathrm{~nm} \mathrm{Bi} \mathrm{Te}_{3}$ sample, and details on fabrications steps are described in the previous chapter 6 . Using the $\mathrm{SrTiO}_{3}$ [111] substrate as a gate dielectric, we tune the carrier density electrostatically and study the evolution of the diffraction pattern, i.e dependence of the critical current on magnetic field.

Applying a perpendicular magnetic field to Josephson junctions results in a "Fraunhofer-like" magnetic diffraction pattern due to the d.c. Josephson effect. For the simplest case, in limit of infinite width and a homogeneous current density distribution in the Josephson junction, the magnetic field dependence of the critical current follows a regular Fraunhofer sinc function $[10,11]$

$$
I_{c}\left(\Phi_{0}\right)=I_{c}(0) \frac{\sin \left(\pi \Phi / \Phi_{0}\right)}{\pi \Phi / \Phi_{0}}
$$




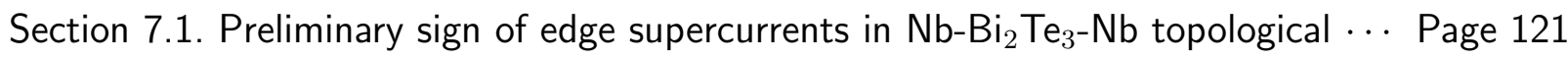

(a)

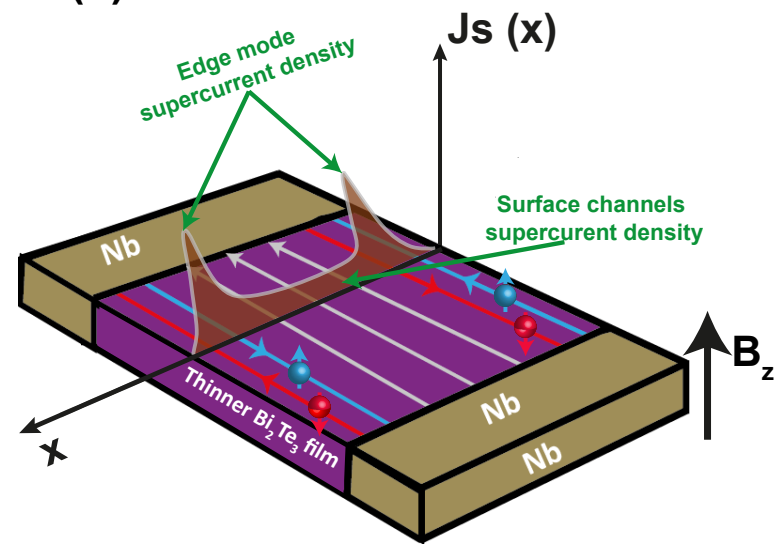

(b)

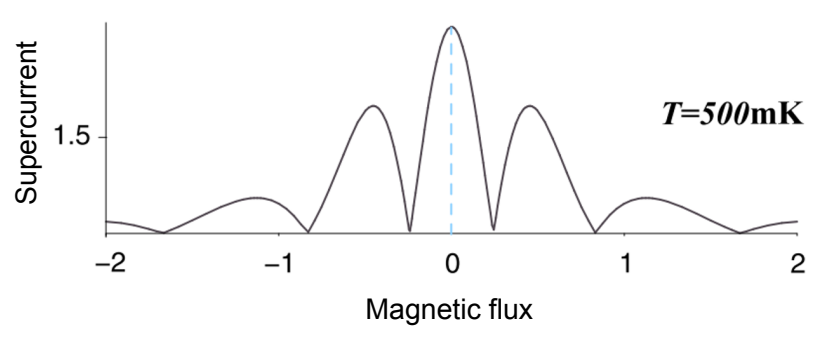

(c)

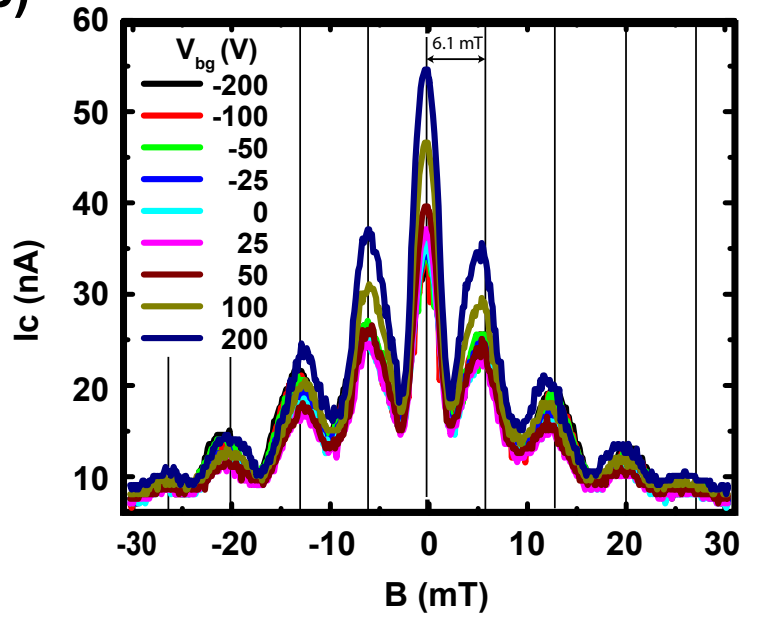

Figure 7.1: Signature of quantum interference of edge supercurrents in thin $\mathrm{Bi}_{2} \mathrm{Te}_{3} \mathrm{TI}$ thin film. (a) Schematic illustration of a TIJD with reduced bulk carriers. The supercurrent density start to develop peaks owing to the presence of the helical edges, signalling an evolution towards edge-dominated transport. In this regime, a moderate current still flows "uniformly" in the 2D plane of the TIJD. (b) Theoretical predicted behaviour of supercurrent versus magnetic flux in an edge-dominated supercurrent regime (Fig. from Ref. [5]). (c) Critical current dependence on the magnetic field, applied perpendicularly to the junction plane, measured at different back-gate voltage.

where $I c(0)$ is the critical current of the junction at zero magnetic field, and $\Phi$ the flux through the junction area. Studying the critical current, modulated by the magnetic flux with a periodicity given by the superconducting flux quantum $\left(\Phi_{0}=h / 2 e\right)$, will then reveal the properties of the spatial distribution of the supercurrent. For topologically non-trivial Josephson junctions, the periodicity is expected to become $h / e$. This is because the associated current-phase relationship is changed from $\sin (\varphi)$ to $\sin (\varphi / 2)$ due to the presence of topologically protected zero-energy 
bound states, carrying a $4 \pi$-periodic supercurrent $\left(I_{4 \pi} \sin \varphi / 2\right)[12]$.

Figure 7.1(a) depicts a schematic illustration of a two-terminal Josephson junction, with a rect-

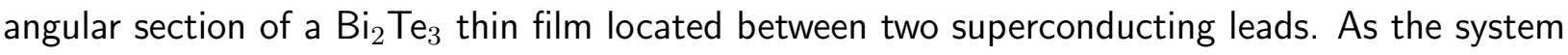
approaches the crossover regime (from $3 \mathrm{D} \mathrm{TI}$ to the $2 \mathrm{D}$ limit), depending on the surface channels density, the appearance or absence of helical edge channels in this device will influence the supercurrent density profile across the junction width. By decreasing the density of the surface channels, the TIJD will be brought closer to the QSH insulator regime. In the 2D limit, the helical edge states contribution emerges as peaks in the supercurrent density at each edge of the TIJD. Such a evolution towards an edge- dominated supercurrent appears in the interference pattern as a narrowing central lobe width and more pronounced side-lobe amplitudes [5, 6] (see Fig.7.1(b)). When the $2 \mathrm{D}$ channels are significantly reduced, the edge state peaks become pronounced since the supercurrent will be carried solely along the helical edges. In this regime, the TIJD becomes a quantum spin Hall superconductor, and the interference pattern develop sinusoidal double-slit behaviour, like oscillations reminiscent of the SQUID pattern rather than the Fraunhofer pattern expected in non-TIJDs $[5,6]$.

In Fig. 7.1(c), we present the measured response of the supercurrent to a perpendicular magnetic field, for different back-gate voltages $\left(V_{b g}\right)$, in the $6 \mathrm{~nm} \mathrm{Bi}_{2} \mathrm{Te}_{3}$ TIJD. The data were acquired when sweeping $V_{b g}$ from positive to negative values. For all $V_{b g}$ values, our TIJD exhibits unconventional Fraunhofer pattern behaviour. As $V_{b g}$ is decreased, the critical current decreases without changing the overall behaviour. We extract a period of the interference maxima of $6.1 \mathrm{mT}$. Given the dimensions of the device $(L=250 \mathrm{~nm}, W=860 \mathrm{~nm})$, the area of the junction is given by [13]: $W(L+2 \lambda)$, with $\lambda$ being the penetration length. We extract a penetration length ${ }^{1}$ of $\lambda \simeq 70$ $\mathrm{nm}$, which is in the range of previously reported values $[14,15]$. Thus, the maxima are attributed to the points where the magnetic flux $\Phi$ through the junction is a multiple of the flux quantum $\Phi_{0}$. For a system in the QSH regime with edge supercurrents, the magnetic flux maxima would be integer multiples of the flux quantum: $\Phi=n \Phi_{0}, n \in \mathbb{Z}$, and exhibiting the (dc) SQUID-like diffraction pattern behaviour $[5,6,7]$. The measured shift towards a SQUID-like interference pattern in the $6 \mathrm{~nm}$ TIJD suggests a development of peaks in supercurrent density at both edges, coexisting with residual two-dimensional current flow in the junction. The observed non-zero $I_{c}(B)$ are most likely related to an asymmetry in the supercurrent distribution along the edges of the junction [7, 17], although other effects might be the origin of non-zero minima in diffraction patterns $[18,19,22]$.

\footnotetext{
${ }^{1}$ Here, we used the superconducting magnetic flux quantum with periodicity $\Phi_{0}=h / 2 e$. Assuming a $\sin (\varphi / 2)$ current-phase relationship, the periodicity would be $[12,20,21] h / e$, and we extract a penetration length of $\lambda \simeq 270 \mathrm{~nm}$, which would also be realistic given previously reported values $[13,16,17,23,24,25]$.
} 


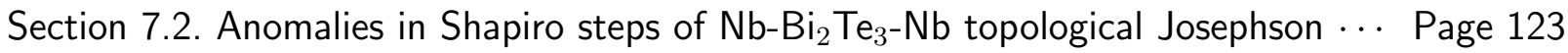

The measured peculiarities in the $I_{c}(B)$ at different back-gate voltage provide a promising sign towards the formation edge-mode proximity-induced superconductivity in thin $\mathrm{Bi}_{2} \mathrm{Te}_{3}$ TIJDs with QSH behaviour. Such regime is proposed to be the ideal experimental configuration to detect Majorana bound states $[5,6,9]$. This work is in progress, and it is based on first identifying under which thickness range the $\mathrm{Bi}_{2} \mathrm{Te}_{3}$ thin films are in the QSH regime. In the literature, it is predicted that an oscillatory crossover from 3D TI to $2 \mathrm{D}$ TI regime will happen in $\mathrm{Bi}_{2} \mathrm{Te}_{3}$ thin films with quantum spin Hall effect, when the sample thickness is decreased [1]. The homogeneity of the desired MBE grown ultra-thin films should be high so that all the regions of the sample will be in the QSH regime and to avoid an asymmetry in supercurrent distribution of the subsequently fabricated TIJDs.

\subsection{Anomalies in Shapiro steps of $\mathrm{Nb}-\mathrm{Bi}_{2} \mathrm{Te}_{3}-\mathrm{Nb}$ topological Josephson junctions}

In the presence of $\mathrm{rf}$ radiation, current plateaux appear in the I-V characteristics of the junction, with zero differential resistivity, for voltages $V_{n}=n h f / 2 e \simeq 12.4 \mu \mathrm{V}$. Here, $n, h, f$ and $e$ are the step index $n \in \mathbb{Z}$, the Planck's constant, rf frequency, and the elementary charge, respectively. These plateaux, called Shapiro steps [26], are due to the AC Josephson effect, and are one of the hallmarks of the Josephson coupling in superconductor-normal metal junctions. Unlike the Fraunhofer-like magnetic diffraction patterns, which depend on the geometry of the junction, Shapiro steps do not; they rather depend on the current-phase relation [27]. For a pure $\sin (\varphi / 2)$ current-phase relationship, contributing a $4 \pi$-periodic supercurrent $[12,28,29]$, an unconventional sequence of even steps, with missing odd steps, is expected.

To probe the current-phase characteristics and look for anomalous response to rf irradiation (with $f=6.02 \mathrm{GHz}$ ) in TIJD fabricated on $\mathrm{Bi}_{2} \mathrm{Te}_{3}$ thin films, we study the evolution of the Shapiro steps in the back-gate voltage range of $\pm 200 \mathrm{~V}$. We back-gated the devices using the $\mathrm{SrTiO}_{3}$ [111] substrate as a gate dielectric. Below, we detail our experimental observations.

We first present the measured anomalous features in the dependence of the Shapiro steps response on the back-gate voltage, for a weak link made of a $6 \mathrm{~nm} \mathrm{Bi} \mathrm{Bi}_{2} \mathrm{Te}_{3}$ structure. Figure 7.2 gives differential resistance maps after irradiation with microwaves of frequency $f=6.02 \mathrm{GHz}$, for different $V_{b g}$ values. The maps were acquired starting from $V_{b g}=+200 \mathrm{~V}$ going down to negative $V_{b g}$ values (up to $V_{b g}=-200 \mathrm{~V}$ ). Shapiro steps are observed for all $V_{b g}$ values (see the regions encoded by black in the color-scale map in Fig. 7.2) when the junction is irradiated 


\section{$6 \mathrm{~nm} \mathrm{Nb}-\mathrm{Bi}_{2} \mathrm{Te}_{3}-\mathrm{Nb}$ Josephson junction}
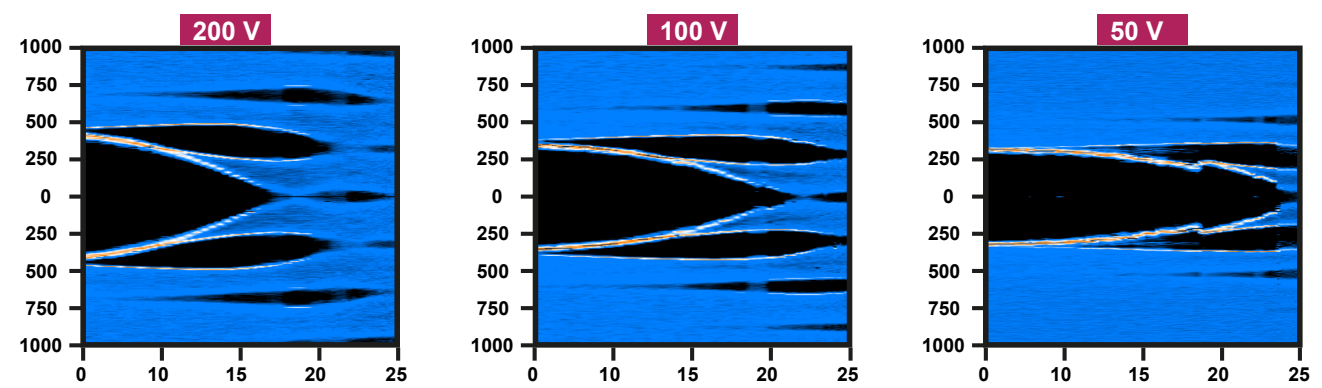

$\mathrm{dV} / \mathrm{dI}(\Omega)$
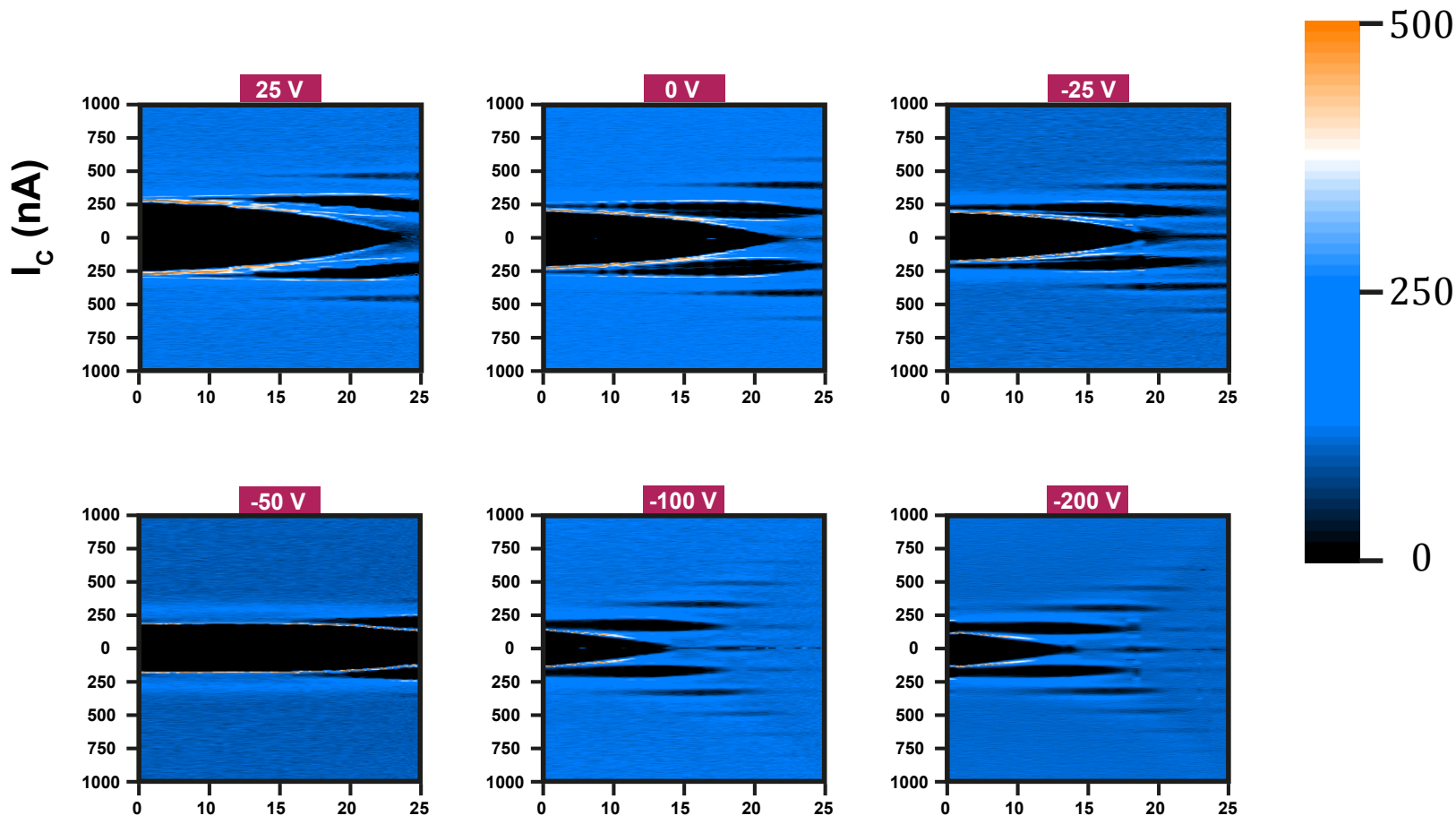

\section{RF power $(\mathrm{dBm})$}

Figure 7.2: Color-scale plots of the differential resistance $(d V / d I)$, as a function of the $\mathrm{DC}$ and $\mathrm{rf}$ current drives $I$ and $I_{r f}$, respectively, for different back-gate voltages (from $V_{b g}=+200 \mathrm{~V}$ down to $\left.V_{b g}=-200 \mathrm{~V}\right)$. A microwave frequency of $f=6.02 \mathrm{GHz}$ was used. Data are from a TIJD fabricated on a $6 \mathrm{~nm} \mathrm{Bi}_{2} \mathrm{Te}_{3}$ thin film. The black regions in the color-scale maps correspond to the voltage plateaux, in the $\mathrm{V}$-I curves under microwave irradiation (see Fig. 6.2(d) for corresponding $\mathrm{V}$-I curves at $\left.V_{b g}=0 \mathrm{~V}\right)$.

with microwaves. Analysing I-V curves extracted from the color-scale maps, the spacing between Shapiro steps show that both even and odd $n$ steps are present at the employed rf frequency. At large positive gate voltage $\left(V_{b g}=+200 \mathrm{~V}\right)$, the maximum critical current of the first Shapiro step is $I_{c}^{0}=380 \mathrm{nA}$ at the lowest drive amplitude. The measured Shapiro steps modulations, at this back-gate voltage, qualitatively agrees with the conventional $\sin \varphi$ current phase relation. 


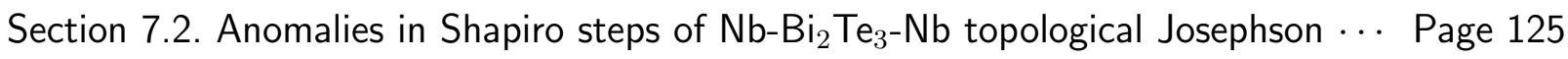

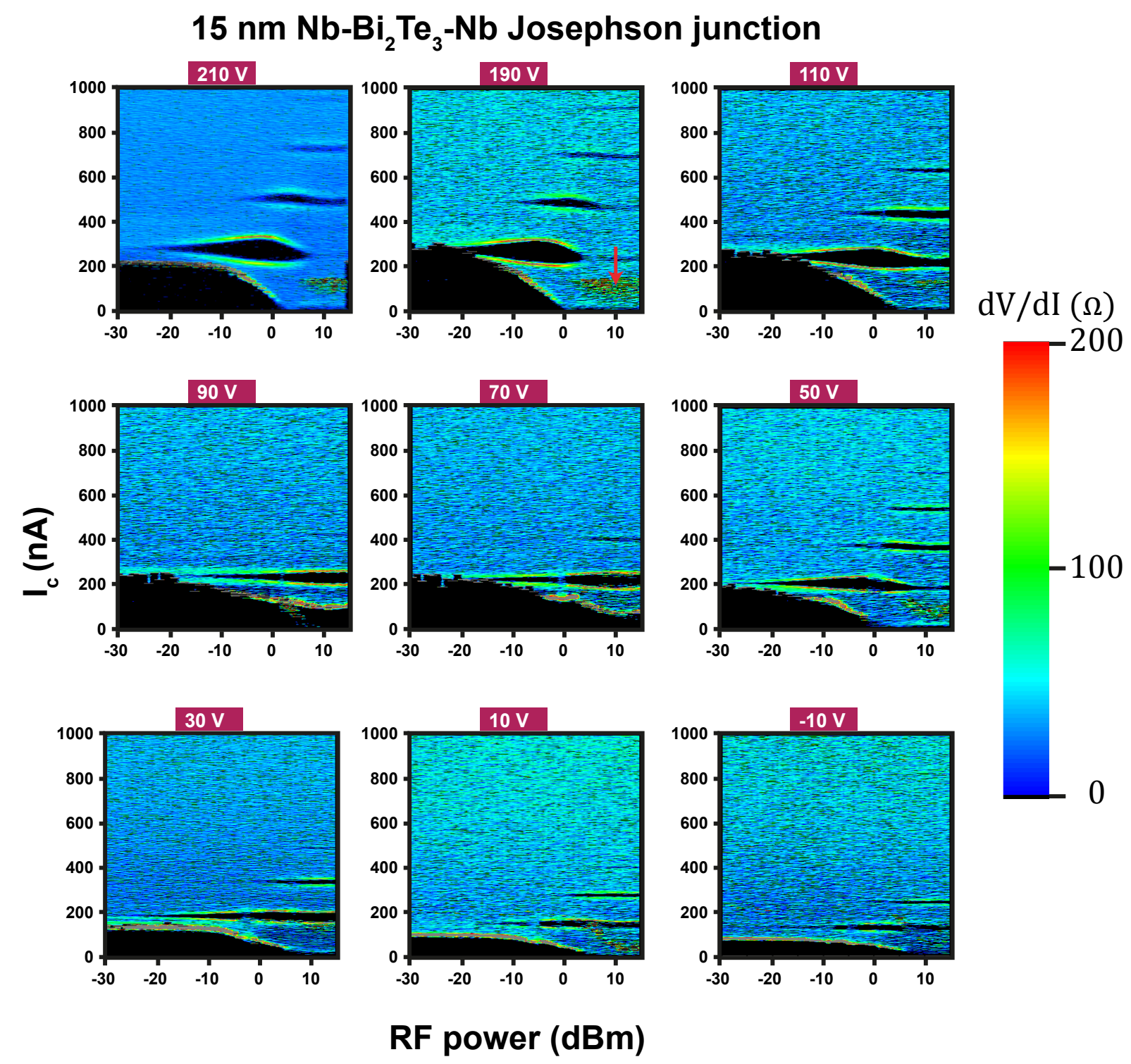

Figure 7.3: Color-scale maps of the differential resistance $(d V / d I)$, as a function of the $\mathrm{DC}$ and rf current drives $I$ and $I_{r f}$, respectively, for different back-gate voltages (from $V_{b g}=+210 \mathrm{~V}$ down to $V_{b g}=-10 \mathrm{~V}$ ). A microwave frequency of $f=6.02 \mathrm{GHz}$ was used. Data are from a TIJD fabricated on a $15 \mathrm{~nm} \mathrm{Bi} \mathrm{Te}_{3}$ thin film. The red arrow at $V_{b g}=190 \mathrm{~V}$ indicates the presence of subharmonic step in the Shapiro response.

This observation is also in agreement with the $V_{b g}$ dependence of the critical current, $I_{c}$, since at $V_{b g}=200 \mathrm{~V}$ we are in the bulk-dominated regime with a single conduction channel, where the coupling is caused by a conducting bulk. As $V_{b g}$ is lowered, we start to observe unusual response to $\mathrm{rf}$ irradiation in the Josephson junction. At $V_{b g}=+100 \mathrm{~V}$, the critical current drops down to $I_{c}^{0}=330 \mathrm{nA}$; but unexpectedly, the maximum rf power increases for the (first) Shapiro 
steps. Usually, one would anticipate, for a conventional $\sin \varphi$ current phase relation, that the rf drive current $I_{r f}$ would decrease as the $I_{c}$ becomes smaller. This unusual trend becomes even more pronounced in the intermediate back-gate voltage regime: $-50 \mathrm{~V} \leq V_{b g} \leq+50 \mathrm{~V}$. In this intermediate region, the $I_{c}-V_{b g}$ of this same device was observed to decrease rapidly, while the resistance increased strongly in the same $V_{b g}$ window of $\sim 100 \mathrm{~V}$ wide (see Fig. 6.3(a)). As discussed in chapter 6 , there are probably two or three conduction channels, which are distributed between the top and bottom surface. These channels are most likely decoupled by the depletion zone in the center region of the $\mathrm{Tl}$ material, and the supercurrent distribution follows a much more complicated current-phase relation ${ }^{2}$. At large negative gate voltages $\left(V_{b g} \leq-100\right) \mathrm{V}$, the Shapiro steps response on the $V_{b g}$ appear to exhibit again the conventional behaviour. At these large negative gate voltages, we are in the regime where most of the supercurrent is most likely carried by the top surface (see Fig. 6.3(a) in chapter 6 and related discussion); and it would be reasonable in that situation to observe conventional Shapiro step behaviour at this frequency ( $f=6.02 \mathrm{GHz}$ ), as observed in other $\mathrm{TI}$ samples $[15,25]$. A more detailed theoretical understanding and experimental investigation of the observed anomalous Shapiro steps response to the gate voltage is needed in order to understand better their associated current-phase relationship at different gate voltages.

Next, we present the dependence of the Shapiro steps response on the back-gate voltage, for a TIJD fabricated on a $15 \mathrm{~nm} \mathrm{Bi} \mathrm{Te}_{3}$ thin film ${ }^{3}$. In Fig. 7.3, we plot of color maps of $d V / d I$, at different gate voltages, after irradiation with a microwave frequency of $f=6.02 \mathrm{GHz}$. The bottom-gate was swept from $210 \mathrm{~V}$ down to $-10 \mathrm{~V}$. For this sample also, in the intermediate backvoltage region ( $90 \mathrm{~V} \leq V_{b g} \leq-10 \mathrm{~V}$ ) we observe an anomalous response (the critical current drops down, but the maximum rf power of the first Shapiro step increases) to rf irradiation in the Josephson junction. This anomalous behaviours, even though they are less pronounced when compared to the $6 \mathrm{~nm}$ sample, are also attributed to decoupled conducting channels, distributed between the top and bottom surface (a detailed discussion on possible transport regimes present in this sample is presented in section 6.4 of chapter 6 ). Another noticeable unusual feature, for different $V_{b g}$ regime, is the fact that there are additional subharmonic steps in the Shapiro response of the gate voltage (a typical example is indicated by a red arrow in Fig. 7.3 for $V_{b g}=190 \mathrm{~V}$ ). Subharmonic steps in Shapiro response indicate a nonsinusoidal current phase relationship in the Josephson Junction, and they have been observed previously in junctions fabricated on strained

\footnotetext{
${ }^{2}$ Very speculatively, it is also tempting to attribute this behaviour to the presence of decoupled 1D edge modes, living on both edges of the sample, a scenario that would be consistent with the measured magnetic diffraction pattern (see Fig. 7.1(c).)

${ }^{3}$ The same sample on which we discussed gate-dependence of the supercurrent and the normal state transport in the previous chapter 6 .
} 
(a)

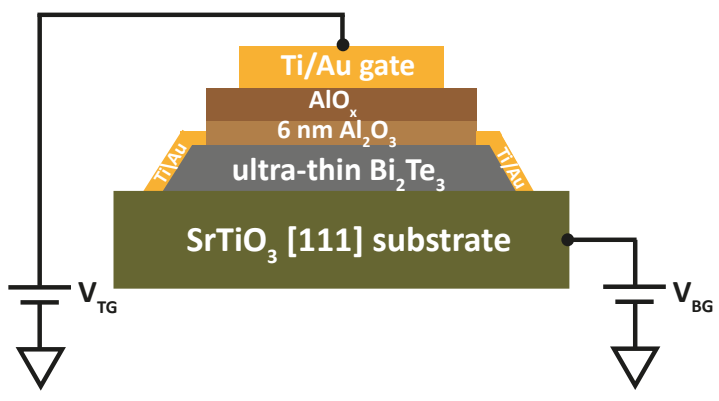

(b)

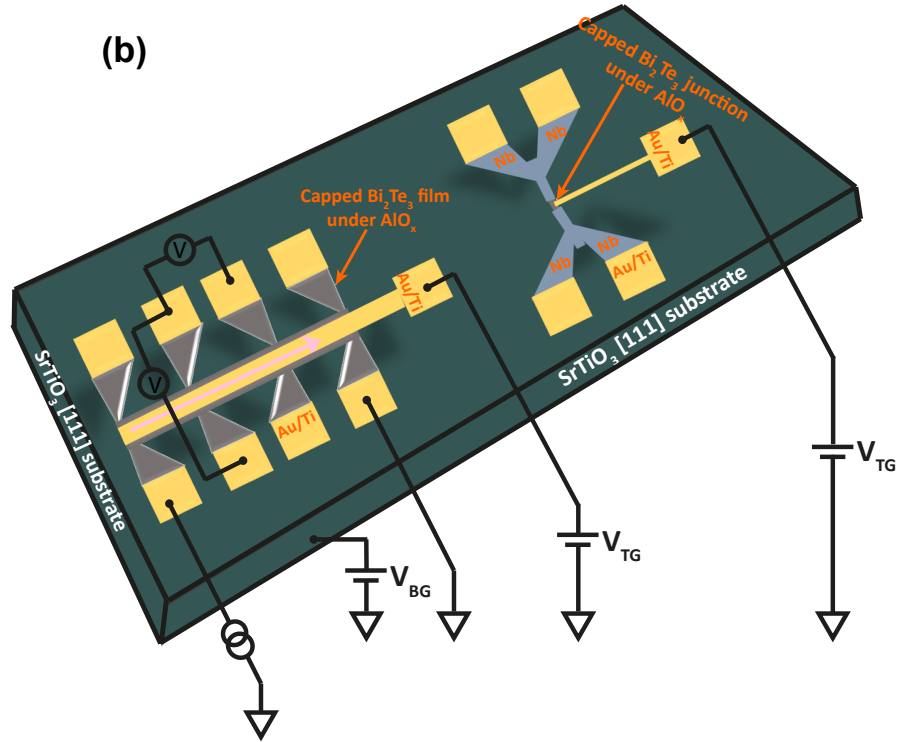

Figure 7.4: Dual-gating configuration in $\mathrm{Bi}_{2} \mathrm{Te}_{3}$ thin films. (a) Schematics of the envisaged thinner $\mathrm{Bi}_{2} \mathrm{Te}_{3}$ sample, which is capped in-situ. Using both the top and bottom-gates, the sample can be gated towards the quantum spin Hall regime. (b) Illustrative image of a topological insulator Josephson device and a Hall bar structure fabricated side-by-side on the same $\mathrm{Bi}_{2} \mathrm{Te}_{3}$ thin film. The measurement configuration is also illustrated.

$\mathrm{HgTe} \mathrm{TI}$ samples [16, 30] and other non-TI materials [31, 32]. Such subharmonic behaviours have been previously attributed to non-linearities, capacitance effects or higher harmonics in the current phase relationship [33,34]. These subharmonic Shapiro steps anomalies were observed in one sample, a more elaborate investigation of this feature is needed in order to understand their origin and contribution to the current phase relation.

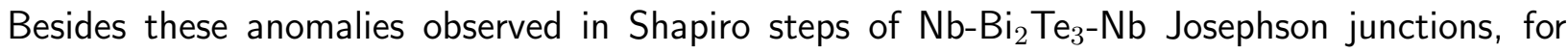
future experiments, it would be good to investigate the Shapiro step response to the gate voltage for TIJDs fabricated on thinner $\mathrm{Bi}_{2} \mathrm{Te}_{3}$ films in the quantum spin Hall effect regime. In such regime, the total number of transport modes should be reduced to a single channel regime, which would be rather the ideal experimental configuration to detect Majorana bound states signature in $\mathrm{Nb}_{-} \mathrm{Bi}_{2} \mathrm{Te}_{3}-\mathrm{Nb}$ Josephson junctions. It is suggested to grow such thin films on insulating substrates, with high relative dielectric constant, to fabricate an efficient top gate on them and to cap them in-situ before exposure to ambient conditions to avoid their degradation (see Fig. 7.4(a) and 7.4(b)). The dual-gate configuration of these devices will be useful in pushing the sample towards the quantum spin Hall regime and investigating both the Shapiro steps and magnetic diffraction pattern response to the gate voltage. 


\section{Bibliography}

[1] C. -X. Liu, H. Zhang, B. Yan, X. -L. Qi, T. Frauenheim, X. Dai, Z. Fang, and S. -C. Zhang, Phys. Rev. B 81, 041307(R) (2010).

[2] Y. Zhang, K. He, C. Z. Chang, C. L. Song, L. L. Wang, X. Chen, J. F. Jia, Z. Fan, X. Dai, W. Y. Shan, S. Q. Shen, Q. Niu, X. L. Qi, S. C. Zhang, X. C. Ma and Q. K. Xue, Nat. Phys. 6, 584 (2010).

[3] M. Z. Hasan and C. L. Kane, Rev. Mod. Phys. 82, 3045 (2010).

[4] X. -L. Qi and S. -C. Zhang, Rev. Mod. Phys. 83, 1057 (2011).

[5] G. Tkachov, P. Burset, B. Trauzettel, and E. M. Hankiewicz, Phys. Rev. B 92, 045408 (2015).

[6] J. Song, H. Liu, J. Liu, Y. Li, R. Joynt, Q. -F. Sun, and X. C. Xie, Phys. Rev. B 93, 195302 (2016).

[7] S. Hart, H. Ren, T. Wagner, P. Leubner, M. Mühlbauer, C. Brüne, H. Buhmann, L. W. Molenkamp and A. Yacoby, Nat. Phys. 10, 638 (2014).

[8] V. S. Pribiag, A. J. A. Beukman, F. Qu, M. C. Cassidy, C. Charpentier, W. Wegscheider, and L. P. Kouwenhoven, Nat. Nanotechnol. 10, 593 (2015).

[9] M. Snelder, M. Veldhorst, A. A. Golubov, and A. Brinkman, Phys. Rev. B 87, 104507 (2013).

[10] A. Barone and G. Paterno, Physics and applications of the Josephson effect, John Wiley \& Sons, New York, USA (1982).

[11] J. Clarke and A. I. Braginski (Eds.), The SQUID Handbook: Fundamentals and Technology of SQUIDs and SQUID Systems, WILEYVCH Verlag GmbH \& Co. KGaA, Weinheim (2004). 
[12] C. W. J. Beenakker, Annu. Rev. Conden. Matter Phys. 4, 113 (2013).

[13] P. A. Rosenthal, M. R. Beasley, K. Char, M. S. Colclough, and G. Zaharchuk, Appl. Phys. Lett. 59, 3482 (1991).

[14] I. Sochnikov,A. J. Bestwick,J. R. Williams, . M. Lippman, I. R. Fisher,D. GoldhaberGordon, J. R. Kirtley and K. A. Moler, Nano Lett. 13, 3086 (2013).

[15] M. Veldhorst, M. Snelder, M. Hoek, T. Gang, V. K. Guduru, X. L. Wang, U. Zeitler, W. G. van der Wiel, A. A. Golubov, H. Hilgenkamp, and A. Brinkman, Nat.Mater. 11, 417 (2012).

[16] I. Sochnikov, L. Maier, C. A. Watson, J. R. Kirtley, C. Gould, G. Tkachov, E. M. Hankiewicz, C. Brüne, H. Buhmann, L. W. Molenkamp and K. A. Moler, Phys. Rev. Lett. 114, 066801 (2015).

[17] E. Bocquillon, R. S. Deacon, J. Wiedenmann, P. Leubner, T. M. Klapwijk, C. Brüne, K. Ishibashi, H. Buhmann, and L. W. Molenkamp, http://arxiv.org/abs/1601.08055 (2016).

[18] C. Kurter, A. D. K. Finck ,P. Ghaemi, Y. S. Hor and D. J. Van Harlingen, Nat. Commun. 6, 7130 (2015).

[19] J. R. Williams, A. J. Bestwick, P. Gallagher, Seung Sae Hong, Y. Cui, Andrew S. Bleich, J. G. Analytis, I. R. Fisher and D. Goldhaber-Gordon, Phys. Rev. Lett. 109, 056803 (2012).

[20] L. Fu and C. L. Kane, Phys. Rev. B 79, 161408 (2009).

[21] D.M. Badiane, M. Houzet, and J. S. Meyer, Phys. Rev. Lett. 107, 177002 (2011).

[22] A. C. Potter and L. Fu, Phys. Rev. B 88, 121109 (2013).

[23] M. Snelder, C. G. Molenaar, Y. Pan,D. Wu, Y. K. Huang, A. de Visser, A. A. Golubov, W. G. van der Wiel, H. Hilgenkamp, M. S. Golden and A. Brinkman, Supercond. Sci. Technol. 27, 104001 (2014).

[24] A. I. Gubin, K. S. Il'in, S. A. Vitusevich, M. Siegel, and N. Klein, Phys. Rev. B 72, 064503 (2005).

[25] L. Galletti, S. Charpentier, M. lavarone, P. Lucignano, D. Massarotti, R. Arpaia, Y. Suzuki, K. Kadowaki, T. Bauch, A. Tagliacozzo, F. Tafuri, and F. Lombardi, Phys. Rev. B 89, 134512 (2014).

[26] S. Shapiro, Phys. Rev. Lett. 11, 80 (1963). 
[27] A. Pal, Z. Barber, J. Robinson and M. Blamire, Nat. Commun. 5, 3340 (2014).

[28] P. San-Jose, E. Prada and R. Aguado, Phys. Rev. Lett. 108, 257001 (2012).

[29] A. Kitaev, Physics Uspekhi 44, 131 (2001).

[30] J. Wiedenmann, E. Bocquillon, R. S. Deacon, S. Hartinger, O. Herrmann, T. M. Klapwijk, L. Maier, C. Ames, C. Brüne, C. Gould, A. Oiwa, K. Ishibashi, S. Tarucha, H. Buhmann and L. W. Molenkamp, Nat. Commun. 7, 10303 (2016).

[31] M. Chauvin, P. vom Stein, H. Pothier, P. Joyez, M. E. Huber, D. Esteve, and C. Urbina, Phys. Rev. Lett. 97, 067006 (2006).

[32] P. Dubos, H. Courtois, O. Buisson, and B. Pannetier, Phys. Rev. Lett. 87, 206801 (2001).

[33] J. C Cuevas, J. Heurich, A. Martn-Rodero, A. L. Yeyati and G. Schön, Phys. Rev. Lett. 88, 157001 (2002).

[34] G. Tkachov and E. M. Hankiewicz, Phys. Rev. B 88, 075401 (2013). 


\section{Summary}

In this dissertation, we presented a combined thin film growth and quantum transport study on superconductor topological insulator hybrid devices. Understanding of the electronic properties of topological insulators (Tls), their preparation in high quality thin film form and their interaction with other materials such as s-wave superconductors and ferromagnets, is needed before Tls can be implemented in novel electronic devices. Opportunities arising from understanding the electronic properties of these materials will pave the way to the creation and manipulation of Majorana zero-energy modes, which can serve as a building block for a topological quantum bit, and to practical applications in spintronic devices.

In Chapter 1, we put forward the practical necessity of using TI thin films and their combination with s-wave superconductors in hybrid topological Josephson junctions devices. The exciting proposals to exploit $\mathrm{TI}$ surface states have been hampered by the bulk conductivity associated with the unavoidable presence of defects in $\mathrm{TI}$ materials, e.g $\mathrm{Bi}_{2} \mathrm{Te}_{3}, \mathrm{Bi}_{2} \mathrm{Se}_{3}$ and $\mathrm{Sb}_{2} \mathrm{Te}_{3}$, as well as impurities on their surfaces. Using the molecular beam epitaxy technique, high-quality bulk insulating $\mathrm{TI}$ thin films can be prepared. Thin films also offer the flexibility to control the sample thickness, the possibility of in-situ preparing films and capping them right after growth to avoid extrinsic defects. Hybrid topological insulator Josephson devices are predicted to host $4 \pi$-periodic gapless Andreev bound states (ABS), which are regarded as the hallmark for the detection of a Majorana fermion mode in condensed matter systems. When ultra-thin films in the quantum spin Hall insulator regime are used, the $4 \pi$-periodicity features should appear strongly in topological Josephson junctions fabricated on such ultra-thin films.

In the first part (Chapter 2 and Chapter 3 ) of this dissertation, we identify the conditions under which molecular beam epitaxy gives reproducible results for obtaining high quality, stoichiometric 
$\mathrm{Bi}_{2} \mathrm{Te}_{3}$ thin films; and then carried out a magnetotransport characterisation on $\mathrm{Bi}_{2} \mathrm{Te}_{3}$ samples. The growth mode of $\mathrm{Bi}_{2} \mathrm{Te}_{3} \mathrm{TI}$ thin films, under the optimal molecular beam epitaxy growth conditions, is studied in Chapter 2. The two-step growth procedure is identified to give high quality $\mathrm{Bi}_{2} \mathrm{Te}_{3}$ thin films of terrace-step surface morphology with less defects and large triangular mounds. The characteristic triangular-shaped terraces and steps at the surface of $\mathrm{Bi}_{2} \mathrm{Te}_{3}$ thin films reflect its hexagonal crystal structure inside the (0001) plane. Chapter 3 presents magnetotransport results of a $70 \mathrm{~nm}$ thick $\mathrm{Bi}_{2} \mathrm{Te}_{3}$ thin film. The measured magnetoresistance data establish the topological nature of the transport, such as a non-trivial Berry phase in $\mathrm{Bi}_{2} \mathrm{Te}_{3}$ samples. The nonlinear behaviour in the Hall signal shows the presence of multiple bands or a scattering amplitude that depends on magnetic field. The two-band analysis of the measured $R_{x y}$ did not correctly reproduce the longitudinal resistance behaviour. On the other hand, using a bulk magnetoresistance model it was possible to fit simultaneously the longitudinal and transverse conductivity data. The agreement between the mobility $\mu_{\text {Hall }}$ extracted from Hall data and the mobility $\mu_{S d H}$ estimated from Shubnikov de haas $(\mathrm{SdH})$ oscillations plausibly indicates that bulk states dominate the measured $\mathrm{SdH}$ oscillations. A favourable route to reach high quality bulk insulating $\mathrm{TI}$ thin films is to grow thin films and perform all subsequent characterizations under ultra-high vacuum conditions without exposing samples to ambient conditions.

In the second part (Chapter 4 and Chapter 5) of this dissertation, we succeed to prepare high quality intrinsically insulating $\mathrm{Bi}_{2} \mathrm{Te}_{3} \mathrm{TI}$ thin films, and subsequently demonstrate the importance of capping these films before exposure to ex-situ contaminations. In Chapter 4, we achieve absence of a conducting bulk shunt in $\mathrm{Bi}_{2} \mathrm{Te}_{3}$ thin films without resorting to techniques like counterdoping or using (off-)stoichiometric compounds. Combining in-situ spectroscopy and in-situ topography of $\mathrm{Bi}_{2} \mathrm{Te}_{3}$ thin films, we show that in-situ storage in ultra-high vacuum and exposure to pure oxygen at atmospheric pressures leave the band structure at the surface unaffected. Breaking vacuum and exposing the sample surface to air results in notable changes in the surface band structure and the formation of extrinsic defects at the surface of the sample. Ex-situ contaminations of the surface cause a moderate increase in the doping level of the sample. In Chapter 5 , an effective in-situ protective capping method of $\mathrm{Bi}_{2} \mathrm{Te}_{3} \mathrm{TI}$ films is demonstrated. The $\mathrm{Al}_{2} \mathrm{O}_{3}$ and Te layers are identified as capping overlayers that preserve the topological surface states of $\mathrm{Bi}_{2} \mathrm{Te}_{3}$ thin films. Employing both capping materials, we find a significant decrease of the sample's carrier density. Using the $\mathrm{SrTiO}_{3}$ substrate as a gate dielectric, we tune the carrier density in topological Hall bar devices; and then demonstrate a full depletion of bulk carriers, thus giving access to a transport regime dominated by surface conduction. This ability to access TI surface states is an important step towards device applications in the field of quantum information and spintronics. 
In the third part of this dissertation (Chapter 6 and Chapter 7), we study the Josephson physics in superconductor-topological insulator hybrid devices. In Chapter 6, we successfully realize a combined gate-tunable Josephson supercurrent and normal state transport of Josephson junctions and Hall bar devices fabricated side-by-side on $\mathrm{Bi}_{2} \mathrm{Te}_{3} \mathrm{TI}$ thin films. A comparative study of the gate-dependence of both the magnetotransport data for a Hall bar device and of the supercurrent in Josephson junctions demonstrate strong correlations between the supercurrent transport and the charge distribution in $\mathrm{Bi}_{2} \mathrm{Te}_{3}$ samples. In both the gate voltage dependence of the critical current and of the carrier density, we identify different gate voltage regimes with distinct behaviour for the two electrical field polarities. For negative gate voltage, the supercurrent is independent of the applied electric field, this behaviour is interpreted as a sign that we have effectively decoupled the bottom part of the $\mathrm{TI}$, and the Josephson supercurrent is flowing only in the top portion of the device, thus the critical current stays essentially constant. In the last chapter, Chapter 7, for a thinner $\mathrm{Bi}_{2} \mathrm{Te}_{3}$ sample of $6 \mathrm{~nm}$ thickness in the vicinity of the 2D quantum spin Hall regime, we discuss experimental indications of supercurrent originating from states speculated to be located on the edges of the Josephson junction. The magnetic field dependence of the supercurrent, at different gate voltages, exhibits unusual diffraction patterns that are reminiscent of those in superconducting quantum interference devices (SQUIDs). The observation of anomalies in Shapiro steps of $\mathrm{Nb}-\mathrm{Bi}_{2} \mathrm{Te}_{3}-\mathrm{Nb}$ Josephson junctions at certain gate voltages further corroborate with unusual diffraction patterns, and make it plausible that such anomalies could originate from $1 \mathrm{D}$ edge transport at both edges of the sample. For future experiments, it is imperative to use ultra-thin samples with quantum spin Hall effect behaviour in order to reach the single channel regime, which is rather the ideal experimental configuration to detect Majorana zero-energy modes. This work sets a pathway towards intrinsic topological quantum devices and pave the way for more elaborate experimental investigations on induced superconductivity in three-dimensional Bi-based TIs. 



\section{Samenvatting (Summary in Dutch)}

Dit proefschrift behelst onderzoek naar gecombineerde dunnelaagsfabricage en kwantumtransportmetingen van hybride structuren van supergeleiders en topologische isolatoren. Begrip van de elektronische eigenschappen van gefabriceerde topologische isolatoren, de factoren die de kwaliteit van de dunne laag bepalen en de interactie van topologische isolatoren met andere materialen zoals supergeleiders met s-symmetrie of ferromagneten is noodzakelijk voordat topologische isolatoren kunnen worden geïntegreerd in nieuwe elektronische schakelingen. De mogelijkheden die ontstaan door het begrijpen van de elektronische eigenschappen van deze materialen zijn praktische toepassingen in spintronica en het creeëren en manipuleren van Majorana toestanden als mogelijke bouwsteen voor de topologische kwantumbit.

In Hoofdstuk 1 wordt de praktische noodzaak uiteengezet voor het gebruik van dunnelaags topologische isolatoren en het nut van de combinatie met supergeleiders in hybride topologische Josephson structuren. Uitdagende voorstellen om de oppervlaktetoestanden van topologische isolatoren te benutten, worden belemmerd door de bulkgeleiding die samenhangt met de onontkoombare aanwezigheid van defecten in topologische isolatoren zoals $\mathrm{Bi}_{2} \mathrm{Te}_{3}, \mathrm{Bi}_{2} \mathrm{Se}_{3}$ en $\mathrm{Sb}_{2} \mathrm{Te}_{3}$, alsmede oneffenheden op hun oppervlakken. Door middel van het opbrengen van epitaxiale lagen met moleculaire bundels kunnen dunnelaags topologische isolatoren worden gemaakt van hoge kwaliteit en zonder bulkgeleiding. Daarnaast biedt dunnelaagsfabricage de mogelijkheid om de dikte van de topologische isolator op de toepassing af te stemmen en om topologische lagen in-situ direct na de groei af te dekken om daarmee extrinsieke defecten te voorkomen. Het is voorspeld dat topologische Josephson contacten $4 \pi$-periodieke Andreev toestanden kunnen bevatten, welke worden gezien als de manifestatie van het Majorana fermion in vastestofsystemen. Wanneer ultradunne topologische isolatoren in het kwamtum spin Hall isolator regime worden gebruikt, zou 
de $4 \pi$-periodiciteit duidelijk moeten verschijnen in topologische Josephson contacten.

In het eerste deel van dit proefschrift (Hoofdstuk 2 en Hoofdstuk 3) stellen we de condities vast waaronder de fabricage met moleculaire bundels reproduceerbare resultaten geeft voor het verkrijgen van hoge kwaliteit, stoïchiometrische lagen van $\mathrm{Bi}_{2} \mathrm{Te}_{3}$. Vervolgens zijn de magnetotransporteigenschappen van deze $\mathrm{Bi}_{2} \mathrm{Te}_{3}$ monsters gekarakteriseerd. De groeimodus van $\mathrm{Bi}_{2} \mathrm{Te}_{3}$ dunne lagen onder de ideale groeicondities wordt bestudeerd in Hoofdstuk 2. Er kan worden geconcludeerd dat het tweetraps groeiproces leidt tot hogere kwaliteit $\mathrm{Bi}_{2} \mathrm{Te}_{3}$ dunne lagen met een gestapte oppervlaktemorfologie, minder afwijkingen en grote, driehoekige pyramide-achtige structuren. De karakteristieke driehoekige terrassen op het oppervlak van $\mathrm{Bi}_{2} \mathrm{Te}_{3}$ dunne lagen tonen de hexagonale kristalstructuur in het (0001) vlak. Hoofdstuk 3 presenteert de magnetotransportresultaten van een $70 \mathrm{~nm}$ dikke $\mathrm{Bi}_{2} \mathrm{Te}_{3}$ laag. De gemeten magnetoweerstand bevestigt de topologische origine van het transport, zoals bijvoorbeeld blijkt uit de niet-triviale Berry fase. Het niet-lineaire gedrag van het Hall signaal toont de aanwezigheid van meerdere banden of van een verstrooiingsamplitude die van het magnetisch veld afhangt. Een normale twee-band analyse van de gemeten $R_{x y}$ is niet in staat om de lineaire longitudinale weerstand te reproduceren. Daar staat tegenover dat het met een topologisch model voor de bulkgeleiding wel mogelijk is om tegelijkertijd de longitudinale en transversale geleidingsdata te verklaren. De overeenkomst tussen de mobiliteit $\mu_{H a l l}$, verkregen uit de Hall data, en de mobiliteit $\mu_{S d H}$, geschat uit Shubnikov - de Haas oscillaties, geeft aan dat het plausibel is dat de bulktoestanden domineren in de gemeten Shubnikov - de Haas oscillaties. Een gunstige route voor het realiseren van dunnelaags topologische isolatoren zonder bulkgeleiding is het doen van de fabricage van de lagen en alle daaropvolgende karakterisatie in een ultrahoog vacuüm zonder de monsters bloot te stellen aan de omgeving.

In het tweede deel (Hoofdstuk 4 en Hoofdstuk 5) van dit proefschrift zijn we erin geslaagd om intrinsiek bulk-isolerende $\mathrm{Bi}_{2} \mathrm{Te}_{3}$ topologische isolator lagen te maken en vervolgens het belang aan te tonen van het afdekken van deze films alvorens ze bloot te stellen aan ex-situ verontreinigingen. In hoofdstuk 4 is de afwezigheid van bulkgeleiding bereikt zonder terug te vallen op technieken zoals tegendotering en het gebruik van (niet-)stoichiometrische samenstellingen. In-situ spectroscopie en in-situ topografie van $\mathrm{Bi}_{2} \mathrm{Te}_{3}$ lagen combinerend, laten we zien dat het insitu opslaan in ultrahoog vacuüm en het blootstellen aan pure zuurstof bij atmosferische drukken geen effect hebben op de bandenstructuur aan het oppervlak. Het breken van het vacuüm en het blootstellen van het monsteroppervlak aan lucht resulteert in duidelijke veranderingen in de oppervlaktebandenstructuur en de formatie van extrinsieke defecten aan het oppervlak van het monster. Ex-situ verontreinigingen van het oppervlak veroorzaken een verhoging van het doteringsniveau van het monster. In Hoofdstuk 5 wordt een effectieve in-situ afdekkingsmethode 
ter bescherming van $\mathrm{Bi}_{2} \mathrm{Te}_{3}$ films ontwikkeld. $\mathrm{Al}_{2} \mathrm{O}_{3}$ en Te dunne lagen zijn geïdentificeerd als geschikte afdekkingslagen waarmee de topologische oppervlaktetoestanden van $\mathrm{Bi}_{2} \mathrm{Te}_{3}$ lagen behouden blijven. Door afdekkingsmaterialen in te zetten, vinden we een significante afname van de ladingsdragerdichtheid van het monster. Het $\mathrm{SrTiO}_{3}$ substraat als diëlectricum gebrui-kend, variëren we de ladingsdichtheid in topologische Hall structuren en demonstreren dan een volledige depletie van de bulk ladingsdragers. Dit geeft toegang tot een transportdomein dat gedomineerd wordt door oppervlaktegeleiding, hetgeen een belangrijke stap is richting toepassingen in het veld van de kwantuminformatica en spintronica.

In het derde deel van dit proefschrift (Hoofdstuk 6 en Hoofdstuk 7) bestuderen we de Josephson fysica in supergeleider-topologische isolator hybride structuren. In Hoofdstuk 6, realiseren we een instelbare $\mathrm{Bi}_{2} \mathrm{Te}_{3}$ Josephson superstroom en een Hall structuur waarmee gelijktijdig het transport in de normale toestand van $\mathrm{Bi}_{2} \mathrm{Te}_{3}$ gemeten kan worden. Een vergelijkend onderzoek naar de afhankelijkheid van de extern aangelegde spanning over het diëlectricum van zowel de magnetotransportdata als het superstroomtransport demonstreert een sterke samenhang tussen het superstroomtransport en de ladingsverdeling in de $\mathrm{Bi}_{2} \mathrm{Te}_{3}$ monsters. In de poortspanningsafhankelijkheid van zowel de kritische stroom als de ladingsdragersdichtheid zien we verschillend gedrag voor de twee polariteiten van het extern aangelegde elektrisch veld. Bij negatieve poortspanningen is de superstroom onafhankelijk van het aangeboden veld. Dit gedrag wordt geïnterpreteerd als het effectief loskoppelen van het onderste deel van de topologische isolator zodat de Josephson superstroom slechts in het bovenste deel van de structuur vloeit, waardoor de kritische stroom in feite constant blijft. In het laatste hoofdstuk, Hoofdstuk 7, bediscussiëren we de experimentele aanwijzingen voor een superstroom die zijn oorsprong heeft in toestanden die zich mogelijkerwijze aan de rand van de structuur bevinden. Hiervoor beschouwen we een dunner $\mathrm{Bi}_{2} \mathrm{Te}_{3}$ monster, met een dikte van $6 \mathrm{~nm}$, in de buurt van het 2D kwantum spin Hall gebied. De afhankelijkheid van het magneetveld van de superstroom bij verschillende poortvoltages toont ongebruikelijke diffractiepatronen die doen denken aan die van supergeleidende kwantuminterferentie. De observatie van ongebruikelijkheden in de Shapiro-stappen van $\mathrm{Nb}-\mathrm{Bi}_{2} \mathrm{Te}_{3}-\mathrm{Nb}$ Josephson contacten bij bepaalde poortspanningen zetten deze ongewone diffractiepatronen kracht bij en maken het plausibel dat dergelijke anomaliën hun oorsprong kunnen hebben in 1D transport op beide randen van het monster. Bij toekomstige experimenten is het van belang dat ultradunne monsters met het kwantum spin Hall effect gebruikt worden, zodateen regime wordt bereikt met slechts een loodrechte Majorana toestand. Dit werk toont een pad richting intrinsiek topologische kwantumstructuren en maakt de weg vrij voor uitgebreidere, experimentele onderzoeken naar geïnduceerde supergeleiding in topologische isolatoren gebaseerd op Bi. 



\section{Acknowledgements}

A Ph.D. program is a long journey, and I wouldn't have been able to make it through without the scientific, technical and moral assistance of many. At the end of my Ph.D., I would like to gratefully acknowledge and thank all the people who contributed to the success of my Ph.D. during the past 4 years.

First, I would like to thank my promotors, Prof. Alexander Brinkman and Prof. Hans Hilgenkamp for giving me the great opportunity to join their groups, interfaces and correlated electron systems (ICE) and quantum transport in matter groups (QTM), as a Ph.D. candidate. You introduced me to the fascinating world of quantum materials, particularly to the novel topological insulator materials, their growth in thin film form and structuring into quantum devices. Your guidance, insight and support at different stages of my Ph.D. were invaluable and motivated me to keep moving forward. Thank you also for giving me sufficient freedom in my research and supporting me to attend different scientific meetings within and outside my research field, especially those on the development of science in Africa. It was a good opportunity to make professional connections, which will be beneficial for my future professional career and beyond. Furthermore, coming from a very different cultural background, the support and encouragement that I received from you at the early stage of my Ph.D. did help me to adapt well to a new scientific and social environment.

The excellent working atmosphere in the ICE-QTM groups, the friendly $\mathrm{PhD}$ colleagues and Postdoc researchers, the efficient administrative and technical staff made the work (described) in this dissertation possible. You all helped me to have an enjoyable time in the lab. I shall never forget the good times we had as a group in going ice skating, which was my very first time in an ice rink; and on different sailing trips ( even on rainy days!). Many thanks to Ans Veenstra and Inke in de Wal for your outstanding help with all the formalities. In particular, I would like to 
thank my colleagues Denise Leusink, Marieke Snelder and Martin Stehno, with whom I shared an office with during my Ph.D. time. I enjoyed working and discussing with you. My special thanks to you Martin Stehno for your help in electronic transport measurements of my samples using the Triton. I learned a lot from you, about doing "proper" electronic transport measurements, as you used to tell me. Thank you also for taking time to proof-read most parts of my dissertation. My sincere thanks to Femi Ojambati and Joris Voerman for being my paranymphs.

Regarding collaborations within and outside the university, first and foremost $\mathrm{i}$ would like to thank Prof. Gertjan Koster and colleague Ph.D. candidates and Postdocs in the inorganic material science (IMS) group for the excellent collaborative atmosphere. I enjoyed working with you at the COMAT system for the growth and preliminary characterization of the $\mathrm{Bi}_{2} \mathrm{Te}_{3}$ thin films (described in this dissertation). Thanks also to Prof. Harold Zandvliet and his research group (in particular, René Heimbuch) for collaboration related to scanning tunnelling microscopy and spectroscopy (STM and STS) measurements on $\mathrm{Bi}_{2} \mathrm{Te}_{3}$ thin films. My special thanks to Prof. Mark Golden, Erik van Heumen and your research groups at the University of Amsterdam. It was nice working with you on angle resolved photoemission spectroscopy (ARPES) measurements on my $\mathrm{Bi}_{2} \mathrm{Te}_{3}$ thin film samples transported to Amsterdam using the UHV suitcase. I would also like to thank Prof. Hiroaki Myoren from Saitama University in Japan. It was nice working with you in the fabrication steps of the Josephson junctions on $\mathrm{Bi}_{2} \mathrm{Te}_{3}$ thin films during your sabbatical leave at the University of Twente.

Let me also acknowledge support I received from the technical personnel during my Ph.D. program. I thank Dominic Post, Frank Roesthuis and Dick Veldhuis for your technical support during the upgrading stage of the growth chamber and, most importantly, when I was having difficulties with the most broken equipment (MBE), the evaporation chamber, that I was using for topological insulator thin film growth. I acknowledge the assistance of Laura Vargas in maintaining and keeping running the XPS system at the COMAT.

I am also thankful to different institutions that supported my Ph.D. research financially and/or played a role in the development of my future career during the course of my Ph.D. program. I thank FOM for both supporting my Ph.D financially and giving me the opportunity to work as junior scientist at the University of Twente. I am very thankful to the personnel of FOM for arranging all the work related permits in the Netherlands; in particular to you Maria Teuwissen for all your help regarding the Dutch VISA formalities for me and my family. I also thank both the African Institute for Mathematical Sciences (AIMS) and the Robert Bosch Stiftung for providing me the opportunity to attend the interdisciplinary $65^{\text {th }}$ Lindau Nobel laureate meeting. It was a memorable and inspiring opportunity of getting in contact with different Nobel Laureates from different disciplines; and to learn from them. Also, it was a favourable moment to meet and 
discuss with fellow young scientists of different nationalities. These connections will be valuable for the development of my future career after this $\mathrm{Ph} . \mathrm{D}$ program.

Lastly and most importantly, I would like to thank my family. Special thanks to my beloved wife, Claudine M. Uwibambe and my daughter Ineza P. Ngabonziza for their encouragement, unceasing moral assistance and for supporting me daily during the last 4-years. Your emotional support strengthened and re-energized me during the challenging times of the Ph.D. journey. Thanks to my lovely parents, brother and sisters for your unconditional assistance at every stage of my life and your support in this Ph.D. program and my overall education journey. 